\title{
Investigating post-translational modifications and novel interaction partners of otoferlin
}

\author{
Dissertation \\ For the award of the degree \\ “Doctor of Philosophy" (Ph.D.) \\ Division of Mathematics and Natural Sciences \\ of the Georg-August-Universität Göttingen \\ within the doctoral program "Sensory and Motor Neuroscience" \\ of the Georg-August University School of Science (GAUSS)
}

submitted by

Andreia Filipa Pereira Cepeda

from

Lisbon, Portugal

Göttingen, September 2019 



\section{Members of the Thesis Advisory Committee}

PD Dr. Ellen Reisinger

(Supervisor and First Reviewer)

Prof. Dr. Silvio O. Rizzoli

(Second Reviewer)

Prof. Dr. Manuela Schmidt
Molecular Biology of Cochlear Neurotransmission Group,

Department of Otorhinolaryngology and InnerEarLab,

University Medical Center Göttingen

Göttingen, Germany

Department of Neuro- and Sensory Physiology,

University Medical Center Göttingen

Göttingen, Germany

Somatosensory Signaling and Systems Biology Group, Max Planck Institute of Experimental Medicine

Göttingen, Germany

\section{Further Members of the Examination Board}

Prof. Dr. Henning Urlaub

PD Dr. Sven Thoms

Prof. Dr. Ralf Heinrich
Bioanalytical Mass Spectrometry Group,

Max Planck Institute for Biophysical Chemistry,

Göttingen, Germany

Department of Neuropaediatrics,

University Medical Center Göttingen

Göttingen, Germany

Department of Cellular Neurobiology,

Schwann-Schleiden Research Centre,

Göttingen, Germany

Date of the oral examination: 21 October 2019 



\section{Affidavit}

I hereby declare that this dissertation entitled "Investigating post-translational modifications and novel interaction partners of otoferlin" was written independently and with no other sources or aids than quoted.

Andreia Cepeda

Göttingen, 9 September 2019 

"Valeu a pena? Tudo vale a pena

Se a alma não é pequena. Quem quer passar além do Bojador Tem que passar além da dor.” Fernando Pessoa, in Mensagem 



\section{Contents}

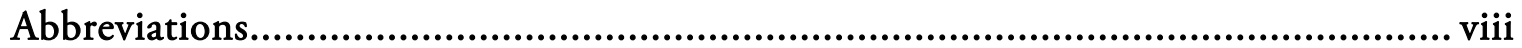

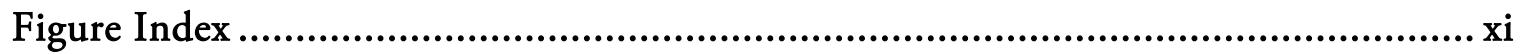

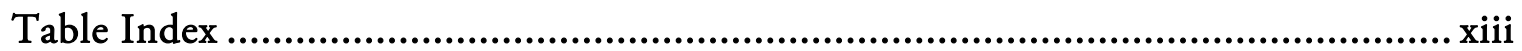

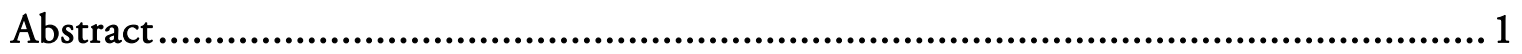

Chapter 1: General Introduction ..........................................................................

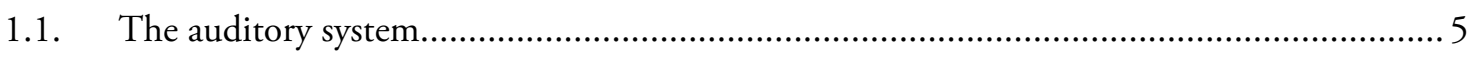

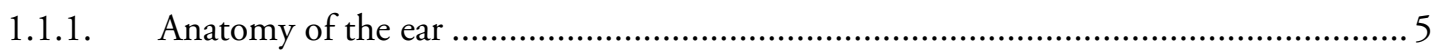

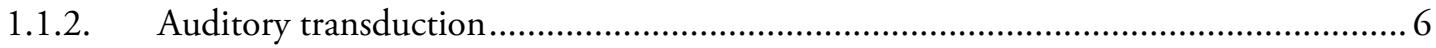

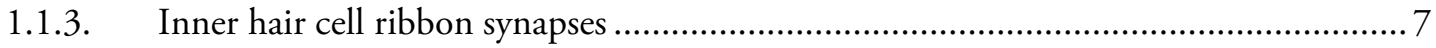

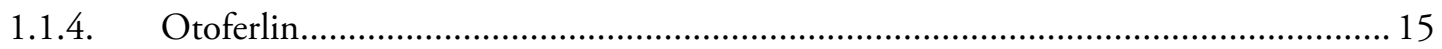

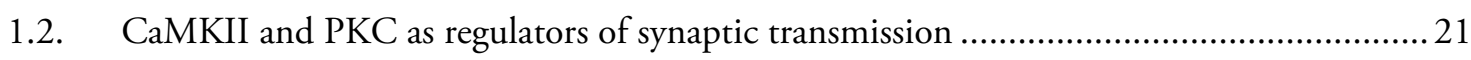

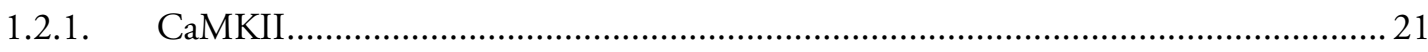

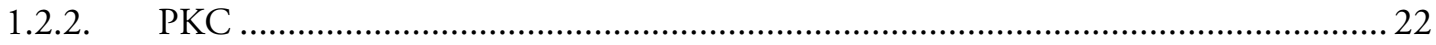

1.2.3. CaMKII and PKC at inner hair cell ribbon synapses............................................... 24

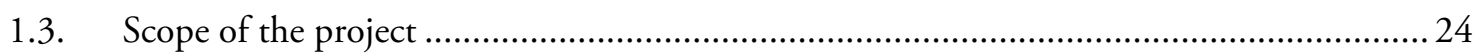

Chapter 2: CaMKII $\delta$ is expressed in the organ of Corti and regulates otoferlin's activity during strong inner hair cell stimulation ........................................................ 27

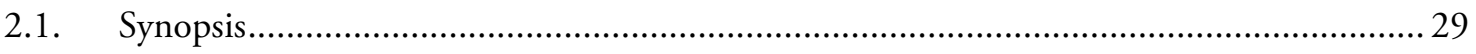

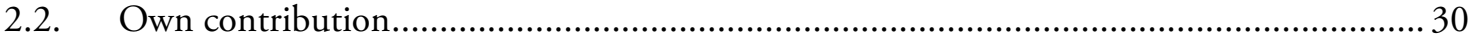

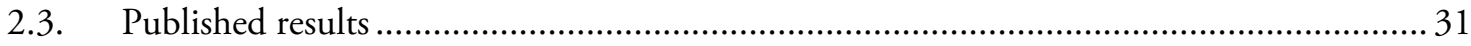

2.3.1. Expression and cellular distribution of different CaMKIIs in the organ of Corti .......31

2.3.2. CaMKII $\delta$ is the predominant CaMKII in rodent IHCs ............................................ 32

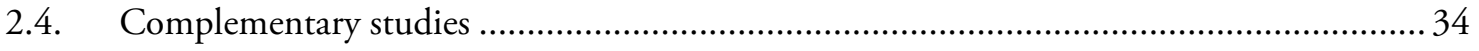

2.4.1. CaMKII interacts with otoferlin in murine IHCs.................................................... 34

2.4.2. Activity-dependent phosphorylation of otoferlin or otoferlin interaction partners in murine IHCs 
Chapter 3: PKC $\alpha$ promotes the interaction of otoferlin with calbindin and regulates endocytosis at auditory inner hair cell synapses.................................................37

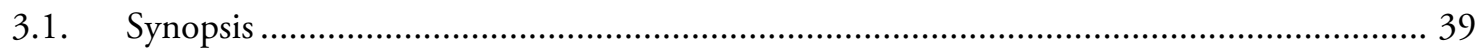

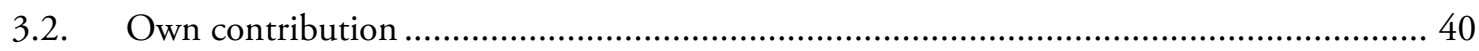

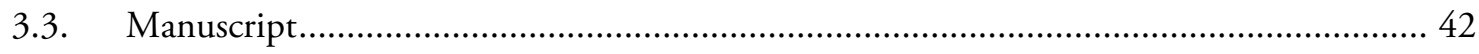

3.3.1. Synopsis and Graphical Abstract........................................................................... 43

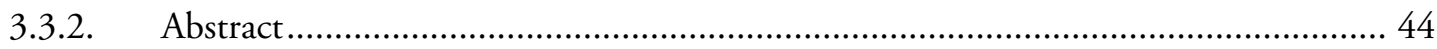

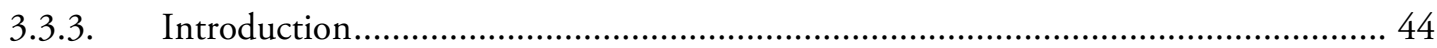

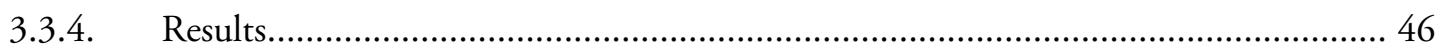

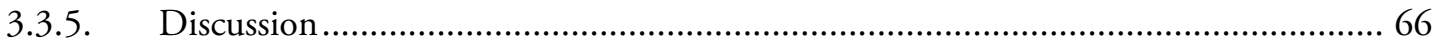

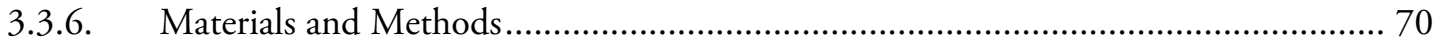

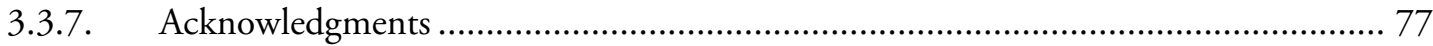

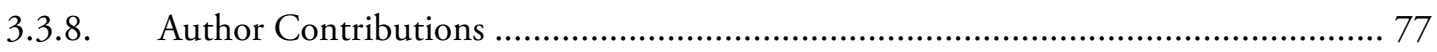

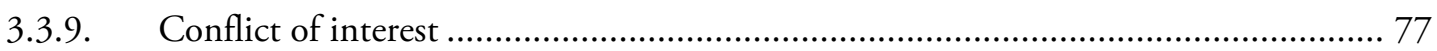

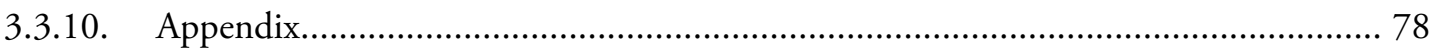

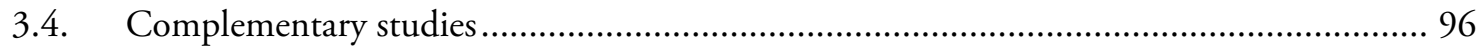

3.4.1. Exploring the possible interaction of otoferlin with other calcium buffer proteins.... 96

Chapter 4: General Discussion........................................................................99

4.1. Activity-dependent regulation of the synaptic vesicle cycle ............................................ 102

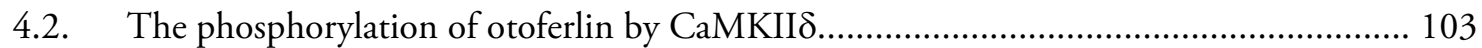

4.2.1. Otoferlin is phosphorylated by CaMKII $\delta$ in an activity-dependent manner............ 104

4.2.2. Phosphorylation by CaMKII $\delta$ affects $\mathrm{Ca}^{2+}$ affinity of otoferlin's $\mathrm{C}_{2}$ domains .......... 105

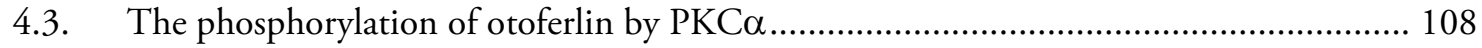

4.3.1. PKC $\alpha$ is expressed in IHCs and redistributes upon activation ............................... 108

4.3.2. PKC $\alpha$ interacts with and phosphorylates otoferlin ................................................ 109

4.3.3. Activity-dependent phosphorylation of otoferlin and/or otoferlin-bearing complexes

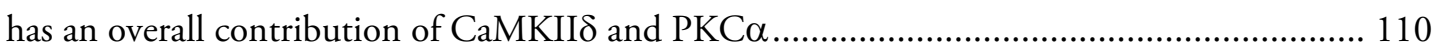

4.3.4. Phosphorylation of otoferlin by PKC $\alpha$ promotes the interaction of otoferlin with

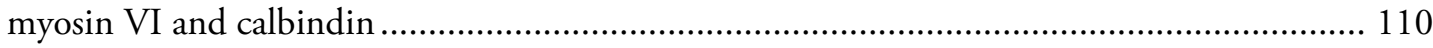

4.3.5. PKC $\alpha$ 's probable role in otoferlin-dependent SV reformation events...................... 111

4.3.6. The otoferlin-calbindin interaction might be important for ultrafast endocytosis... 113

4.3.7. Parvalbumin and calretinin might also interact with otoferlin to mediate $\mathrm{Ca}^{2+}$ dependent exocytic and endocytic events. 
4.4. Potential impact of $O T O F$ mutations in otoferlin's regulation by protein kinases

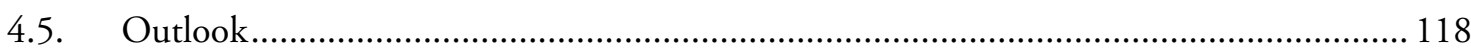

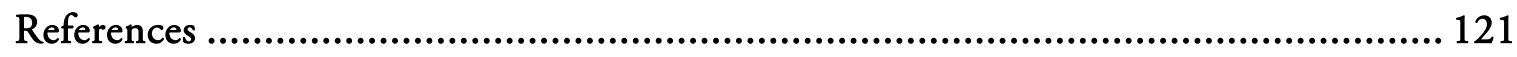

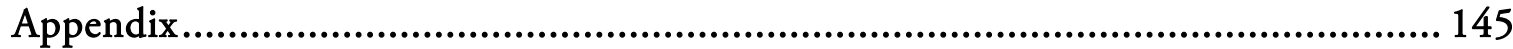

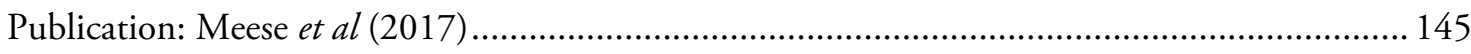

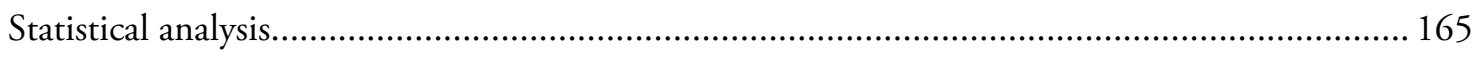

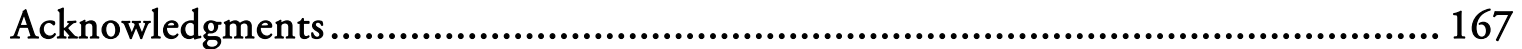

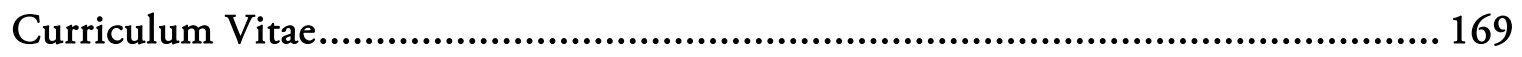




\section{Abbreviations}

$\left[\mathrm{Ca}^{2+}\right]_{e} \quad$ extracellular $\mathrm{Ca}^{2+}$ concentration

$\Delta \mathrm{C}_{\mathrm{m}} \quad$ membrane capacitance changes

AMPA $\quad \alpha$-amino-3-hydroxy-5-methyl-4-isoxazolepropionic

AP-2 adaptor-protein complex 2

AZ active zone

BIM I bisindolylmaleimide I (PKC inhibitor)

$\mathrm{Ca}^{2+} \quad$ calcium ion

CaBP calcium-binding protein

Calb calbindin-D28k (also calbindin)

cDNA complementary DNA

CaM calmodulin

CaMKII $\quad \mathrm{Ca}^{2+} /$ calmodulin-dependent serine/threonine kinase II

CaMKII $\quad \mathrm{Ca}^{2+} /$ calmodulin-dependent serine/threonine kinase II delta

Caml calcium-modulating cyclophilin ligand

CAPS $\quad \mathrm{Ca}^{2+}$-dependent activator protein for secretion

CAST cytomatrix at the active zone-associated structural protein (also ELKS or ERC)

Cav1.3 Cav1.3 L-type voltage-gated calcium channel

CIE clathrin-independent endocytosis

$\mathrm{C}_{\mathrm{m}} \quad$ membrane capacitance

CME clathrin-mediated endocytosis

CNS central nervous system

Co-IP co-immunoprecipitation

$\mathrm{CtBP} 2 \quad$ C-terminal binding protein 2

DAG diacylglycerol

DFNB9 autosomal recessive non-syndromic deafness 9 (OTOF-related deafness)

E embryonic day

ELVs endosome-like vacuoles

EM electron microscopy

ER endoplasmic reticulum 


\begin{tabular}{|c|c|}
\hline F-actin & filamentous actin \\
\hline Fer & ferlin-specific motif \\
\hline FR & functionally redundant \\
\hline $\mathrm{HC}$ & hair cell \\
\hline IHC & inner hair cell \\
\hline IP & immunoprecipitation \\
\hline $\mathrm{K}^{+}$ & potassium ion \\
\hline $\mathrm{KN}-93$ & CaMKII inhibitor \\
\hline LSD & lithium dodecylsulphate \\
\hline MET & mechanoelectrical transduction \\
\hline MP-SV pool & membrane-proximal synaptic vesicle pool \\
\hline MST & microscale thermophoresis \\
\hline Munc13-1 & mammalian unc-13 homologue \\
\hline Munc18-1 & mammalian unc-18 homologue \\
\hline MyoVI & myosin VI \\
\hline NSF & N-ethylmaleimide-sensitive factor \\
\hline OC & organ of Corti \\
\hline $\mathrm{OHC}$ & outer hair cell \\
\hline Otof $f^{-/}$ & otoferlin knock-out mutant mouse model \\
\hline Otof ${ }^{\text {ISISTIISIST }}$ & p.Ile 515 Thr otoferlin knock-in mutant mouse model \\
\hline Otof Pgal/Pa & p.Asp1767Gly (Pachanga) otoferlin mutant mouse model \\
\hline Otof & otoferlin \\
\hline OTOF & otoferlin-encoding gene \\
\hline $\mathrm{P}$ & postnatal day \\
\hline $\mathrm{P} 2 \mathrm{~A}$ & self-cleaving P2A peptide \\
\hline PBS & phosphate buffered saline \\
\hline PCR & polymerase chain reaction \\
\hline $\mathrm{PI}(4,5) \mathrm{P}_{2}$ & phosphatidylinositol-4,5-bisphosphate (also $\mathrm{PIP}_{2}$ ) \\
\hline PKA & cAMP-dependent protein kinase A \\
\hline PKC & protein kinase $\mathrm{C}$ \\
\hline PKN & PKC-related kinase \\
\hline PLA & proximity ligation assay \\
\hline
\end{tabular}


$\mathrm{x} \mid$

PMA phorbol 12-myristate 13-acetate (PKC activator)

PS phosphatidylserine

PSD postsynaptic density

P-Ser phosphoserine (also P-Serine)

$\mathrm{R} \quad$ synaptic ribbon

Rab Ras-related protein

RA-SV pool ribbon-associated synaptic vesicle pool

RIM Rab3-interacting molecule

RIM-BP RIM binding protein

RRP readily releasable pool

s. e. m. standard error of the mean

SDS-PAGE sodium dodecylsulphate polyacrylamide gel electrophoresis

SGNs spiral ganglion neurons

SNAP-25 synaptosomal-associated protein 25

SNARE soluble N-ethylmaleimide-sensitive factor attachment protein receptor

SPL $\quad$ sound pressure level

SRP slowly releasable pool

SV synaptic vesicle

Syt synaptotagmin

TA tail-anchored

TKO triple knock-out

TM transmembrane domain

VAMP vesicle associated membrane protein (also named synaptobrevin)

Vglut vesicular glutamate transporter

WRB tryptophan-rich basic protein

WT wild-type 


\section{Figure Index}

\section{Chapter 1: General Introduction}

Figure 1.1. Structure of the mammalian ear. .5

Figure 1.2. The ribbon synapse, a specialized synapse between inner hair cells (IHCs) and spiral

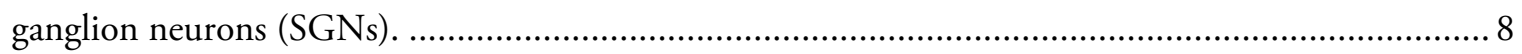

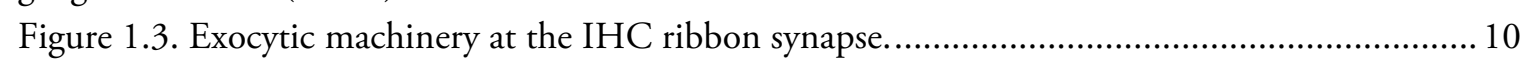
Figure 1.4. Synaptic architecture, synaptic vesicle pools and mechanisms of synaptic vesicle recycling

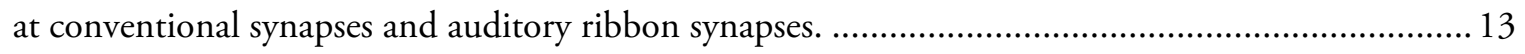

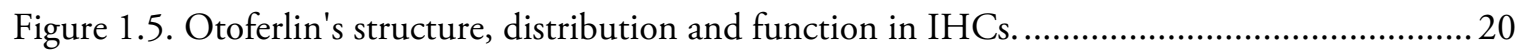

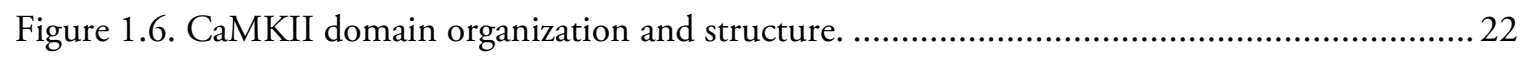

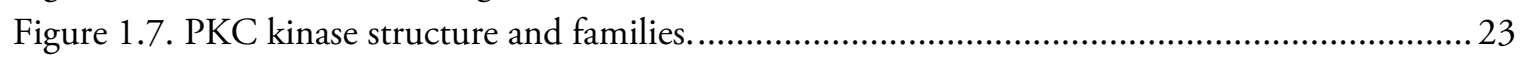

Chapter 2: CaMKIII is expressed in the organ of Corti and regulates otoferlin's activity during strong inner hair cell stimulation

Figure 2.1. Localization of the different CaMKIIs in the murine organ of Corti .............................. 32

Figure 2.2. CaMKII $\delta$ transcripts are predominantly expressed in murine IHCs. ................................ 33

Figure 2.3. Activity-dependent interaction of otoferlin and CaMKII in mouse IHCs......................... 34

Figure 2.4. Phosphorylation of otoferlin complexes is strongly promoted by strong hair cell

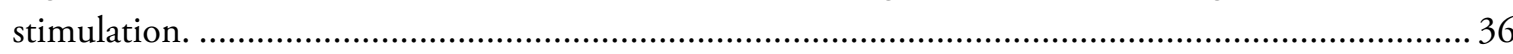

Chapter 3: PKC $\alpha$ promotes the interaction of otoferlin with calbindin and regulates endocytosis at auditory inner hair cell synapses

Figure 3.1. PKC $\alpha$ redistributes to base of IHCs upon strong stimulation and PKC $\alpha$ distribution correlates with otoferlin localization

Figure 3.2. PKC $\alpha$ redistributes to the base of IHCs after strong stimulation, where it is found near

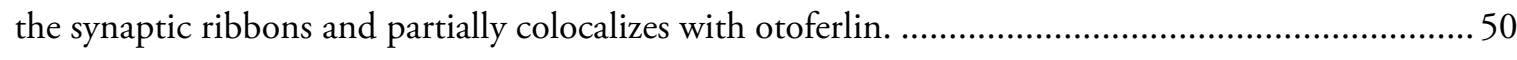

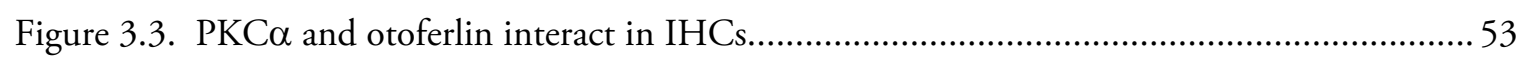

Figure 3.4. PKC $\alpha$ subcellular distribution is affected in otoferlin knock-out IHCs. ...........................55

Figure 3.5. Otoferlin phosphorylation is strongly promoted by hair cell stimulation and can be blocked by combined inhibition of PKC and CaMKII.

Figure 3.6. Otoferlin interacts with myosin VI, but not with Vglut3, in a PKC $\alpha$-dependent manner.

Figure 3.7. The interaction of otoferlin with calbindin is strongly dependent on PKC $\alpha$. ..................61

Figure 3.8. Proximity ligation assays for otoferlin and other calcium buffer proteins........................ 96

Figure 3.9. Parvalbumin immunofluorescence levels in different otoferlin mutant mouse lines. .......97

Figure 3.10. Calretinin immunofluorescence levels in different otoferlin mutant mouse lines. .........98 
Figure EV1. PKC $\alpha$ is expressed in the organ of Corti, in IHCs and OHCs.

Figure EV2. PKC $\alpha$ redistributes upon strong stimulation and treatment with PMA.

Figure EV3. Calbindin and otoferlin immunofluorescence in different otoferlin mutants and in dual-

AAV-transduced Otof $^{-1-}$ and WT IHCs.

Figure EV4. Weak PLA signal between PKC $\alpha$ and calbindin points toward an indirect interaction of the two proteins via scaffolding proteins.

Appendix Figure S1. Validation of the proximity ligation assay in mice organs of Corti. ................. 78

Appendix Figure S2. Negative controls for the proximity ligation assays......................................... 79

Appendix Figure S3. MS/MS spectrum of $\mathrm{m} / \mathrm{z} 632.629^{3+}$ at $38.63 \mathrm{~min}$,

DSQETDGLLPGSRP ${ }^{158}$ pSTR (otoferlin variant 1, NP_001093865.1) ......................................... 80

Appendix Figure S4. MS/MS spectrum of $m / z 449.209^{3+}$ at $42.93 \mathrm{~min}, \mathrm{FL}^{790} \mathrm{pSLSDKDQGR}$

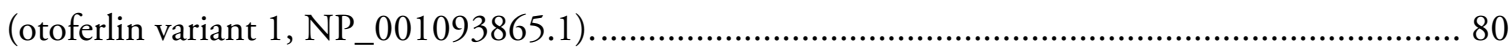

Appendix Figure S5. MS/MS spectrum of $m / z 485.245^{3+}$ at $32.04 \mathrm{~min}, \mathrm{GVQS}^{1184} \mathrm{pSLIHNYKK}$

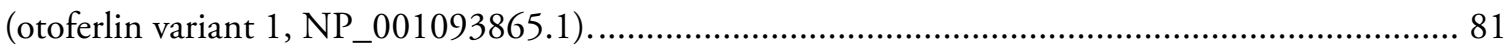

Appendix Figure S6. MS/MS spectrum of $m / z 542.614^{3+}$ at $38.08 \mathrm{~min}$, YTLVGSHAVS ${ }^{1239}$ pSLRR

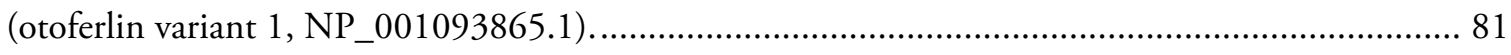

Appendix Figure S7. MS/MS spectrum of $m / z 591.280^{2+}$ at $37.87 \mathrm{~min}, \mathrm{FKG}^{1451} \mathrm{pSLCVYK}$ (otoferlin

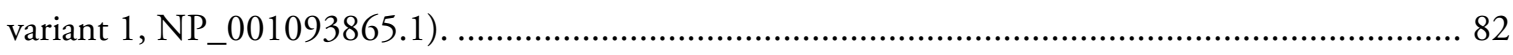

Appendix Figure S8. Total Ion Chromatograms (TICs) of otoferlin in-gel tryptic digests analyzed by

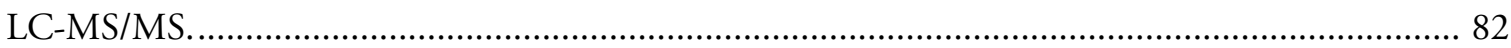

Appendix Figure S9. Extracted Ion Chromatograms (XICs) of otoferlin-derived phosphopeptides. . 83 Appendix Figure S10. Sequence alignment of phosphorylated sites in otoferlin variants 1

(NP_001093865.1) and 4 (NP_001300696.1).

Appendix Figure S11. Sequence alignment of phosphorylated sites in otoferlin from different species.

Appendix Figure S12. PKC is predicted to phosphorylate otoferlin. ............................................. 86

\section{Chapter 4: General Discussion}

Figure 4.1. Position of known OTOF mutations relative to CaMKII $\delta$ phosphorylation sites.

Figure 4.2. Position of p.Glu766del and p.Arg794His mutations relative to PKC $\alpha$ phosphorylation site in FerA domain of otoferlin. 


\section{Table Index}

\section{Chapter 1: General Introduction}

Table 1.1. Main molecular differences between conventional synapses of the mammalian CNS and the IHC ribbon synapse

Chapter 3: PKC $\alpha$ promotes the interaction of otoferlin with calbindin and regulates endocytosis at auditory inner hair cell synapses

Appendix Table S1. Mean averages, sample size and statistical analysis. 87

Appendix Table S2. Prediction of PKC phosphorylation sites in otoferlin.

\section{Appendix}

Appendix Supplementary Table 1. Mean averages, sample size and statistical analysis 



\section{Abstract}

Otoferlin is a large multi- $\mathrm{C}_{2}$-domain protein essential for hearing and fast $\mathrm{Ca}^{2+}$-triggered transmitter release from auditory IHCs. Mutations in the $O T O F$ gene are linked to a form of autosomal recessive non-syndromic hearing loss, DFNB9. Otoferlin is involved in several steps of the synaptic vesicle cycle in IHCs including vesicle fusion, vesicle reformation, vesicle recycling, endocytosis and coupling of exo- and endocytosis. While some progress has been made in understanding its role in IHC synaptic transmission, mechanisms regulating its function have not been studied to date. Second messenger-activated protein kinases regulate synaptic transmission in conventional synapses via phosphorylation of presynaptic proteins thereby controlling presynaptic plasticity, protein interactions within the release apparatus, endocytosis and trafficking events.

In this thesis, I focused on deciphering the role of protein kinases in IHC synaptic transmission. Together with my collaborators, I showed for the first time that synaptic activity in IHC synapses is also regulated by phosphorylation of presynaptic proteins. By combining immunohistochemistry, in situ proximity ligation assays (PLAs), confocal microscopy, realtime PCR, mutagenesis, microscale thermophoresis (MST), pull-downs, coimmunoprecipitations (co-IPs), in vitro assays and mass spectrometry approaches, we showed that $\mathrm{Ca}^{2+} /$ calmodulin-dependent serine/threonine kinase delta (CaMKII $\delta$ ) and protein kinase $\mathrm{C} \alpha(\mathrm{PKC} \alpha)$ phosphorylate otoferlin and regulate its function in rodent IHCs. CaMKII $\delta$ and $\mathrm{PKC} \alpha$ are expressed throughout the cell and both revealed to be in close proximity to otoferlin upon strong stimulation. Physical association between the two kinases and otoferlin was confirmed via binding assays, and kinase-specific phosphorylation sites were retrieved: CaMKII $\delta$ phosphorylates otoferlin in its $\mathrm{C}_{2}$ domains $\left(\mathrm{C}_{2} \mathrm{C}, \mathrm{C}_{2} \mathrm{D}, \mathrm{C}_{2} \mathrm{de}, \mathrm{C}_{2} \mathrm{~F}\right)$ whereas $\mathrm{PKC} \alpha$ seems to target linker regions and the FerA domain (presumed to be involved in membrane-association events), suggesting a combined but distinct action of CaMKII $\delta$ and PKC $\alpha$. Phosphorylation by CaMKII $\delta$ affects the affinity of otoferlin's $\mathrm{C}_{2} \mathrm{C}$ and $\mathrm{C}_{2} \mathrm{~F}$ domains to $\mathrm{Ca}^{2+}$ under physiological conditions. PKC $\alpha$ is targeted upon activation (either pharmacologically or following high $\mathrm{K}^{+}$stimulation) to the basolateral plasma membrane and to endocytic compartments where it interacts with otoferlin. The previously reported interaction of otoferlin with myosin VI appears to be PKC-dependent. Moreover, otoferlin interacts with the EF-hand protein calbindin-D28k in a PKC-dependent manner, whereas PKC $\alpha$ and calbindin-D28k seem not to interact directly. The association of these three proteins probably happens in a sequential fashion and potentially regulates different modes of membrane internalization and may control the dynamics of the synaptic vesicle cycle in IHCs. The PKC-dependent association of otoferlin with calbindin-D28k is especially potentiated 
2 | Abstract

under strong stimulatory conditions and might play a role in clathrin-independent events like ultrafast endocytosis. This mechanism may constitute a molecular switch between different modes of endocytosis, thus providing the grounds for fast and efficient vesicle recycling, hallmarks of IHC ribbon synapses. 
Chapter 1: General Introduction 



\subsection{The auditory system}

Hearing is a unique sensory feature that provides us with acoustic information about our direct surroundings. The evolution of the auditory system in higher vertebrates allowed for prey and predator detection, and particularly in humans, facilitated communication and social interaction, being one of the hallmarks of our information-centered society.

Different mammalian species perceive different ranges of frequencies and intensities of sound stimuli. The human auditory system can encode sounds over a broad dynamic range of 0 to $120 \mathrm{~dB}$ sound pressure level (SPL), spanning frequencies from $20 \mathrm{~Hz}$ to $20 \mathrm{kHz}$ (reviewed in Kandel et al, 2012). Mice are sensitive to sounds ranging from 1 to $100 \mathrm{kHz}$ (Heffner \& Heffner, 2007) and became the most commonly used mammal model in hearing research due to the advantages in genetic manipulation (reviewed in Ohlemiller et al, 2016).

\subsubsection{Anatomy of the ear}
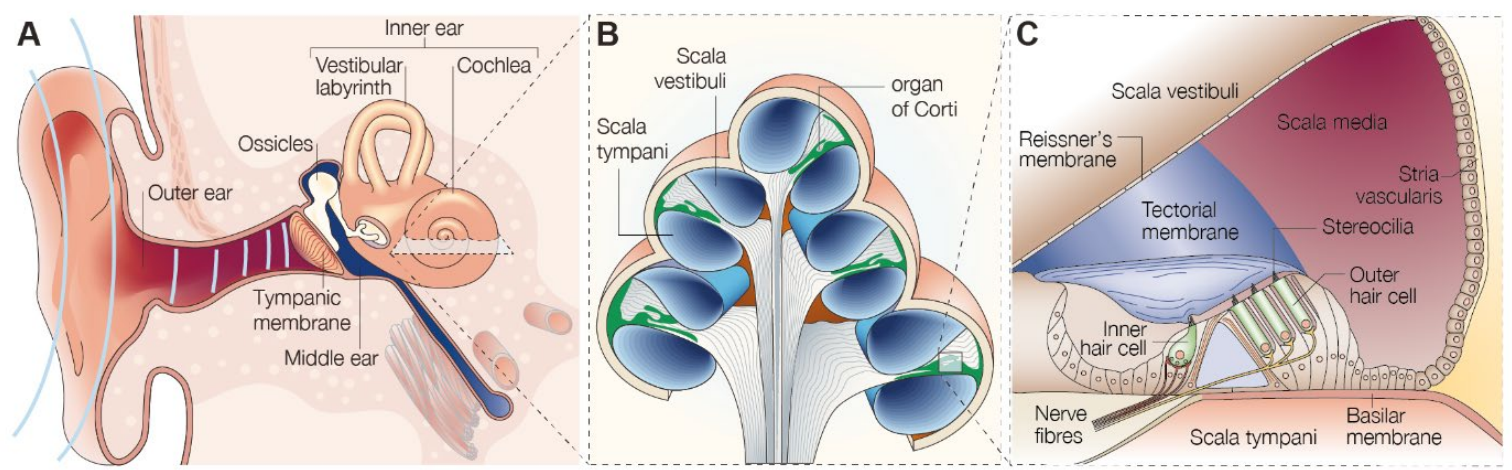

Figure 1.1. Structure of the mammalian ear.

A. Detection of environmental sound begins when incoming sound waves reach the outer ear. Sound propagates through the ossicles in the middle ear, which then transmit it to the cochlea in the inner ear. B. A cross section of the mammalian cochlea, a fluid-filled continuous coiled duct. C. A cross section of one of the cochlear partitions, showing the organ of Corti and the three fluid-filled cochlear chambers, scala tympani, scala media and scala vestibuli. The organ of Corti bears the mechanosensory epithelium composed of one row of inner hair cells and three rows of outer hair cells. Adapted from Frolenkov et al, 2004; Müller \& Barr-Gillespie, 2015.

In the mammalian ear, sound travels through several stations along the auditory pathway in a mechanical-coupling fashion, with sound information being lastly delivered to the cochlea in the inner ear (Figure 1.1A). The cochlea is a snail-shaped structure with a bony core - the modiolus - around which several turns of fluid-filled compartments (scala vestibuli, scala tympani and scala media) are coiled up - two and a half turns in humans (Figure 1.1A-B). Scala vestibuli and scala tympani are filled with perilymph, with a potassium $\left(\mathrm{K}^{+}\right)$concentration of $-5 \mathrm{mM}$, while the scala media is filled with endolymph, with higher $\mathrm{K}^{+}$concentrations of $\sim 160$ 
mM. Reissner's membrane separates scala vestibuli from scala media. Between scala tympani and scala media is the basilar membrane, and on top of it sits the organ of Corti, a specialized sensory epithelium that amplifies and transduces mechanical sound vibrations into an electrical output signal which is then transferred to the brain (see chapter 1.1.2) (Figure 1.1C). In humans the organ of Corti harbors approximately 16000 hair cells (HCs) organized into three rows of outer hair cells (OHCs) and one row of inner hair cells (IHCs). These cells have on their apical surface structures termed stereocilia, mechanically sensitive actin-filled organelles organized in rows of increasing height. The tectorial membrane sits on top of the organ of Corti and forms direct connections to the stereocilia of the OHCs but not of IHCs (Figure 1.1C) (reviewed in Hudspeth, 1997; Kandel et al, 2012).

\subsubsection{Auditory transduction}

The sense of hearing is accomplished by a process known as auditory transduction. The ear converts sound waves in the air into electrical impulses, which are then interpreted by the brain.

As sound enters the ear, it passes through the auditory canal in the outer ear and it reaches the tympanic membrane, which separates the outer from the middle ear. The tympanic membrane then vibrates in response to the sound waves and delivers them to a chain of three bones called the ossicles (malleus, incus and stapes) (Figure 1.1A). Vibrations transmitted by the stapes are drowned into the spiral system through the oval window of the cochlea where they are converted into liquid pressure waves in the fluid-filled space of the cochlea. These are propagated to the apex of the cochlea, ascending through the scala vestibuli, and return to the round window, descending through the scala tympani (Figure 1.1B). Reissner's and basilar membranes are flexible and move in response to the vibrations travelling up the scala vestibuli and down the scala tympani. As the basilar membrane vibrates, OHCs and IHCs, located between the basilar and tectorial membranes, are stimulated by the shearing force between the basilar and tectorial membranes. While OHCs boost the sound stimulus by amplifying the sound-driven basilar membrane vibrations, IHCs convert the sound-induced vibrations into an electrical signal and convey it to the afferent boutons of the spiral ganglion neurons (SGNs). Firstly, the basilar and tectorial membranes' oscillations lead to deflection of the OHCs' stereocilia and to opening of mechanoelectrical transduction (MET) channels in the tips of the stereocilia. $\mathrm{K}^{+}$influx leads to $\mathrm{OHC}$ depolarization and the cell undergoes an oscillation-based alternation of length, amplifying the oscillations which are in turn transferred back to the basilar and tectorial membranes and to the endolymph. Mechanical vibrations in the endolymph are transferred to the IHCs, leading to deflection of their stereocilia and resulting in the opening of MET channels (Fettiplace \& Kim, 2014). The subsequent $\mathrm{K}^{+}$ influx generates a depolarizing receptor potential that scales with sound intensity (Glowatzki $\&$ Fuchs, 2002) (Figure 1.2A). This in turn triggers $\mathbf{C a}^{2+}$ influx via opening of voltage-gated 
calcium $\left(\mathrm{Ca}^{2+}\right)$ channels (Platzer et al, 2000; Brandt et al, 2003) which cluster at the presynaptic release sites, also termed active zones (AZs) (Figure 1.2A), triggering $\mathrm{Ca}^{2+}$-dependent exocytosis and release of neurotransmitter onto the IHC-SGN synaptic cleft (Moser \& Beutner, 2000; Glowatzki \& Fuchs, 2002) (Figure 1.2B). Neurotransmitter release into the synaptic cleft activates AMPA ( $\alpha$-amino-3-hydroxy-5-methyl-4-isoxazolepropionic) receptors at afferent dendrites of the SGNs (Glowatzki \& Fuchs, 2002; Schnee et al, 2011) (Figure 1.2B). The electrical signal is then propagated along the auditory pathway and is processed in the auditory centers of the brain (reviewed in Hudspeth, 1997; Kiang, 2011; Kandel et al, 2012; Fettiplace, 2017).

The entire basilar membrane does not vibrate simultaneously. Instead, specific areas of the basilar membrane move variably in response to different frequencies of sound. This is determined by the width and thickness of the basilar membrane at a particular location. Lower frequencies vibrate the basilar membrane stronger in regions closer to the apex of the cochlea whereas higher frequencies produce vibrations with higher amplitudes closer to the base. This arrangement is known as tonotopic organization (reviewed in Hudspeth, 1997; Frolenkov et al, 2004; Mann \& Kelley, 2011; Fettiplace, 2017).

\subsubsection{Inner hair cell ribbon synapses}

IHCs must be able to detect sudden sound pressure changes in the environment, support incessant stimulation, and convey the signal faithfully to the SGNs. To support such high demands, the synapses between IHCs and SGNs need to maintain high rates of sustained release and are therefore equipped with ribbon synapses (Figure 1.2A). The hallmark feature of these synapses and to which they owe their name is a proteinaceous electron-dense structure called the synaptic ribbon.

The synaptic ribbon is associated with the presynaptic release sites, positioned at the basolateral plasma membrane in IHCs (Figure 1.2B). Each ribbon tethers a halo of synaptic vesicles (SVs) (Sterling \& Matthews, 2005) facilitating continuous vesicular replenishment to the release site and hence allowing an indefatigable afferent transmission at high rates with submillisecond temporal precision (Khimich et al, 2005; Matthews \& Fuchs, 2010; Wichmann $\&$ Moser, 2015). Because of this, IHCs release SVs at rates several orders of magnitude higher than conventional synapses for longer time periods (Griesinger et al, 2005). Graded variations in membrane potential - and not action potentials - induce synaptic response and influence the amount of released vesicles, a characteristic of sensory cells with ribbon synapses including IHCs, retina photoreceptors and bipolar cells (Matthews \& Fuchs, 2010). Each IHC can form 10 to 20 synapses with afferent boutons from SGNs, with numbers varying along the tonotopic region of the cochlea (Meyer et al, 2009; Fettiplace, 2017). Each IHC AZ is normally occupied by one or two ribbons and transmits information to a single SGN afferent (Fuchs et al, 2003). 


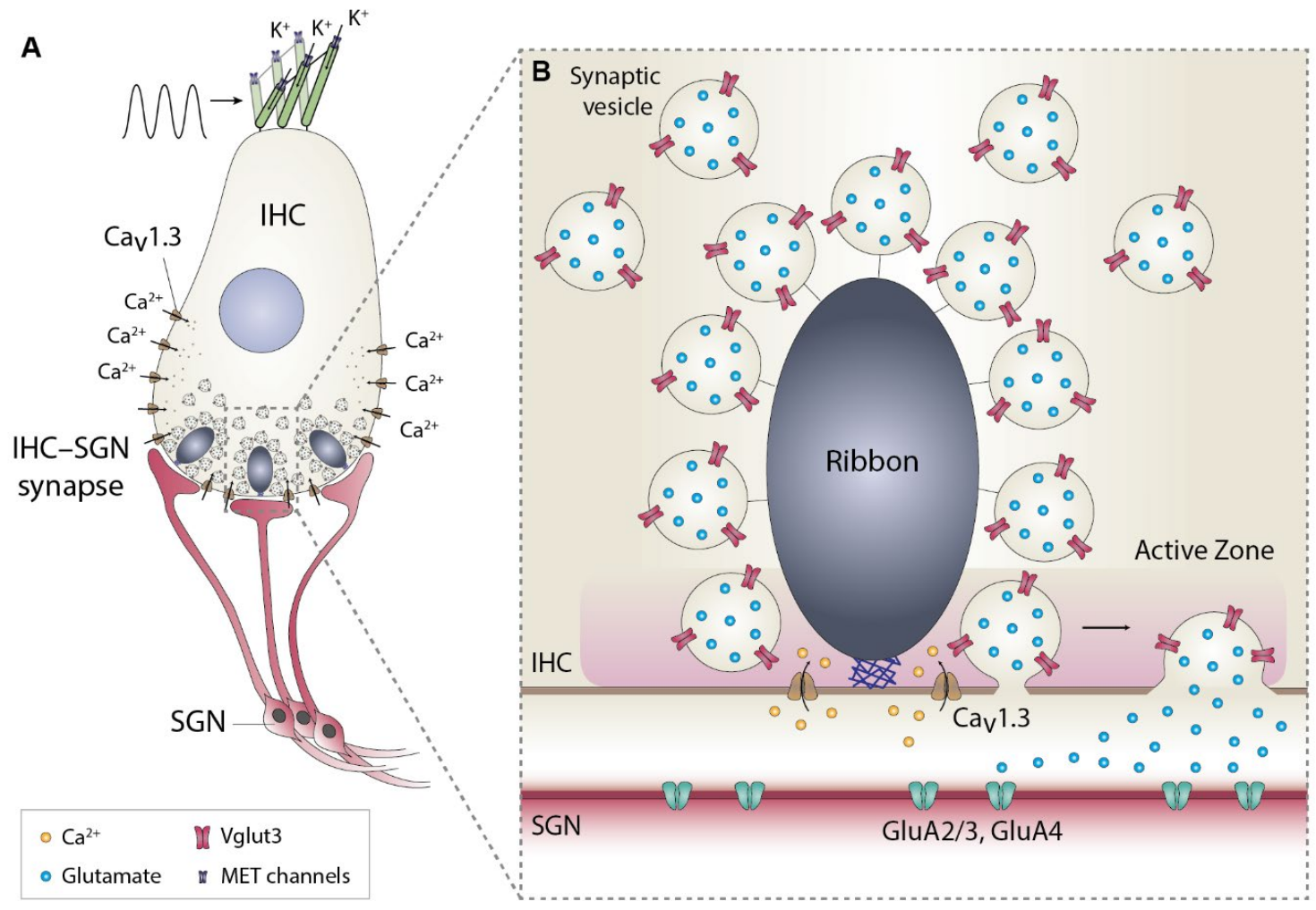

Figure 1.2. The ribbon synapse, a specialized synapse between inner hair cells (IHCs) and spiral ganglion neurons (SGNs).

Auditory ribbon synapses are highly specialized structures that assure the indefatigable encoding of sound information with sub-millisecond temporal resolution. A. Schematic representation of an IHC composed of several ribbon synapses, each connected to a single SGN. B. Schematic representation of an IHC-SGN ribbon synapse. The synaptic ribbon, mainly composed of the protein RIBEYE, tethers a large number of synaptic vesicles $(\mathrm{SVs})$ and is anchored to the presynaptic active zone membrane by the scaffolding protein bassoon. SVs undergo exocytosis upon IHC depolarization and subsequent $\mathrm{Ca}^{2+}$ influx through voltage-gated $\mathrm{Ca}^{2+}$ channels. Vglut 3 is the main glutamate transporter. The presynaptic active zone is equipped with $\mathrm{Ca}_{v} 1.3 \mathrm{Ca}^{2+}$ channels. The postsynaptic membrane contains AMPA-receptor subunits GluA2/3 and GluA4. Original illustration adapted from Moser \& Starr, 2016.

The specialized molecular anatomy of the IHC ribbon synapse, which as of now is largely unknown (see chapter 1.1.3.1), is the base for its impressive release capacity. Besides clustering $\mathrm{Ca}^{2+}$ channels at the release sites (Frank et al, 2010; Khimich et al, 2005), the ribbon delivers SVs to the AZ plasma membrane, by one of two disputed models: the "conveyor belt" model or the "safety belt" model. In the conveyor belt model the ribbon is said to operate as a conveyor belt, where it shuttles vesicles downward the ribbon toward the release sites (Lenzi \& von Gersdorff, 2001; Parsons \& Sterling, 2003; Graydon et al, 2014; Becker et al, 2018; Jean et al, 2018). In the safety belt model the ribbon slows down the process by tethering vesicles stably in mutual contact, with vesicles fusing with each other before release thus facilitating multivesicular release by compound exocytosis (Parsons \& Sterling, 2003; Matthews \& Sterling, 2008; Jackman et al, 2009). The ribbon has also been proposed to facilitate exocytosis 
through provision of multiple release sites (multi-vesicular release) by synchronization of SV fusion (Edmonds, 2004; Fuchs, 2005; Glowatzki \& Fuchs, 2002; Khimich et al, 2005). However, in late years this mechanism has been questioned, with univesicular release being proposed instead, where single SVs are released independently of each other involving glutamate release through a flickering fusion pore (Chapochnikov et al, 2014; Grabner \& Moser, 2018; Huang \& Moser, 2018).

\subsubsection{Molecular composition of inner hair cell ribbon synapses}

Collective efforts have been made to elucidate the molecular composition of IHC ribbon synapses (reviewed in Pangršič et al, 2012; Safieddine et al, 2012; Rutherford \& Pangršič, 2012; Wichmann \& Moser, 2015). While the composition of these synapses differ from that of conventional and other ribbon synapses, and the identification of an exocytic SNARE complex is still missing, there has been progress towards the identification of the ribbon components.

The main component of the ribbon is the protein RIBEYE (Schmitz et al, 2000; Schmitz, 2009), with an N-terminal A domain and a C-terminal B domain identical to the nuclear corepressor protein $\mathrm{C}$-terminal binding protein 2 (CtBP2), a transcription factor ubiquitously found in most tissues. The A domain has a predominantly structural role, whereas the $\mathrm{B}$ domain is responsible for $\mathrm{NAD}(\mathrm{H})$ binding and protein interactions with other ribbon components (Schmitz et al, 2000; Magupalli et al, 2008; Alpadi et al, 2008; Müller et al, 2019). Several other ribbon-associated proteins, present in conventional synapses, also compose the synaptic ribbons. The scaffolding protein bassoon anchors the ribbon to the presynaptic density (Frank et al, 2010). Both bassoon (Khimich et al, 2005; Frank et al, 2010; Jing et al, 2013) and RIBEYE (Frank et al, 2010; Sheets et al, 2011; Graydon et al, 2011; Maxeiner et al, 2016; Jean et al, 2018) organize individual release sites by promoting $\mathrm{Ca}^{2+}$ channel clustering at these sites, and promote vesicle replenishment to the ribbon. Piccolo/Piccolino, CtBP1, KIF3A and RIM1/2 were also identified as ribbon components (Muresan et al, 1999; Dick et al, 2001; tom Dieck et al, 2005; Deguchi-Tawarada et al, 2006; Regus-Leidig et al, 2013; reviewed in Schmitz, 2009).

While neurons use P/Q- and N-type Cav2.1/2.2 $\mathrm{Ca}^{2+}$ channels (Catterall \& Few, 2008), IHCs employ L-type Cav1.3 channels (Platzer et al, 2000; Brandt et al, 2003; Dou et al, 2004; Brandt et al, 2005) for $\mathrm{Ca}^{2+}$ influx. Additionally, they use the unconventional vesicular glutamate transporter 3 (Vglut3) to loads SVs with neurotransmitter (Seal et al, 2008; Ruel et al, 2008) as opposed to Vglut1 and Vglut2 in conventional synapses (Bellocchio et al, 2000; Fremeau et al, 2001; Takamori et al, 2001). 
We have yet to find the components of a protein complex that would make up a functional exocytic machinery in these synapses. The neuronal soluble $\mathrm{N}$-ethylmaleimide-sensitive factor attachment protein receptors (SNAREs) - SNAP-25, synaptobrevins/VAMPs 1-3 and syntaxins 1-3 - (Nouvian et al, 2011), the vesicular $\mathrm{Ca}^{2+}$ sensors synaptotagmins (Syt) 1/2 (Safieddine \& Wenthold, 1999; Beurg et al, 2010; Reisinger et al, 2011), as well as late step exocytic proteins like synaptophysins, synapsins and complexins (Safieddine \& Wenthold, 1999; Strenzke et al, 2009; Uthaiah \& Hudspeth, 2010), but also the priming proteins Munc13 and CAPS (Vogl et al, 2015) are either not expressed or are functionally redundant for exocytosis in mature IHCs. Instead, IHCs express the multi- $\mathrm{C}_{2}$ domain protein otoferlin (Roux et al, 2006; Pangršic et al, 2012), a member of the ferlin family of membrane fusion proteins (Lek et al, 2012), which appears to take over the function of many of the neuronal proteins and is currently proposed to act as the $\mathrm{Ca}^{2+}$ sensor for exocytosis (Roux et al, 2006; Vincent et al, 2014; Michalski et al, 2017) (see chapter 1.1.4). Current knowledge of the proteins present and absent in IHCs and their functional equivalents in conventional synapses is summarized in Table 1.1 .
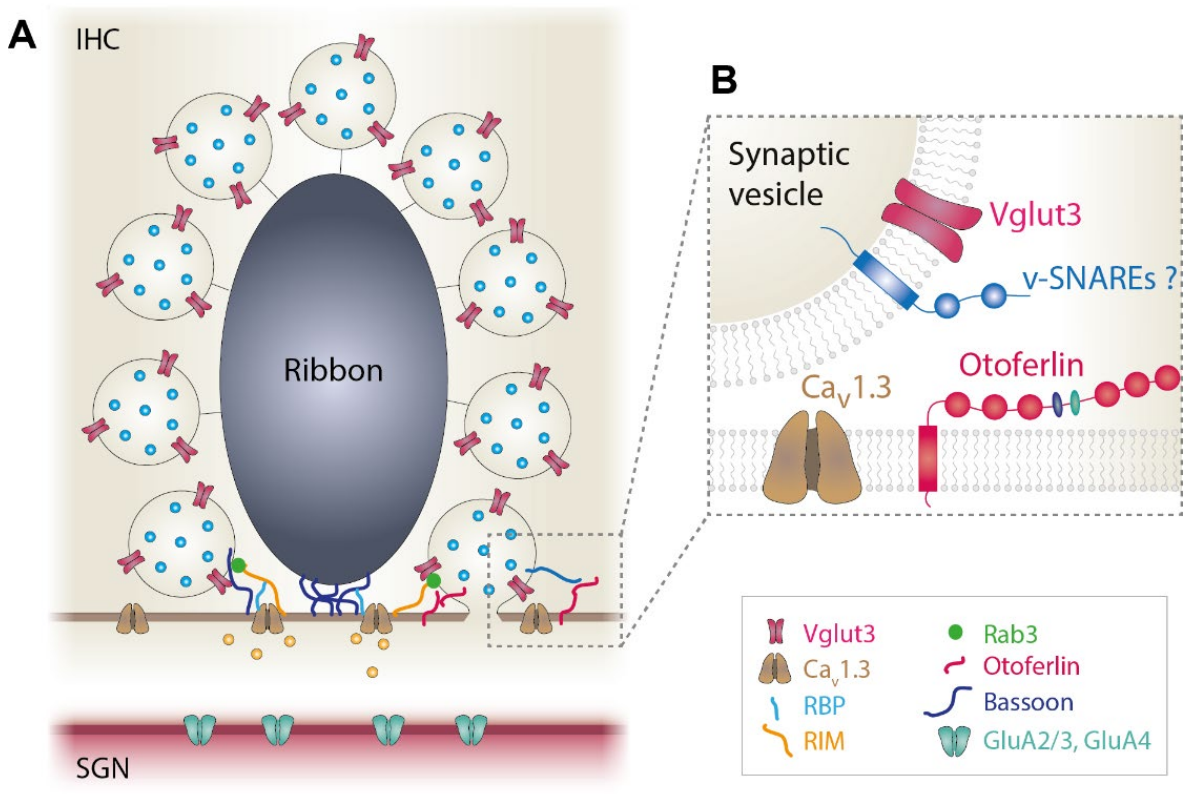

Figure 1.3. Exocytic machinery at the IHC ribbon synapse.

A. Schematic summary of the protein arrangement at mature IHC ribbon synapses. B. Vglut3 is found in IHC SVs. Otoferlin is found at the active zone (AZ) membrane. It is currently disputed whether otoferlin is found at the ribbon-associated SVs. Other vesicle-localized (v)- or target-membrane-bound (t)-SNAREs in IHCs are unknown. Original illustration adapted from Pangršič et al, 2012. 
Table 1.1. Main molecular differences between conventional synapses of the mammalian CNS and the IHC ribbon synapse.

Several synaptic proteins seem to be absent in mature IHC ribbon synapses. Instead, other proteins either specific to IHCs or found in other cell types seem to take over. The table lists first reports or reviews on the proteins for CNS and IHC synapses. "None" indicates the protein is probably absent; "FR" indicates the protein is present but is functionally redundant "?" indicates lack of published data or conflicting results. CNS, central nervous system. Adapted from Pangršič et al, 2012.

\begin{tabular}{|c|c|c|}
\hline Conventional synapses & & Cochlea inner hair cell synapses \\
\hline $\begin{array}{l}\text { Vglut1, } 2 \text { (Bellocchio et al, 2000; Fremeau et al, } \\
\text { 2001; Takamori et al, 2001) }\end{array}$ & Glutamate uptake & Vglut3 (Ruel et al, 2008; Seal et al, 2008) \\
\hline None & \multirow[t]{4}{*}{ Scaffold proteins } & Ribeye/CtBP2 (Khimich et al, 2005) \\
\hline $\begin{array}{l}\text { Bassoon (tom Dieck et al, 1998; Altrock et al, } \\
\text { 2003; Hallermann et al, 2010) }\end{array}$ & & Bassoon (Khimich et al, 2005) \\
\hline $\begin{array}{l}\text { Piccolo (Cases-Langhoff et al, 1996; Mukherjee } e t \\
\text { al, 2010) }\end{array}$ & & Piccolino (Regus-Leidig et al, 2013) \\
\hline $\begin{array}{l}\text { CAST/ELKS (Ohtsuka et al, 2002; Ohara- } \\
\text { Imaizumi et al, 2005) }\end{array}$ & & $?$ \\
\hline Rab3 (Olofsson et al, 1988; Geppert et al, 1997) & \multirow[t]{7}{*}{$\begin{array}{l}\text { Vesicle tethering, } \\
\text { docking, priming }\end{array}$} & $\begin{array}{l}\text { Rab3a, c (Uthaiah \& Hudspeth, 2010; } \\
\text { Revelo et al, 2014) }\end{array}$ \\
\hline $\begin{array}{l}\text { Synapsin (Shupliakov et al, 2011; Cesca et al, } \\
\text { 2010) }\end{array}$ & & $?$ \\
\hline RIM (Wang et al, 1997; Schoch et al, 2002) & & $\begin{array}{l}\text { RIM } 2 \alpha, \beta \text { and RIM3 } \gamma \text { (Jung et al, 2015b; } \\
\text { Picher } \text { et al, 2017b); RIM-BP2 (Krinner } \\
\text { et al, 2017) }\end{array}$ \\
\hline Munc18-1 (Hata et al, 1993; Verhage et al, 2000) & & $?$ \\
\hline $\begin{array}{l}\text { Munc13-1,2,3 (Augustin et al, 1999; Brose et al, } \\
\text { 1995; Walent et al, 1992) }\end{array}$ & & None (Vogl et al, 2015) \\
\hline CAPS (Jockusch et al, 2007) & & None (Vogl et al, 2015) \\
\hline FR (Schug et al, 2006; Reisinger et al, 2011) & & Otoferlin (Pangrsic et al, 2010) \\
\hline $\begin{array}{l}\text { Syntaxin 1, SNAP-25, Synaptobrevin } 2 \text { (Rizo \& } \\
\text { Rosenmund, 2008; Sørensen, 2009; Jahn \& } \\
\text { Scheller, 2006) }\end{array}$ & \multirow[t]{3}{*}{$\begin{array}{l}\text { Fusion and regulation of } \\
\text { fusion }\end{array}$} & $\begin{array}{l}\text { None (Safieddine \& Wenthold, 1999; } \\
\text { Uthaiah \& Hudspeth, 2010; Nouvian } e t \\
a l, 2011 \text { ) }\end{array}$ \\
\hline $\begin{array}{l}\text { Synaptotagmin 1,2 (Matthew et al, 1981; Perin et } \\
\text { al, 1990; Geppert et al, 1991, 1994) }\end{array}$ & & $\begin{array}{l}\text { Otoferlin (Pangrsic et al, 2010) } \\
\text { Synaptotagmin } 4 \text { (Johnson et al, 2010) }\end{array}$ \\
\hline $\begin{array}{l}\text { Complexins 1-4 (Takahashi et al, 1995; } \\
\text { McMahon et al, 1995; Reim et al, 2001) }\end{array}$ & & $\begin{array}{l}\text { None (Strenzke et al, 2009; Uthaiah \& } \\
\text { Hudspeth, 2010) }\end{array}$ \\
\hline $\begin{array}{l}\text { P/Q- and N-type Cav2.1/2.2 (Catterall \& Few, } \\
\text { 2008; Catterall, 2011) }\end{array}$ & \multirow[t]{3}{*}{$\begin{array}{l}\mathrm{Ca}^{2+} \text { channel and its } \\
\text { regulation }\end{array}$} & $\begin{array}{l}\text { L-type Cav1.3 (Platzer et al, 2000; Brandt } \\
\text { et al, 2003; Dou et al, 2004; Brandt et al, } \\
\text { 2005) }\end{array}$ \\
\hline CaBP1 (Lee et al, 2002) & & $\begin{array}{l}\text { CaBP2 (Yang et al, 2006; Cui et al, 2007; } \\
\text { Yang et al, 2016; Picher et al, 2017a) }\end{array}$ \\
\hline$?$ & & Harmonin (Gregory et al, 2011) \\
\hline $\begin{array}{l}\text { AP-2 (Keen, 1987; Kirchhausen et al, 1989; } \\
\text { Boucrot et al, 2010) }\end{array}$ & \multirow[t]{6}{*}{$\begin{array}{l}\text { Endocytosis and SV } \\
\text { reformation }\end{array}$} & $\begin{array}{l}\text { AP-2 (Duncker et al, 2013; Jung et al, } \\
\text { 2015a) }\end{array}$ \\
\hline AP180 (Keen, 1987; Zhang et al, 1998) & & AP180 (unpublished) \\
\hline Synaptotagmin (Zhang et al, 1994) & & $?$ \\
\hline $\begin{array}{l}\text { Endophilins (Masuda et al, 2006; Bai et al, 2010; } \\
\text { Milosevic et al, 2011) }\end{array}$ & & Endophilin A1-3 (Kroll et al, 2019) \\
\hline $\begin{array}{l}\text { Dynamins 1, 2, } 3 \text { (Cao et al, 1998; Ferguson } \text { et } \\
\text { al, 2007; Ferguson \& De Camilli, 2012) }\end{array}$ & & Dynamins 1-3 (Neef et al, 2014) \\
\hline FR (Schug et al, 2006; Reisinger et al, 2011) & & Otoferlin (Strenzke et al, 2016) \\
\hline
\end{tabular}




\subsubsection{Synaptic vesicle pool organization and synaptic vesicle cycle at inner hair cell ribbon synapses}

Electron microscopy and electrophysiological capacitance measurements resulted in distinct classifications of SV pools in different synapses. In conventional synapses of the central nervous system (CNS), three main pools of SVs were characterized morphologically and physiologically: i) readily releasable pool (RRP), consists of vesicles docked at the AZ membrane and primed for release; ii) recycling pool or slowly releasable pool (SRP), located in the vicinity of the AZ membrane, mostly refilled by newly endocytosed SVs and refills the RRP; iii) reserve pool, located further away from the AZ membrane, formerly seem as the supplier for the refilling of the recycling and RRP pools (Rizzoli \& Betz, 2005; Denker \& Rizzoli, 2010) but recently proposed to be static (Truckenbrodt et al, 2018) (Figure 1.4A).

The different architecture of the ribbon synapses led to an adapted vesicle pool organization. In these synapses, SVs are connected to the ribbon and to the AZ plasma membrane by filaments also termed "tethers". Morphologically, the SV pools are then subdivided into: i) membrane-proximal SV (MP-SV) pool, in direct vicinity to the presynaptic density and at a distance of $\leq 40 \mathrm{~nm}$ from the $\mathrm{AZ}$ membrane, composed of docked, tethered and non-tethered vesicles sitting on the plasma membrane,; ii) ribbon-associated SV (RA-SV) pool, the first row of SVs around the ribbon except MP-SVs (at a distance of $\leq 80 \mathrm{~nm}$ from the ribbon), tethered and non-tethered to the ribbon; iii) outlying or cytosolic SV pool, at a distance of $\geq 80 \mathrm{~nm}$ from the ribbon, all SVs not belonging to the MP-SV and RA-SV pools (Kantardzhieva et al, 2013; Chakrabarti et al, 2018). Capacitance measurements recorded from IHCs revealed two kinetic components of exocytosis: a fast component, occurring at high release rates for up to $-15 \mathrm{~ms}$ but slowing down after a few milliseconds of stimulation, followed by a slower component, occurring at a nearly constant rate between 20 and $500 \mathrm{~ms}$ (Moser \& Beutner, 2000; Schnee et al, 2011). The SV pools have thus been classified physiologically based on dynamics and release kinetics into: i) readily releasable pool (RRP), the population of SVs located just above the $\mathrm{AZ}$ membrane and that can be immediately released upon depolarization and $\mathrm{Ca}^{2+}$ influx, reflects the fast component of exocytosis for short IHC depolarizations (up to $-15 \mathrm{~ms}$ ); ii) recycling pool, further away from the AZ membrane and refills the RRP; iii) reserve pool, composed of free cytosolic vesicles and is the largest pool and constantly refills the recycling and RRP pools, and it represents the sustained component of exocytosis for long IHC depolarizations; iv) distant pool, serves as a reservoir to refill all other pools. Movement of SVs between pools is believed to be dynamic (Moser \& Beutner, 2000; Beutner \& Moser, 2001; Nouvian et al, 2006; Pangrsic et al, 2010; Schnee et al, 2011; Michalski et al, 2017) (Figure 1.4.C). In conventional synapses, exocytosis and movement of SVs between pools is regulated by second messenger-activated protein kinases, like CaMKII $\delta$ and PKC $\alpha$ (see chapter 1.2). As of now, in IHC synapses only otoferlin, involved in several steps of the SV cycle, is known to be regulated by this kind of mechanism (Meese et al, 2017). 

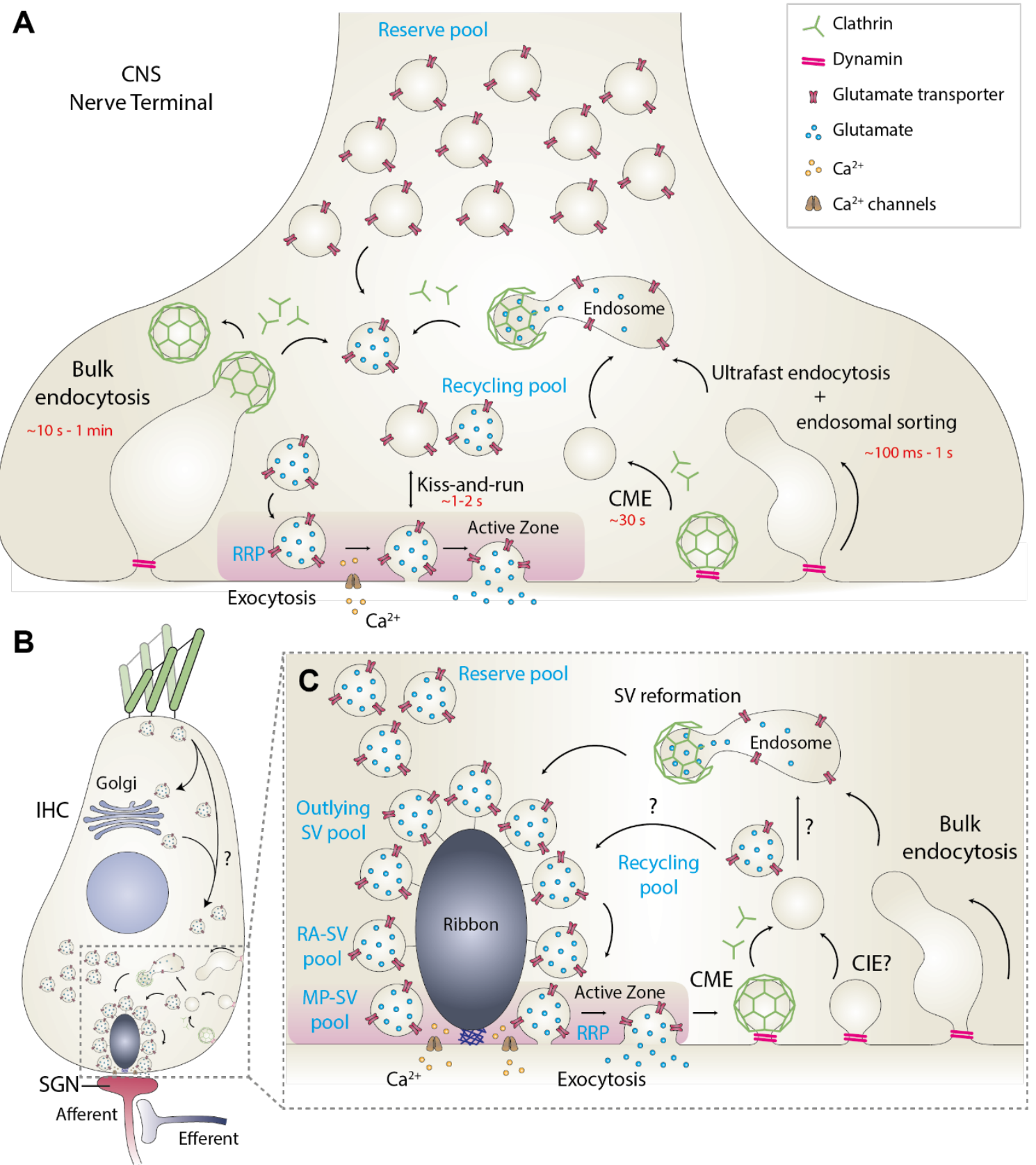

Figure 1.4. Synaptic architecture, synaptic vesicle pools and mechanisms of synaptic vesicle recycling at conventional synapses and auditory ribbon synapses.

A. Schematic representation of a central nervous system (CNS) nerve terminal. Displayed are the different pools of synaptic vesicles (SVs): i) the readily releasable pool (RRP), consisting of SVs docked at the active zone (AZ) membrane and ready for release; ii) the recycling pool refills the RRP and is located close to the AZ membrane; iii) the reserve pool refills the recycling and RRP pools. Current understanding of exocytic and endocytic mechanisms at this synapse is represented. SVs directly in contact with the AZ plasma membrane fuse (exocytosis) and this process is followed by membrane internalization (endocytosis) via i) clathrin-mediated endocytosis (CME), at distal sites, requiring the assembly of a protein coat composed of clathrin and adaptor proteins to induce curvature and form a spherical invagination; ii) Kiss-and-run: SV fusion pore opening and closing at the AZ; iii) bulk endocytosis, at distal sites, where a larger area of membrane is internalized as cisternae or endosomes from which multiple SVs can bud off using CME or clathrin-independent endocytosis (CIE); iii) ultrafast endocytosis, with reformation of SVs from clathrin-coated pits from endosomes (Watanabe \& Boucrot, 2017). B. Schematic representation of an inner hair cell (IHC) with different modes of synaptic vesicle trafficking: i) constitutive membrane trafficking takes place in the top and nuclear regions of the IHC, with endocytosed material being converted to large vesicles and early endosome-like structures; ii) SV recycling happens at the base 
of the IHC in the vicinity of the synaptic ribbon and it involves the formation of large membrane infoldings and cisternae that give rise to SVs (Revelo et al, 2014). C. Schematic representation of the SV cycle at IHC ribbon synapses. The different SV pools are represented. Ribbon-tethered SVs are delivered to and fuse with the AZ plasma membrane undergoing exocytosis. Membrane and exocytic machinery are recycled via CME and bulk endocytosis. Endocytosed material fuses with large endosomal compartments in close proximity to the synaptic ribbons. SV reformation occurs from clathrin-coated pits in large endosomal compartments or possibly directly from newly endocytosed material, which in turn replenish SVs to the ribbon.

After SV exocytosis, membrane content and exocytic machinery content are recycled from the release sites at the AZ membrane to generate new docking spots for new-coming SVs that are transported along the ribbon. This membrane retrieval assures a constant SV turnover and occurs via compensatory endocytic mechanisms. This process is thought to be mostly mediated by clathrin-mediated endocytosis (CME) or bulk endocytosis, depending on the intensity of the stimulus (Beutner et al, 2001; Neef et al, 2014; Jung et al, 2015a; Michalski et al, 2017). Two studies in particular sought to analyze endocytic intermediates and their processing into vesicles throughout the IHCs (Kamin et al, 2014; Revelo et al, 2014). The authors propose that in IHCs constitutive membrane trafficking is abundant and takes place both at rest and during stimulation, and most endocytosed material converts into tubular organelles in the top and nuclear areas that later give rise to large vesicles that resemble early endosomes (Figure 1.4B). Synaptic vesicle recycling takes place after stimulation at the base of the cell where the AZs are located. Here, synaptic vesicles tethered to synaptic ribbons are released. During recovery after IHC stimulation, membrane material is recycled via endocytosis which depending on the stimulus intensity results in the formation of i) clathrin-coated vesicles via CME (mild stimulus); ii) bulk endosomes (medium strength stimulus); and iii) large cisterns (strong stimulus). Bulk endosomes and large cisterns are later converted to small vesicles (Figure 1.4B-C). It was also shown that in some cases strong stimulation leads to the formation of large cisterns as large as $-450 \mathrm{~nm}$ in diameter and situated close to the AZs (Strenzke et al, 2016). It is possible that these structures result from ultrafast endocytosis like in hippocampal synapses where strong stimuli trigger ultrafast endocytosis resulting in the formation of endosomes about four times the size of SVs (Watanabe et al, 2013).

IHC SVs seem not to differ in size (Neef et al, 2007b, 2014; Michanski et al, 2019) from the average SV (Harris \& Sultan, 1995; Hu et al, 2008; Qu et al, 2009) (average: 40 nm diameter; most in the range 30-50 $\mathrm{nm}$ diameter). Bulk endocytosis appears to contribute to the formation of larger vesicles (50-70 $\mathrm{nm}$ diameter) and endosome-like vacuoles (ELVs) (>70 nm diameter) (Chakrabarti et al, 2018). Properly-sized SVs are more likely to be formed from i) clathrincoated pits in these larger endosome-like structures either located near the ribbon and that subsequently "feed" the ribbon (Revelo et al, 2014; Jung et al, 2015a; Strenzke et al, 2016; Kroll et al, 2019) or ii) directly from newly endocytosed material, like large membrane invaginations and cisterns found at the AZ membrane, via a clathrin-dependent or clathrinindependent pathway (Neef et al, 2014; Jung et al, 2015a). However, it is not known if newly 
internalized membrane first fuses with bona fide endosomes or if it is directly compartmentalized (Figure 1.4C). As in conventional synapses, the proteins clathrin, dynamin, amphiphysin (Neef et al, 2014), the adaptor protein complex 2 (AP-2) (Duncker $e t$ al, 2013; Jung et al, 2015a), and endophilin A (Kroll et al, 2019) are involved in CME in IHCs. AP-2 and endophilin A were shown to be also involved in clathrin-dependent SV reformation and AZ clearance (Duncker et al, 2013; Jung et al, 2015a; Kroll et al, 2019).

\subsubsection{Otoferlin}

Mature IHC synapses lack the SV proteins Syt1 and Syt2 (Safieddine \& Wenthold, 1999; Beurg et al, 2010; Reisinger et al, 2011), which function as $\mathrm{Ca}^{2+}$ sensors for transmitter release at CNS synapses (Geppert et al, 1991, 1994; Südhof, 2013). Synaptotagmins contain two cytoplasmic $\mathrm{C}_{2}$ domains and bind to membrane phospholipids in a $\mathrm{Ca}^{2+}$-dependent manner (Brose et al, 1992; Sutton et al, 1995; Wang et al, 2014), triggering the last steps of exocytosis via interaction with the SNARE complex (Bennett et al, 1992; Söllner et al, 1993; Li et al, 1995; Giraudo et al, 2006; Südhof, 2013). Unlike CNS synapses, IHC ribbon synapses contain otoferlin, a multi- $\mathrm{C}_{2}$ domain protein belonging to the ferlin family of proteins (Lek et al, 2010, 2012). Mutations disrupting the OTOF gene lead to a form of autosomal recessive nonsyndromic hearing loss in humans, DFNB9, with severity ranging from moderate-to-profound depending on the mutation (Yasunaga et al, 1999; Varga et al, 2003; Shearer \& Smith, 2015) (Figure 1.5A).

\subsubsection{Structure}

The ferlin protein family is composed of six members in mammals: dysferlin (Fer1L1), otoferlin (Fer1L2), myoferlin (Fer1L3), Fer1L4, Fer1L5, and Fer1L6. All ferlins contain six to seven $C_{2}$ domains sharing high sequence homology (Jiménez \& Bashir, 2007), a highly conserved FerI motif between $\mathrm{C}_{2} \mathrm{~B}$ and $\mathrm{C}_{2} \mathrm{C}$ domains, and a C-terminal transmembrane domain. $C_{2}$ domains consist of a $\beta$-sandwich structure composed of eight anti-parallel $\beta$ strands with connecting top loops predicted to bind $\mathrm{Ca}^{2+}$ ions. They are $\mathrm{Ca}^{2+}$-dependent membrane-targeting modules found in many proteins involved in signal transduction or membrane trafficking, as is the case of phospholipases, protein kinase C (PKC), synaptotagmins, and ferlins (Nalefski \& Falke, 1996; Cho \& Stahelin, 2006). In fact, ferlins were shown to regulate $\mathrm{Ca}^{2+}$-induced membrane fission and fusion events (Lek et al, 2012; Johnson, 2017). To date, due to technical hurdles related to the size, complexity, and instability of otoferlin, only the structure of its $\mathrm{C}_{2} \mathrm{~A}$ domain was solved (Helfmann et al, 2011), and a putative model of the FerA domain based on dysferlin's FerA domain was created (Harsini et al, 2018). 


\subsubsection{Isoforms}

Several otoferlin variants have been reported in different tissues. A long variant $-7 \mathrm{~kb}$-long was detected in human and mouse brain, while a shorter $-5 \mathrm{~kb}$-long variant was present in human heart, placenta, liver, pancreas, skeletal muscle, kidney, inner ear and brain tissues but was absent in mouse (Yasunaga et al, 1999, 2000). The long otoferlin variant (1997-amino acidslong) consists of six $\mathrm{C}_{2}$ domains $\left(\mathrm{C}_{2} \mathrm{~A}-\mathrm{F}\right)$, possibly a seventh $\mathrm{C}_{2}$ domain $\left(\mathrm{C}_{2} \mathrm{de}\right)$ predicted between the $\mathrm{C}_{2} \mathrm{D}$ and $\mathrm{C}_{2} \mathrm{E}$ domains, a FerA domain, a FerB domain and a C-terminal transmembrane domain (Yasunaga et al, 1999, 2000; Roux et al, 2006; Lek et al, 2010, 2012; Pangršič et al, 2012; Harsini et al, 2018) (Figure 1.5A). A shorter variant containing only the $\mathrm{C}_{2} \mathrm{D}-\mathrm{F}$ and transmembrane domains was also reported (Yasunaga et al, 1999, 2000).

\subsubsection{Expression and distribution}

Like other ferlins and the SNAREs synaptobrevin and syntaxin, otoferlin also belongs to the family of tail-anchored (TA) proteins which contain their transmembrane domain close to the C-terminus while the $\mathrm{N}$-terminus is oriented towards the cytoplasm (Kalbfleisch et al, 2007). These proteins reside in several intracellular compartments like secretory organelles and the plasma membrane. The insertion of these proteins into the membrane of the endoplasmic reticulum (ER) is done post-translationally and is mediated by the guided entry of TA proteins (GET)/TRC40/Asnal pathway, with the involvement of the tryptophan-rich basic protein (WRB) and the calcium-modulating cyclophilin ligand (Caml) as the TRC40 receptor at the ER (Vilardi et al, 2011; Yamamoto \& Sakisaka, 2012). WRB knock-out (WRB ${ }^{-1}$ ) mouse IHCs showed reduced otoferlin levels and disruption of synaptic structure and function, which ultimately resulted in hearing impairment (Vogl et al, 2016).

Otoferlin's expression varies among different cell types and changes during development. Otoferlin is expressed in auditory HCs as early as embryonic day (E) 16 in IHCs and E18 in OHCs, reaching its maximal expression at postnatal day (P) 6 in both cell types; in OHCs the expression of otoferlin decreases after P6 and is almost abolished with maturation, whereas IHCs continue expressing the protein (Roux et al, 2006; Beurg et al, 2010; Pangrsic et al, 2010; Strenzke et al, 2016) (Figure 1.5B). Otoferlin is essential for $\mathrm{Ca}^{2+}-$ evoked exocytosis in IHCs after $\mathrm{P} 4$, in contrast to early developmental stages where exocytosis is otoferlin-independent (Beurg et al, 2010). Ultrastructural analysis via immunogold electron microscopy (EM) with post-embedding showed that in IHCs otoferlin localizes to the plasma membrane and synaptic vesicles (tethered and non-tethered to the ribbon) (Roux et al, 2006). By contrast, in another study using immunogold EM with pre-embedding, no immunogold particles were detected in SVs tethered to the ribbon (Strenzke et al, 2016) (Figure 1.5C2,C4-5). Strenzke and collaborators also found otoferlin in vesicular structures ranging from -50 to $450 \mathrm{~nm}$ in diameter, with the largest most likely representing ELVs (Figure 1.5C). 
Immunohistochemistry stainings additionally revealed that otoferlin is expressed not only at the presynaptic area but also at the apical region of IHCs above the nucleus where the Golgi apparatus is located (Schug et al, 2006; Heidrych et al, 2008) (Figure 1.5B), where it colocalized with the trans-Golgi markers GM130 and TGLON2 (Redpath et al, 2015). Additionally, otoferlin was reported to colocalize and interact with Rab8b (Heidrych et al, 2008), a protein that regulates the trafficking along the trans-Golgi network, the endosome recycling pathway and basolateral transport of SVs in polarized epithelial cells (Henry \& Sheff, 2008).

\subsubsection{Function and interaction partners}

Multiple converging fields of evidence, with great contribution from different mutant mouse lines, place otoferlin as a major key player in several steps of the IHC synaptic vesicle cycle.

Otoferlin knock-out mice (Otof ${ }^{--}$) are profoundly deaf, with almost entirely abolished IHC exocytosis albeit normal $\mathrm{Ca}^{2+}$ currents, ribbon morphogenesis and SV numbers (Roux et al, 2006; Reisinger et al, 2011; Vogl et al, 2015). In light of this evidence, it was proposed that otoferlin is essential for a late step of exocytosis of the RRP of vesicles, likely priming and/or fusion. It was shown that Syt 1 and otoferlin cannot replace each other, since neither virusmediated Syt 1 expression in tof $^{-/}$IHCs nor ectopic expression of otoferlin in Syt1-defficient chromaffin cells and neurons restored exocytosis (Reisinger et al, 2011). This led to the hypothesis that otoferlin is the main $\mathrm{Ca}^{2+}$ sensor that triggers exocytosis in mature IHCs.

Since the synapses of $\mathrm{Otof}^{-1-}$ mice are silent, the exact role of otoferlin and at which steps of the synaptic vesicle cycle it acts cannot be determined using this model. Several mutant mouse lines were generated to assist in this task. The pachanga mouse model (Otof ${ }^{\text {Pgal }{ }^{\text {gga }} \text { ), harboring }}$ the p.Asp1767Gly (D1767G) missense mutation in the $\mathrm{C}_{2} \mathrm{~F}$ domain of otoferlin and also profoundly deaf (Schwander et al, 2007), presented some residual otoferlin expression in IHCs and unaffected vesicle fusion (RRP exocytosis) but showed lower rates of vesicle replenishment (sustained exocytosis) (Pangrsic et al, 2010). It was then postulated that otoferlin is important for SV replenishment, providing an explanation for the fast SV replenishment rates in IHCs. Some OTOF mutations cause temperature-sensitive auditory synaptopathy/neuropathy, as is the case of the p.Ile515Thr mutation in otoferlin's $\mathrm{C}_{2} \mathrm{C}$ domain (Mirghomizadeh et al, 2002; Varga et al, 2006), where at normal core body temperatures "compound heterozygous" patients for this mutation (one allele carries the missense mutation and the other an "inactivating" premature STOP codon) display normal-to-mild hearing impairment, with mild elevation of auditory thresholds and impairment of speech perception, but suffer from severe-to-profound deafness at elevated body temperature (Starr et al, 1996; Varga et al, 2006; Shearer \& Smith, 2015). The Otof I515T/515T knock-in mouse model, homozygous for this mutation, showed moderate hearing impairment, with reduced otoferlin levels, enlarged SVs possibly of 
endosomal origin and strongly reduced exocytosis for long stimuli (Strenzke et al, 2016). Immunogold labeling revealed the presence of otoferlin in large ELVs found in AP-2 $\mu$ deficient IHCs (Jung et al, 2015a). It became evident that otoferlin is essential for the reformation of properly-sized and fusion-competent vesicles. An additional role for otoferlin in SV endocytosis via the reported interactions with AP-2 and endophilin A (Duncker et al, 2013; Jung et al, 2015a; Kroll et al, 2019) is probable.

Different studies reported that up to five of otoferlin's $\mathrm{C}_{2}$ domains are able to bind $\mathrm{Ca}^{2+}$ and phosphatidylinositol-4,5-bisphosphate (PI(4,5) $\mathrm{P}_{2}$ or $\mathrm{PIP}_{2}$ ) (Roux et al, 2006; Ramakrishnan et al, 2009; Goodyear et al, 2010; Johnson \& Chapman, 2010; Helfmann et al, 2011; Padmanarayana et al, 2014; Meese et al, 2017; Michalski et al, 2017). However, it is currently disputed which of the domains actually bind $\mathrm{Ca}^{2+}$, a topic vastly discussed in chapter 4.2.2.

Several lines of evidence indicate that the long variant of otoferlin is crucial for proper synaptic transmission both in auditory IHCs (Roux et al, 2006; Pangrsic et al, 2010) and vestibular HCs (Dulon et al, 2009). Of interest, truncated otoferlin versions retaining the $\mathrm{C}_{2} \mathrm{~F}$ domain could not fully restore exocytosis in mouse Otof $^{-1-}$ IHCs (Tertrais et al, 2019).

Otoferlin might also be involved in the tethering of SVs to the AZ membrane during exocytosis, as these tethers were reported to be altered in Otof $^{-1-}$ IHCs (Vogl et al, 2015). Otof Pgal/ga IHCs, with a defect in sustained release (Pangrsic et al, 2010), showed multi-tethered SVs and docked SVs at the AZ membrane (Chakrabarti et al, 2018) pointing toward a role for otoferlin in release site clearance. Kroll et al, 2019 proposed that the interaction of otoferlin with endophilin $\mathrm{A}$ is required for this purpose.

No morphological or ribbon number differences were observed at P6 between Otof ${ }^{-/-}$and wild-type IHCs, indicating that otoferlin has no involvement in IHC development and survival or in ribbon formation (Roux et al, 2006). Otoferlin seems, however, to be important for ribbon synapse maintenance after the onset of hearing, since P15 Otof ${ }^{-/-}$and Otof ${ }^{\text {Pgal }{ }^{g a a}} \mathrm{IHCs}$ showed $-40 \%$ and $-19 \%$ reduction in ribbon synapse numbers, respectively, when compared to wild-type IHCs (Roux et al, 2006; Pangrsic et al, 2010). Otoferlin might also be important for synapse maturation: i) gene delivery of otoferlin at P6-P7 revealed to be too late to reverse or prevent synaptic ribbon loss in Otof ${ }^{-1-}$ IHCs, with dual-AAV transduced and nontransduced Otof ${ }^{-/-}$IHCs showing equal synaptic ribbon numbers at P26-29 (Al-Moyed et al, 2019); ii) Otof ${ }^{-1-}$ IHCs present a delay in synapse maturation with higher synapse numbers than wild-type IHCs of the same age (up until P14) (Al-Moyed, 2019).

Exocytic responses of both the RRP and recycling pool components in IHCs are governed by Cav1.3 channels and require otoferlin (Roux et al, 2006; Pangrsic et al, 2010; Levic et al, 2011; Vincent et al, 2014). Cav1.3 channels and otoferlin were proposed to interact physically in IHCs (Ramakrishnan et al, 2009; Hams et al, 2017). Additionally, Vincent and collaborators showed that otoferlin controls the ratio between fast and inactivating Cav1.3 isoforms, 
indicating that otoferlin influences $\mathrm{Ca}^{2+}$ influx dynamics in IHCs (Vincent et al, 2014, 2017). Recently, Johnson et al, 2017 reported that the coupling between Cav1.3 channels and the $\mathrm{Ca}^{2+}$ sensor (e.g. otoferlin) varies tonotopically along the cochlea, with high-frequency cells being more microdomain (for better encoding of a large dynamic range of sound intensities) and low-frequency cells operating via $\mathrm{Ca}^{2+}$ nanodomains (for precise time encoding) (Johnson et al, 2017). While it cannot be ruled out that another yet-to-be-identified $\mathrm{Ca}^{2+}$-sensing protein might assist otoferlin, IHCs seem to use otoferlin as the main $\mathrm{Ca}^{2+}$ sensor possibly in different steps of the SV cycle as proposed by Michalski et al, 2017.

The filamentous actin (F-actin) network seems to control otoferlin-dependent exocytosis in auditory IHCs (Vincent et al, 2015; Guillet et al, 2016) by forming dense cage-shaped structures beneath the synaptic ribbons that maintain a tight spatial organization of Cav1.3 channels at the synaptic ribbons (Vincent et al, 2015). Each F-actin cage associates with one ribbon and one Cav1.3 channel immunoreactive patch, and colocalizes with otoferlin (Vincent et al, 2015), predicting a physical association of otoferlin with the F-actin network either directly or via scaffolding protein(s).

Otoferlin was also reported to colocalize with endosomal (EEA1) and Golgi proteins (GM130) which led to a yeast-two-hybrid screen that retrieved the GTPase Rab8b as interaction partner of otoferlin in IHCs (Heidrych et al, 2008). As already mentioned, Rab8b regulates the trafficking along the trans-Golgi network, the endosome recycling pathway but also controls the basolateral transport of SVs in polarized epithelial cells (Henry \& Sheff, 2008). This supports the notion that otoferlin is involved in recycling of endosomes into SVs and suggests an additional role for otoferlin in trafficking events in IHCs. The unique motor myosin VI, involved in the early endocytic pathway and also required for cargo sorting (Tumbarello et al, 2013) not only interacts with otoferlin (Roux et al, 2009; Heidrych et al, 2009) but, like all myosin motors, also associates with the actin filaments by moving along them, thereby regulating the dynamics of the cytoskeleton and affecting transport of cellular components. It is currently hypothesized that myosin VI, F-actin and otoferlin are involved in endosomal trafficking processes in IHCs.

Although it was shown that otoferlin is able to bind syntaxin 1 and SNAP-25 in vitro (Roux et al, 2006; Ramakrishnan et al, 2009, 2014; Hams et al, 2017), these proteins seem to be absent from mature IHC synapses (Nouvian et al, 2011) and it is not known if these interactions are of physiological relevance.

Otoferlin emerges as a multi-functional protein, being essential to many processes in IHCs like exocytosis, SV replenishment, SV reformation, endocytosis and exo-endocytosis coupling. 
A
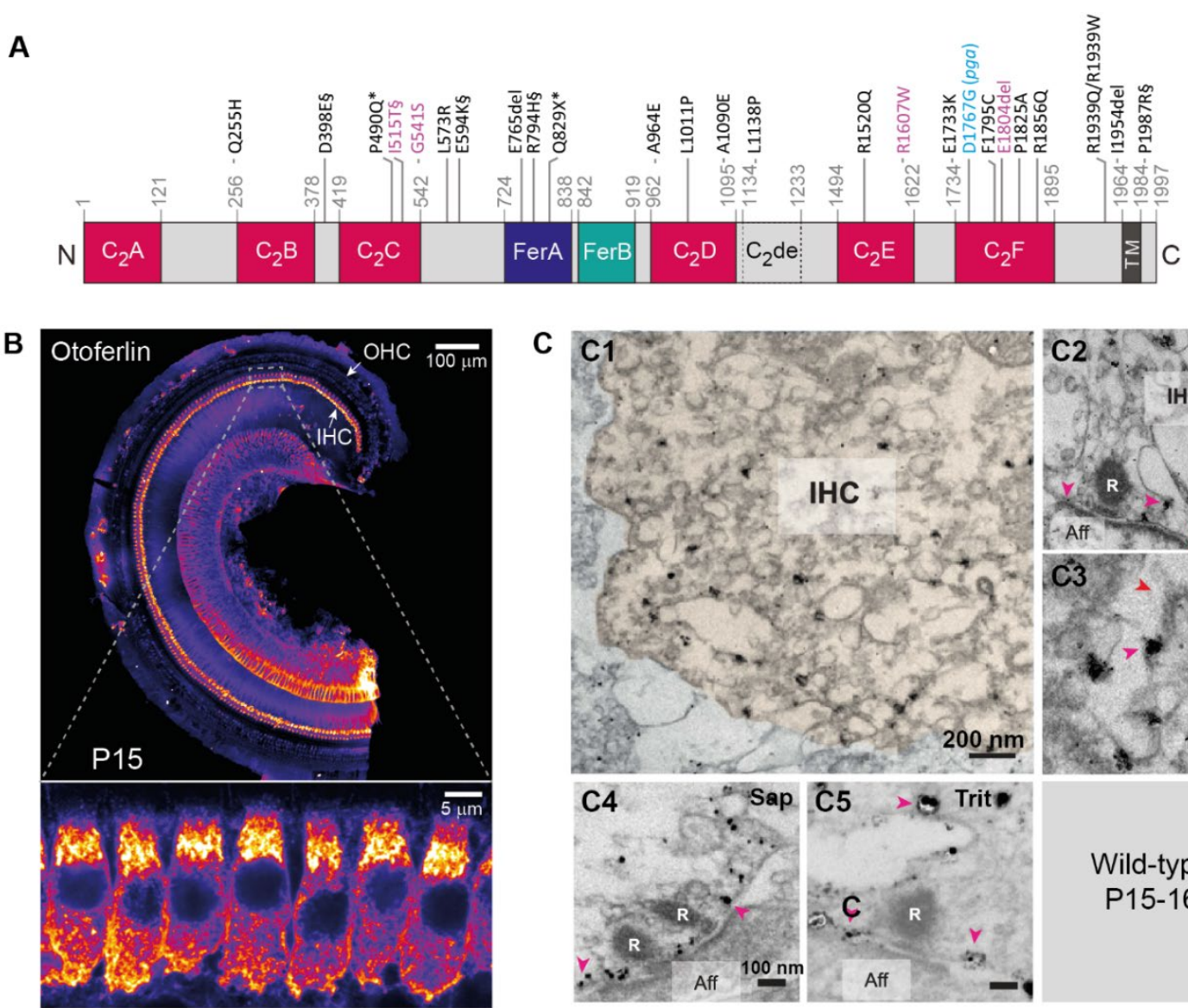
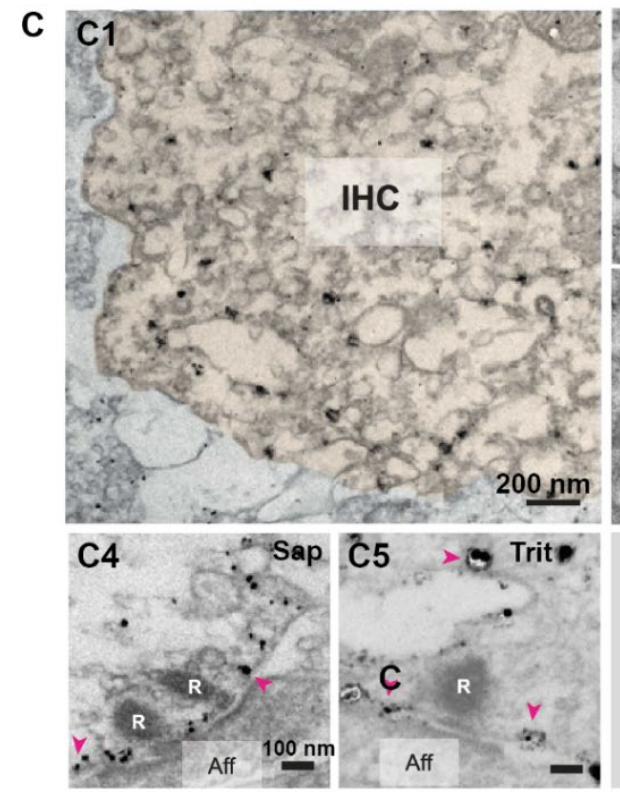

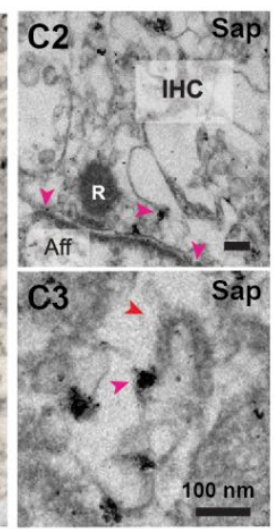

Wild-type

P15-16

D

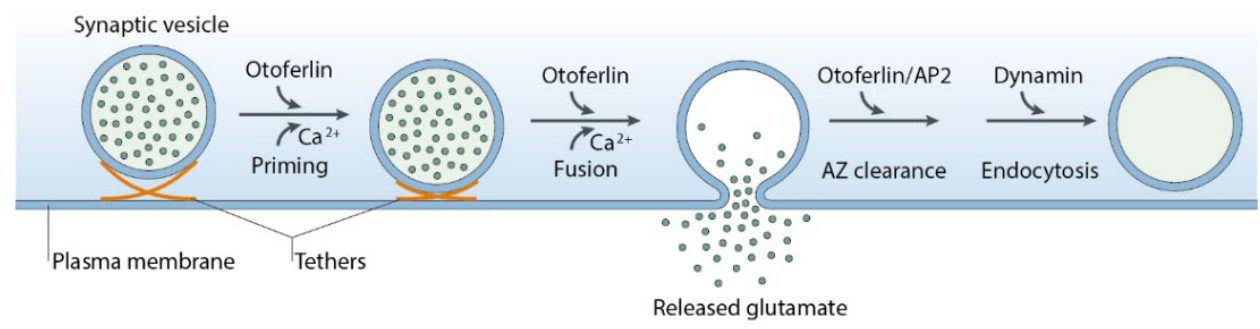

Figure 1.5. Otoferlin's structure, distribution and function in IHCs.

A. Schematic representation of the long isoform of human otoferlin present in auditory IHCs. Protein domain structure, with pathogenic missense mutations and in-frame deletions (top). $\mathrm{C}_{2}$ domains $\left(\mathrm{C}_{2} \mathrm{~A}-\mathrm{F}, \mathrm{C}_{2} \mathrm{de}\right)$, FerA and FerB domains and boundaries are depicted. Missense mutations linked to temperature sensitive hearing impairment like the p.Ile515Thr (I515T) mutation are displayed in magenta. The p.Asp1767Gly (D1767G) missense mutation is depicted in light blue. Adapted from Pangršič et al, 2012; Harsini et al, 2018. B. Wild-type organ of Corti (P15) immunolabeled for otoferlin (intensity-coded lookup table) (top). High magnification views of IHCs (bottom). Original unpublished data. C. Otoferlin's subcellular localization in wild-type P15-16 IHCs visualized by immunogold EM. C1, Random ultrathin sections through the basal part of the IHCs. C2-C5, Magnified synaptic ribbons (R) and postsynaptic afferent boutons of SGNs (Aff). Note otoferlin immunogold labelling at active zone membranes and endosomal compartments (pink arrowheads) but not around the ribbon. Treatments with saponin (Sap) (C2-C4) or Triton X-100 (Trit) (C5). Adapted from Strenzke et al, 2016. D. Summary of different roles of otoferlin in IHC synaptic transmission: vesicle fusion and vesicle replenishment, active zone clearance, endocytosis. From Moser \& Starr, 2016. IHC: inner hair cell; Fer, Ferlin-specific motif; TM, transmembrane domain; AZ: active zone. 


\subsection{CaMKII and PKC as regulators of synaptic transmission}

Protein phosphorylation and dephosphorylation modulates synaptic transmission by regulating long-term synaptic plasticity but are also directly involved in modulating exocytosis in neurons and other cell types. The control of the SV cycle, either by increasing the number of SVs being reformed from the reserve pool or by increasing the number of available SV ready to fuse, is one of the ways to regulate neurotransmitter release and underlies some forms of synaptic plasticity (Südhof, 1995).

At conventional synapses, protein interactions within the presynaptic release apparatus are regulated via phosphorylation of various exocytic proteins (Turner et al, 1999). The final steps of SV exocytosis are regulated by second messenger-activated protein kinases expressed in presynaptic terminals. In particular, the activation of $\mathrm{Ca}^{2+} /$ calmodulin-dependent protein kinase II (CaMKII), cAMP-dependent protein kinase A (PKA) and protein kinase C (PKC) correlates with increased transmitter release (Capogna et al, 1995; Hilfiker \& Augustine, 1999). These kinases control not only protein interactions within the release machinery but also the availability of free SNARE proteins that will form the functional fusion complex to facilitate exocytosis. They appear to be also involved in modulation of presynaptic plasticity by regulating the refilling of the RRP of SVs (Stevens \& Sullivan, 1998; Pang et al, 2010; Leenders \& Sheng, 2005).

\subsubsection{CaMKII}

CaMKII is a multifunctional holoenzyme expressed in the hippocampus at both the presynapse and the postsynapse. CaMKII is encoded by four genes in mammals $-\alpha, \beta, \gamma$, and $\delta$ - which in total give rise to about 30 isoforms (Hudmon \& Schulman, 2002; Tombes et al, 2003). The $\alpha$ and $\beta$ isoforms are predominantly expressed in the brain, while $\gamma$ and $\delta$ are expressed in most tissues (Erondu \& Kennedy, 1985; Tobimatsu \& Fujisawa, 1989; Burgin et al, 1990; Brocke et al, 1995; Hudmon \& Schulman, 2002). Each gene encodes a protein composed of an Nterminal serine-threonine kinase domain, followed by a regulatory region with an autoinhibitory sequence and a calmodulin (CaM)-binding site, and a C-terminal association or oligomerization domain responsible for assembly of subunits into large ring-shaped oligomers (Gaertner et al, 2004; Hudmon \& Schulman, 2002) (Figure 1.6A). The structure of the functional CaMKII enzyme is dodecameric, made up of two stacked hexameric rings (Chao et al, 2011) (Figure 1.6B-C). In basal $\mathrm{Ca}^{2+}$ concentrations, the kinase remains in an autoinhibitory state, with the catalytic domain sterically blocked by the regulatory domain that acts as a pseudosubstrate, preventing binding of substrates. CaMKII is activated by $\mathrm{Ca}^{2+} /$ calmodulin $(\mathrm{CaM})$ binding to the CaM-binding site of the regulatory domain, leading to a conformational change which exposes the catalytic domain, allowing the kinase to 
phosphorylate itself and other substrates (Rosenberg et al, 2005). Auto-phosphorylation renders the kinase $\mathrm{Ca}^{2+}$ - and CaM-independent, resulting in a sustained activation (Malenka, 2003).

Synapsin I (Llinás et al, 1985; Greengard et al, 1993; Ryan et al, 1996), synaptotagmin-1 (Popoli, 1993), syntaxin, SNAP-25, and VAMP2 (Nielander et al, 1995; Hirling \& Scheller, 1996; Turner et al, 1999) are substrates of CaMKII.

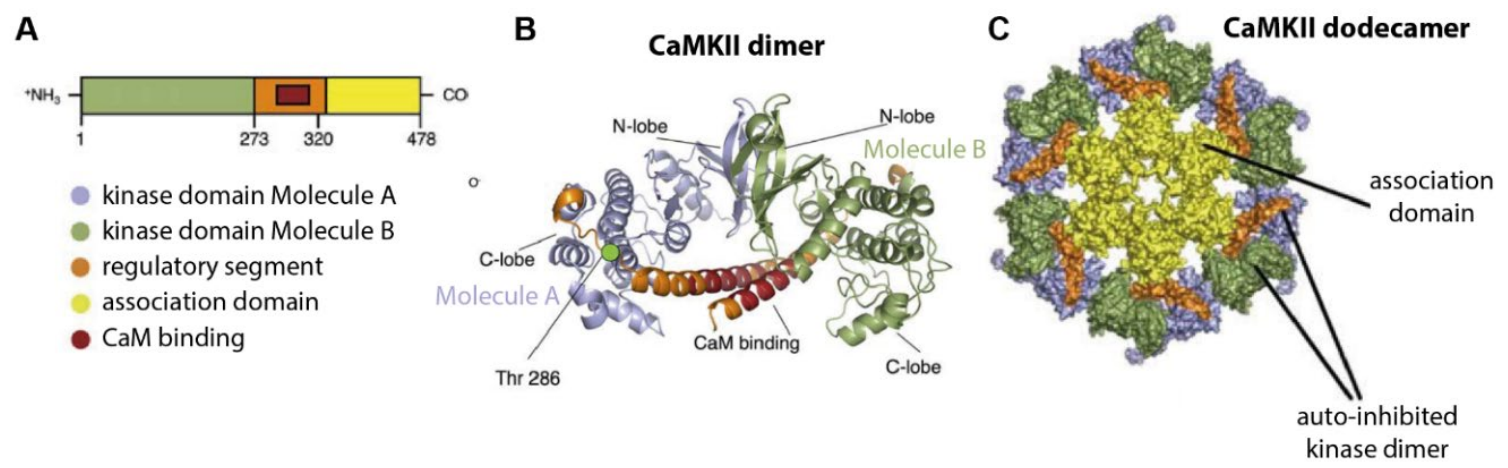

Figure 1.6. CaMKII domain organization and structure.

A. Schematic domain organization of CaMKII. B. View of the CaMKII dimer. C. Organization of CaMKII in a dodecamer structure (12 subunits). (A-C) Molecule A in purple and molecule B in green. $\mathrm{Ca}^{2+} / \mathrm{CaM}$ binding region in red and the rest of the regulatory segment in orange. Adapted from Rosenberg et al, 2005.

\subsubsection{PKC}

PKC was one of the first kinases to be identified (Inoue et al, 1977). In mammals the PKC family members are divided structurally and functionally in distinct groups according to their regulatory domains. Structurally they generally comprise a phospholipid-binding and diacylglycerol (DAG)/phorbol ester-binding $\mathrm{C}_{1}$ domain, followed by a calcium-binding $\mathrm{C}_{2}$ domain and a C-terminal kinase moiety - conventional or classical PKCs (cPKCs). Structural and functional variations gave rise to new classes of PKCs: novel (nPKC), atypical (aPKC) and PKN-related kinases (PKN) (reviewed in Rosse et al, 2010; Callender \& Newton, 2017) (Figure 1.7A). Of interest, cPKCs are activated by combined binding of DAG and phospholipid-binding to the $\mathrm{C}_{1}$ domain and $\mathrm{Ca}^{2+}$-dependent phospholipid-binding to the $\mathrm{C}_{2}$ domain. nPKCs are activated by DAG and phospholipids but do not respond to $\mathrm{Ca}^{2+}$, and aPKCs do not respond to DAG or $\mathrm{Ca}^{2+}$ but instead are allosterically activated by their PB1 (Phox and Bem1) domain with the PAR6-CDC42 complex involved in cell polarity. PKN are yet another variation where the PB1 regulatory domain was replaced by the homology region 1 (HR1) motif, which is activated by the GTPases Rho and Rac (Figure 1.7A). 
cPKCs are the most abundant and can be activated by phosphatidylserine (PS) and DAG in a $\mathrm{Ca}^{2+}$-dependent manner but also by DAG-mimetics phorbol esters (Castagna et al, 1982; Nishizuka, 1984). cPKC's activation occurs in a similar fashion to CaMKII's. A pseudosubstrate sequence in the regulatory domain blocks the substrate-binding site in the catalytic domain keeping the kinase in an inactive autoinhibited form that, in this specific case, targets the enzyme to the cytosol (Parker \& Murray-Rust, 2004; Newton, 2010; Gould et al, 2011; Antal et al, 2014, 2015). cPKC is activated by increases in the concentration of DAG and $\mathrm{Ca}^{2+}$ and subsequent binding of $\mathrm{Ca}^{2+}$ to the $\mathrm{C}_{2}$ domain, leading to an increased affinity of cPKC to phospholipids, which in turn results in its recruitment to the membrane (Verdaguer et al, 1999; Sánchez-Bautista et al, 2006; Evans et al, 2006). Once at the membrane cPKC binds to $\mathrm{DAG}$ via its $\mathrm{C}_{1}$ domain, yielding an open and active $\mathrm{PKC}$ form that will phosphorylate target substrates (Kraft et al, 1982; Sakai et al, 1997) (Figure 1.7B).

A

\begin{tabular}{|c|c|}
\hline Activators & Isoform \\
\hline Diacylglycerol, lipid and $\mathrm{Ca}^{2+}$ & $-\mathrm{cPKC}(\mathrm{PKC} \alpha, \mathrm{PKC} \beta, \mathrm{PKC} \gamma)$ \\
\hline Diacylglycerol, lipid & $-\mathrm{nPKC}(\mathrm{PKC} \delta, \mathrm{PKC} \varepsilon, \mathrm{PKC} \eta, \mathrm{PKC} \theta)$ \\
\hline PAR6-CDC42 & $-\mathrm{aPKC}(\mathrm{PKC} \zeta, \mathrm{PKCl} / \lambda)$ \\
\hline Rac and Rho & PKN (PKN1, PKN2, PKN3) \\
\hline
\end{tabular}
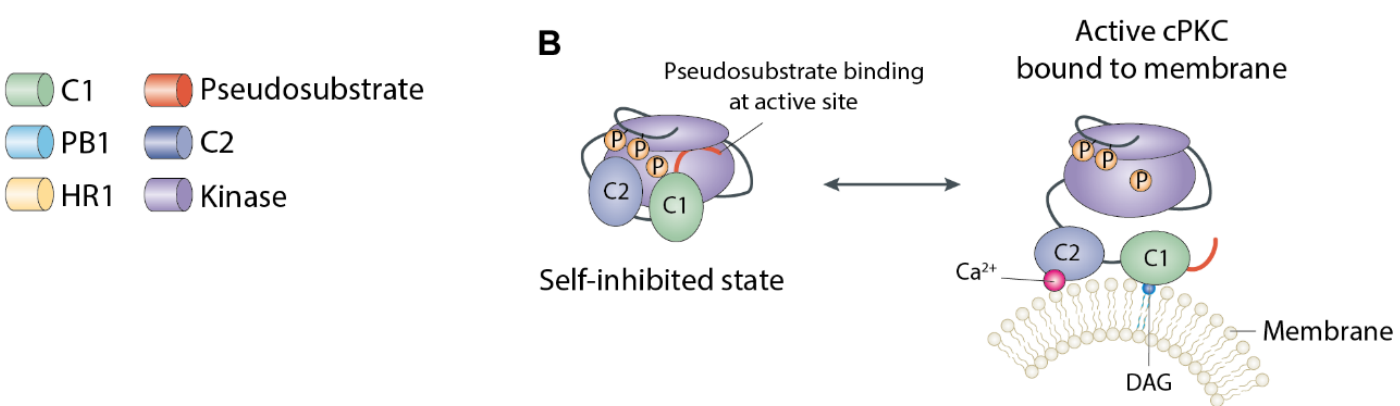

Figure 1.7. PKC kinase structure and families.

A. Schematic domain organization of the mammalian PKC family, which can be divided into four structurally and functionally distinct subgroups according to their regulatory domains: classical (cPKC), novel (nPKC), atypical (aPKC) and PKC-related kinases (PKN). B. A pseudosubstrate in its regulatory domains keeps PKC in a self-inhibited state through binding to the substrate-binding pocket in the kinase domain. Self-inhibition is relieved by different activators (indicated in A) depending on the subgroup. The regulatory domain is recruited to the plasma membrane and the catalytic domain of PKC is free to phosphorylate target substrates. Adapted from Rosse et al, 2010.

Crucial for multiple forms of presynaptic plasticity, the DAG/PKC pathway is one of the most potent pathways at the presynapse, with its activation leading to $50-100 \%$ potentiation of spontaneous and action potential-induced release (Malenka et al, 1986; Shapira et al, 1987; Lou et al, 2005). PKC was reported to phosphorylate several presynaptic proteins, e.g. SNAP25 (Shimazaki et al, 1996; Kataoka et al, 2000; Nagy et al, 2002; Genoud et al, 2001), 
Munc18-1 (Wierda et al, 2007; Barclay et al, 2003; Genç et al, 2014; Cijsouw et al, 2014) and Syt1 (Jong et al, 2016). For instance, in the case of Syt 1 - the $\mathrm{Ca}^{2+}$ sensor in conventional synapses - Jong et al proposed that its PKC-dependent phosphorylation enhances synaptic strength to action potential bursts. Mechanistically, phosphorylated Syt 1 seems to act in a cooperative way with phosphorylated Munc18-1 (PKC substrate) and Munc13-1 (DAG substrate but not PKC substrate; Rhee et al, 2002) to control a post-priming step of exocytosis.

In addition to phosphorylating exocytic proteins and thus facilitating neurotransmitter release from presynaptic terminals, PKC's central role in vesicular transport pathways has become evident, particularly in regulating membrane trafficking events and endocytosis (reviewed in Alvi et al, 2007). For instance, phosphorylation of synaptotagmin IX by PKC targets this protein to endocytic compartments (Haberman et al, 2005).

\subsubsection{CaMKII and PKC at inner hair cell ribbon synapses}

At IHC ribbon synapses, regulation of presynaptic activity via phosphorylation of the presynaptic release machinery is a field yet to be explored. To date, only otoferlin has been shown to be subject of such regulation, being phosphorylated by CaMKII $\delta$ (Meese et al, 2017 and this thesis). The phosphorylation of otoferlin by CaMKII $\delta$ changes the affinity of otoferlin's $\mathrm{C}_{2}$ domains to $\mathrm{Ca}^{2+}$, e.g. phosphorylation of the $\mathrm{C}_{2} \mathrm{~F}$ domain renders it $\mathrm{Ca}^{2+}$ insensitive under physiological conditions. This type of molecular modification might affect the kinetics of exocytosis, endocytosis and vesicle replenishment at IHC synapses.

\subsection{Scope of the project}

The large multi- $\mathrm{C}_{2}$ domain protein otoferlin is essential for hearing and fast $\mathrm{Ca}^{2+}$-triggered transmitter release from auditory IHCs (Roux et al, 2006; Pangršič et al, 2012), and it is involved in several steps of the synaptic vesicle cycle. While some progress has been made in understanding its role in IHC synaptic transmission, mechanisms regulating its function were not studied to date. In conventional synapses, second messenger-activated protein kinases expressed in presynaptic nerve terminals phosphorylate presynaptic proteins thereby regulating i) presynaptic plasticity by controlling the refilling of the RRP of vesicles, ii) protein interactions within the release apparatus and iii) endocytosis and trafficking events (Südhof, 1995; Turner et al, 1999; Haberman et al, 2005; Jong et al, 2016). It is unknown if protein kinases also regulate presynaptic transmission in IHC ribbon synapses, and to assess this was the main goal of this thesis.

The first part of my thesis focused on assessing the role of CaMKII in IHC synaptic transmission. This was an ongoing project started before the beginning of my doctoral studies, 
and I helped guiding it to completion during the course of my thesis. Before I joined the project in vitro and mass spectrometry studies showed that otoferlin and CaMKII interact, that otoferlin is phosphorylated by CaMKII, and that this phosphorylation affects the affinity of otoferlin's $\mathrm{C}_{2}$ domains to $\mathrm{Ca}^{2+}$. Additionally, it was found that phosphorylation of otoferlin and/or otoferlin-bearing protein complexes is enhanced upon hair cell depolarization in rat IHCs. However, the question remained on which CaMKIIs were mainly expressed in rodent IHCs. To answer this question, I performed immunohistochemistry on dissected organs of Corti and real-time PCR experiments on a few isolated IHC of C57BL/6J mice. Using in situ proximity ligation assays (PLAs), I additionally investigated if otoferlin and CaMKII are in close proximity and if phosphorylation was enhanced upon IHC stimulation also in murine IHCs. The obtained results were partially published in Meese et al (2017).

Another goal of this thesis was to assess the potential role of other kinases in IHC synaptic function. I screened for the expression of other kinases in murine IHCs, I assessed if they interact with and phosphorylate otoferlin, and evaluated potential changes upon IHC stimulation. For this purpose, I combined immunohistochemistry and PLA assays on explanted organs of Corti, confocal microscopy, pull-downs, co-immunoprecipitations and in vitro assays. 
26 | General Introduction 
Chapter 2: CaMKIII is expressed in the organ of Corti and regulates otoferlin's activity during strong inner hair cell stimulation 



\section{Published article}

Meese S, Cepeda AP, Gahlen F, Adams CM, Ficner R, Ricci AJ, Heller S, Reisinger E, Herget M (2017) Activity-dependent phosphorylation by CaMKII $\delta$ alters the $\mathrm{Ca}^{2+}$ affinity of the multi-C ${ }_{2}$-domain protein otoferlin. Front Synaptic Neurosci 9: 13.

(see original publication in Appendix)

\subsection{Synopsis}

Otoferlin is essential for fast $\mathrm{Ca}^{2+}$-triggered transmitter release from auditory IHCs and it has been shown to be involved in several steps of the synaptic vesicle cycle including vesicle fusion, vesicle reformation, vesicle replenishment, and active zone clearance via coupling of exo- and endocytosis (Roux et al, 2006; Pangrsic et al, 2010; Duncker et al, 2013; Jung et al, 2015a; Strenzke et al, 2016; Michalski et al, 2017). While great progress has been made in understanding the essential role of otoferlin in IHC synaptic function, mechanisms regulating its activity have not been studied to date.

My collaborators and I showed for the first time that similarly to what happens in conventional synapses, synaptic activity in IHC synapses is regulated by phosphorylation of presynaptic proteins, in this case otoferlin. Combining immunohistochemistry, in situ proximity ligation assays (PLAs), confocal microscopy, real-time PCR, mutagenesis, microscale thermophoresis (MST), pull-downs, co-immunoprecipitation (co-IP), in vitro assays and mass spectrometry approaches, we found that $\mathrm{Ca}^{2+} /$ calmodulin-dependent serine/threonine kinase delta (CaMKIIS) phosphorylates otoferlin and regulates its activity in rodent IHCs. Firstly, Dr. Meike Herget identified CaMKII $\delta$ as a binding partner of otoferlin via pull-down assays with chicken utricle lysates and co-IPs with heterologously expressed proteins in HEK293 cells. Dr. Meike Herget confirmed the expression of CaMKII $\delta$ in rat IHCs via immunohistochemistry. I then proved that CaMKII $\delta$ is the main CaMKII expressed in rodent IHCs (with a minor contribution of CaMKII $\gamma$ ) via real-time PCR and immunohistochemistry on C57BL/6J mice. A PLA revealed close proximity between otoferlin and CaMKII ( $\alpha-\delta$ and $\delta$ ) in rat (performed by Dr. Meike Herget) and mouse (performed by me) IHCs, suggesting a probable interaction also in vivo. Dr. Sandra Meese and the Mass Spectrometry Unit of Stanford University (Stanford, CA, USA) identified ten phosphorylation sites in otoferlin via mass spectrometry 
following an in vitro assay, five of which are located within otoferlin's $\mathrm{C}_{2}$-domains. Exchange of these phosphorylated serine/threonine residues by phosphomimetic aspartates led to a 10fold reduction of the $\mathrm{C}_{2} \mathrm{~F}$ domain affinity to $\mathrm{Ca}^{2+}$ and increased the affinity of the $\mathrm{C}_{2} \mathrm{C}$ domain to $\mathrm{Ca}^{2+}$. Additionally, Dr. Meike Herget showed that phosphorylation of otoferlin and/or otoferlin-baring protein complexes is enhanced upon hair cell stimulation and is partially blocked by pharmacological inhibition of CaMKIII in rat IHCs. I found that in mouse IHCs otoferlin and CaMKII are also in close proximity and phosphorylation is enhanced upon stimulation. Our data suggests that the functions of otoferlin might be regulated by CaMKII $\delta$ during strong IHC depolarization.

In this chapter, I present original data partially published in Meese et al (2017), corresponding to the investigation of the expression and localization of different CaMKIIs in mouse IHCs via immunohistochemistry and real-time PCR (chapters 2.3.1 and 2.3.2, Figures 1 and 2 in Meese et al (2017)). Only studies on the proximity between otoferlin and CaMKII and on the activitydependent phosphorylation of otoferlin protein complexes in rat IHCs, performed by Dr. Meike Herget, were included in Meese et al (2017). I further validated and confirmed these findings in mouse IHCs and present them here in chapters 2.4.1 and 2.4.2.

\subsection{Own contribution}

\section{Contribution to Meese et al (2017):}

- Figure 1: Immunohistochemistry (stainings of CaMKII $\alpha-\delta$ in combination with otoferlin and with pre- and postsynaptic markers in organs of Corti of C57BL/6J mice), confocal microscopy, data analysis (using ImageJ), figure preparation (identical to Figure 2.1 in chapter 2.3.1).

- Figure 2: Real-time PCR experiments (primer design, real-time PCR experiments, gel electrophoresis, data analysis; cells were collected by PD Dr. Ellen Reisinger), figure preparation (identical to Figure 2.2 in chapter 2.3.1).

- Writing, editing and revision of the manuscript together with all authors. See also "Author Contributions" section in Meese et al (2017) (see original publication in Appendix).

\section{Additional data not published in Meese et al (2017):}

- Figure 2.3: Immunohistochemistry (stainings of CaMKII $\alpha-\delta$ in combination with parvalbumin) and proximity ligation assays (combinations: otoferlin with CaMKII $\alpha-\delta$ 
and otoferlin with CaMKII $\delta$ ) in organs of Corti of C57BL/6J mice, confocal microscopy, data analysis (using ImageJ), figure preparation.

- Figure 2.4: Immunohistochemistry (stainings of phosphoserine residues in combination with otoferlin) and proximity ligation assay between otoferlin and phosphoserine residues in organs of Corti of C57BL/6J mice, confocal microscopy, data analysis (using ImageJ), figure preparation.

All experiments and subsequent data analysis were performed as described in the "Materials and Methods" section of Meese et al (2017).

\subsection{Published results}

\subsubsection{Expression and cellular distribution of different CaMKIIs in the organ of Corti}

This section is based on Meese et al (2017), with emphasis on the work I was significantly involved in.

With the goal of studying the subcellular localization of the different CaMKIIs in mammalian

IHCs, I started by performing immunohistochemistry on acutely explanted organs of Corti of P14 wild-type C57BL/6J (henceforth, B6) mice (Figure 2.1). Used antibodies and detailed methods are described in the "Materials and Methods" section of Meese et al (2017).

No CaMKII $\alpha$ expression was detected in IHCs (Figure 2.1A). CaMKII $\beta$ (Figure 2.1B), $\gamma$ (Figure 2.1C) and $\delta$ (Figure 2.1D) were found in regions outside the IHCs, possibly in efferent and/or afferent synaptic boutons. Additionally, CaMKII $\delta$ immunofluorescence was detected in the cytoplasm of IHCs but also in regions near the active zones (Figure 2.1E) where it colocalized with the postsynaptic protein PSD95 (Figure 2.1F). Although an apparent colocalization with the postsynaptic protein PSD95 potentially indicates a postsynaptic localization for CaMKII $\delta$, the localization of the protein at the presynapse cannot be excluded. The small volume of the synaptic cleft made it impossible to narrow down the synaptic localization of CaMKII $\delta$ via conventional confocal microscopy. 


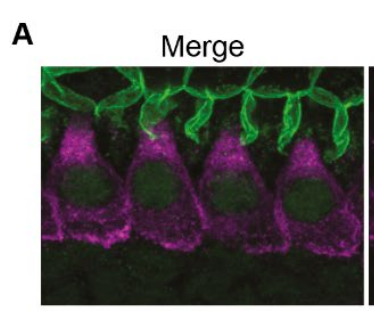

B

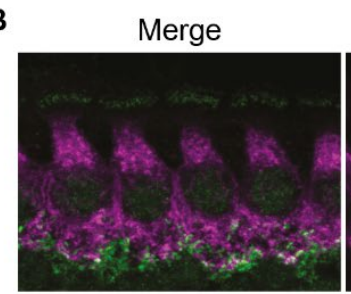

C

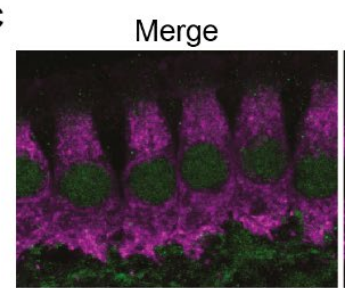

D

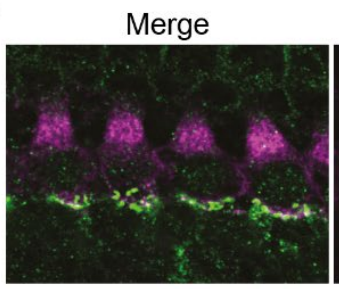

Otoferlin

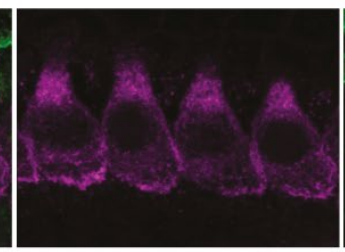

Otoferlin

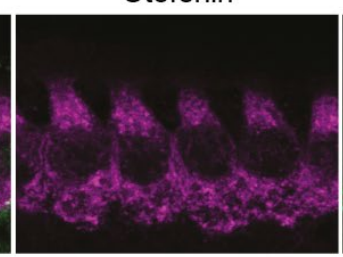

Otoferlin

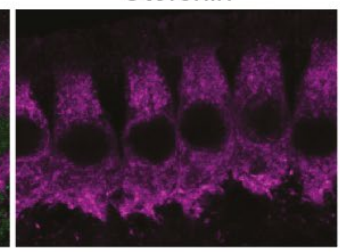

Otoferlin

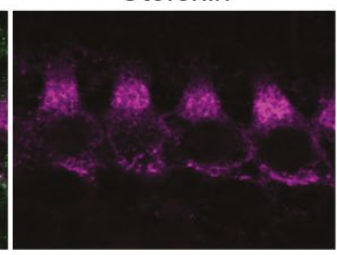

CaMKII $\alpha$

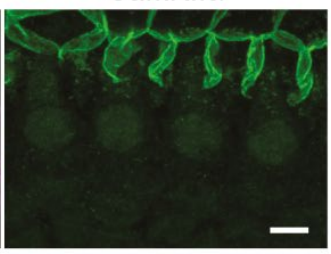

CaMKII $\beta$

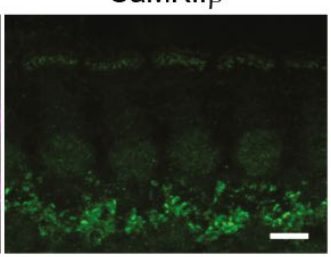

CaMKIly

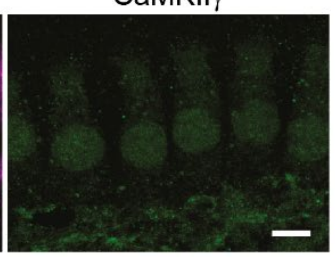

CaMKII

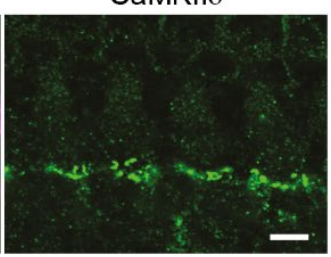

E

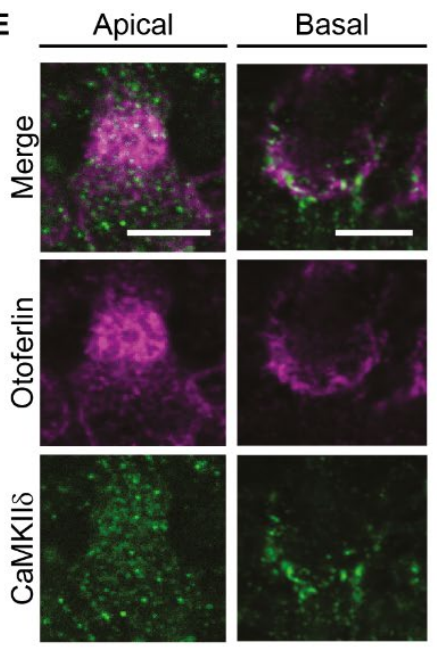

F

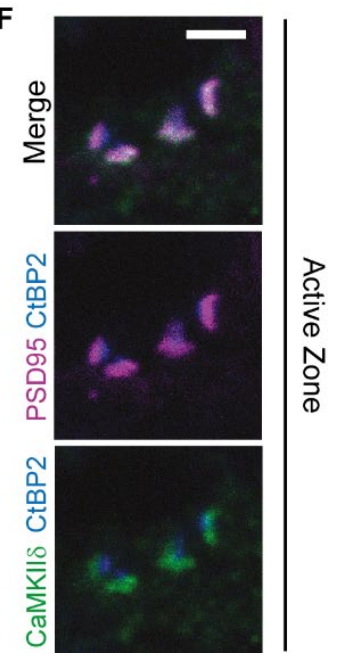

Figure 2.1. Localization of the different CaMKIIs in the murine organ of Corti.

A-D High magnification views of representative wild-type B6 P14 mouse IHCs immunolabeled for CaMKII $\alpha$ (A), CaMKII $\beta$ (B), CaMKII $\gamma$ (C) and CaMKII (D) (green) and otoferlin (magenta).

E Higher magnification views of a representative IHC displayed in D, showing apical and basal regions for better visualization of subcellular localization of CaMKIIS.

F Higher magnification views of the synaptic area of a representative IHC, immunolabeled against CaMKII (green), the ribbon marker CtBP2 (blue) and the postsynaptic protein PSD95 (magenta).

Colocalization between CaMKII $\delta$ and PSD95 is displayed in white in the merged image (top panel).

Single confocal optical sections. Scale bars: $5 \mu \mathrm{m}$ (A-E), $2 \mu \mathrm{m}$ (F). IHC, inner hair cell. Based on Figure 1 of Meese et al, 2017.

\subsubsection{CaMKII $\delta$ is the predominant CaMKII in rodent IHCs}

This section is based on Meese et al (2017), with emphasis on the work I was significantly involved in.

To investigate the presence of CaMKII transcripts in the organ of Corti, and more specifically in IHCs, a few IHCs were isolated with the aid of a patch-clamp setup. 3 to 5 IHCs per 
biological replicate were collected (Samples 1 to 3, Figure 2.2). Negative bath controls (small volume of bath solution in close proximity to the IHC row prior to and directly after extraction of the IHC's cytoplasm) were also collected. I then designed specific primers for each of the four CaMKII genes and performed real-time PCR experiments with a few isolated IHCs. For this, RNA from IHCs was reverse transcribed into complementary DNA (cDNA). Mouse brain cDNA was used as positive control in PCR reactions with SYBR green (Figure 2.2.E). Only samples with positive TaqMan-PCR signals for both housekeeping genes, bassoon and TATA-binding protein, were considered for analysis. Detailed methods are described in the "Materials and Methods" section of Meese et al (2017). Note that IHCs were harvested by PD Dr. Ellen Reisinger. Primer design, PCR reactions and subsequent data analysis were performed by me.

In these experiments, there was no amplification of CaMKII $\alpha$ or CaMKII $\beta$ mRNA (Figure 2.2A-C). CaMKII $\delta$ transcripts were identified in three independent samples (Figure 2.2A-C) and CaMKII $\gamma$ transcripts in one of the samples only (Figure 2.2C). These results point to a major role of CaMKII $\delta$ in rodent IHCs, with a minor but supporting contribution of CaMKII $\gamma$.
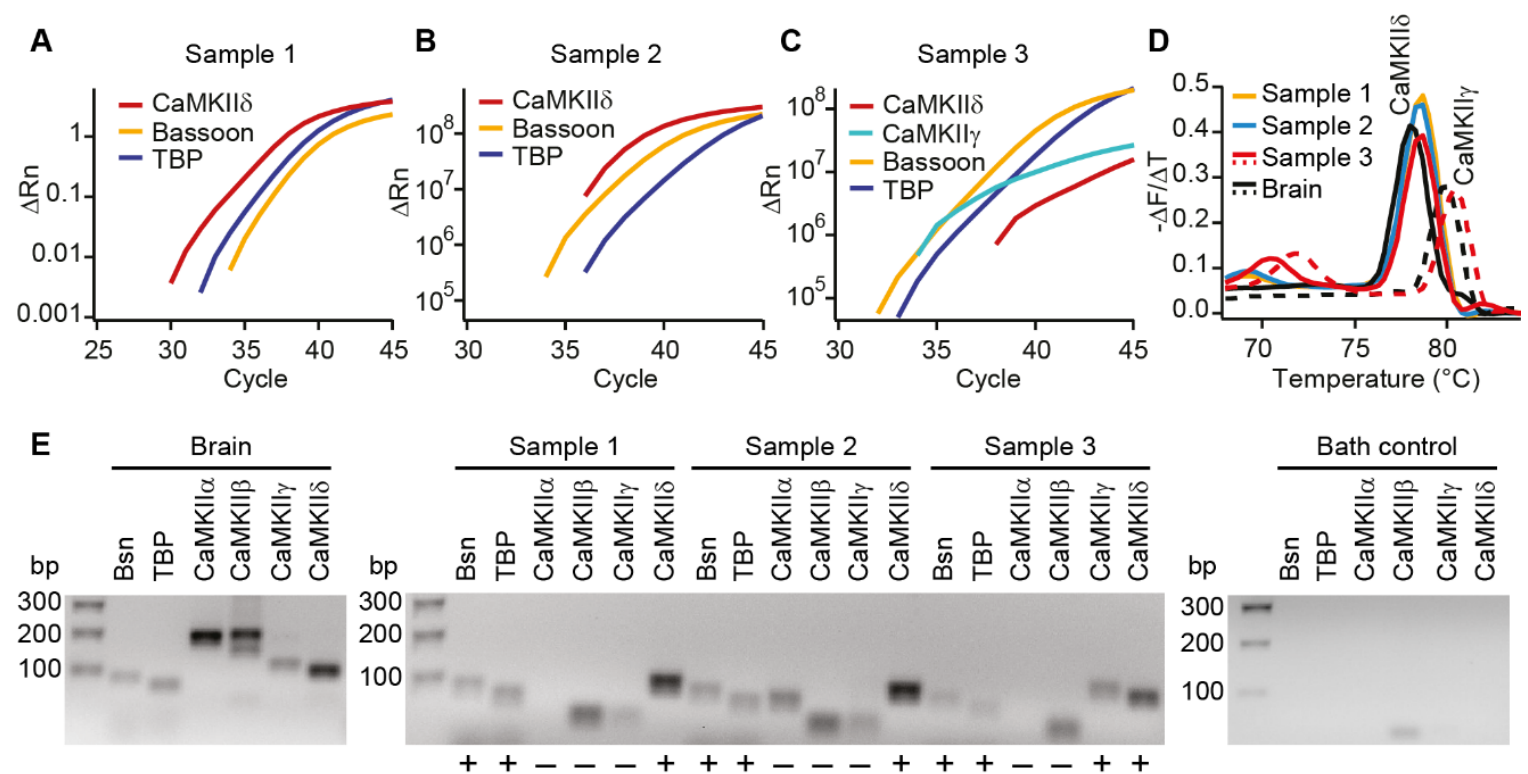

Figure 2.2. CaMKII $\delta$ transcripts are predominantly expressed in murine IHCs.

Real-time PCR analysis of CaMKII transcripts: CaMKII $\gamma$ and $\delta$ are expressed in IHCs of P14 wild-type B6 mice.

A-C Cytoplasm of 3-5 IHCs per sample analyzed by PCR for the mRNA expression of CaMKII $\alpha, \beta, \gamma$, and $\delta$ (SYBR green reactions). Primers specific for each of the four CaMKII genes were designed. TaqMan assays for bassoon (Bsn) and TATA-binding protein (TBP) were used as internal controls.

D Melting curve analysis for SYBR green assays of the three IHC cDNA samples and control brain cDNA samples.

E Amplicons from positive control experiments on brain cDNA (Brain), IHC samples (Samples 1 to 3) and one representative bath control, analyzed by agarose gel electrophoresis.

Based on Figure 2 of Meese et al, 2017. 


\subsection{Complementary studies}

\subsubsection{CaMKII interacts with otoferlin in murine IHCs}

Dr. Meike Herget first observed that CaMKII $\alpha-\delta$ and otoferlin are in close proximity in rat IHCs, and the distance between the two proteins decreases upon stimulation. For this, she used a proximity ligation assay (PLA) that she established for rat IHCs (Figure 4 in Meese et al (2017)). This assay allows in situ detection of proximity between two protein, with a signal being produced only if the two proteins are closer than $40 \mathrm{~nm}$ (Koos et al, 2014). I next investigated if otoferlin and CaMKII are in close proximity also in mouse IHCs.

A

Parvalbumin CaMKIIpan

Parvalbumin
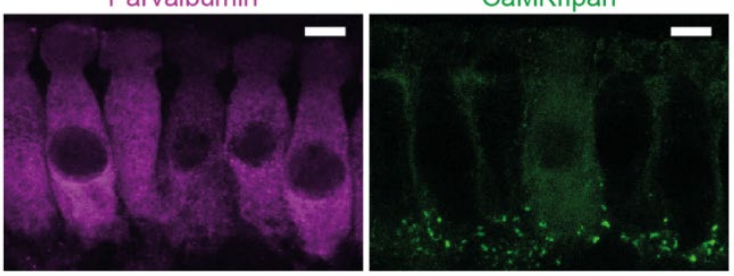

B

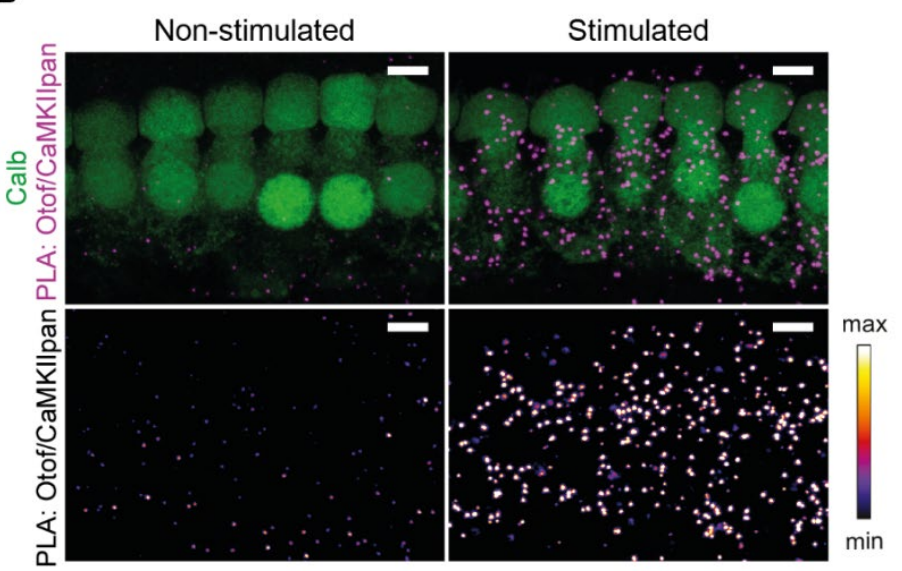

C

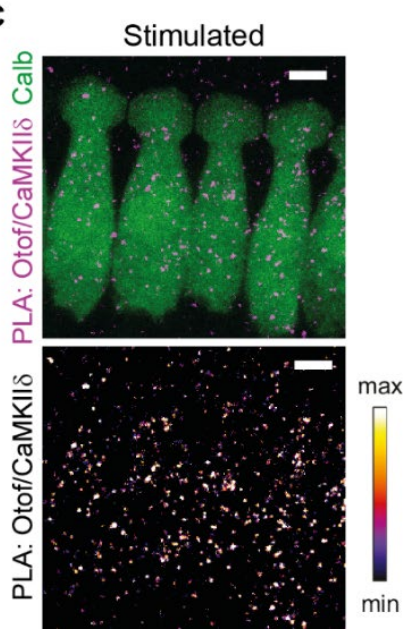

Figure 2.3. Activity-dependent interaction of otoferlin and CaMKII in mouse IHCs.

Proximity ligation assay reveals close proximity between otoferlin and CaMKII and the interaction is potentiated by high $\mathrm{K}^{+}$stimulation.

A High magnification view of representative wild-type B6 P15 IHCs immunolabeled for CaMKIIpan (green) with the antibody used for the PLA shown in (B). Parvalbumin (magenta) was used as IHC marker.

B High magnification views of representative PLAs for otoferlin and CaMKIIpan performed on wild-type B6 P14-P20 IHCs at rest and after strong stimulation with $40 \mathrm{mM} \mathrm{KCl}$ for 15 minutes at $37^{\circ} \mathrm{C}$.

C High magnification views of a representative PLA for otoferlin and CaMKII $\delta$ performed on wild-type B6 P14-P20 IHCs after strong stimulation with $40 \mathrm{mM} \mathrm{KCl}$ for 15 minutes at $37^{\circ} \mathrm{C}$.

CaMKIIpan refers to anti-CaMKII $\alpha-\delta$ antibody against the kinase domain, highly conserved among all CaMKII genes. In (A-C), maximum intensity projections of confocal optical sections. Scale bars: $5 \mu \mathrm{m}$. In (B, C), PLA channel is depicted with an intensity-coded lookup table with warmer colors representing higher pixel intensities; calbindin-D28k (green) was used as IHC marker. IHC, inner hair cell. Otof, otoferlin. Calb, calbindin-D28k. 
The PLA between otoferlin and CaMKII $\alpha-\delta$ (Figure 2.3B) performed in explanted organs of Corti of P14-20 mice in resting conditions resulted in hardly any fluorescent puncta distributed throughout the cytoplasm of the IHC. When the same PLA was done after a 15minute stimulation period with high $\mathrm{K}^{+}$(Figure 2.3B, right panel), there was an increase in PLA signal when compared to resting conditions (Figure 2.3B, left panel). The same happened for a PLA between otoferlin and CaMKIIS (Figure 2.3C). These data confirm the results obtained for rat IHCs reported in Meese et al (2017).

\subsubsection{Activity-dependent phosphorylation of otoferlin or otoferlin interaction partners in murine IHCs}

In Meese et al (2017) a PLA to find phosphoserine residues in close proximity to otoferlin was used to test whether otoferlin or proteins interacting with otoferlin are phosphorylated and to assess if this phosphorylation is activity-dependent in rat IHCs (Figure 10 in Meese et al (2017)). My aim was to confirm these findings in mouse IHCs. My results are in agreement with what was observed for rat IHCs. In resting conditions, the PLA for otoferlin and phosphoserine residues resulted in a few puncta across the cytoplasm of the IHCs pointing towards a certain degree of basal phosphorylation (Figure 2.4B, left panel). After a 15-minute high $\mathrm{K}^{+}$stimulation there was an increase in PLA signal intensity (Figure $2.4 \mathrm{~B}$, right panel) and the PLA puncta did not overlap but were found in close proximity to the synaptic ribbon, as observed via co-staining with the ribbon marker CtBP2 (Figure 2.4C-C'). Given the rather basal location of the PLA puncta, it is possible that important phosphorylation events might occur in regions close to the basolateral plasma membrane, where the active zones reside in these cells, or in endocytic compartments, both situated in close proximity to the synaptic ribbons (Duncker et al, 2013; Neef et al, 2014; Revelo et al, 2014; Jung et al, 2015a). In Meese et al (2017), it was additionally shown that the stimulation-induced increase in PLA signal is at least in part CaMKII-dependent since this effect could be partially blocked by the CaMKII inhibitor KN-93.

From these results we can infer that protein complexes of which otoferlin is part of seem to be phosphorylated upon strong hair cell depolarization. These phosphorylation events appear to occur in close proximity to the ribbons and seem to be promoted at least partially by CaMKII. 
A

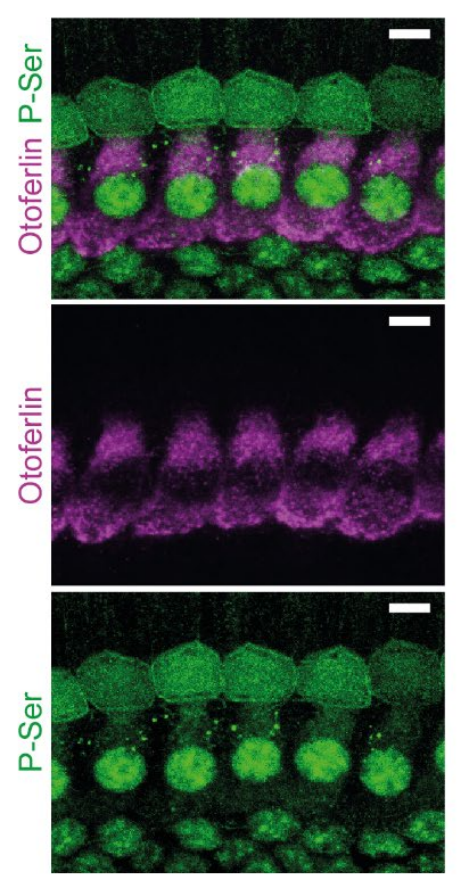

B
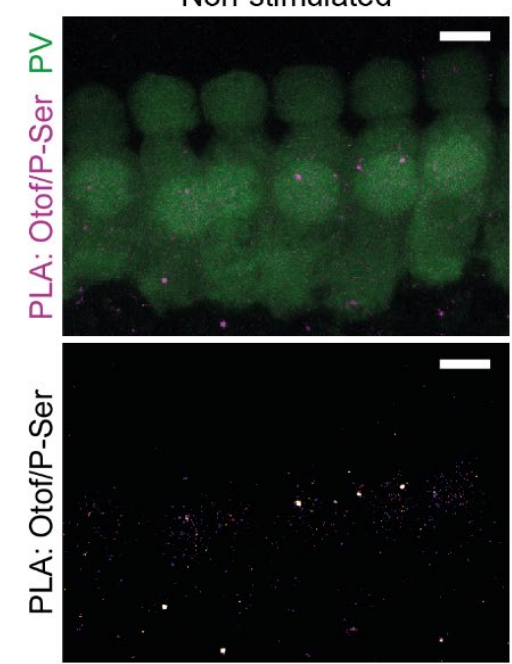

C

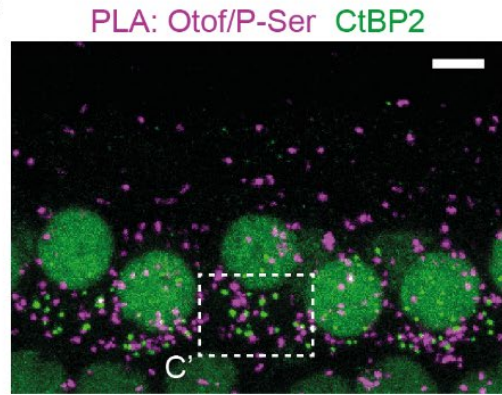

Stimulated

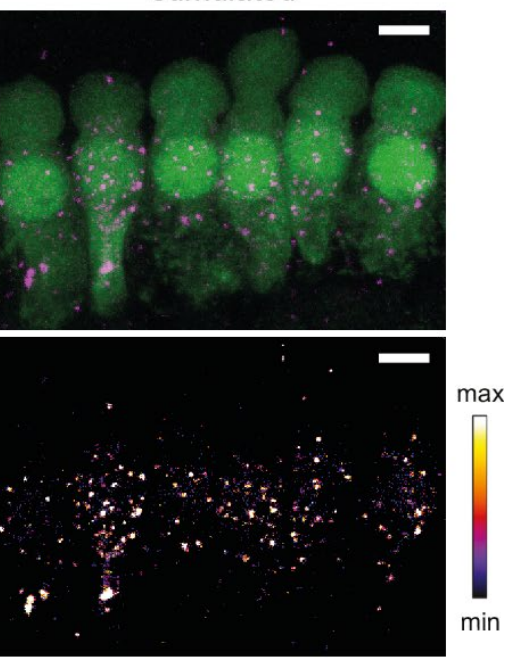

PLA: Otof/P-Ser CtBP2

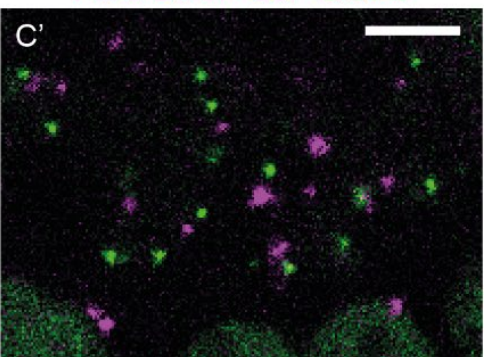

Figure 2.4. Phosphorylation of otoferlin complexes is strongly promoted by strong hair cell stimulation.

A High magnification view of representative wild-type B6 P14 IHCs immunolabeled for otoferlin (magenta) and phosphoserine (green), with the antibodies used for the PLAs shown in (B, C).

B High magnification views of representative PLAs for otoferlin and phosphoserine residues (magenta) performed on wild-type B6 P14-P20 IHCs at rest and after strong stimulation with $40 \mathrm{mM} \mathrm{KCl}$ for 15 minutes at $37^{\circ} \mathrm{C}$. Parvalbumin (green) was used as IHC marker. PLA channel (magenta) is also depicted separately with an intensity-coded lookup table with warmer colors representing higher pixel intensities (bottom panel).

C High magnification views of a representative PLA for otoferlin and phosphoserine residues (magenta) performed on wild-type B6 P14-P20 IHCs after strong stimulation with $40 \mathrm{mM} \mathrm{KCl}$ for 15 minutes at $37{ }^{\circ} \mathrm{C}$. Co-staining with the ribbon marker $\mathrm{CtBP} 2$ (green) indicates close proximity but no colocalization of phosphorylated otoferlin complexes with synaptic ribbons (C').

In (A-C), maximum intensity projections of confocal optical sections. In (C'), single confocal optical section. Scale bars: $5 \mu \mathrm{m}$. IHC, inner hair cell. Otof, otoferlin. P-Ser, phosphoserine. PV, parvalbumin. 
Chapter 3: PKC $\alpha$ promotes the interaction of otoferlin with calbindin and regulates endocytosis at auditory inner hair cell synapses 



\section{Published article}

Cepeda AP, Al-Moyed H, Lenz C, Urlaub H, Reisinger E (2019) PKC $\alpha$-dependent interaction of otoferlin and calbindin: evidence for regulation of endocytosis in inner hair cells. bioRxiv 779520; doi: https://doi.org/10.1101/779520.

\subsection{Synopsis}

From Chapter 2 and from Meese et al (2017) it becomes evident that otoferlin's function in rodent IHCs can be modulated by CaMKII. It is noteworthy that when a PLA was used to test whether otoferlin and/or proteins interacting with otoferlin are phosphorylated (PLA for otoferlin and phosphoserine residues) in an activity-dependent manner in rat IHCs, the PLA signal increased with high $\mathrm{K}^{+}$stimulation when compared to resting conditions, and this effect could be only partially blocked by the CaMKII inhibitor KN-93. This suggests that other kinases can potentially be involved in regulating IHC synaptic function. I then screened for the presence of other kinases and found protein kinase $\mathrm{C} \alpha(\mathrm{PKC} \alpha)$ to be highly expressed in murine IHCs. IHC depolarization via high $\mathrm{K}^{+}$stimulation led to the activation and targeting of PKC $\alpha$ to endocytic compartments where it colocalized with otoferlin. Upon strong IHC stimulation the PLA signal for the pair PKC $\alpha$-otoferlin increased over resting conditions, confirming close proximity of the two proteins during strong stimulation. A pull-down assay with partially purified otoferlin and an organ of Corti homogenate and coimmunoprecipitation assays with heterologously overexpressed proteins confirmed that otoferlin and PKC $\alpha$ can interact. An in vitro assay with co-incubated otoferlin and recombinant PKC $\alpha$ followed by subsequent mass spectrometry analysis confirmed the interaction and revealed that PKC $\alpha$ phosphorylated otoferlin at five serine residues. The PLA signal for otoferlin and phosphoserine residues increased over resting conditions upon strong IHC stimulation and after treatment with PMA (phorbol 12-myristate 13-acetate; a PKC activator). The stimulation-dependent increase in PLA signal was blocked to a large extend by the PKC inhibitor BIM I (bisindolylmaleimide I, a PKC inhibitor), but was fully blocked by co-treatment with $\mathrm{KN}-93$ and BIM I. This suggests that the activity-dependent phosphorylation of otoferlin and/or its interactors in mouse IHCs relies on the combined action of PKC and CaMKII. Additionally, the previously reported interaction between otoferlin and myosin VI appears to be PKC-dependent: i) the PLA signal for otoferlin-myosin VI increased after PKC activation either upon high $\mathrm{K}^{+}$stimulation or pharmacological 
activation with PMA; ii) the stimulation-dependent increase in PLA signal was fully blocked by the PKC inhibitor BIM I. Furthermore, I show that upon strong IHC stimulation and pharmacological PKC activation, otoferlin interacts with the EF-hand protein calbindinD28k, while PKC $\alpha$ and calbindin-D28k seem not to interact directly. The physical association of otoferlin and calbindin-D28k was further confirmed via pull-down assays.

In summary, by combining co-immunoprecipitation and pull-down assays, PLAs, immunohistochemistry, confocal microscopy, and mass spectrometry approaches, I could show that otoferlin is phosphorylated by PKC $\alpha$ in an activity-dependent manner, and the interaction of the two proteins was characterized by short-living accumulations in common large endocytic compartments. Moreover, otoferlin interacts with myosin VI and calbindinD28k in an activity-dependent and PKC $\alpha$-dependent manner. The PKC-dependent interaction of otoferlin with calbindin-D28k potentially regulates different modes of membrane internalization and might control the dynamics of the SV cycle in IHCs. This cooperative mechanism might constitute a molecular switch that provides the basis for the fast and efficient vesicle recycling characteristic of IHC ribbon synapses.

The contribution of other calcium buffer proteins like parvalbumin and calretinin in exocytic and/or endocytic events was also explored. Positive PLAs for otoferlin-parvalbumin and otoferlin-calretinin indicate close proximity of the proteins, and parvalbumin and calretinin immunofluorescence levels were altered in different otoferlin mouse models.

Altogether, my data suggests that otoferlin might act in collaboration with other $\mathrm{Ca}^{2+}$-binding proteins (like $\mathrm{PKC} \alpha$, calbindin, parvalbumin and calretinin) rather than being an exclusive $\mathrm{Ca}^{2+}$ sensor for exocytic and/or endocytic processes in IHCs.

\subsection{Own contribution}

\section{Contribution to Cepeda et al (2019):}

- Figure 3.1: Immunohistochemistry, confocal microscopy, data analysis, statistics, illustration, figure preparation.

- Figure 3.2: Immunohistochemistry, confocal microscopy, data analysis, illustration, figure preparation.

- Figure 3.3: Proximity ligation assays, confocal microscopy, cloning, heterologous protein expression in HEK293T cells, co-immunoprecipitation and pull-down assays, western blotting, in vitro phosphorylation assay, data analysis, statistics, illustrations, figure preparation. 
- Figure 3.4: Immunohistochemistry, confocal microscopy, data analysis, statistics, figure preparation.

- Figure 3.5: Proximity ligation assays, confocal microscopy, data analysis, statistics, figure preparation.

- Figure 3.6: Immunohistochemistry, proximity ligation assays, confocal microscopy, data analysis, statistics, figure preparation.

- Figure 3.7 (A-D, F-H): Immunohistochemistry, proximity ligation assays, confocal microscopy, data analysis, statistics, pull-down assays, western blotting, illustration and figure preparation.

- Figure EV1: Immunohistochemistry, confocal microscopy, data analysis, figure preparation.

- Figure EV2: Immunohistochemistry, confocal microscopy, data analysis, figure preparation.

- Figure EV3A: Immunohistochemistry, confocal microscopy, data analysis and figure preparation.

- Figure EV4: Proximity ligation assays, confocal microscopy, data analysis, statistics, figure preparation.

- Appendix Figure S1: Immunohistochemistry, proximity ligation assays, confocal microscopy, data analysis, figure preparation.

- Appendix Figure S2: Proximity ligation assays, confocal microscopy, data analysis, figure preparation.

- Appendix Figure S10: Sequence alignment and mapping of phosphorylation sites in the otoferlin sequence, figure preparation.

- Appendix Figure S11: Sequence alignment and mapping of phosphorylation sites in the otoferlin sequence from different species, figure preparation.

- Appendix Figure S12: Venn diagram of PKC phosphorylation sites prediction, figure preparation.

- Appendix Table S1. Statistical analysis summary.

- Appendix Table S2: PKC phosphorylation sites prediction.

Mass spectrometry analysis of phosphorylation sites on otoferlin depicted in Figure 3.3 and Appendix Figures S3-S9 of Cepeda et al (2019) were performed by Dr. Christof Lenz (Core Facility Proteomics, Institute of Clinical Chemistry, University Medical Center Göttingen and Bioanalytical Mass Spectrometry Group, Max Planck Institute for Biophysical Chemistry, Göttingen, Germany) with the technical assistance of Lisa Neuenroth as referred in "Author Contributions" section in Cepeda et al (2019). Otoferlin rescue experiments, confocal microscopy, data analysis, statistics and figure preparation in Figures Figure 3.7E and Figure EV3B were performed by Dr. Hanan Al-Moyed (InnerEarLab, University Medical Center 
Göttingen, Germany). Gerhard Hoch (Institute for Auditory Neuroscience and InnerEarLab, University Medical Center Göttingen) developed the custom-written Matlab (MathWorks) routine integrated into Imaris 7.6.5 (Bitplane Scientific Software) for immunofluorescence level quantifications (see "Materials and Methods" section in Strenzke et al, 2016) and Dr. Hanan Al-Moyed further helped improving it during the course of her Ph.D. thesis.

All experiments and subsequent data analysis, statistical analysis and figure preparation were carried out during the course of this thesis as described in the "Materials and Methods" section of Cepeda et al (2019). The manuscript for this publication was written, edited and revised together with all authors (see "Author Contributions" section in Cepeda et al (2019)).

\section{Additional data not included in Cepeda et al (2019):}

- Figure 3.8: Proximity ligation assays, confocal microscopy, data analysis, figure preparation.

- Figures 3.9 and 3.10: Immunohistochemistry, confocal microscopy, data analysis, statistics, figure preparation.

\subsection{Manuscript}

Title: PKC $\alpha$-dependent interaction of otoferlin and calbindin: evidence for regulation of endocytosis in inner hair cells

\section{Running title: PKC $\alpha$ promotes otoferlin-calbindin binding}

Authors: Andreia P. Cepeda ${ }^{1,2,3}$, Hanan Al-Moyed ${ }^{1,3}$, Christof Lenz ${ }^{4,5}$, Henning Urlaubb ${ }^{2,4,5}$, Ellen Reisinger ${ }^{1,2,6, *}$

${ }^{1}$ Molecular Biology of Cochlear Neurotransmission Group, Department of Otorhinolaryngology, University Medical Center Göttingen, Göttingen, Germany

${ }^{2}$ Collaborative Research Center 889, University of Göttingen, Göttingen, Germany

${ }^{3}$ Göttingen Graduate Center for Neurosciences, Biophysics, and Molecular Biosciences, University of Göttingen, Göttingen, Germany

${ }^{4}$ Core Facility Proteomics, Institute of Clinical Chemistry, University Medical Center Göttingen, Göttingen, Germany

${ }^{5}$ Bioanalytical Mass Spectrometry Group, Max Planck Institute for Biophysical Chemistry, Göttingen, Germany ${ }^{6}$ Present address: Gene Therapy for Hearing Impairment and Deafness, Department of Otorhinolaryngology, University Hospital Tübingen, Germany

"Corresponding author. E-mail: ellen.reisinger@med.uni-goettingen.de 


\subsubsection{Synopsis and Graphical Abstract}

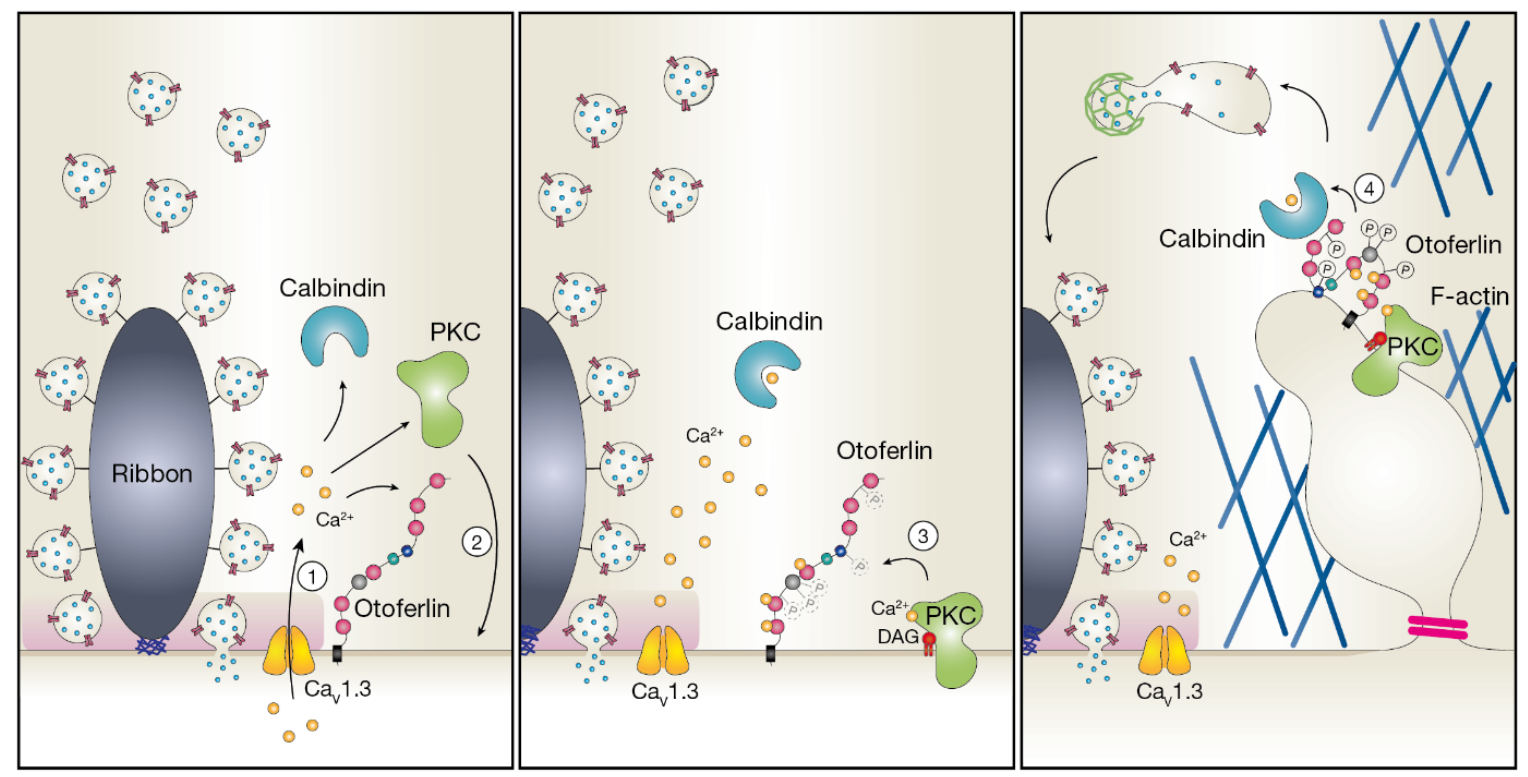

This study provides a molecular mechanism regulating endocytosis at auditory inner hair cell (IHC) ribbon synapses: protein kinase $\mathrm{C} \alpha(\mathrm{PKC} \alpha)$ phosphorylates otoferlin and both localize to endocytic compartments upon PKC $\alpha$ activation; otoferlin interacts with calbindin-D28k and myosin VI in a PKC $\alpha$-dependent manner, forming a $\mathrm{Ca}^{2+}$-dependent signaling complex.

- Strong IHC depolarization leads to activation and targeting of PKC $\alpha$ to endocytic compartments where it colocalizes with otoferlin.

- PKC $\alpha$ interacts with otoferlin in an activity-dependent manner (i.e. upon inner hair cell depolarization) and phosphorylates it at five serine residues.

- Otoferlin interacts with calbindin-D28k in an activity-dependent and PKC-dependent manner.

- PKC $\alpha$ and calbindin-D28k seem not to interact directly.

- PKC $\alpha$ promotes the interaction of otoferlin with calbindin-D28k, which might trigger ultrafast endocytosis in IHCs.

- The interaction of otoferlin with myosin VI is strongly enhanced by PKC $\alpha$ activation. 


\subsubsection{Abstract}

Otoferlin is essential for the fast and indefatigable release of synaptic vesicles at auditory inner hair cell (IHC) ribbon synapses, being involved in exocytic, endocytic and regenerative steps of the synaptic vesicle cycle. Serving diverse functions at this highly dynamic synapse implies that this multi- $\mathrm{C}_{2}$ domain protein is precisely regulated. Here we found protein kinase $\mathrm{C} \alpha$ (PKC $\alpha)$ and otoferlin to colocalize in endocytic recycling compartments upon IHC depolarization and to interact in an activity-dependent manner. In vitro assays confirmed that PKC $\alpha$ can phosphorylate otoferlin at five serine residues, which correlates with increased serine phosphorylation in $<40 \mathrm{~nm}$ proximity to otoferlin in murine IHCs that can be fully blocked by combining PKC and CaMKII inhibitors. Moreover, otoferlin interacts with calbindinD28k in stimulated IHCs, which was precluded when PKC $\alpha$ was inhibited. Similarly, the activity-dependent increase in otoferlin-myosin VI interaction depends on PKC $\alpha$ activation. We propose that upon strong hair cell depolarization, $\mathrm{PKC} \alpha$ phosphorylates otoferlin, thereby enabling it to interact with calbindin-D28k and myosin VI, building a $\mathrm{Ca}^{2+}$-dependent signaling complex that possibly regulates different modes of endocytosis.

Keywords: calbindin / endocytosis / otoferlin / PKC / ribbon synapse

\subsubsection{Introduction}

In the mammalian auditory system, sound encoding between the sensory inner hair cells (IHCs) and the primary auditory neurons occurs with remarkable precision, reliability, and dynamics over prolonged periods of stimulation (Moser \& Beutner, 2000; Glowatzki \& Fuchs, 2002). IHC ribbon synapses are highly specialized for this challenging task, constantly sustaining the pool of fusion-competent vesicles. At physiological temperature, each synapse of a depolarized IHC can sustain a synaptic vesicle (SV) fusion rate of up to 2300 vesicles per second for at least several hundred milliseconds (Strenzke et al, 2016). This high release rate requires the efficient and coordinated retrieval of excess plasma membrane, for which both clathrin-independent and clathrin-dependent modes of endocytosis have been proposed (Neef et al, 2014; Kroll et al, 2019). Crucial proteins for $\mathrm{Ca}^{2+}$-triggered exocytosis in conventional synapses, like SNAREs, synaptotagmins, Munc13 or complexins, are either absent or are dispensable for exocytosis in IHCs (Safieddine \& Wenthold, 1999; Strenzke et al, 2009; Beurg et al, 2010; Nouvian et al, 2011; Reisinger et al, 2011; Vogl et al, 2015). The multi- $C_{2}$ domain protein otoferlin seems to replace some of these proteins and is currently hypothesized to act as the $\mathrm{Ca}^{2+}$ sensor for exocytosis in mature IHCs (Roux et al, 2006; Michalski et al, 2017). Different $O T O F$ mutations lead to almost entirely abolished IHC exocytosis and thus to profound deafness in humans and animal models (Yasunaga et al, 1999, 2000; Roux et al, 
2006; Longo-Guess et al, 2007; Marlin et al, 2010; Pangršič et al, 2010; Reisinger et al, 2011). Otoferlin is involved in vesicle priming and fusion, vesicle replenishment, vesicle reformation from bulk endosomes, active zone clearance, and clathrin-mediated endocytosis (Pangršič et al, 2010; Duncker et al, 2013; Jung et al, 2015a; Strenzke et al, 2016). It has been reported to interact with several proteins involved in the SV cycle, e.g. Rab8b, myosin VI, Cav1.3 calcium channels, the adaptor protein 2 (AP-2) and endophilin A (Roux et al, 2006; Heidrych et al, 2008, 2009; Ramakrishnan et al, 2009; Roux et al, 2009; Johnson \& Chapman, 2010; Zak et al, 2012; Duncker et al, 2013; Ramakrishnan et al, 2014; Vincent et al, 2014; Jung et al, 2015a; Hams et al, 2017; Meese et al, 2017; Kroll et al, 2019). Otoferlin bears six to seven $\mathrm{C}_{2}$ domains, of which at least three likely bind $\mathrm{Ca}^{2+}$ (Meese et al, 2017). Binding of $\mathrm{Ca}^{2+}$ to $\mathrm{C}_{2}$ domains is known to promote or hinder protein interactions, thus it is conceivable that this modular protein might integrate a series of regulatory interactions to finely balance the requirements of exo- and endocytosis at this synapse.

In conventional neuronal synapses, second messenger-activated protein kinases like $\mathrm{Ca}^{2+} /$ calmodulin-dependent protein kinase II (CaMKII), cAMP-dependent protein kinase A (PKA), and protein kinase $\mathrm{C}$ (PKC) tightly and finely regulate synaptic transmission, and their activation correlates with increased transmitter release (Capogna et al, 1995; Hilfiker \& Augustine, 1999). They control the function of the release machinery and the final steps of SV docking/fusion by regulating not only the availability of free SNARE proteins to form the functional fusion machinery but also protein-protein interactions within the release apparatus (reviewed in Turner et al, 1999b; Leenders \& Sheng, 2005). They have also been implicated in presynaptic plasticity via regulation of the refilling of the readily releasable pool of SVs thereby governing the number of release sites and the release probability (Pang et al, 2010; Stevens \& Sullivan, 1998; Leenders \& Sheng, 2005). At IHC synapses, CaMKII $\delta$ was shown to phosphorylate otoferlin, rendering its $\mathrm{C}_{2} \mathrm{~F}$ domain $\mathrm{Ca}^{2+}$-insensitive under physiological conditions (Meese et al, 2017). The regulation of otoferlin's activity by CaMKII $\delta$ may provide a molecular mechanism that influences the kinetics of exocytosis, endocytosis and vesicle replenishment in IHCs.

In this study, we assessed the effects of PKC in IHC synaptic function. Conventional PKCs (cPKCs; $\alpha, \beta$ and $\gamma$ ), the most abundant, structurally comprise a phospholipid-binding diacylglycerol $(\mathrm{DAG}) /$ phorbol ester-binding $\mathrm{C}_{1}$ domain, followed by a $\mathrm{Ca}^{2+}$-binding $\mathrm{C}_{2}$ domain and a C-terminal kinase moiety (reviewed in Rosse et al, 2010; Callender \& Newton, 2017). They require $\mathrm{Ca}^{2+}$ and either membrane-bound DAG or phosphatidylserine (PS) for activation, but can also be activated by other DAG mimetics, resulting in enhanced glutamate release (Castagna et al, 1982; Nishizuka, 1984; Malenka et al, 1986; Shapira et al, 1987; Parfitt \& Madison, 1993; Hori et al, 1999; Yawo, 1999; Brager et al, 2003; Korogod et al, 2007). The DAG/PKC pathway is one of the most potent pathways at presynaptic nerve terminals with its activation resulting in $50-100 \%$ potentiation of spontaneous and action potential- 
induced release (Malenka et al, 1986; Shapira et al, 1987; Lou et al, 2005). cPKC is autoinhibited by a pseudosubstrate sequence in its regulatory domain that sterically blocks the catalytic domain, rendering the kinase inactive and targeting it to the cytosol (Parker \& Murray-Rust, 2004; Newton, 2010; Gould et al, 2011; Antal et al, 2014, 2015). cPKCs are activated in a sequential fashion. Firstly, $\mathrm{Ca}^{2+}$ binding to the $\mathrm{C}_{2}$ domain leads to an increased affinity of cPKC to phospholipids, resulting in its recruitment to membranes, where it then binds to its allosteric activator DAG via the $\mathrm{C}_{1}$ domain. This renders the $\mathrm{CPKC}$ in an open and active form ready to phosphorylate target substrates (Kraft et al, 1982; Sakai et al, 1997; Verdaguer et al, 1999; Sánchez-Bautista et al, 2006; Evans et al, 2006). Besides phosphorylating presynaptic proteins to facilitate exocytosis (Shimazaki et al, 1996; Kataoka et al, 2000; Genoud et al, 2001; Nagy et al, 2002; Barclay et al, 2003; Wierda et al, 2007; Genç et al, 2014; Cijsouw et al, 2014; Jong et al, 2016), PKC is emerging as a central player in vesicular transport pathways, being involved in regulation of membrane trafficking and endocytosis (reviewed in Alvi et al, 2007). For instance, it was shown to regulate the targeting of synaptotagmin IX to endocytic compartments (Haberman et al, 2005).

In this study, we investigated the effects of PKC $\alpha$ activation on the function of the IHC synaptic protein otoferlin, unravelling a $\mathrm{Ca}^{2+}$-controlled signaling complex potentially acting in regulation of endocytosis.

\subsubsection{Results}

\section{PKC $\alpha$ is expressed in the organ of Corti and redistributes to the synaptic region of IHCs upon stimulation where it colocalizes with otoferlin}

PKC is known to regulate presynaptic plasticity, exocytosis and endocytosis in neurons (Shapira et al, 1987; Alvi et al, 2007; Jong et al, 2016). To examine whether PKC is involved in modulating presynaptic function in IHCs, we mapped its subcellular localization and tested if it colocalizes with otoferlin (Figure 3.1 and Figure EV1). We performed immunohistochemistry on acutely dissected organs of Corti from wild-type C57BL/6J (henceforth, WT) mice after the onset of hearing (at P15). Dissections were done in phosphate buffered saline (PBS) solution (no supplemented $\mathrm{Ca}^{2+}$ ). The $\alpha$ isoform was chosen because it is the $\mathrm{CPKC}$ most ubiquitously expressed in other systems and there is only one variant (Kofler et al, 2002). We found PKC $\alpha$ to be expressed both in IHCs and OHCs (Figure EV1A). In IHCs, PKC $\alpha$ was expressed throughout the cell (Figure EV1B), predominantly in the cytosol and to a lesser extent at the plasma membrane, where it partially colocalizes with otoferlin (Figure EV1C-D). 
A

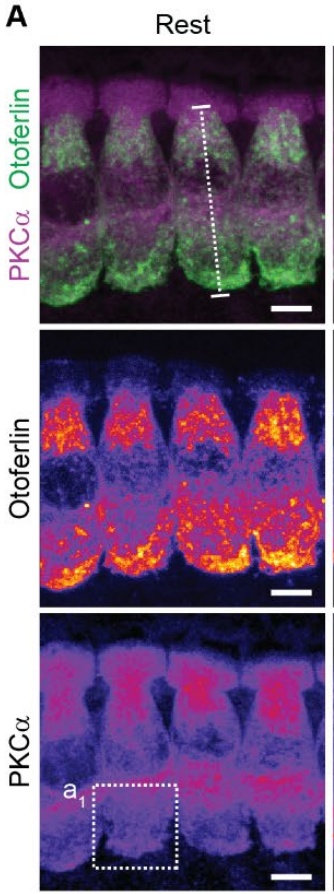

C
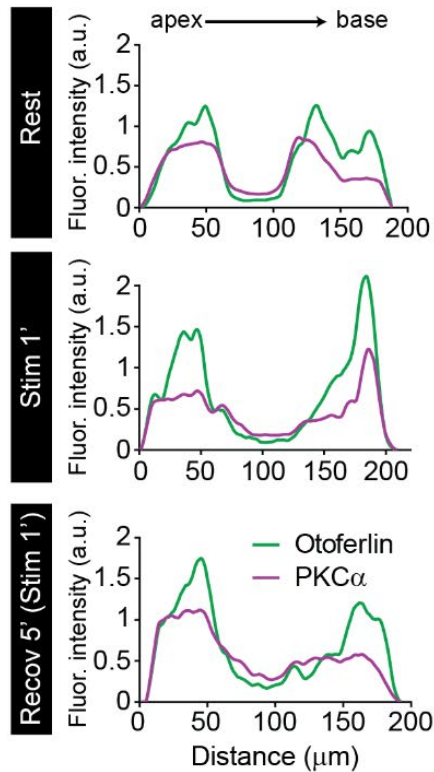

Stim 1'
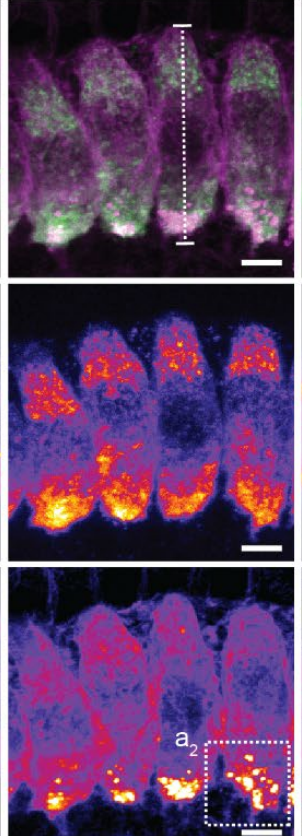

$-8$

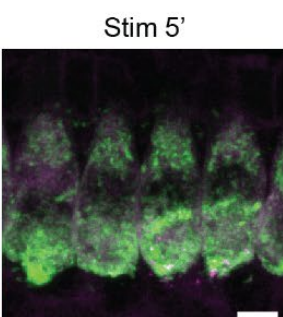

Recov 5' (Stim 1')
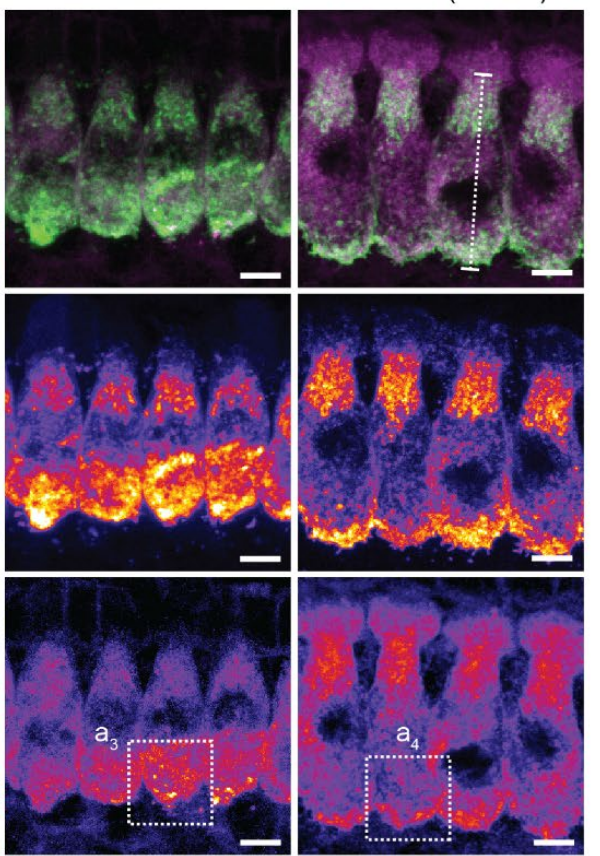

$\min \underset{\max }{2}$
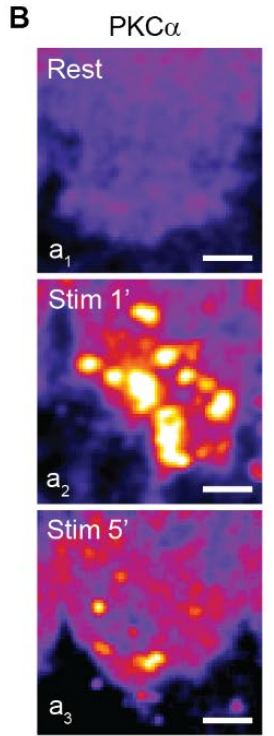

Recov 5' (Stim 1')

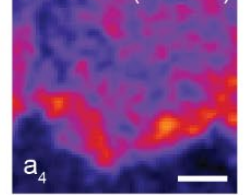

D

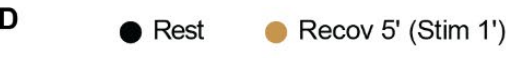

$\min$
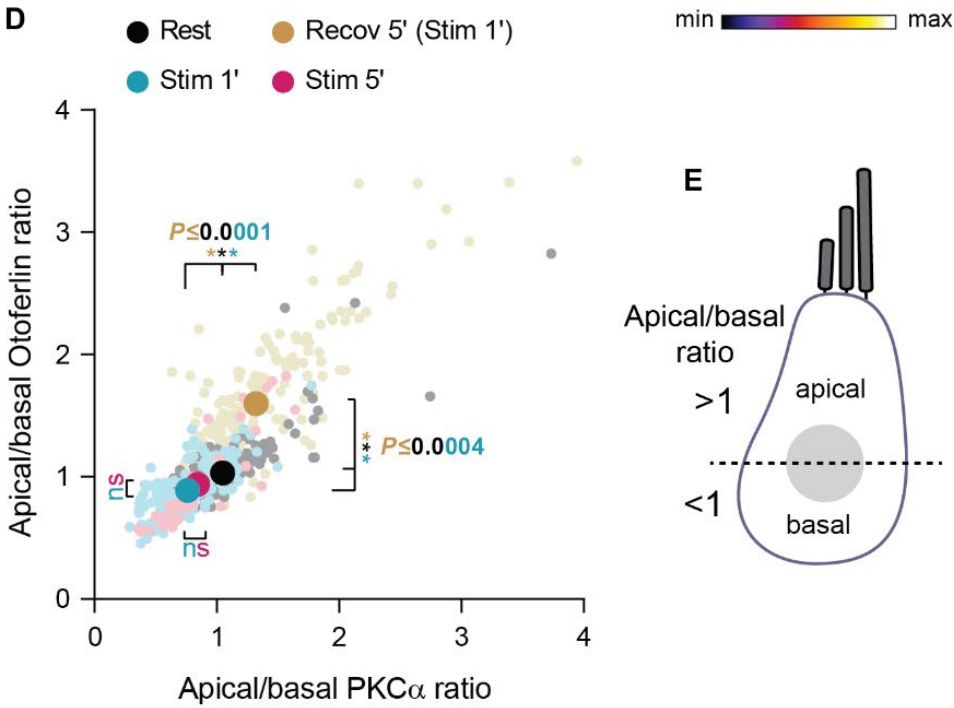

Figure 3.1. PKC $\alpha$ redistributes to base of IHCs upon strong stimulation and PKC $\alpha$ distribution correlates with otoferlin localization.

A High magnification views of representative WT P14-16 IHCs immunolabeled for PKC $\alpha$ and otoferlin, at rest (Rest), after strong stimulation for 1 (Stim 1') and 5 minutes (Stim 5') and after a 5-minute recovery period post 1-minute stimulation (Recov 5' (Stim 1')). For clarity, individual otoferlin and PKC $\alpha$ channels are depicted separately with an intensity-coded lookup table with warmer colors representing higher pixel intensities (middle and bottom panels).

B PKC $\alpha$ immunolabelling (intensity-coded lookup table) in higher magnification views of basal regions of the IHCs labelled in (A) as $a_{1}, a_{2}, a_{3}, a_{4}$. Note that PKC $\alpha$ clustering is maximal at 1 -minute stimulation.

C Fluorescence intensity line profile through the longitudinal axis at the mid-region of representative IHCs labelled in (A), from apex to base (five optical sections). Note that PKC $\alpha$ relocates to the base of the IHCs at Stim 1' where the intensity line profile overlaps with that of otoferlin.

D Correlation of otoferlin and PKC $\alpha$ ratio of apical/basal immunofluorescence (above/below nuclear midline depicted in (E)) indicates a strong localization correlation between PKC $\alpha$ and otoferlin. Mean 
values are displayed with darker colors and bigger symbols. Individual cells are depicted with lighter colors and smaller symbols. Mean averages, sample size and statistical analysis are detailed in Appendix Table S1.

E Schematic representation of an IHC, indicating how the apical/basal ratio of immunofluorescence was determined. Higher immunofluorescence above or below the nuclear midline (dashed line) results in a ratio $>1$ or $<1$, respectively. A ratio $>1$ indicates a shift towards a more apical localization while a ratio $<1$ corresponds to a more basal localization of the protein.

Data information: In (A-B), maximum intensity projections of confocal optical sections. Scale bars: $5 \mu \mathrm{m}(\mathrm{A}), 2$ $\mu \mathrm{m}$ (B). Fluor., fluorescence.

A
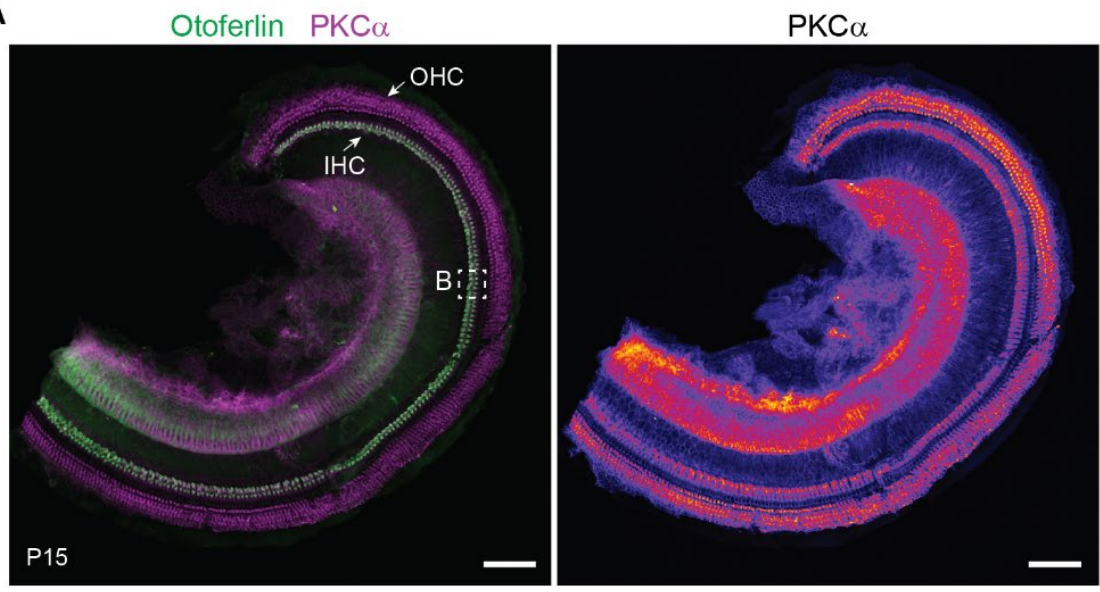

B

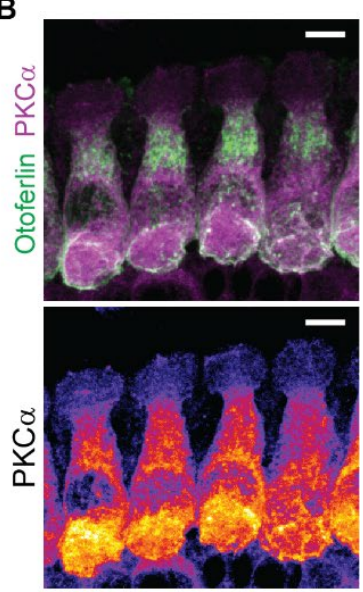

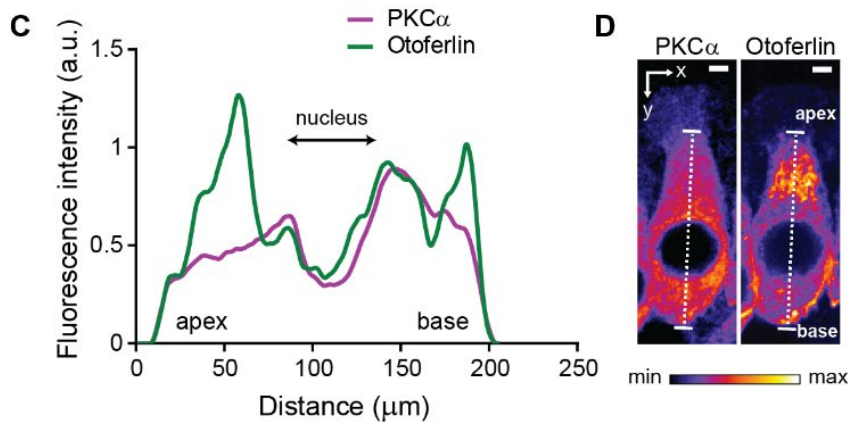

Figure EV1. PKC $\alpha$ is expressed in the organ of Corti, in IHCs and OHCs.

A, B WT organ of Corti (P15) immunolabeled for otoferlin and PKC $\alpha$. (A) Low magnification views. (B) High magnification views of IHCs from the organ of Corti shown in (A). PKC $\alpha$ channel is depicted separately with an intensity-coded lookup table with warmer colors representing higher pixel intensities. IHCs: inner hair cells, OHCs: outer hair cells.

C, D (C) Fluorescence intensity line profile through the longitudinal axis at the mid-region of a representative IHC, from apex to base (five optical sections). (D) Representative IHC used for the fluorescence intensity line profile. PKC $\alpha$ and otoferlin channels depicted separately (intensity-coded lookup table).

Data information: In (A-B), maximum intensity projections of confocal optical sections. (D) Single optical section. Scale bars: $100 \mu \mathrm{m}$ (A), $5 \mu \mathrm{m}(\mathrm{B}), 2 \mu \mathrm{m}$ (D).

Upon $\mathrm{Ca}^{2+}$ influx, PKC typically relocates to the plasma membrane upon $\mathrm{Ca}^{2+}$ influx (Codazzi et al, 2001; Zhao et al, 2006). Using a previously described rest/stimulation/recovery paradigm (Kamin et al, 2014; Revelo et al, 2014), we followed PKC $\alpha$ immunofluorescence in IHCs (Figure 3.1). In resting conditions (1 $\mathrm{min}, 5.36 \mathrm{mM} \mathrm{KCl}$, no supplemented $\mathrm{Ca}^{2+}$ ), 
PKC $\alpha$ immunoreactivity was located almost exclusively in the cytosol. Strong stimulation (1 min, $65.36 \mathrm{mM} \mathrm{KCl}, 2 \mathrm{mM} \mathrm{CaCl}_{2}$ ) resulted in a distinct relocation of PKC $\alpha$ within the base of the IHCs where it accumulated in clusters close to the plasma membrane (Figure 3.1A-B and Figure 3.2). Most PKC $\alpha$ clusters were found near the synaptic ribbons (Figure 3.2A-F) in structures resembling plasma membrane patches and endosomes where it partially colocalized with otoferlin (Figure 3.2E-H). This effect seems to be rather transitory as only few PKC $\alpha$ clusters remained after 5-minute stimulation, and hardly any PKC $\alpha$ clusters persisted after 5minute recovery $\left(5 \mathrm{~min}, 5.36 \mathrm{mM} \mathrm{KCl}, 2 \mathrm{mM} \mathrm{CaCl}_{2}\right.$, following a 1-minute stimulation; Figure 3.1A-B). The relocation of $\mathrm{PKC} \alpha$ to regions close to active zones in a cluster-like appearance seems to occur only for strong stimulations, as no clustering could be observed at milder stimulations inducing less $\mathrm{Ca}^{2+}$ influx $(1 \mathrm{~min}, 25 \mathrm{mM} \mathrm{KCl}, 2 \mathrm{mM} \mathrm{CaCl} 2$; Figure EV2A). Interestingly, the trafficking of otoferlin seems to follow that of PKC $\alpha$ (Figure 3.1A, $\mathrm{C}-\mathrm{E})$, pointing towards a probable interaction of the two proteins. At rest, otoferlin immunofluorescence was found throughout the cell in the cytoplasm and at the plasma membrane whereas PKC $\alpha$ seems to be expressed mainly in the cytoplasm with rather weak plasma membrane localization (Figure 3.1C, upper panel; Figure 3.1D, apical/basal PKC $\alpha$ ratio: $1.05 \pm 0.03$; mean \pm standard error of the mean, s.e.m.). After 1-minute stimulation, both PKC $\alpha$ and otoferlin localized more towards the basal region of the IHCs when compared to resting conditions (Figure 3.1C, middle panel; Figure 3.1D, apical/basal ratio $<1$ ), while both were found more apically after letting the cells recover for 5 minutes (Figure 3.1C, bottom panel; Figure 3.1D, apical/basal ratio $>1$ ).

In order to find out if the observed clustering of PKC $\alpha$ close to the plasma membrane of IHCs is coherent with its activation, we assessed its localization after pharmacological PKC $\alpha$ activation. In many cell types treatment with phorbol 12-myristate 13-acetate (PMA), a PKC agonist that mimics DAG and strongly binds cPKCs (Takekoshi et al, 1995), induced the recruitment of PKC to membranes (Hermelin et al, 1988; Huang et al, 1997; Feng et al, 1998, 2000; Tardif et al, 2002; González et al, 2003; Schechtman et al, 2004; Wu et al, 2006; Cordey $\&$ Pike, 2006). When we treated organs of Corti with PMA, PKC $\alpha$ redistributed to the plasma membrane throughout the cell (Figure EV2B-C) with the stronger effect observed at 15minute incubation (Figure EV2B-C, PMA 15'). Moreover, PMA treatment for 5 minutes resulted in enrichment of otoferlin immunofluorescence in large patches at the base of the hair cells (Figure EV2B, PMA 5') and enhanced colocalization of PKC $\alpha$ and otoferlin at the basolateral plasma membrane could be observed for 15-minute incubation (Figure EV2B, PMA 15').

Altogether, these results indicate co-trafficking of PKC $\alpha$ and otoferlin upon PKC $\alpha$ activation (either pharmacologically or following strong stimulation) and thus point towards a possible activity-dependent interaction of the two proteins. 

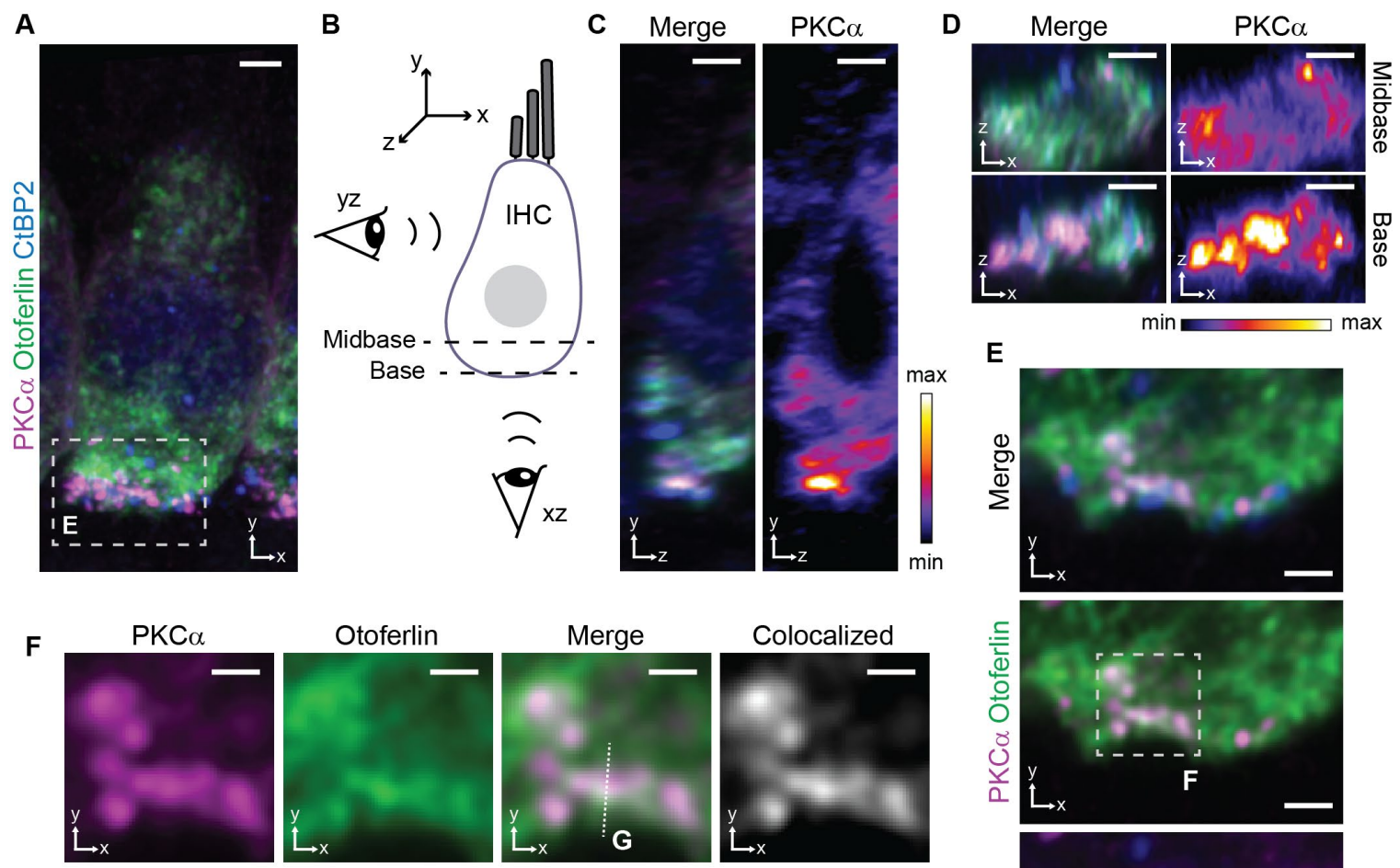

G
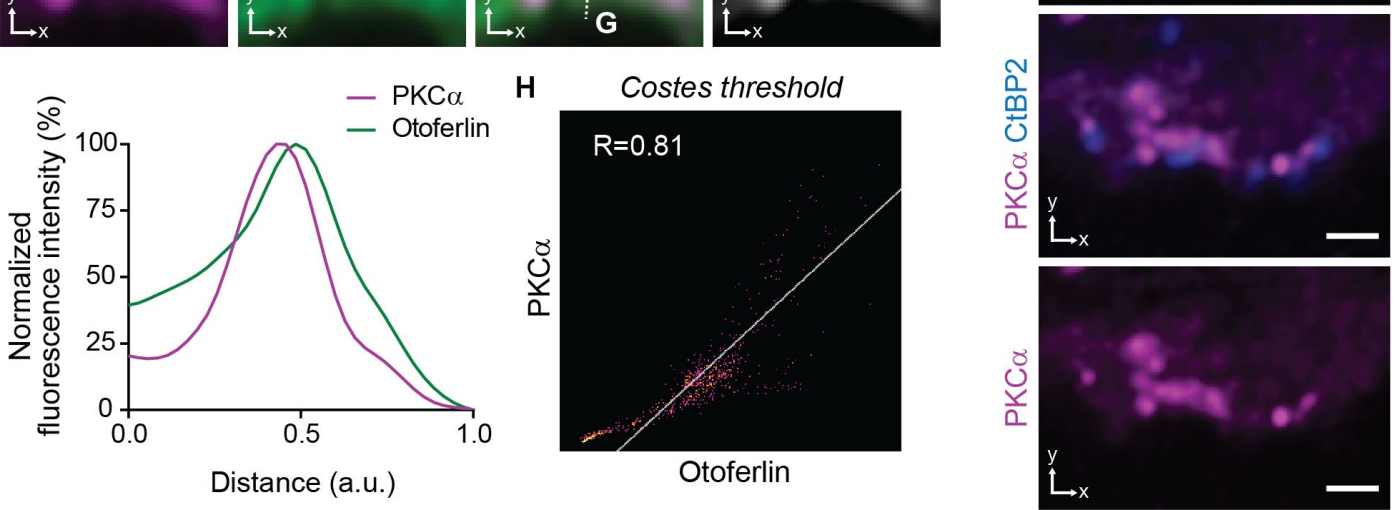

Figure 3.2. PKC $\alpha$ redistributes to the base of IHCs after strong stimulation, where it is found near the synaptic ribbons and partially colocalizes with otoferlin.

A High magnification view of a representative WT P15 IHC immunolabeled for PKCa (magenta), otoferlin (green) and the ribbon marker CtBP2 (blue), after strong stimulation for 1 minute.

B-D Orthogonal views of the IHC displayed in (A). (B) Schematic representation of an IHC, with illustration of side (yz; C) and bottom (xz; D) views, for clarity. In (C-D), individual PKC $\alpha$ channel is depicted separately (right panels) with an intensity-coded lookup table.

E Higher magnification view of the basal region of the IHC displayed in (A), showing PKC $\alpha$ accumulations in regions close to the ribbons (immunolabelled with an antibody against CtBP2, blue) and in endosome-like structures.

F-H Colocalization analysis for otoferlin (green) and PKC $\alpha$ (magenta) channels for the area labelled in E. (F) Single image plane with individual (first two panels) and merged (third panel) channels. Pixels with positive signals for both channels are shown in white (forth panel). (G) PKC $\alpha$ and otoferlin intensity profiles through the dashed line in F. (H) Scatterplot of PKC $\alpha$ and otoferlin pixel intensities and calculated Pierson's correlation coefficient using the Costes automatic thresholding method.

Data information: In (A), maximum intensity projection of confocal optical sections. In (C-F), single confocal optical sections. Scale bars: $2 \mu \mathrm{m}$ (A, C-D), $1 \mu \mathrm{m}$ (E), $0.5 \mu \mathrm{m}$ (F). IHC, inner hair cell. 

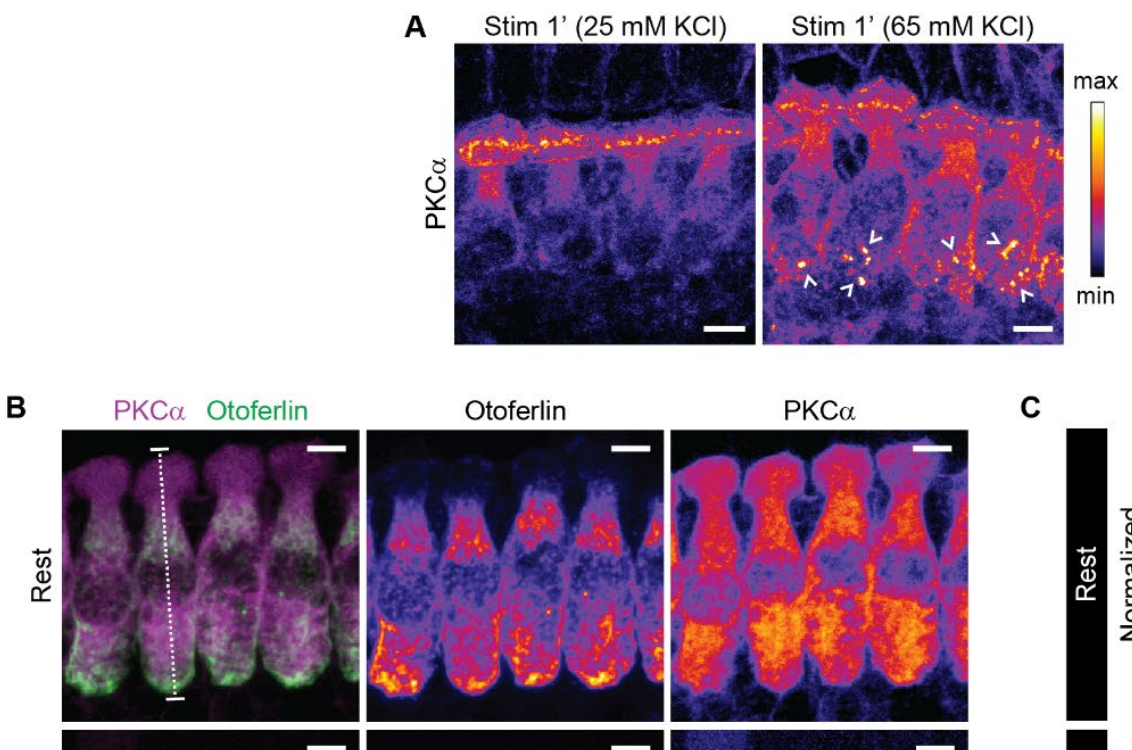

\section{C}
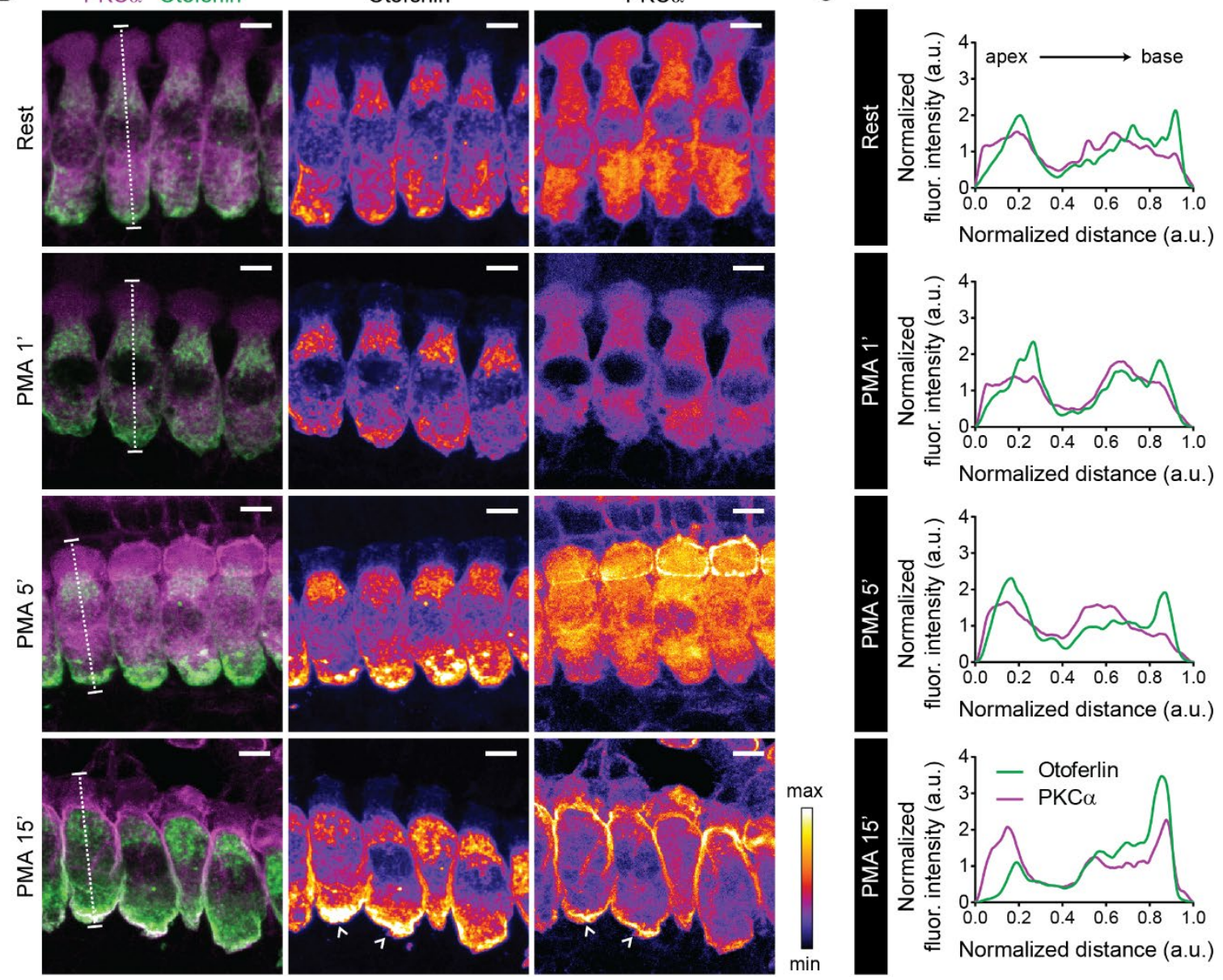

Figure EV2. PKC $\alpha$ redistributes upon strong stimulation and treatment with PMA.

A High magnification views of representative WT P15-16 IHCs immunolabeled for PKC $\alpha$, after mild (25 $\mathrm{mM} \mathrm{KCl})$ and strong $(65 \mathrm{mM} \mathrm{KCl})$ stimulations for 1 minute. PKC $\alpha$ staining is depicted with an intensity-coded lookup table with warmer colors representing higher pixel intensities.

B High magnification views of representative WT P15-16 IHCs immunolabeled for PKC $\alpha$ and otoferlin, at rest (Rest), and after treatment with the PKC activator PMA for 1 (PMA 1'), 5 (PMA 5') and 15 (PMA 15') minutes. Individual otoferlin and PKC $\alpha$ channels are depicted separately with an intensitycoded lookup table with warmer colors representing higher pixel intensities.

C Fluorescence intensity line profile through the longitudinal axis at the mid-region of representative IHCs labelled in (B), from apex to base (five optical sections).

Data information: In (A-B), maximum intensity projections of confocal optical sections. Scale bars: $5 \mu \mathrm{m}$. 


\section{PKC $\alpha$ interacts with otoferlin in IHCs}

We next investigated a potential interaction of PKC $\alpha$ and otoferlin in IHCs. We first performed a proximity ligation assay (PLA), which allows in situ detection of endogenous protein interactions with single molecule resolution, detecting a $<40 \mathrm{~nm}$ distance of antibodylabeled proteins (Figure 3.3A-B). This assay was previously established for rat IHCs with the reported interaction pair otoferlin-myosin VI and was validated here in mouse IHCs (Appendix Figure S1). The PLA for otoferlin and PKC $\alpha$ performed in explanted organs of Corti of P14-16 mice in resting conditions resulted in few fluorescent puncta distributed throughout the IHC (Figure 3.3A-B). When the same PLA was performed after 1-minute stimulation, we saw a $>4$-fold increase in PLA fluorescence intensity $(442 \pm 28 \%, n=141$ IHCs) when compared to resting conditions $(100 \pm 7 \%, \mathrm{n}=122$ IHCs), pointing to an interaction of the proteins upon strong IHC stimulation. The intensity of the PLA puncta dropped to $178 \pm 7 \%$ ( $\mathrm{n}=112$ IHCs) during a 5-minute recovery period, indicating a rather short-living otoferlin-PKC $\alpha$ complex (Figure 3.3A-B).

Given that a positive PLA signal could potentially result from an indirect interaction via scaffolding proteins, we assessed whether otoferlin and $\mathrm{PKC} \alpha$ interact directly in vitro (Figure 3.3C-D). In a first approach, we co-transfected HEK293T cells with HA-tagged full-length otoferlin (mCherry-P2A-mOtof-HA) and GFP-tagged PKC $\alpha$ (eGFP-PKC $\alpha$; Figure 3.3C, upper panel) and performed anti-HA and anti-GFP co-immunoprecipitation (Co-IP) assays. Western blotting of immunoprecipitated samples of HA IPs, where otoferlin-HA was used as bait, revealed a band of $-105 \mathrm{kDa}$ in the eluate corresponding to GFP-PKC $\alpha$ when immunoblotted against GFP (Figure 3.3C, bottom left panel). Conversely, a band of -240 $\mathrm{kDa}$ corresponding to otoferlin-HA was detected with an anti-HA antibody, when GFPPKC $\alpha$ was used as bait in GFP IPs. The faint bands in both situations suggest a weak interaction of the two proteins, possibly because the cells were harvested in conditions with weak PKC activation. In a second approach, we ran pull-down assays from organ of Corti homogenates which we loaded onto HA beads enriched with otoferlin-HA protein previously expressed in HEK293T cells (Figure 3.3D, upper panel). When we immunoblotted for PKC $\alpha$, a strong band of $-77 \mathrm{kDa}$ was evident in the eluate; the same band was absent in control experiments with HEK-expressed HA peptide (Figure 3.3D, bottom panel). Both in vitro assays indicate that otoferlin and PKC $\alpha$ can interact directly. 

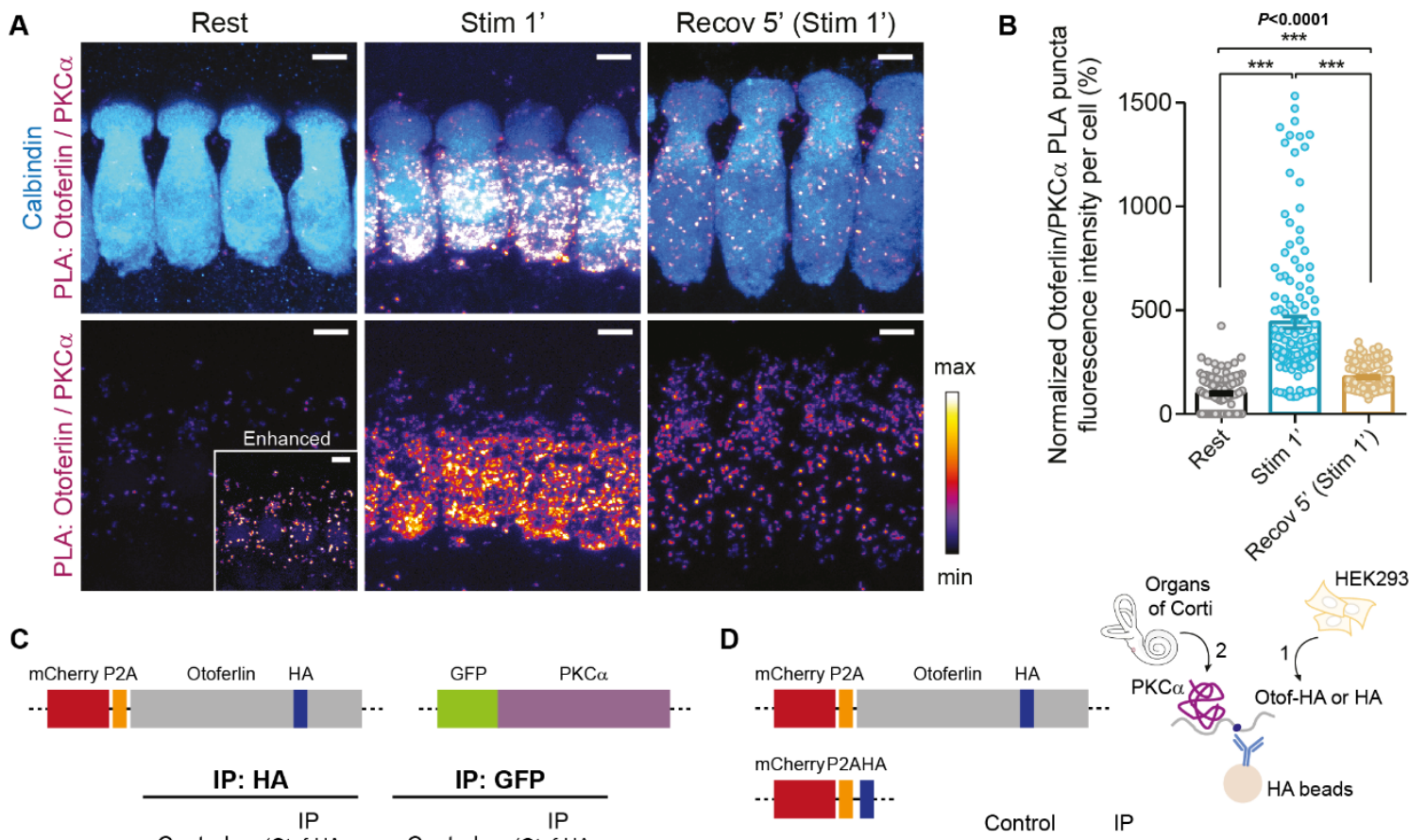

C
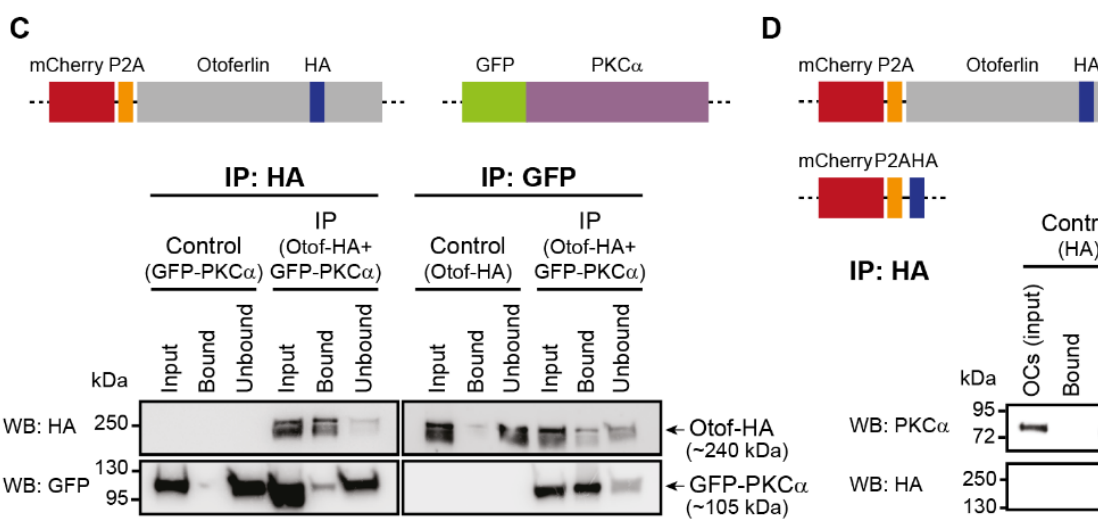

IP: HA
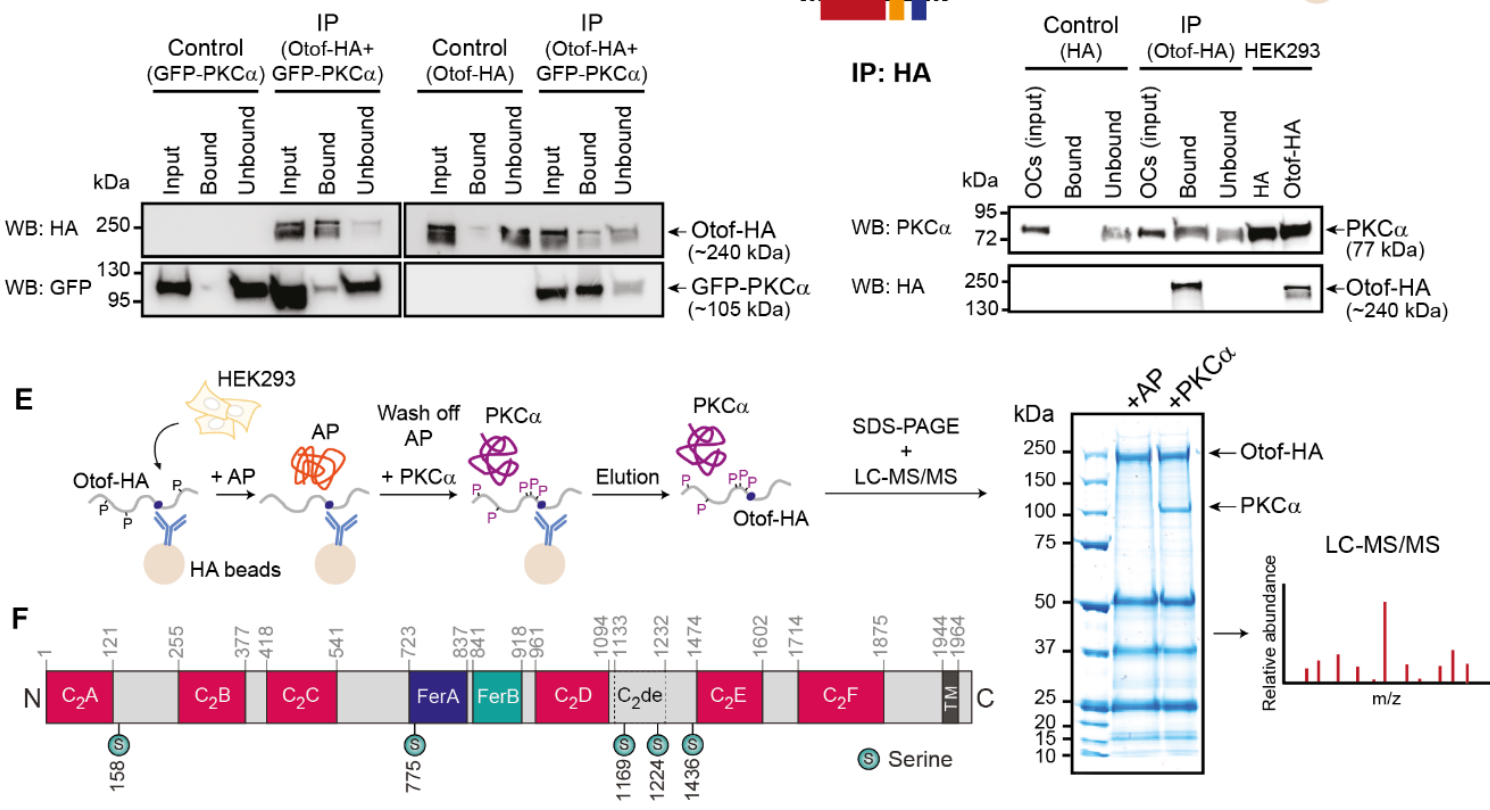

Figure 3.3. PKC $\alpha$ and otoferlin interact in IHCs.

A, B PLA for otoferlin and PKC $\alpha$ performed on WT P15 IHCs at rest, after strong stimulation for 1 (Stim $1^{\prime}$ ) and 5 minutes (Stim 5'), and after a 5-minute recovery period post stimulation (Recov 5' (Stim 1')). (A) High magnification views of representative PLAs: calbindin (blue) was used as IHC marker; PLA channel is depicted with an intensity-coded lookup table with warmer colors representing higher pixel intensities. (B) Average otoferlin/PKC $\alpha$ PLA puncta fluorescence intensity per cell for all conditions, normalized to the resting condition. Individual cells are depicted with lighter colors and open symbols. See Appendix Figure S2A and C for control PLAs.

C Representative immunoblot showing results of anti-HA and anti-GFP co-immunoprecipitation from lysates of HEK293T cells co-transfected with otoferlin-HA and GFP-PKC $\alpha$. Samples were probed for HA and GFP. Upper panel depicts constructs used in binding assays.

D Representative immunoblot showing results from pull-down assay from organs of Corti loaded onto anti-HA beads with previously bound otoferlin-HA expressed in HEK293T cells. Samples were probed for $\mathrm{HA}$ and PKC $\alpha$. Upper left panel depicts constructs used in binding assays. Upper right panel depicts scheme of the assay. 
E Schematic representation of the in vitro phosphorylation assay and subsequent mass spectrometry analysis to assess PKC $\alpha$-induced phosphosites on otoferlin. Alkaline phosphatase (AP) was used to remove any residual phosphate groups and obtain dephosphorylated otoferlin. After incubation with AP + PKC $\alpha$ or with AP only, samples were loaded onto a SDS-PAGE gel, otoferlin bands were excised, digested and analyzed by LC-MS/MS. For annotated MS/MS spectra of detected phosphosites, LCMS/MS profiling of phosphopeptides and mapping of sites in the otoferlin sequence refer to Appendix Figures S3-S9.

F Position of the phosphorylation sites in the otoferlin sequence (mouse, isoform 4, NP_001300696.1) determined by LC-MS/MS. For mapping of sites in the otoferlin sequence refer to Appendix Figure S10.

Data information: In (A), maximum intensity projections of confocal optical sections. Scale bars: $5 \mu \mathrm{m}$. In (B), data are displayed as mean \pm s.e.m.; ${ }^{* * *} \mathrm{P} \leq 0.001$ (Kruskal-Wallis test followed by Dunn's multiple comparison test); mean averages, sample size and statistical analysis are detailed in Appendix Table S1. In (A-B), only puncta inside the cells were considered for quantification purposes; quantification was performed in Imaris as described in Materials and Methods. PLA, proximity ligation assay. IHC, inner hair cell. Otof, otoferlin.

An in vitro assay where immobilized otoferlin-HA was incubated with recombinant PKC $\alpha$ was conducted to test if PKC $\alpha$ can phosphorylate otoferlin (Figure 3.3E). LC-MS/MS analysis (Appendix Figures S3-S10) revealed phosphorylation of otoferlin at five serine residues: S158, S775, S1169, S1224 and S1436 (otoferlin variant 4, NP_001300696.1) (Figure 3.3F). All phosphorylation sites were found to be conserved between mammalian and non-mammalian otoferlin orthologs, with the exception of $\mathrm{S} 1169$ at the $\mathrm{C}_{2}$ de domain conserved only among mammalian species (Appendix Figure S11). Interestingly, none of the phosphorylated serine residues is located in one of the main six $\mathrm{C}_{2}$ domains; yet, two were found in the $\mathrm{C}_{2}$ de domain, a putative $\mathrm{C}_{2}$ domain with poor conservation of sequence and secondary structure elements among species. Phosphorylation at S775 in the FerA domain could possibly alter the interaction of FerA with membranes in the presence of $\mathrm{Ca}^{2+}$ (Harsini et al, 2018). It is noteworthy that three of the five positions (S158, S775, S1224) match phosphorylation sites retrieved by different kinase-specific, sequence- and structure-based prediction tools (Appendix Figure S12 and Appendix Table S2).

In the absence of otoferlin hardly any membrane turnover takes place in IHCs due to the abolishment of fast exocytosis (Roux et al, 2006). Here, we used knock-out mice (Otof ${ }^{-/}$) (Reisinger $e t a l, 2011$ ) to find out if the disaggregation of PKC $\alpha$ immunofluorescence clusters depends on proper endocytic and vesicle processes (Figure 3.4). In Otof ${ }^{-/}$IHCs, although PKC $\alpha$ immunofluorescence levels were only slightly altered when compared to WT IHCs (WT: $100 \pm 2 \%, \mathrm{n}=233$ IHCs vs. Otof ${ }^{-1}$ : $92 \pm 1 \%, \mathrm{n}=205$ IHCs; ${ }^{* *} P=0.0054$, Mann-Whitney two-tailed t-test), the localization of PKC $\alpha$ was shifted towards the base of the cell (apical/basal ratio: WT $1.06 \pm 0.03$ vs. Otof ${ }^{-/} 0.66 \pm 0.02$; Figure $\left.3.4 \mathrm{~A}-\mathrm{C}\right)$. Upon high potassium $\left(\mathrm{K}^{+}\right)$ stimulation, PKC $\alpha$ relocated to the base of $\mathrm{Otof}^{-1}$ IHCs (Figure 3.4D-G) in a similar fashion to what happened in WT IHCs (Figure 3.1A-D). This indicates that exocytosis seems not to be required for the trafficking of PKC $\alpha$ towards the plasma membrane. However, after 5- 
minute stimulation and during recovery (following 1-minute stimulation), PKC $\alpha$ immunofluorescence was still evident at endosomal structures and at the basolateral plasma membrane in $\mathrm{Otof}^{--}$IHCs (Figure 3.4D-E). Given that otoferlin is not only involved in exoendocytosis coupling but is also required for proper vesicle reformation from recycling endosomes, it is probable that these plasma membrane patches and endosomes are not converted to smaller vesicles as quickly as in the presence of otoferlin, reassuring that PKC $\alpha$ indeed persists in these endosomal compartments in $\mathrm{Otof}^{-/}$IHCs.
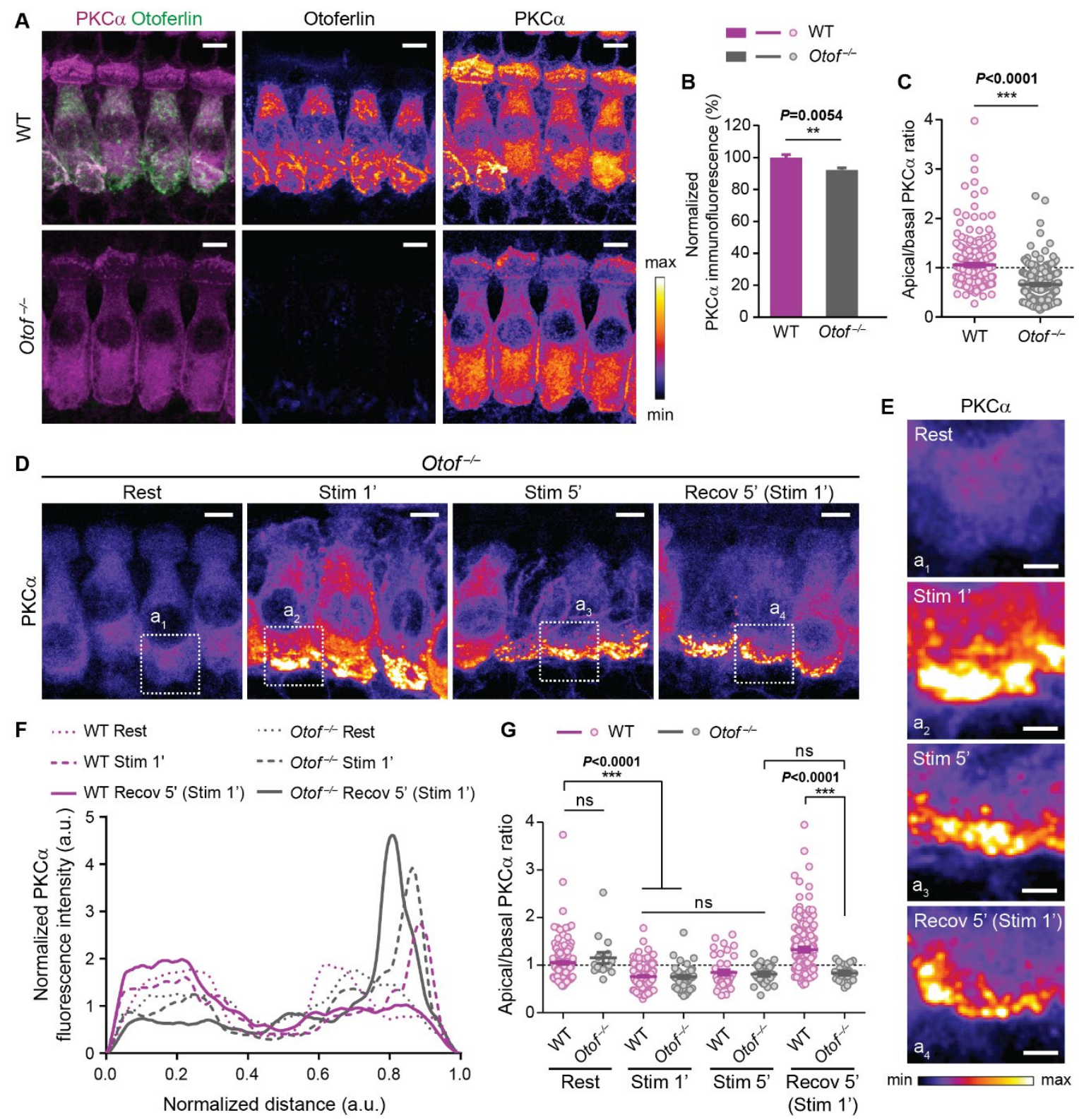

Figure 3.4. PKC $\alpha$ subcellular distribution is affected in otoferlin knock-out IHCs.

A-C PKC $\alpha$ immunofluorescence in Otof ${ }^{-1}$ compared to WT P15-16 IHCs. (A) High magnification views of representative WT B6 and Otof ${ }^{-/}$P14-P16 IHCs immunolabeled for PKC $\alpha$ (magenta) and otoferlin (magenta). Otoferlin and PKC $\alpha$ channels are depicted separately with an intensity-coded lookup table with warmer colors representing higher pixel intensities. (B) Quantification of overall PKC $\alpha$ 
immunofluorescence. (C) Apical/basal PKC $\alpha$ immunofluorescence (above/below nuclear midline). Number of cells in (C) also apply to (B).

D, E PKC $\alpha$ distribution in $\mathrm{Otof}^{--} \mathrm{P} 14-16$ IHCs for all indicated conditions. (D) High magnification views of representative Otof ${ }^{-/}$P15-16 IHCs immunolabeled for PKC $\alpha$. (B) Higher magnification views of basal regions of the Otof $^{-1-}$ IHCs labelled in (D) as $\mathrm{a}_{1}, \mathrm{a}_{2}, \mathrm{a}_{3}$, $\mathrm{a}_{4}$. For clarity, an intensity-coded lookup table was used.

F, G Comparison of PKC $\alpha$ distribution in WT and Otof ${ }^{-1}$ P14-16 IHCs. (F) Fluorescence intensity line profile through the longitudinal axis at the mid-region of representative WT and Otof ${ }^{-1-}$ IHCs, from apex to base (five optical sections). (G) Comparison of apical/basal PKC $\alpha$ immunofluorescence (above/below nuclear midline) among the different experimental conditions.

Data information: In (A, D-E), maximum intensity projections of confocal optical sections. Scale bars: $5 \mu \mathrm{m}$ (A, D), $2 \mu \mathrm{m}$ (E). In (B-C, G), data are displayed as mean \pm s.e.m.; ns $P>0.05$, ${ }^{* *} \mathrm{P} \leq 0.01,{ }^{* *} \mathrm{P} \leq 0.001$; mean averages, sample size and statistical analysis are detailed in Appendix Table S1. In (C, G) individual cells are depicted with lighter colors and open symbols. Rest, resting; Stim 1', 1-minute stimulation; Stim 5', 5-minute stimulation; Recov 5' (Stim 1'), 5-minute recovery after 1-minute stimulation. IHC, inner hair cell.

\section{Activity-dependent phosphorylation of otoferlin or otoferlin interactors by $\mathrm{PKC} \alpha$}

To test whether otoferlin and/or proteins interacting with otoferlin are phosphorylated and to assess if the phosphorylation of otoferlin is activity-dependent in vivo, Meese et al (2017) applied a PLA to find phosphoserine residues in $<40 \mathrm{~nm}$ distance from otoferlin in rat IHCs. Upon stimulation with high $\mathrm{K}^{+}$the PLA signal increased when compared to resting conditions, and this effect could be only partially blocked by the CaMKII inhibitor KN-93 (Meese et al, 2017; Figure 10B-C), suggesting the involvement of other kinases in the regulation of synaptic function through phosphorylation of otoferlin in mammalian IHCs.

We sought to assess whether the increase in phosphorylation of otoferlin or otoferlin-associated proteins is complemented by PKC in mouse IHCs (Figure 3.5). We first stimulated the cells (with $65.36 \mathrm{mM} \mathrm{KCl}, 2 \mathrm{mM} \mathrm{CaCl}$ ) and observed an increase in PLA signal that peaked at 5minute stimulation (Rest: $100 \pm 11 \%, \mathrm{n}=100$ IHCs vs. Stimulation 1': $234 \pm 13 \%, \mathrm{n}=37$ IHCs vs. Stimulation 5': $438 \pm 38 \%, \mathrm{n}=52$ IHCs; ${ }^{* * *} P<0.0001$, Kruskal-Wallis test followed by Dunn's multiple comparison test; Figure 3.5). Pre-incubation with the PKC inhibitor bisindolylmaleimide I (BIM I) for 15 minutes prior to 5-minute stimulation blocked the stimulation-dependent increase in PLA signal to a large extent (BIM I + Stimulation 5': $122 \pm 2 \%, \mathrm{n}=34$ IHCs; ${ }^{* *} P=0.0013$ vs. Stimulation $\left.5^{\prime}\right)$. Moreover, pre-incubation with BIM I and KN-93 completely blocked this effect (BIM I+KN-93 + Stimulation 5': 97 $\pm 4 \%, n=50$ IHCs; ${ }^{* * *} P<0.0001$; Figure 3.5). Treatment with the PKC $\alpha$ activator PMA led to an increase in PLA signal (PMA 5': 157 $\pm 4 \%, \mathrm{n}=66$ IHCs and PMA 15': 139 $\pm 6 \%, \mathrm{n}=48$ IHCs; ${ }^{* * *} P \leq 0.0002$ vs. Rest). This effect could only be seen for longer incubations times (PMA 1': $77 \pm 7 \%, \mathrm{n}=61 \mathrm{IHCs}$; ns $P=0.3464$ vs. Rest; Figure 3.5) likely due to extracellular application and thus the time needed for PMA to flip to the inner leaflet of the lipid membrane. The PMA-induced increase in PLA signal was not as evident as for 1 - and 5-minute high $\mathrm{K}^{+}$ stimulations. Since PMA strongly activates PKC $\alpha$ even in the absence of $\mathrm{Ca}^{2+}$, the finding that phosphorylation by PKC $\alpha$ is enhanced under depolarizing conditions suggests that $\mathrm{Ca}^{2+}$ might 
be able to strengthen the interaction of otoferlin and PKC $\alpha$, likely via binding to their $\mathrm{C}_{2}$ domains. Altogether, these data indicate that the activity-dependent phosphorylation of otoferlin and/or its interactors in mouse IHCs relies on the combined action of PKC $\alpha$ and CaMKIII.

A

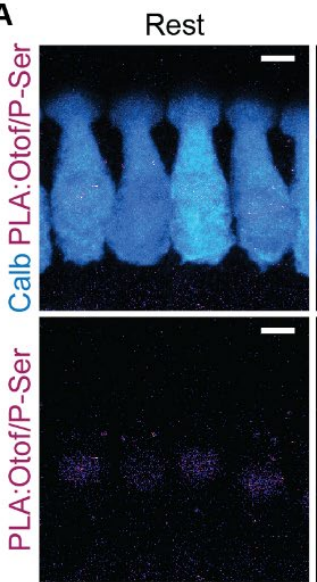

Stim 1'
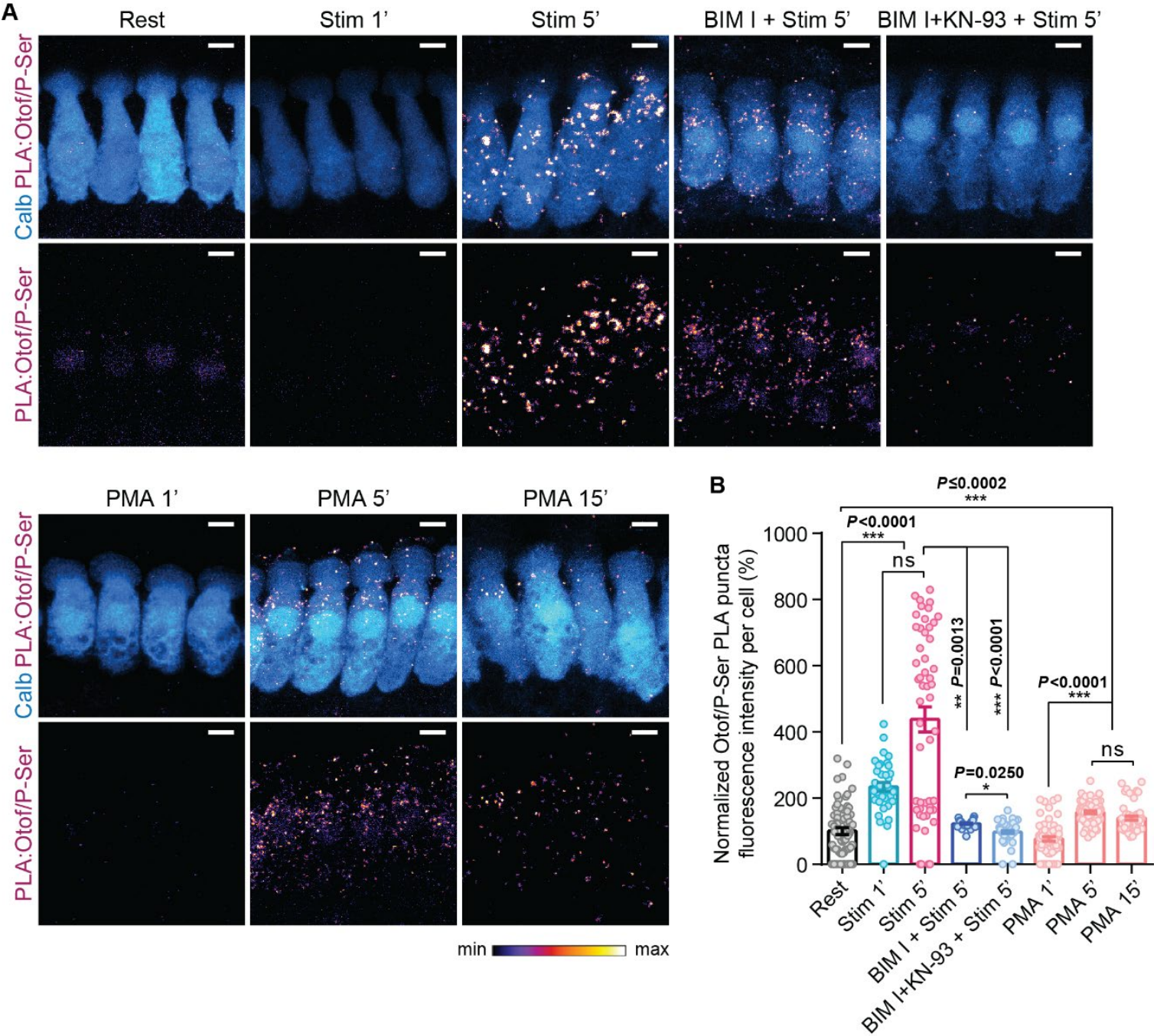

Figure 3.5. Otoferlin phosphorylation is strongly promoted by hair cell stimulation and can be blocked by combined inhibition of PKC and CaMKII.

A, B PLA for otoferlin and phosphoserine residues performed on WT P15-16 IHCs at rest, after stimulation, after incubation with BIM I (PKC inhibitor) or with BIM I and KN-93 (CaMKII inhibitor), and after incubation with PMA (PKC activator). (A) High magnification views of representative PLAs; Calbindin (blue) was used as IHC marker; PLA channel is depicted with an intensity-coded lookup table with warmer colors representing higher pixel intensities. (B) Average otoferlin/phosphoserine PLA puncta fluorescence intensity per cell, normalized to the resting condition. Individual cells are depicted with lighter colors and open symbols. See control PLAs in Appendix Figure S2A and B.

Data information: In (A), maximum intensity projections of confocal optical sections. Scale bars: $5 \mu \mathrm{m}$. In (B), data are displayed as mean \pm s.e.m.; ns $P>0.05,{ }^{*} P \leq 0.05,{ }^{* *} P \leq 0.01,{ }^{* * *} P \leq 0.001$ (Kruskal-Wallis test followed by Dunn's multiple comparison test); mean averages, sample size and statistical analysis are detailed in Appendix Table S1. Rest, resting; Stim 1', 1-minute stimulation; Stim 5', 5-minute stimulation; BIM I + Stim 5', Incubation with BIM I prior to 5-minute stimulation; BIM I+KN-93 + Stim 5', Incubation with BIM I and KN93 prior to 5-minute stimulation; PMA 1', 1-minute incubation with PMA; PMA 5', 5-minute incubation with 
PMA; PMA 15', 15-minute incubation with PMA. IHC, inner hair cell. Otof, otoferlin. P-Ser, phosphoserine. Calb, calbindin. PLA, proximity ligation assay.

\section{Otoferlin interacts with myosin VI in a PKC $\alpha$-dependent manner, but not with Vglut3}

To narrow down the cellular pathways potentially affected by the PKC $\alpha$-mediated regulation of otoferlin, we tested an activity-dependent interaction of otoferlin with candidate proteins.

Firstly, we assessed if the interaction of otoferlin with its reported interaction partner myosin VI (Heidrych et al, 2009; Roux et al, 2009) is influenced by IHC stimulation and PKC activation (Figure 3.6A-C). After stimulation with high $\mathrm{K}^{+}$for 1 minute, not only did myosin VI immunofluorescence levels increase significantly (Rest: 100土 5\%, $\mathrm{n}=43$ IHCs vs. Stimulation 1': $138 \pm 3 \%, \mathrm{n}=44$ IHCs; ${ }^{* *} P<0.0001$; Figure $\left.3.6 \mathrm{~A}-\mathrm{B}\right)$ but the PLA signal for otoferlin and myosin VI increased even more (Rest: 100 $\pm 3 \%, \mathrm{n}=265$ IHCs vs. Stimulation 1': $173 \pm 4 \%, \mathrm{n}=170$ IHCs; $P<0.0001$; Figure 3.6C-D). The PLA signal remained high during a 5 -minute recovery period following a 1-minute stimulation, indicating that the interaction persists in this time frame (Recovery 5' (Stimulation 1'): $183 \pm 4 \%, \mathrm{n}=153$ IHCs, $P>0.9999$ vs. Stimulation 1'). Incubation with BIM I prior to 1-minute high $\mathrm{K}^{+}$stimulation led to a complete abolishment of the stimulation-induced increase in PLA signal (BIM I + Stimulation 1': $102 \pm 6 \%, n=37$ IHCs). Treatment with PMA also resulted in an increase in PLA signal (PMA 5’: 133 $\pm 3 \%, \mathrm{n}=122$ IHCs and PMA 15’: 149 $\pm 9 \%, \mathrm{n}=96$ IHCs; ${ }^{* * *} P<0.0001$ vs. Rest; Kruskal-Wallis test followed by Dunn's multiple comparison test; Figure 3.6C-D). These results support the notion that the interaction of otoferlin and myosin VI is strongly PKCdependent.

We then followed Vglut3, the vesicular glutamate transporter in IHCs, to probe for a potential regulatory role of PKC in exocytosis (Figure 3.6E-I). A 1-minute stimulation with high $\mathrm{K}^{+}$led not only to an increase in Vglut3 immunofluorescence (Rest: $100 \pm 2 \%, n=89$ IHCs vs. Stimulation 1': $163 \pm 8 \%, \mathrm{n}=92$ IHCs; ${ }^{* * *} P<0.0001$ vs. Rest) but also to a relocation of the protein to the basal region of the IHCs (apical/basal ratio: Rest: $1.12 \pm 0.06$ vs. Stimulation 1': $0.83 \pm 0.04$; ${ }^{* * *} P<0.0001$ vs. Rest) with a strong localization to the basolateral plasma membrane (Figure 3.6E, second panel). This likely reflects uncovering of the epitope and the transport of distal SVs to membrane-proximal sites with translocation of Vglut3 from SVs to the active zone membrane during exocytosis. After a 5-minute recovery period the levels (Recovery 5': $118 \pm 2 \%, \mathrm{n}=71 \mathrm{IHCs}$ ) and localization of Vglut3 (apical/basal ratio: Recovery 5': $1.19 \pm 0.08$ ) returned to initial values (Figure 3.6E-G). A PLA for otoferlin and Vglut3 was positive but no change in intensity was registered (Rest: $100 \pm 2 \%, \mathrm{n}=78 \mathrm{IHCs}$ vs. Stimulation 1': $104 \pm 1 \%, \mathrm{n}=146$ IHCs vs. Recovery 5': 95 $\pm 2 \%, \mathrm{n}=93$ IHCs; Figure 3.6H-I). This might be explained by the fact that both proteins are known to localize to common structures in IHCs (Strenzke et al, 2016) and therefore follow at least in part the same trafficking pathways without necessarily interacting. 
A

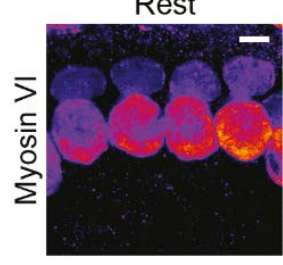

Stim $1^{\prime}$

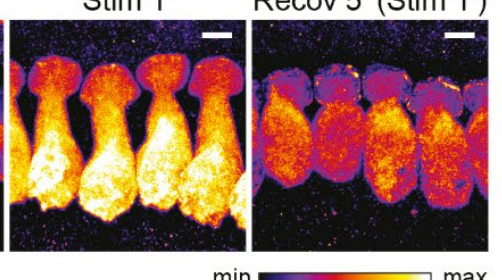

B

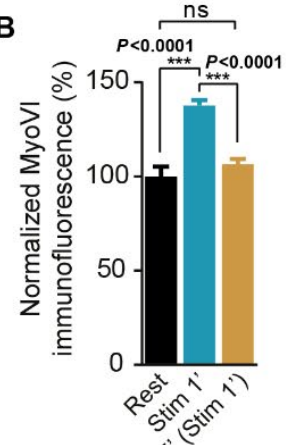

c

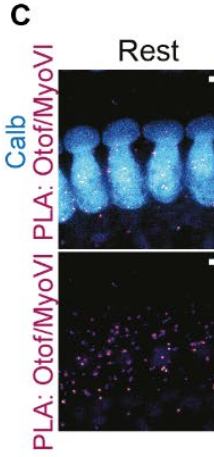

Recov 5'

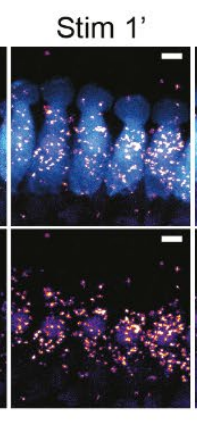

(Stim 1')
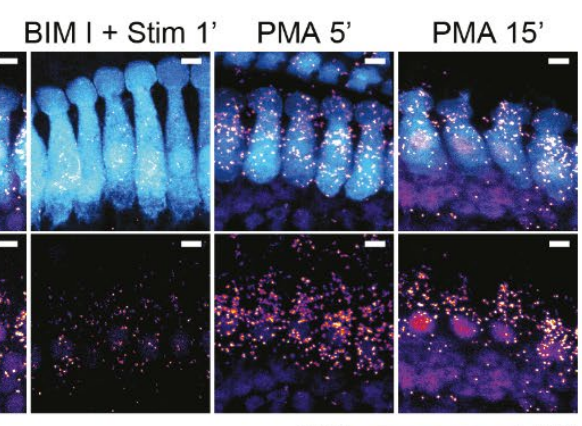

$\min \longrightarrow \max$
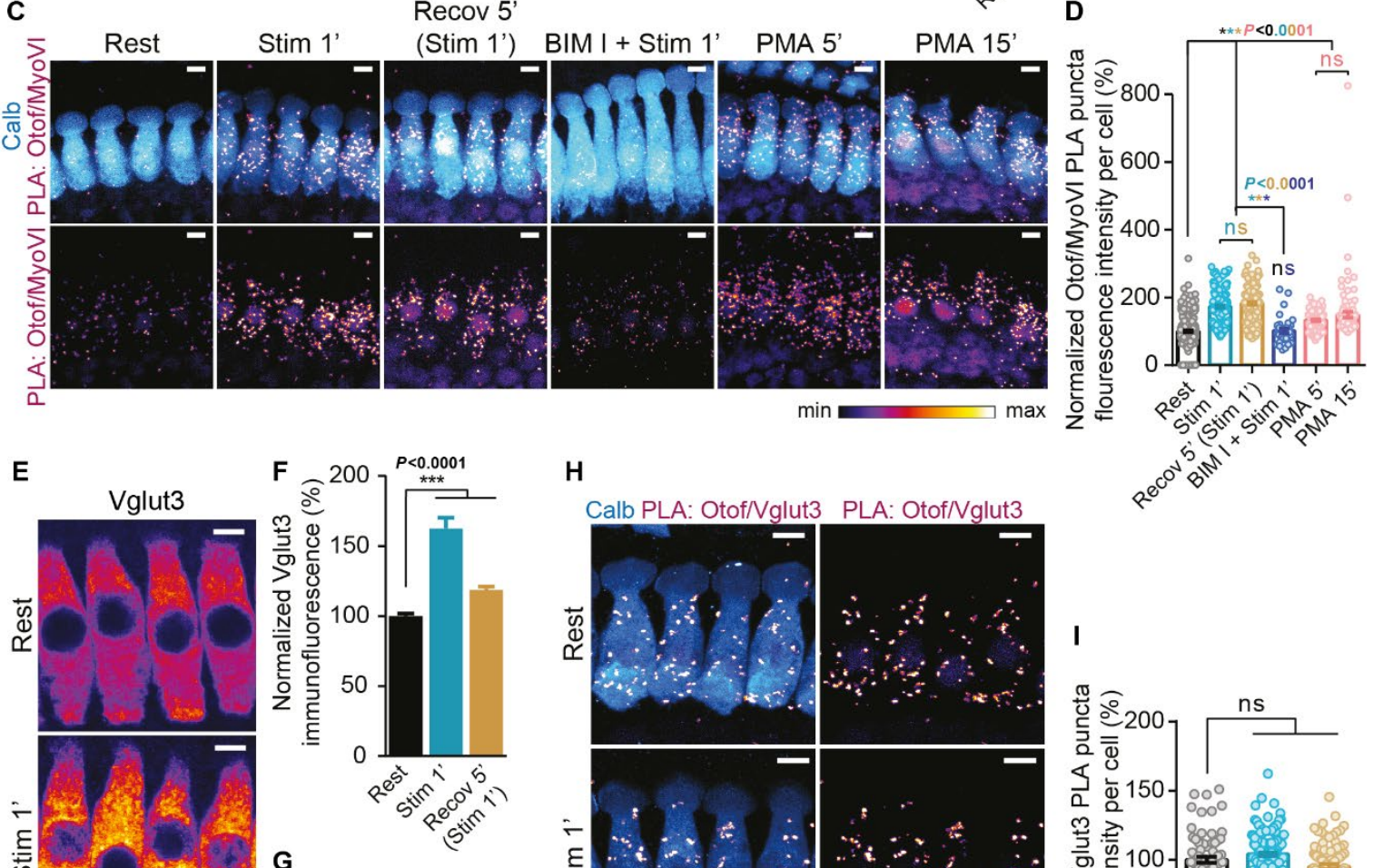

G

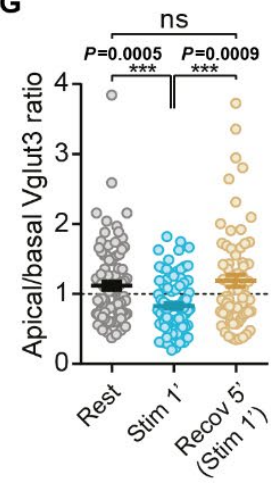

H
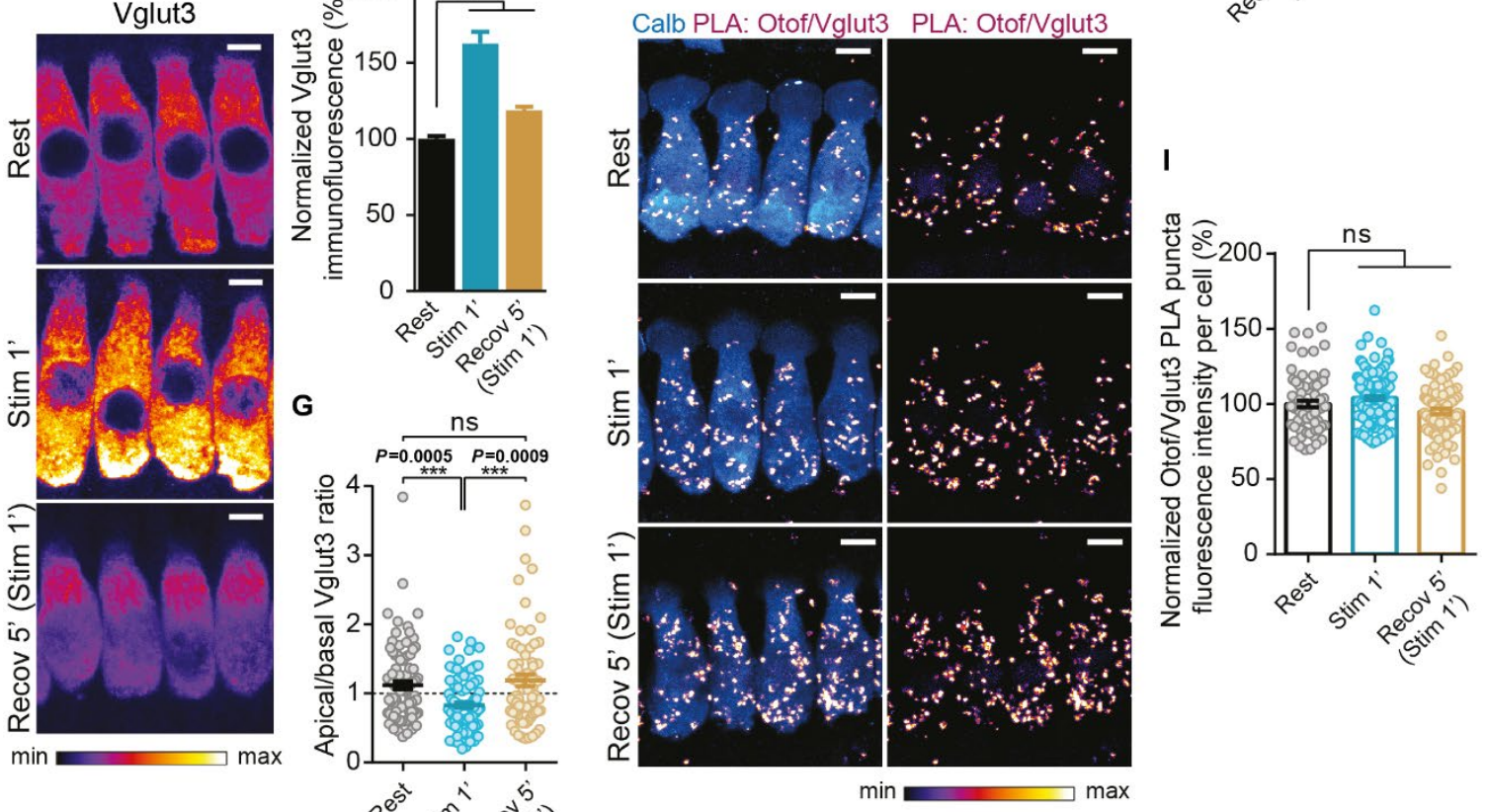

Figure 3.6. Otoferlin interacts with myosin VI, but not with Vglut3, in a PKC $\alpha$-dependent manner.

A, B Myosin VI immunofluorescence in WT P14-16 IHCs for all displayed conditions. (A) High magnification views of representative WT IHCs immunolabelled for myosin VI (intensity-coded lookup table). (B) Quantification of overall myosin VI immunofluorescence normalized to the resting condition.

C, D PLA for otoferlin and myosin VI in WT P14-16 IHCs for all indicated conditions. (C) High magnification views of representative PLAs. (D) Average otoferlin/myosinVI PLA puncta fluorescence intensity per cell, normalized to the resting condition. See control PLAs in Appendix Figure S2A and F. 
E-G Vglut3 immunofluorescence in WT P14-16 IHCs for all displayed conditions. (E) High magnification views of representative WT IHCs immunolabelled for Vglut3 (intensity-coded lookup table). (F) Quantification of overall Vglut3 immunofluorescence, normalized to the resting condition. (G) Apical/basal Vglut3 immunofluorescence (above/below nuclear midline). Number of cells in (G) also apply to $(\mathrm{F})$.

H, I PLA for otoferlin and Vglut3 in WT P14-16 IHCs for all displayed conditions. (H) High magnification views of representative PLAs. (I) Average otoferlin/Vglut3 PLA puncta fluorescence intensity per cell, normalized to the resting condition. See control PLAs in Appendix Figure S2A and E.

Data information: In (A, C, E, H), maximum intensity projections of confocal optical sections. Scale bars: $5 \mu \mathrm{m}$. In (C, H), calbindin (blue) was used as IHC marker; PLA channel is depicted with an intensity-coded lookup table with warmer colors representing higher pixel intensities. In (B, D, F-G, I), data are displayed as mean \pm s.e.m.; ns $P>0.05,{ }^{*} P \leq 0.05,{ }^{* * *} P \leq 0.001$ (Kruskal-Wallis test followed by Dunn's multiple comparison test); mean averages, sample size and statistical analysis are detailed in Appendix Table S1. In (D, G, I), individual cells are depicted with lighter colors and open symbols. Rest, resting; Stim 1', 1-minute stimulation; Stim 5', 5-minute stimulation; Recov 5' (Stim 1'), 5-minute recovery after 1-minute stimulation; BIM I + Stim 1', Incubation with BIM I prior to 1-minute stimulation; PMA 5', 5-minute incubation with PMA; PMA 15', 15-minute incubation with PMA. IHC, inner hair cell. Calb, calbindin. MyoVI, myosin VI. Otof, otoferlin. PLA, proximity ligation assay.

\section{Otoferlin interacts with calbindin and this interaction is strongly dependent on PKC $\alpha$}

In most PLA experiments we used calbindin-D28k (henceforth, calbindin) as a hair cell marker and noticed a change in calbindin immunofluorescence among the different experimental conditions (Figure 3.7A-C). Calbindin is a member of the calmodulin superfamily of $\mathrm{Ca}^{2+}-$ binding proteins and it was reported to function both as $\mathrm{Ca}^{2+}$ buffer and $\mathrm{Ca}^{2+}$ sensor (Berggard, 2002). A 1-minute high $\mathrm{K}^{+}$stimulation led to a decrease in calbindin immunofluorescence, probably due to reduced epitope accessibility (Rest: $100 \pm 1 \%, n=296$ IHCs vs. Stimulation 1': $62 \pm 2 \%, \mathrm{n}=174$ IHCs; $\left.{ }^{* * *} P<0.0001\right)$ and treatment with BIM I blocked this effect (BIM I + Stimulation 1': 99 $\pm 3 \%, n=26$ IHCs; Kruskal-Wallis test followed by Dunn's multiple comparison test; Figure 3.7B). At the same time, calbindin redistributed to the base of the IHC upon stimulation (apical/basal ratio: Rest: $1.04 \pm 0.02$ vs. Stimulation 1': $0.84 \pm 0.03$; $\left.{ }^{* * *} P<0.0001\right)$ and this effect was again blocked by BIM I (BIM I + Stimulation 1': 1.14 \pm 0.34 ; ns $P=0.8940$ vs. Rest; Kruskal-Wallis test followed by Dunn's multiple comparison test; Figure 3.7C). Calbindin seems to regain its initial location after a 5-minute recovery period (apical/basal ratio: $1.07 \pm 0.05$; ns $P=0.6380$ vs. Rest; Figure $3.7 \mathrm{C}$ ), while immunofluorescence levels remained low as for the stimulatory condition $\left(73 \pm 2 \%, \mathrm{n}=141 \mathrm{IHCs} ;{ }^{* * *} P<0.0001\right.$ vs. Rest and ${ }^{*} P=0.0152$ vs. Stimulation 1 '; Figure 3.7B). 

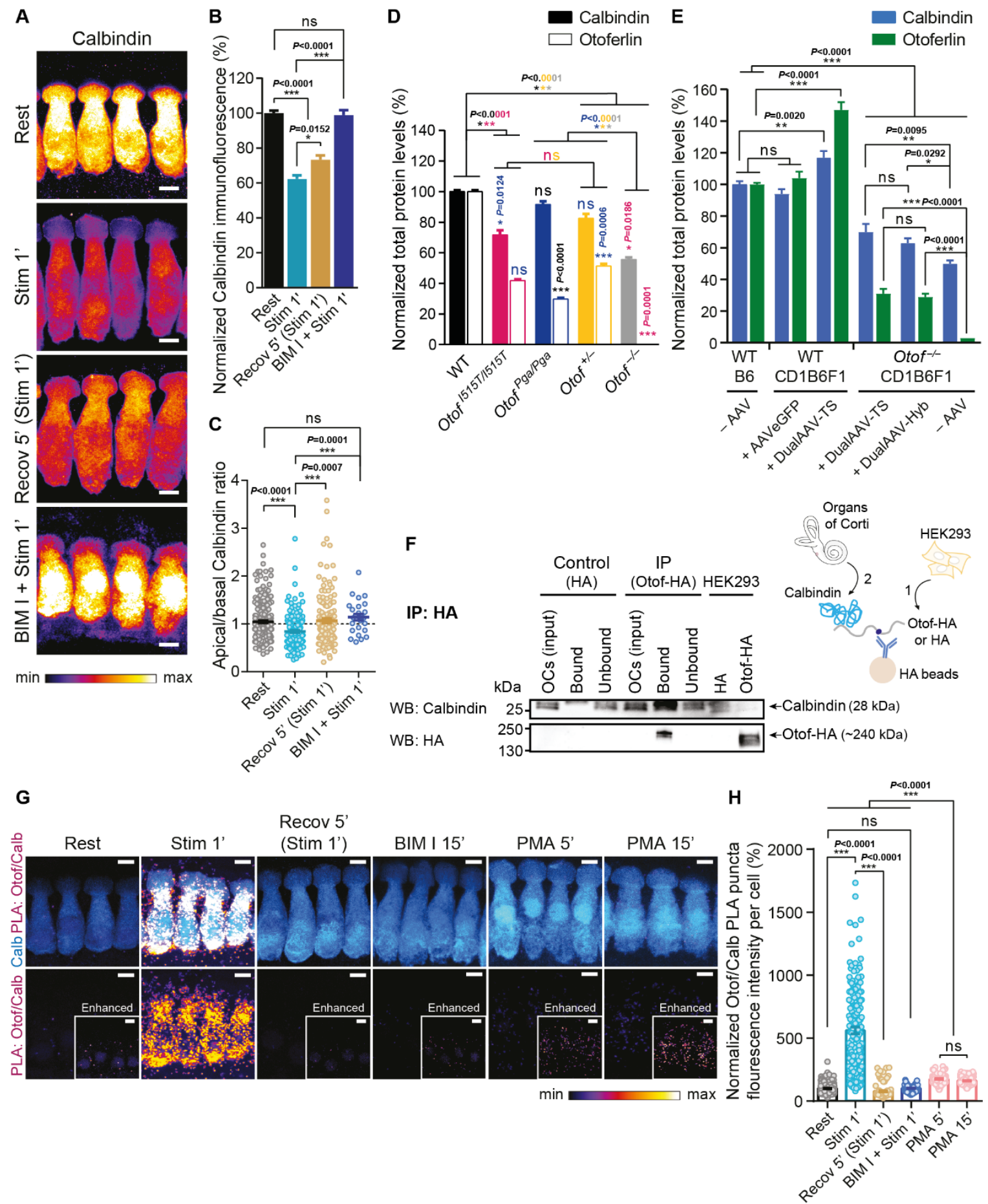

Figure 3.7. The interaction of otoferlin with calbindin is strongly dependent on PKC $\alpha$.

A-C Calbindin immunofluorescence in WT P14-16 IHCs for all indicated conditions. (A) High magnification views of representative WT IHCs immunolabelled for calbindin (intensity-coded lookup table). (B) Quantification of overall calbindin immunofluorescence. (C) Apical/basal calbindin immunofluorescence (above/below nuclear midline). Data were normalized to the resting condition. Number of cells in (C) also apply to (B).

D Average calbindin and otoferlin immunofluorescence levels in otoferlin mutant and WT IHCs (P1416). Immunofluorescence levels were normalized to WT levels for each antibody separately. See Figure EV3A for high magnification views of representative immunostainings of IHCs used for quantifications. E Average calbindin and otoferlin immunofluorescence levels in dual-AAV-transduced $\mathrm{Otof}^{\prime-}$ and WT IHCs (P23-30). Immunofluorescence levels were normalized to levels in non-transduced WT B6 IHCs 
for each antibody separately. See Figure EV3B for high magnification views of representative immunostainings of IHCs used for quantifications.

F Representative immunoblot showing results from pull-down assay from organs of Corti loaded onto anti-HA beads with previously bound otoferlin-HA expressed in HEK293T cells. Samples were probed for HA and calbindin. Right panel depicts scheme of the assay.

G, H PLA for otoferlin and calbindin performed on WT P14-16 IHCs for all indicated conditions. (G) High magnification views of representative PLAs. (D) Average otoferlin-calbindin PLA puncta fluorescence intensity per cell for all conditions, normalized to the resting condition. See control PLAs in Appendix Figure S2A and D.

Data information: In (A, G), maximum intensity projections of confocal optical sections. Scale bars: $5 \mu \mathrm{m}$. In (G), calbindin (blue) was used as IHC marker; PLA channel is depicted with an intensity-coded lookup table with warmer colors representing higher pixel intensities. In (B-E, H), data are displayed as mean \pm s.e.m.; ns $P>0.05$, ${ }^{*} P \leq 0.05,{ }^{* *} P \leq 0.01,{ }^{* * *} P \leq 0.001$ (Kruskal-Wallis test followed by Dunn's multiple comparison test); mean averages, sample size and statistical analysis are detailed in Appendix Table S1. In (C, H), individual cells are depicted with lighter colors and open symbols. Rest, resting; Stim 1', 1-minute stimulation; Stim 5', 5-minute stimulation; Recov 5' (Stim 1'), 5-minute recovery after 1-minute stimulation; BIM I + Stim 1', Incubation with BIM I prior to 1-minute stimulation; PMA 5', 5-minute incubation with PMA; PMA 15', 15-minute incubation with PMA. IHC, inner hair cell. Otof, otoferlin. Calb, calbindin. PLA, proximity ligation assay.

Calbindin levels also appear to vary among several otoferlin mutants (Figure 3.7D and Figure EV3A). In Otof ${ }^{-1}$ IHCs, calbindin levels were reduced to about $50 \%$ of WT levels (Otof ${ }^{-1}$ : $56 \pm 1 \%, \mathrm{n}=108$ IHCs vs. WT: $100 \pm 1 \%, \mathrm{n}=176$ IHCs; $\left.{ }^{* * *} P<0.0001\right)$, while IHCs of Otof ${ }^{+/}$ mice showed a reduction of about $20 \%\left(\right.$ Otof $^{+-}: 83 \pm 3 \%, \mathrm{n}=99$ IHCs; ${ }^{* * *} P=0.0002$; KruskalWallis test followed by Dunn's multiple comparison test; Figure 3.7D). For the Otof I515T/I515T mutant, carrying the temperature-sensitive p.Ile515Thr point mutation in the $\mathrm{C}_{2} \mathrm{C}$ domain of otoferlin (Strenzke et al, 2016), we found a reduction of about $25 \%$ in calbindin immunofluorescence levels when compared to WT controls (Otof ${ }^{1515 T / 1515 T}$ : 72 $\pm 3 \%, \mathrm{n}=83$ IHCs; $\left.{ }^{* * *} P<0.0001\right)$, accompanied by the previously reported reduction in otoferlin levels (Figure 3.7D). In Otof ${ }^{\text {PaalPga }}$ mutant IHCs (Pangršič et al, 2010), carrying the p.Asp1767Gly missense mutation in the $\mathrm{C}_{2} \mathrm{~F}$ domain, there were no evident changes in calbindin levels when compared to WT IHCs (Otof Pgal/ga: $92 \pm 2 \%, \mathrm{n}=76$ IHCs; ns $P=0.5900$; Kruskal-Wallis test followed by Dunn's multiple comparison test), although otoferlin levels are slightly lower in this mutant by comparison to Otof ${ }^{1515 T / 1515 T}$ IHCs (Figure 3.7D). In our recent study where we partially rescued hearing in $\mathrm{Otof}^{-1-}$ mice by reintroducing otoferlin in IHCs via dual-AAV approaches (Al-Moyed et al, 2019), we quantified calbindin immunofluorescence levels alongside otoferlin levels (Figure 3.7E and Figure EV3B). Reintroduction of otoferlin led to an increase in calbindin levels not only in $\mathrm{Otof}^{-/} \mathrm{IHCs}$ (untreated Otof ${ }^{-/} \mathrm{CD} 1 \mathrm{~B} 6 \mathrm{~F} 1: 50 \pm 2 \%$, $\mathrm{n}=142$ IHCs; Otof ${ }^{-/}$CD1B6F1+DualAAV-Hybrid: 63 $\pm 3 \%, \mathrm{n}=64$ IHCs; Otof ${ }^{-/}$ CD1B6F1+DualAAV-Trans-splicing: $70 \pm 5 \%, \mathrm{n}=13$ IHCs), but also in wild-type IHCs (untreated WTB6: $100 \pm 2 \%, \quad \mathrm{n}=276$ IHCs; WTCD1B6F1+DualAAV-Trans-splicing: $117 \pm 4 \%, n=62$ IHCs; Figure 3.7E). 
A
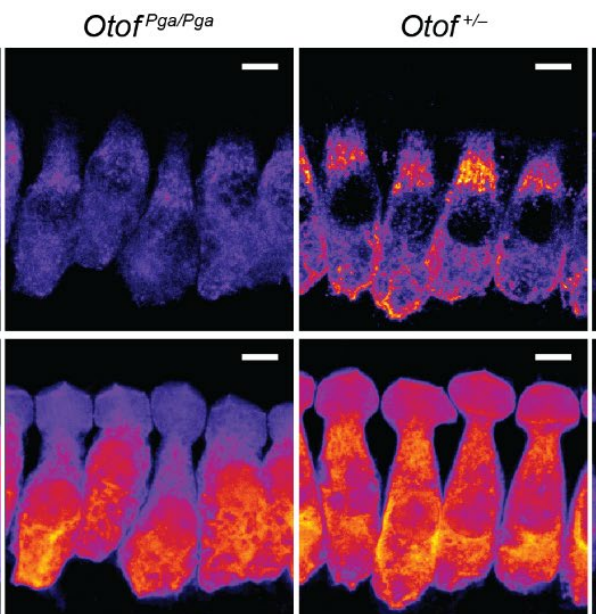

Otof $^{-1-}$
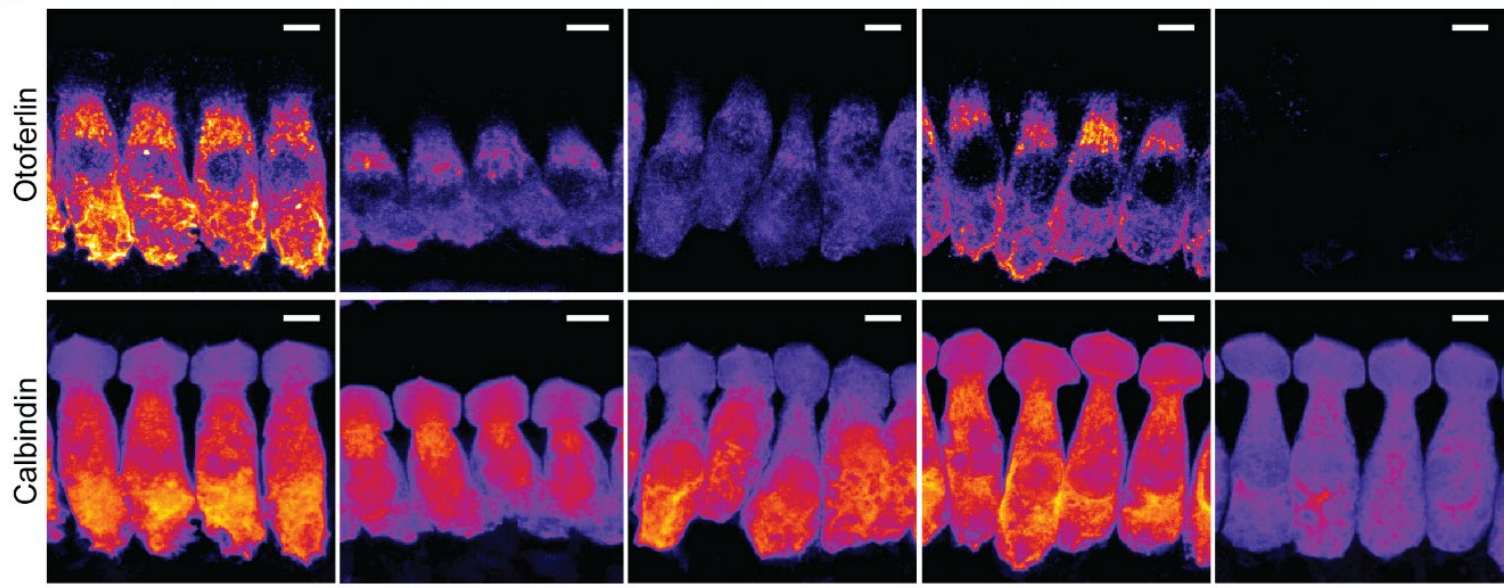

$\min \underset{\max }{\longrightarrow}$

B
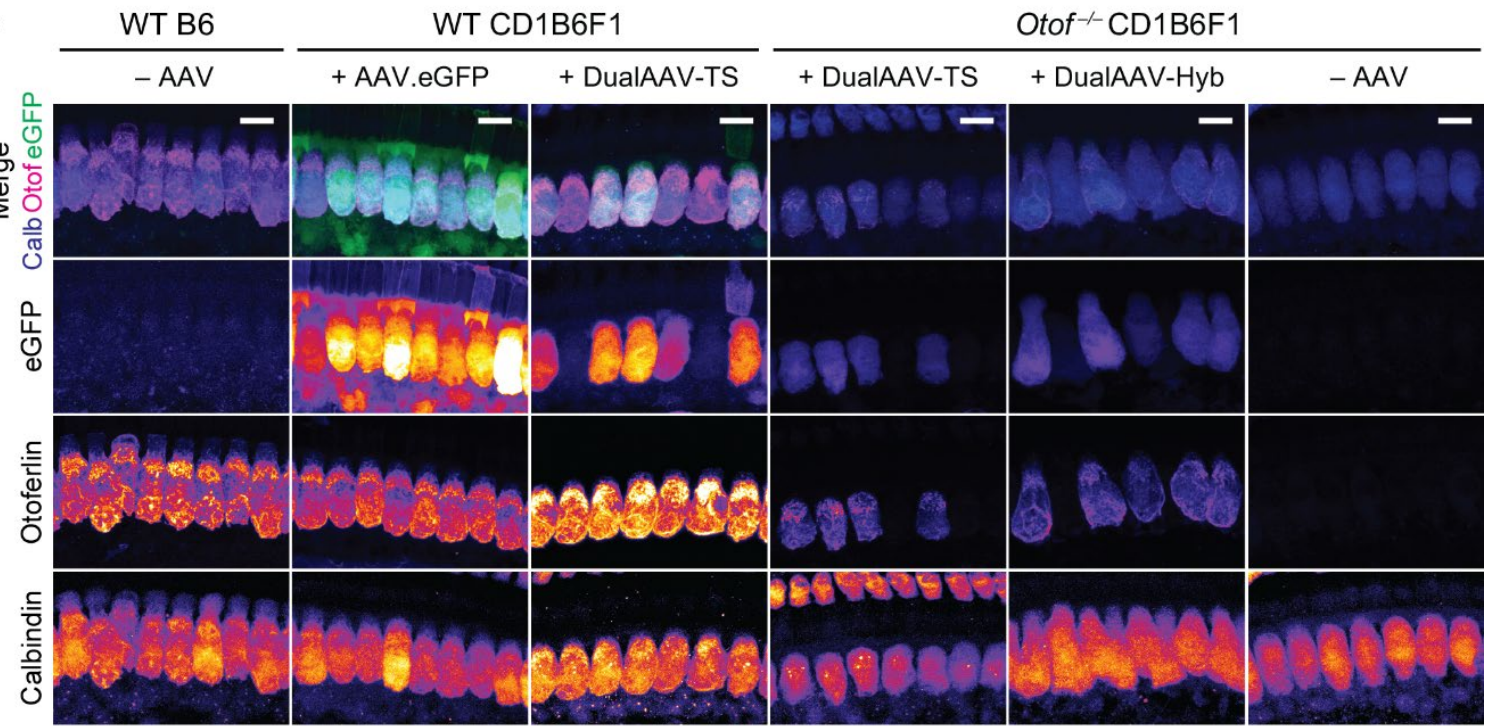

$\min$

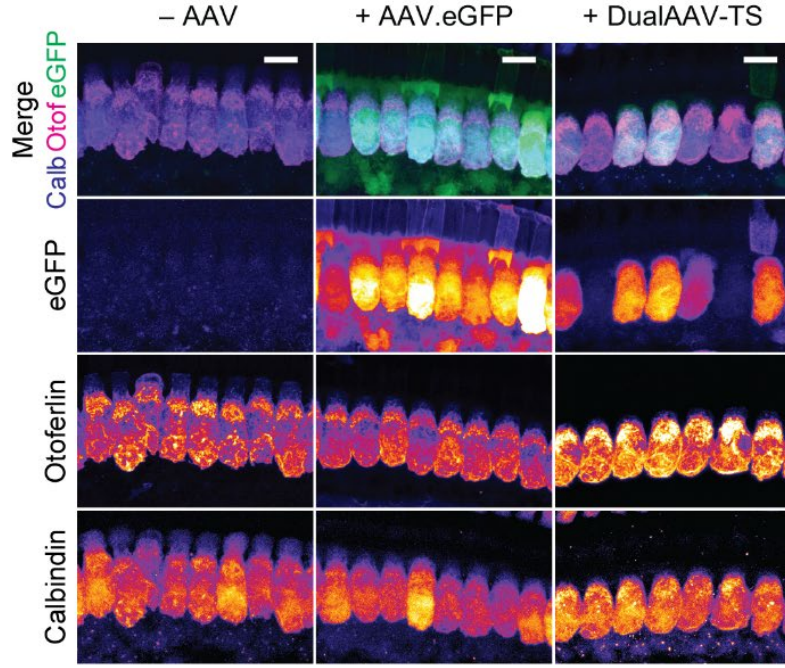

$\min$

Figure EV3. Calbindin and otoferlin immunofluorescence in different otoferlin mutants and in dual-AAVtransduced $\mathrm{Otof}^{--}$and WT IHCs.

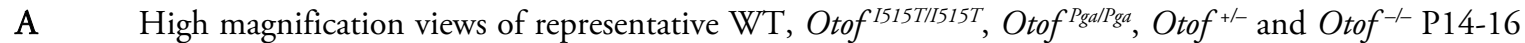
IHCs immunolabeled for calbindin and otoferlin, used for quantification of calbindin levels in Figure 3.7D. Individual calbindin and otoferlin channels are depicted separately with an intensity-coded lookup table with warmer colors representing higher pixel intensities.

B High magnification views of dual-AAV-TS (P26) and dual-AAV-Hyb (P26) transduced CD1B6F1Otof $^{-1}$ IHCs compared to AAV2/6.eGFP transduced WT CD1B6F1 (P28) and non-injected WT B6 (P27) IHCs, used for quantification of calbindin levels in Figure 3.7E. Successful virus transduction was monitored via eGFP immunofluorescence (green). Organs of Corti were immunolabeled against otoferlin (magenta) and calbindin (blue). Individual eGFP, otoferlin, and calbindin channels are depicted separately with an intensity-coded lookup table with warmer colors representing higher pixel intensities.

Data information: In (A-B), maximum intensity projections of optical confocal sections. Scale bars: $5 \mu \mathrm{m}(\mathrm{A})$, $10 \mu \mathrm{m}$ (B). IHC, inner hair cell. Calb, calbindin. Otof, otoferlin. 
64 | Chapter 3

To explore a possible interaction of otoferlin and calbindin, we repeated the otoferlin-HA pulldowns described before but this time we immunoblotted for calbindin. A strong band of $\sim 28$ $\mathrm{kDa}$ in the eluate indicates a direct interaction of otoferlin and calbindin in vitro (Figure 3.7F).

We then assessed a possible interaction of otoferlin and calbindin and its potential dependency on PKC $\alpha$ activation in IHCs of explanted organs of Corti (Figure 3.7G-H). In resting conditions, we found few PLA puncta throughout the IHCs. A 1-minute high $\mathrm{K}^{+}$stimulation led to a $>5$-fold increase in PLA signal (Rest: 100 $\pm 2 \%, n=327$ IHCs vs. Stimulation 1': $560 \pm 26 \%, \mathrm{n}=168$ IHCs; $\left.{ }^{* * *} P<0.0001\right)$. After a 5 -minute recovery period, the PLA signal dropped to values lower than those of the resting condition (Recovery 5': 77 $\pm 5 \%, \mathrm{n}=107$ IHCs; ${ }^{* * *} P<0.0001$ vs. Rest). Treatment with the PKC inhibitor BIM I fully blocked the stimulationinduced increase in PLA signal (BIM I + Stimulation 1': 101 $\pm 3 \%, \mathrm{n}=98$ IHCs; $\mathrm{ns} P>0.9999$ vs. Rest). Incubation with PMA led to an increase in PLA signal, though not as pronounced as for high $\mathrm{K}^{+}$stimulation (PMA 5’: 175 $\pm 4 \%, \mathrm{n}=114$ IHCs and PMA 15’: 161 $\pm 3 \%, \mathrm{n}=127$ IHCs; ${ }^{* * *} P<0.0001$ vs. Rest and ${ }^{* * *} P<0.0001$ vs. Stimulation $1^{\prime}$; Kruskal-Wallis test followed by Dunn's multiple comparison test). Thus, otoferlin and calbindin interact in IHCs in a strong activity- and PKC $\alpha$-dependent manner.

A PLA between PKC $\alpha$ and calbindin in the same conditions (Figure EV4) resulted in an increased PLA signal after stimulation (Rest: 100 $\pm 6 \%, n=75$ IHCs vs. Stimulation 1': 158 $\pm 5 \%$, $\mathrm{n}=94 \mathrm{IHCs} ;{ }^{* * *} P<0.0001$ ), yet not as demarked as the increase observed for the PLAs between otoferlin and PKC $\alpha$ and between otoferlin and calbindin. This points toward an indirect interaction between calbindin and PKC $\alpha$ via a scaffolding protein, likely otoferlin. It is also conceivable that PKC $\alpha$, otoferlin and calbindin are part of the same complex at least at some point during strong stimulation, with $\mathrm{PKC}$ and calbindin binding to distinct regions of otoferlin. 
A

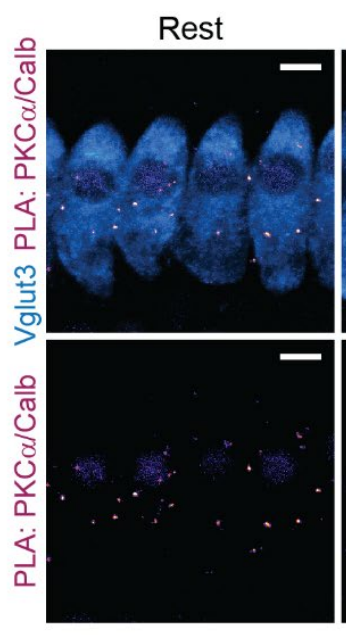

Stim $1^{\prime}$

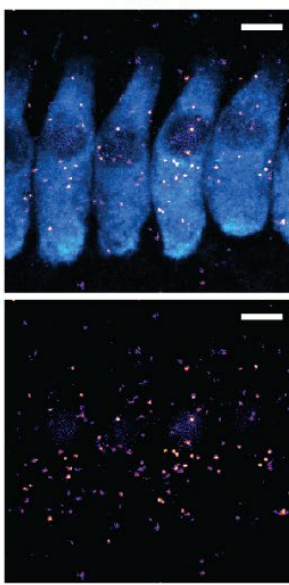

Recov 5' (Stim 1')

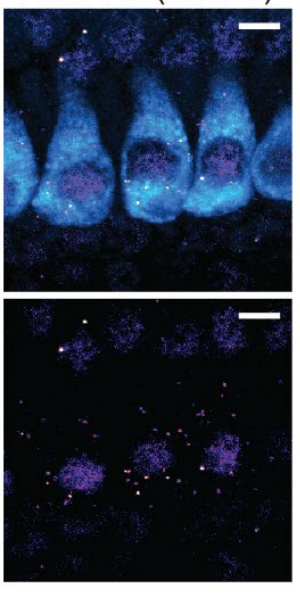

$\min$
B

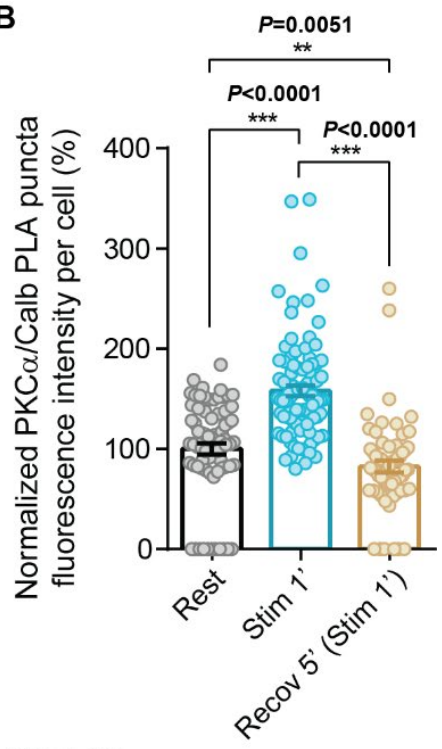

C

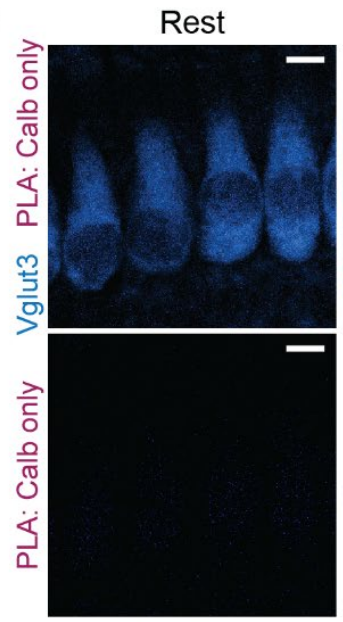

Stim 1'

Recov 5' (Stim 1')

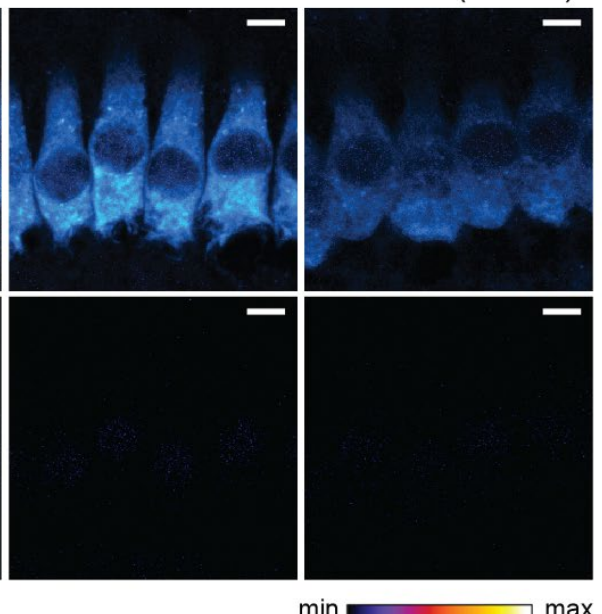

Figure EV4. Weak PLA signal between PKC $\alpha$ and calbindin points toward an indirect interaction of the two proteins via scaffolding proteins.

A-B PLA for PKC $\alpha$ and calbindin performed on WT P15-16 IHCs for the indicated conditions. (A) High magnification views of representative PLAs. (B) Average PKCa/calbindin PLA puncta fluorescence intensity per cell, normalized to the resting condition. Individual cells are depicted with lighter colors and open symbols.

C High magnification views of representative control PLA performed with calbindin primary antibody only. See control PLA with PKC $\alpha$ antibody only in Appendix Figure S2C.

Data information: In (A, C), maximum intensity projections of confocal optical sections. Scale bars: $5 \mu \mathrm{m}$. Vglut3 (blue) was used as IHC marker. The PLA channel is depicted with an intensity-coded lookup table with warmer colors representing higher pixel intensities. Rest, resting; Stim 1', 1-minute stimulation; Recov 5' (Stim 1'), 5minute recovery after 1-minute stimulation. Calb, calbindin. 


\subsubsection{Discussion}

Inner hair cells exhibit an extraordinarily high rate of synaptic vesicle turnover. Both exocytosis and endocytosis are known to be regulated by $\mathrm{Ca}^{2+}$ (Beutner et al, 2001). In this study, we found two $\mathrm{Ca}^{2+}$-binding proteins, PKC $\alpha$ and calbindin, to interact with otoferlin, thereby forming a $\mathrm{Ca}^{2+}$-dependent signaling complex that likely regulates different modes of endocytosis at IHC synapses.

Upon high $\mathrm{K}^{+}$exposure leading to IHC depolarization, $\mathrm{Ca}^{2+}$ influx through voltage-gated $\mathrm{Ca}^{2+}$ channels triggers exocytosis, but also activates $\mathrm{Ca}^{2+}$-dependent kinases like PKC and CaMKII (Meese et al, 2017 and this study). Several proteins located next to the $\mathrm{Ca}^{2+}$ sources bind $\mathrm{Ca}^{2+}$, e.g. the proposed $\mathrm{Ca}^{2+}$ sensor for exocytosis at this synapse, otoferlin, as well as $\mathrm{Ca}^{2+}$ buffer proteins like calbindin, parvalbumin and calretinin (Pangršič et al, 2015). Among these, regulatory roles have so far been attributed to calbindin only (Berggard, 2002). In this study we found that PKC activation in IHCs, either pharmacologically or upon high $\mathrm{K}^{+}$stimulation, triggers the interaction of PKC with otoferlin, resulting in the phosphorylation of otoferlin at S158, S775, S1169, S1224 and S1436 residues. These post-translational modifications might enable otoferlin to interact with other proteins, like myosin VI and calbindin. Pharmacological activation of PKC $\alpha$ without intracellular $\mathrm{Ca}^{2+}$ elevation also induced the interaction of otoferlin with calbindin and myosin VI, although not as effectively as by cell depolarization which triggers $\mathrm{Ca}^{2+}$ influx, indicating that $\mathrm{Ca}^{2+}$ binding to either one or both proteins strongly promotes the interaction. Since PKC inhibition before high $\mathrm{K}^{+}$exposure abolished the association of otoferlin with calbindin and a direct interaction of PKC $\alpha$ and calbindin is rather unlikely, the phosphorylation of otoferlin by PKC $\alpha$ seems to be a prerequisite for the otoferlincalbindin interaction. It is noteworthy that the increase in PLA signal for calbindin and PKC $\alpha$ was much weaker than for the other combinations under the same stimulatory conditions, suggesting either that calbindin and PKC $\alpha$ bind to distal parts of otoferlin, or PKC $\alpha$ dissociates from the complex after calbindin binds to phosphorylated otoferlin.

The activation of PKC $\alpha$ upon high $\mathrm{K}^{+}$stimulation was characterized by accumulations of PKC $\alpha$ and otoferlin in common structures near the active zones. A closer observation of the subcellular location of the interaction revealed clearly rendered fluorescent hotspots close to the synaptic ribbons. These structures were revealed to be larger than synaptic vesicles and resemble recycling endosomes described elsewhere (Kamin et al, 2014; Revelo et al, 2014; Watanabe et al, 2014; Jung et al, 2015a). In an earlier study, we examined IHCs at the ultrastructural level and we found otoferlin immunogold labelling to localize to membranous compartments of $>50 \mathrm{~nm}$ diameter close to active zones, which were clearly larger than synaptic vesicles of $-40 \mathrm{~nm}$. Many of the otoferlin-immunogold-labelled structures had a clathrincoated pit at its edge, indicating these structures are most likely endosomal recycling compartments (Strenzke et al, 2016, Figure 7I,F,G). In addition, some otoferlin-labelled endocytic structures resembled ultrafast endocytic compartments (Strenzke et al, 2016, Figure 
7I, F, G), which are located laterally to active zones and are about four times the size of synaptic vesicles in hippocampal synapses (Watanabe $e t$ al, 2013). Since ultrafast endocytosis requires a plasma membrane excess at active zones, which occurs only after strong exocytosis (Watanabe et al, 2013), and lower exocytosis rates rather induce clathrin mediated endocytosis (Kamin et al, 2014; Revelo et al, 2014) it is noteworthy that weak stimulation paradigms did not lead to PKC $\alpha$ immunofluorescence clustering in IHCs (Figure EV2A). Notably, in central nervous system synapses PKC was shown to be essential for the trafficking of synaptotagmin IX to endocytic recycling compartments (Haberman et al, 2005), but also seems to be involved in endocytic processes in general (Alvi et al, 2007). We thus propose that the structures where otoferlin and PKC interact in IHCs are most likely endocytic recycling compartments.

The nature of proteins which we found to interact with otoferlin in an activity-dependent and strongly PKC $\alpha$-dependent manner supports our hypothesis that PKC $\alpha$ might be involved in regulating different modes of endocytosis. Upon high $\mathrm{K}^{+}$IHC stimulation or treatment with a PKC activator, we observed an increase in PLA signal for the previously reported interaction of otoferlin and myosin VI (Roux et al, 2009; Heidrych et al, 2009). Myosin VI, like other myosin motors, interacts with filamentous actin (F-actin) generating the force that propels the sliding of these filaments and moves along them, thereby regulating the dynamics of the actin cytoskeleton and affecting the transport of cellular components (reviewed in Kneussel \& Wagner, 2013). It was also reported that F-actin seems to control otoferlin-dependent exocytosis in auditory IHCs (Vincent et al, 2015), where it forms dense cage-shaped structures beneath the synaptic ribbon thereby maintaining a tight spatial organization of calcium channels at the active zones. Additionally, the authors show that F-actin colocalizes with otoferlin at the basal region of the IHC, predicting a physical association between them. Moreover, the unique myosin VI motor is involved in the early endocytic pathway, where it is required for cargo sorting (Tumbarello et al, 2013), so it seems plausible that both myosin VI and F-actin in association with otoferlin are involved in cellular trafficking processes in a PKCdependent manner, which might include trafficking of endosomal compartments in IHCs.

What might be the role of calbindin in this complex? The finding that calbindin immunofluorescence is strongly reduced in Otof ${ }^{1515 T / 1515 T}$ but not in Otof ${ }^{\text {PgalPga }}$ IHCs seems contradictory in the first place. Yet, a potential explanation might be that these mutations differentially impair distinct cellular processes, like vesicle replenishment (proposed for Otof $P_{g a / P g a}$ ) and vesicle reformation from endocytic recycling compartments (ascribed to Otof

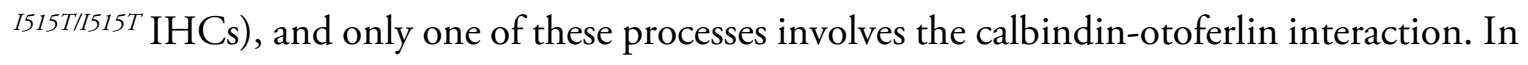
addition, a knock-out of calbindin does not affect hearing or susceptibility to noise, at least regarding threshold shifts (Airaksinen et al, 2000). Although a role in noise-induced synaptopathy cannot be ruled out, the short timescale of the interaction, growing weaker between 1 and 5-minute depolarizations, makes it unlikely that calbindin acts in processes that need to last from minutes to hours, such as affecting the susceptibility to noise. Similarly, triple 
knock-out mice of calbindin, parvalbumin and calretinin $\left(\mathrm{Ca}^{2+}\right.$ buffer $\left.\mathrm{TKO}\right)$ showed remarkably low impact on hearing (Pangršič et al, 2015). In patch-clamp recordings from $\mathrm{Ca}^{2+}$ buffer TKO IHCs, exocytosis upon short stimuli (reflecting the fusion of the readily releasable pool of vesicles) remained wild-type-like; hence, an involvement of the $\mathrm{Ca}^{2+}$ buffer proteins in vesicle fusion seems unlikely. However, for longer stimuli (100-ms and 200-ms-long depolarizations to $-17 \mathrm{mV})$, the change in plasma membrane capacitance $\left(\Delta \mathrm{C}_{\mathrm{m}}\right)$ was larger in $\mathrm{Ca}^{2+}$ buffer TKO than in wild-type control IHCs (Pangršič et al, 2015, Figure 3C). Substitution of endogenous buffers with variable concentrations of the synthetic $\mathrm{Ca}^{2+}$ buffers EGTA or BAPTA could not accurately restore the $\mathrm{C}_{\mathrm{m}}$ changes in response to fast and sustained stimuli to wild-type values, indicating that at least one of the $\mathrm{Ca}^{2+}$ buffer proteins might fulfill an additional function over simple $\mathrm{Ca}^{2+}$ buffering. At the time, the larger $\Delta \mathrm{C}_{\mathrm{m}}$ obtained for $\mathrm{Ca}^{2+}$ buffer TKO IHCs in response to longer depolarizations was presumed to reflect an increase in exocytosis, which, nonetheless, did not trigger more action potentials in postsynaptic neurons, and this apparent increase in exocytosis was then attributed to extrasynaptic vesicle fusion. Yet, 200-ms-long stimulations resulted in a $\Delta \mathrm{C}_{\mathrm{m}}$ of $360 \mathrm{fF}$ in $\mathrm{Ca}^{2+}$ buffer TKO IHCs vs. $116 \mathrm{fF}$ in wild-type IHCs (at $2 \mathrm{mM}\left[\mathrm{Ca}^{2+}\right]_{\mathrm{e}}$ ), implying that extrasynaptic exocytosis would need to occur at double the rate of synaptic exocytosis if this were the only explanation. However, corresponding amounts of extrasynaptic synaptic vesicles were never found in EM ultrastructure images, and particularly the ribbon is presumed to assist in vesicle reformation and resupply (Jung et al, 2015a; Pangrsic \& Vogl, 2018; Jean et al, 2018). Instead, we favor the hypothesis that ultrafast endocytosis, occurring in wild-type but absent in $\mathrm{Ca}^{2+}$ buffer TKO IHCs, might explain a major part of the difference in $\Delta \mathrm{C}_{\mathrm{m}}$ for 100 -ms and 200ms stimulations. This mode of endocytosis was first proposed by Watanabe and collaborators (Watanabe et al, 2013). The authors stimulated hippocampal neurons expressing channelrhodopsin with a short light pulse and fixed the tissue within few milliseconds by highpressure quick freezing ("flash-and-freeze"). Ultrastructural analysis revealed membrane invaginations next to active zones, which were detached from the plasma membrane between 50 and $100 \mathrm{~ms}$ of stimulation. In $\mathrm{C}_{\mathrm{m}}$ recordings, membrane invaginations do not lead to a reduction of cellular capacitance, but once the compartments become constricted and are further internalized, the plasma membrane surface area, and proportionally to it the $\mathrm{C}_{\mathrm{m}}$, decrease. In the recordings of Pangršič et al (2015), $\Delta \mathrm{C}_{\mathrm{m}}$ from $\mathrm{Ca}^{2+}$ buffer TKO IHCs was larger than in wild-type IHCs, but only from 100 -ms stimulations onwards $\left(-140 \mathrm{fF}\right.$ in $\mathrm{Ca}^{2+}$ buffer TKO IHCs vs. $-60 \mathrm{fF}$ in wild-type IHCs for 100 -ms depolarizations at $2 \mathrm{mM}\left[\mathrm{Ca}^{2+}\right]_{\mathrm{e}}$ ). This could be interpreted that at least one of the $\mathrm{Ca}^{2+}$ buffer proteins might be required for ultrafast endocytosis.

In a follow-up study, Watanabe and collaborators found that ultrafast endocytosis depends on actin polymerization (Watanabe et al, 2014). When actin polymerization was inhibited with latrunculin $\mathrm{A}$, the authors found a strong reduction in ultrafast endocytosis, again revealed by flash-and-freeze and EM analysis. In different studies aiming at elucidating the role of actin 
polymerization in IHC synaptic function (Vincent et al, 2015; Guillet et al, 2016), latrunculin A was used during $\mathrm{C}_{\mathrm{m}}$ recordings of IHCs. Again, $\Delta \mathrm{C}_{\mathrm{m}}$ increased more in latrunculin A-treated IHCs both for whole-cell patch clamp recordings and flash photolysis of caged $\mathrm{Ca}^{2+}$. The authors interpreted this as facilitation of exocytosis by reduction of actin filament-based diffusion barriers and proposed a role for F-actin in controlling the diffusion rate of the synaptic vesicles to the sites of release in IHCs. However, $\mathrm{Ca}^{2+}$ uncaging experiments in Vincent et al (2015) show that there is hardly any difference in exocytic rates in the first $50 \mathrm{~ms}$ both in presence and absence of latrunculin A (Vincent et al, 2015, Figure 2D), indicating that in this experimental setting the diffusion of vesicles to the sites of release was comparable. Differences in kinetics were rather registered between 50 to $100 \mathrm{~ms}$ after the flash (faster increase in $\mathrm{C}_{\mathrm{m}}$ for latrunculin A-treated IHCs), which is coherent with the proposed timescale for ultrafast endocytosis. We thus favor the hypothesis that the increased $\Delta \mathrm{C}_{\mathrm{m}}$ in presence of latrunculin $\mathrm{A}$, both for step depolarizations and flash photolysis, reflects absence of ultrafast endocytosis. In Guillet et al (2016), $\Delta \mathrm{C}_{\mathrm{m}}$ was significantly larger in presence of both actin polymerization inhibitors used, but only for 20 -ms-long stimulations $(-120 \mathrm{fF}$ with latrunculin A vs. $-20 \mathrm{fF}$ without, $\left.2 \mathrm{mM}\left[\mathrm{Ca}^{2+}\right]_{\mathrm{e}}\right)$. For longer stimuli, two actin-dependent processes might be impaired that differentially affect $\Delta \mathrm{C}_{\mathrm{m}}$ : the impairment of ultrafast endocytosis, increasing $\Delta \mathrm{C}_{\mathrm{m}}$, and a reduction in vesicle replenishment, reducing $\Delta \mathrm{C}_{\mathrm{m}}$ in comparison to untreated cells. Both effects combined might thus have resulted in non-significantly different $\Delta \mathrm{C}_{\mathrm{m}}$ for 50 to 100-ms-long depolarizations.

More recently, Tertrais and collaborators blocked the fission of endocytic invaginations with the dynamin blocker dyngo-4a and observed an increase in $\Delta \mathrm{C}_{\mathrm{m}}$ over control values $(-50 \mathrm{fF}$ vs. $-35 \mathrm{fF}$ for a train of five consecutive $20-\mathrm{ms}$ depolarizations, $\left.5 \mathrm{mM}\left[\mathrm{Ca}^{2+}\right]_{\mathrm{e}}\right)($ Tertrais et al, 2019). This would be in agreement with the assumption that IHC synapses compensate the extraordinary release rates of synaptic vesicles by ultrafast endocytosis. For endocytosis triggered by flash photolysis of caged $\mathrm{Ca}^{2+}$ in IHCs, time constants of $10 \mathrm{~ms}$ for $\Delta \mathrm{C}_{\mathrm{m}}$ were found, which would be even faster than reported for ultrafast endocytosis at hippocampal synapses. Although this might be a plausible scenario that would explain how this synapse compensates the extraordinarily high rates of exocytosis and compares to the effect found after 20 ms stimulation in Guillet et al (2016), the triggering of vesicle fusion by $\mathrm{Ca}^{2+}$ uncaging is a rather unphysiological strong stimulus. It increases the cellular surface by $>1 \mathrm{pF}$, which would require 22000 synaptic vesicles (of $45 \mathrm{aF}$ each) per $\mathrm{pF}$ (Neef et al, 2007a) to fuse with the plasma membrane and might induce endocytic mechanisms that do not typically occur in more physiological conditions. Since $C_{m}$ recordings only reveal the sum of endocytic and exocytic events, it will be important to confirm this remarkably ultrafast kinetics of endocytosis by flashand-freeze experiments in IHCs.

What other molecular players could be involved in ultrafast endocytosis at IHC synapses? Endophilin A was first attributed to play a role in fast bulk endocytosis, but with slower kinetics 
than that of ultrafast endocytosis (Watanabe \& Boucrot, 2017). More recently, endophilin A and synaptojanin were found to accelerate ultrafast endocytosis at hippocampal synapses (Watanabe et al, 2018). In $\mathrm{C}_{\mathrm{m}}$ recordings of endophilin A knock-out IHCs no apparent increase in $\Delta \mathrm{C}_{\mathrm{m}}$ was observed (Kroll et al, 2019), seemingly arguing against an involvement of endophilin A in ultrafast endocytosis at this synapse. However, chronic impairment of endocytosis and vesicle reformation will inevitably affect vesicle replenishment. Since both endophilin $\mathrm{A}$ and synaptojanin are known to be required not only for fission of bulk endosomes but also for clathrin uncoating of recycling vesicles, inhibition of synaptic vesicle recycling might act more strongly on synaptic function in $C_{m}$ recordings than the slowing down of ultrafast endocytosis.

In conclusion, we showed that $\mathrm{Ca}^{2+}$ influx activates $\mathrm{PKC} \alpha$, which phosphorylates otoferlin, enabling it to interact with calbindin and myosin VI. We propose that the association of these proteins constitutes a molecular switch with the assembly of the otoferlin-calbindin complex being required for ultrafast endocytosis in IHCs.

\subsubsection{Materials and Methods}

\section{Study approval}

Animal handling and experiments complied with national animal care guidelines and were approved by the board for animal welfare of the University of Göttingen and the animal welfare office of the state of Lower Saxony, Germany.

\section{Animals}

Wild-type C57BL/6J (B6), Otof I515T/I515T (Strenzke et al, 2016), Otof Pga/Iga (Pangršič et al, 2010) and Otof $^{-1-}$ (Reisinger et al, 2011) mice of either gender were used. For otoferlin rescue experiments, $\mathrm{CD} 1 \times \mathrm{xC} 57 \mathrm{BL} / 6 \mathrm{~N}-\mathrm{F} 1 \quad(\mathrm{CD} 1 \mathrm{~B} 6 \mathrm{~F} 1)$ Otof ${ }^{-/}$and control wild-type CD1xC57BL/6N-F1 (CD1B6F1) or wild-type C57BL/6J (B6) mice were used, as previously described (Al-Moyed et al, 2019). The mice were housed in social groups in individually ventilated cage (IVC) racks in a specific pathogen-free facility with free access to food and water and 12-h/12-h light/dark cycles. 


\section{Constructs}

RNA isolation and cDNA synthesis from mouse organs of Corti (OCs) were carried out as described previously (Al-Moyed et al, 2019). To generate eGFP-mPKC $\alpha$, protein kinase C $\alpha$ cDNA (NM_011101.3) was amplified from the organ of Corti cDNA and subcloned into pEGFP-C2. The mCherry-P2A-mOtof-HA vector contains mCherry, a P2A peptide sequence inducing ribosome skipping (Kim et al, 2011), the mouse organ of Corti otoferlin coding sequence (CDS) (transcript variant 4, KX060996; NM_001313767) (Strenzke et al, 2016) and a hemagglutinin (HA) epitope tag (YPYDVPDYA) introduced in a region where no deleterious mutations in otoferlin were reported. To generate mCherry-P2A-HA, the HAtagged otoferlin CDS in mCherry-P2A-mOtof-HA was replaced by an HA tag.

\section{Co-immunoprecipitation in HEK cells}

HEK293T cells were plated at a density of $1 \times 10^{6}$ cells per $10 \mathrm{~cm}$ dish and transfected Lipofectamine 3000 (\#L3000015, Thermo Fisher Scientific) 24h post-seeding. For GFP immunoprecipitation, cells were transfected with mCherry-P2A-mOtof-HA and eGFP$\mathrm{mPKC} \alpha$ or mCherry-P2A-mOtof-HA only (control). For HA immunoprecipitation, cells were transfected with mCherry-P2A-mOtof-HA and eGFP-mPKC $\alpha$ or eGFP-mPKC $\alpha$ only (control). Cells were harvested $72 \mathrm{~h}$ post-transfection by washing three times in PBS (137 mM $\mathrm{NaCl}, 2.7 \mathrm{mM} \mathrm{KCl}$ and $10 \mathrm{mM}$ phosphate buffer solution, $\mathrm{pH} 7.4$ ), and lysed in NP-40 lysis buffer supplemented with protease inhibitors $(10 \mathrm{mM}$ Tris- $\mathrm{HCl} \mathrm{pH} 7.5,150 \mathrm{mM} \mathrm{NaCl}, 0.5$ mM EDTA pH 8.0, 0.5\% NP-40, protease inhibitors (\#4693132001, Roche, cOmplete ${ }^{\mathrm{TM}}$, EDTA-free Protease Inhibitor Cocktail)) by pipetting extensively for $1 \mathrm{~h}$ on ice and centrifuged at $500 \mathrm{x} \mathrm{g}, 4{ }^{\circ} \mathrm{C}$ for $5 \mathrm{~min}$ to remove cell debris. The lysates were mixed with $25 \mu \mathrm{L}$ of antiGFP beads slurry (GFP-Trap ${ }^{\circledR}$ MA, \#gtma-10, Chemotek) or anti-HA bead slurry (Pierce ${ }^{\mathrm{TM}}$ Anti-HA Magnetic Beads, \#88836, Thermo Fisher Scientific) for GFP immunoprecipitation and HA immunoprecipitation, respectively, and incubated with gentle end-over-end mixing for $4 \mathrm{~h}$ at RT. Beads were washed three times with dilution buffer $(10 \mathrm{mM}$ Tris- $\mathrm{HCl} \mathrm{pH} 7.5$, $150 \mathrm{mM} \mathrm{NaCl}, 0.5 \mathrm{mM}$ EDTA pH 8.0) before boiling for $10 \mathrm{~min}$ at $70^{\circ} \mathrm{C}$. Protein complexes were resolved in 4-20\% Tris-glycine gels (BIO-RAD) using PageRuler ${ }^{\mathrm{TM}}$ Plus Prestained Protein Ladder (Thermo Fisher Scientific) as a marker and transferred onto nitrocellulose membranes (GE Healthcare Life Sciences). Membranes were probed with primary antibodies mouse anti-HA (\#MMS-101P, Covance, 1:1000) and mouse anti-GFP (\#600-301-215, Rockland, 1:1000) followed by incubation with secondary antibody goat anti-mouse IgGHRP (\#115-035-146, Jackson ImmunoResearch, 1:2000). Immobilon Forte Western HRP substrate (\#WBLUF0100, Millipore) was used for detection. Protein concentration was determined with Pierce ${ }^{\mathrm{TM}}$ BCA Protein Assay Kit (\#23227, Thermo Fisher Scientific). 


\section{Pull-down assays}

HEK293T cells were plated at a density of $1 \times 10^{6}$ cells per $10 \mathrm{~cm}$ dish and transfected $24 \mathrm{~h}$ postseeding with mCherry-P2A-mOtof-HA or mCherry-P2A-HA (control) using Lipofectamine ${ }^{\oplus}$ 3000 (\#L3000015, Thermo Fisher Scientific). Cells were harvested 72h post-transfection by washing three times in PBS and lysed in NP-40 lysis buffer supplemented with protease inhibitors by pipetting extensively for $1 \mathrm{~h}$ on ice and centrifuged at $500 \mathrm{x} \mathrm{g}, 4^{\circ} \mathrm{C}$ for $5 \mathrm{~min}$ to remove cell debris. Lysates were mixed with $25 \mu \mathrm{L}$ of anti-HA bead slurry (Pierce ${ }^{\mathrm{TM}}$ Anti-HA Magnetic Beads, \#88836, Thermo Fisher Scientific) incubated with gentle end-over-end mixing for $1 \mathrm{~h}$ at $4{ }^{\circ} \mathrm{C}$. Beads were washed three times with dilution buffer $(10 \mathrm{mM}$ Tris- $\mathrm{HCl}$ pH 7.5, $150 \mathrm{mM} \mathrm{NaCl}, 0.5 \mathrm{mM}$ EDTA pH 8.0).

OCs from 25 mice at P8-P9 were homogenized in ice-cold sucrose buffer (320 mM sucrose, 4 $\mathrm{mM}$ HEPES, pH 7.4, supplemented with protease inhibitors (\#4693132001, Roche, cOmplete $^{\mathrm{T} \mathrm{M}}$, EDTA-free Protease Inhibitor Cocktail)) using a glass-Teflon homogenizer, with 10 strokes at 900 r.p.m (adapted from Huttner et al, 1983; Hell \& Jahn, 2006; Ahmed et al, 2013). The homogenate was centrifuged at $500 \mathrm{x} \mathrm{g}, 4^{\circ} \mathrm{C}$ for $5 \mathrm{~min}$ to remove bone and cell debris. Homogenates (500 $\mu \mathrm{g}$ total protein) were loaded onto anti-HA beads previously immobilized with HA or otoferlin-HA proteins, and incubated with gentle end-over-end mixing overnight at $4{ }^{\circ} \mathrm{C}$. Beads were washed three times with dilution buffer prior to elution by boiling for $10 \mathrm{~min}$ at $70{ }^{\circ} \mathrm{C}$.

Protein complexes were resolved in 4-20\% Tris-glycine gels (BIO-RAD) using PageRuler ${ }^{\mathrm{TM}}$ Plus Prestained Protein Ladder (Thermo Fisher Scientific) as a marker and transferred onto nitrocellulose membranes (GE Healthcare Life Sciences). Membranes were probed with primary antibodies rabbit anti-PKC alpha (\#ab32376, Abcam, 1:1000), mouse anti-calbindin D-28K (\#CB300, Swant, 1:1000) and mouse anti-HA (\#MMS-101P, Covance, 1:1000) followed by incubation with secondary antibodies goat anti-rabbit IgG $(\mathrm{H}+\mathrm{L})-\mathrm{HRP}$ (\#111035-144, Jackson ImmunoResearch, 1:2000), goat anti-mouse Fc $\gamma$ fragment specific-HRP (\#115-035-008, Jackson ImmunoResearch, 1:2000), goat anti-mouse IgG (H+L)-HRP (\#115035-146, Jackson ImmunoResearch, 1:2000), respectively. Immobilon Forte Western HRP substrate (\#WBLUF0100, Millipore) was used for detection. Protein concentration was determined with Pierce ${ }^{\mathrm{\tau M}}$ BCA Protein Assay Kit (\#23227, Thermo Fisher Scientific).

\section{In vitro phosphorylation assay and mass spectrometry analysis}

HA-tagged otoferlin was overexpressed in HEK293T cells and immobilized onto anti-HA beads (Pierce ${ }^{\mathrm{TM}}$ Anti-HA Magnetic Beads, \#88836, Thermo Fisher Scientific) as already described. To obtain dephosphorylated otoferlin-HA, after extensive washing in dilution buffer (10 mM Tris- $\mathrm{HCl} \mathrm{pH} 7.5,150 \mathrm{mM} \mathrm{NaCl}, 0.5$ mM EDTA pH 8.0), beads were incubated with equimolar amounts of alkaline phosphatase (Calf Intestinal Alkaline Phosphatase, 
\#18009019, Thermo Fisher Scientific) in dephosphorylation buffer $(50 \mathrm{mM}$ Tris- $\mathrm{HCl} \mathrm{pH}$ 8.5, $0.1 \mathrm{mM}$ EDTA) for $30 \mathrm{~min}$ at $37^{\circ} \mathrm{C}$. The reaction was stopped by incubation with phosphatase inhibitors (PhosSTOP EASYpack Roche, \#4906845001, Thermo Fisher Scientific) for $15 \mathrm{~min}$ at $25^{\circ} \mathrm{C}$. After extensive washing in dilution buffer to remove any residual phosphatase, half the sample was set aside (dephosphorylated control sample) and the other half proceeded for incubation with PKC. The kinase assay was carried out with equimolar amounts of recombinant PKC $\alpha$ (Recombinant human PKC alpha protein, ab55672, Abcam) in kinase buffer (20 mM HEPES pH 7.5, 2mM DTT, $2 \mathrm{mM} \mathrm{CaCl}_{2}, 5 \mathrm{mM} \mathrm{MgCl}_{2}, 200 \mathrm{nM}$ phorbol 12-myristate 13-acetate, $260 \mu \mathrm{M}$ phosphatidylserine, $100 \mu \mathrm{M}$ ATP) for $30 \mathrm{~min}$ at 37 ${ }^{\circ} \mathrm{C}$. The reaction was terminated by adding $4 \mathrm{X}$ NuPAGE LDS Sample Buffer supplemented with $10 \%$ beta-mercaptoethanol to the samples and boiling at $95^{\circ} \mathrm{C}$ for $10 \mathrm{~min}$. Protein samples were loaded onto a 4-12\% NuPAGE Novex Bis-Tris Minigels (Invitrogen). Following detection by Coomassie staining, protein bands were cut out, diced and subjected to reduction with dithiothreitol, alkylation with iodoacetamide and finally overnight digestion with trypsin. Tryptic peptides were extracted from the gel, the solution dried in a Speedvac and kept at $20^{\circ} \mathrm{C}$ for further analysis (Atanassov \& Urlaub, 2013).

Protein digests were analyzed on a nanoflow chromatography system (Eksigent nanoLC425) hyphenated to a hybrid triple quadrupole-TOF mass spectrometer (TripleTOF 5600+) equipped with a Nanospray III ion source (Ionspray Voltage $2400 \mathrm{~V}$, Interface Heater Temperature $150^{\circ} \mathrm{C}$, Sheath Gas Setting 12) and controlled by Analyst TF 1.7.1 software build 1163 (all AB Sciex). In brief, peptides were dissolved in loading buffer ( $2 \%$ acetonitrile, $0.1 \%$ formic acid in water), enriched on a micro pillar array trapping column $(1 \mathrm{~cm}, \mu \mathrm{Pac}, 5 \mu \mathrm{m}$, PharmaFluidics $)$ and separated on an analytical micro pillar array column $(200 \mathrm{~cm}, \mu \mathrm{Pac}, 2.5$ $\mu \mathrm{m}$, PharmaFluidics) using a $60 \mathrm{~min}$ linear gradient of $5-40 \%$ acetonitrile $/ 0.1 \%$ formic acid (v:v) at $450 \mathrm{nl} \mathrm{min-1.}$

Qualitative LC-MS/MS analysis was performed using a Top20 data-dependent acquisition method with an MS survey scan of $m / z$ 350-1250 accumulated for $250 \mathrm{~ms}$ at a resolution of 30,000 full width at half maximum (FWHM). MS/MS scans of $\mathrm{m} / \mathrm{z} 180-1600$ were accumulated for $85 \mathrm{~ms}$ at a resolution of 17,500 FWHM and a precursor isolation width of 0.7 FWHM, resulting in a total cycle time of $2.0 \mathrm{~s}$. Precursors above a threshold MS intensity of 125 cps with charge states 2+, 3+, and 4+ were selected for MS/MS, the dynamic exclusion time was set to $45 \mathrm{~s}$. MS/MS activation was achieved by CID using nitrogen as a collision gas and the manufacturer's default rolling collision energy settings. Two technical replicates per sample were analyzed.

Protein identification was achieved using Mascot Software 2.6 (Matrixscience). LC-MS/MS runs were searched against the UniProtKB Mus musculus reference proteome (revision 122017, 60,769 entries). The search was performed with trypsin as enzyme and iodoacetamide as cysteine blocking agent. Up to two missed tryptic cleavages, methionine oxidation and $\mathrm{S} / \mathrm{T} / \mathrm{Y}$ phosphorylation as variable modifications were allowed for. Search tolerances were set 
to $20 \mathrm{ppm}$ for the precursor mass and $0.05 \mathrm{Da}$ for fragment masses, and ESI-QUAD-TOF specified as the instrument type. Extracted Ion Chromatograms (XICs) were generated in PeakView Software version 2.1 build 11041 (AB Sciex) using $0.05 \mathrm{~m} / \mathrm{z}$ extraction windows.

\section{Immunohistochemistry and Proximity ligation assay}

For general immunostainings (Figure EV1) and quantification of total protein levels (Figures 3.4A-B, 3.7D-E, EV3), the apical turn of OCs from P14-16 mice was freshly dissected in phosphate buffered saline (PBS), directly fixed with $4 \%$ formaldehyde (FA) in phosphate buffered saline (PBS) for $45 \mathrm{~min}$ at $4{ }^{\circ} \mathrm{C}$.

In otoferlin rescue experiments, cochleae of P23-P30 mice were directly fixed with 4\% FA in PBS for $45 \mathrm{~min}$ at $4{ }^{\circ} \mathrm{C}$ and decalcified in $0.12 \mathrm{M}$ EDTA ( $\mathrm{pH} \mathrm{8.0)}$ for 2-3 days before dissection of the OCs.

Chemical stimulation was performed essentially as described before (Kamin et al, 2014; Revelo et al, 2014). The apical turn of the OC from P14-16 mice was dissected in Hank's Balanced Salt Solution without calcium HBSS (HBSS without $\mathrm{Ca}^{2+}$; composed of $5.36 \mathrm{mM} \mathrm{KCl,} 141.7$ $\mathrm{mM} \mathrm{NaCl}, 1 \mathrm{mM} \mathrm{MgCl} 2,0.5 \mathrm{mM} \mathrm{MgSO}_{4}, 10 \mathrm{mM}$ HEPES, $3.4 \mathrm{mM}$ L-glutamine, and 6.9 $\mathrm{mM}$ D-glucose, $\mathrm{pH} 7.4$ ) and then subjected to one of the following experimental conditions: i) Resting, $1 \mathrm{~min}$ in $\mathrm{HBSS}$ without $\mathrm{Ca}^{2+}$; b) Stimulation, $1 \mathrm{~min}$ in $\mathrm{HBSS}$ high $\mathrm{K}^{+}(\mathrm{KCl}$ increased to $65.36 \mathrm{mM}, \mathrm{NaCl}$ reduced to $79.7 \mathrm{mM}$, and $2 \mathrm{mM} \mathrm{CaCl}$ ); c) Recovery, same as stimulated, followed by incubation for $5 \mathrm{~min}$ in $\mathrm{HBSS}$ with $\mathrm{Ca}^{2+}(\mathrm{NaCl}$ reduced to $139.7 \mathrm{mM}$ plus $2 \mathrm{mM} \mathrm{CaCl}_{2}$, with $5.36 \mathrm{mM} \mathrm{KCl}$ ). To pharmacologically activate PKC, OCs were dissected in HBSS without $\mathrm{Ca}^{2+}$ and incubated for 1, 5 and 15 min in HBSS with $\mathrm{Ca}^{2+}$ supplemented with $1 \mu \mathrm{M}$ PMA (phorbol 12-myristate 13-acetate; \#ab120297, Abcam). To inhibit PKC, OCs were incubated for 15 min in HBSS with $\mathrm{Ca}^{2+}$ supplemented with $10 \mu \mathrm{M}$ BIM I (Bisindolylmaleimide I, \#203290, Merck) prior to stimulation with HBSS high $\mathrm{K}^{+}+$ BIM I. To pharmacologically inhibit both PKC and CaMKII, OCs were incubated for $15 \mathrm{~min}$ in HBSS with $\mathrm{Ca}^{2+}$ supplemented with $10 \mu \mathrm{M}$ BIM I and $50 \mu \mathrm{M}$ CaMKII inhibitor KN-93 (\#Cay13319, Cayman Chemical) prior to stimulation with HBSS high $\mathrm{K}^{+}+\mathrm{BIM} \mathrm{I}+\mathrm{KN}-93$. All incubations were carried out at $37^{\circ} \mathrm{C}$ and all solutions were prewarmed at $37^{\circ} \mathrm{C}$. OCs were subsequently fixed with $4 \% \mathrm{FA}$ in PBS for $45 \mathrm{~min}$ at $4{ }^{\circ} \mathrm{C}$.

Immunostainings were performed as previously described (Strenzke et al, 2016). The following primary antibodies were used: mouse anti-otoferlin [13A9] (\#ab53233, Abcam, 1:300), rabbit anti-PKC alpha [Y124] (\#ab32376, Abcam, 1:300), rabbit anti-calbindin D28k (\#CB-38a, Swant, 1:300), goat anti-calbindin D28k [C-20] (\#sc-7691, Santa Cruz Biotechnology, 1:100), rabbit anti-Vglut3 (\#135 203, Synaptic Systems, 1:300), rabbit anti-myosin VI (KA15) (\#M5187, Sigma-Aldrich, 1:300), and goat IgG anti-CtBP2 [E-16] (\#sc-5967, Santa Cruz Biotechnology, 1:100) to label the synaptic ribbons. The following secondary antibodies were used: Alexa Fluor 488-conjugated goat anti-mouse IgG (\#A11001, Thermo Fisher Scientific, 
1:200), Alexa Fluor 594- and Alexa Fluor 568-conjugated donkey anti-mouse IgG (\#A21203, \#A10037, Thermo Fisher Scientific, 1:200), Alexa Fluor 568-conjugated goat anti-rabbit IgG (\#A11011, Thermo Fisher Scientific, 1:200), Alexa Fluor 488-conjugated donkey anti-rabbit IgG (\#A21206, Thermo Fisher Scientific, 1:200), DyLight 405-conjugated donkey anti-goat IgG (\#705-475-003, Jackson ImmunoResearch, 1:200), and MFP 488-conjugated donkey anti-goat IgG (\#MFP-A1055, MoBiTec, 1:200).

Proximity ligation assay (Duolink, Sigma-Aldrich) was performed essentially as described elsewhere (Meese et al, 2017). The Duolink ${ }^{\circledast}$ In Situ Detection Reagents Red set was used. The following antibody combinations were used: mouse anti-otoferlin [13A9] (\#ab53233, Abcam, 1:500) with rabbit anti-PKC alpha [Y124] (\#ab32376, Abcam, 1:500) or rabbit antiphosphoserine (\#9332, Abcam, 1:300) or rabbit anti-calbindin D28k (\#CB-38a, Swant, 1:500) or rabbit anti-Vglut3 (\#135 203, Synaptic Systems, 1:500) or rabbit anti-myosin VI (KA-15) (\#M5187, Sigma-Aldrich, 1:500); mouse anti-calbindin D28k (\#CB300, Swant, 1:500) with rabbit anti-PKC alpha [Y124] (\#ab32376, Abcam, 1:500). To visualize hair cells, primary antibody goat anti-calbindin D28K [C-20] (\#sc-7691, Santa Cruz Biotechnology, 1:100) was combined with secondary antibody MFP488 donkey anti-goat IgG (\#MFP-A1055, MoBiTec, 1:200), or primary antibody guinea pig anti-Vglut3 (\#135 204, Synaptic Systems, 1:500) was combined with secondary antibody DyLight 405-conjugated donkey anti-guinea pig IgG (\#706-475-148, Jackson ImmunoResearch, 1:100).

\section{Confocal microscopy and image analysis}

Confocal images were acquired using a laser scanning confocal microscope Leica TCS SP5 (Leica Microsystems GmbH, Wetzlar, Germany) with a 10X air objective (0.4 NA) and a 63x glycerol-immersion objective (1.3 NA) for low and high magnification images, respectively. Exceptionally, confocal images in Figure 3.2 were acquired with a laser scanning confocal microscope Zeiss LSM800 with Airyscan (Carl Zeiss AG, Oberkochen, Germany) with a 63X oil-immersion objective (1.4 NA). All images from the same series were acquired with the same voltage/offset/pinhole settings and laser power.

Maximum intensity projections of optical confocal sections and single-stack images were generated using Fiji (Schindelin et al, 2012, https://fiji.sc/) and assembled for display in Adobe Illustrator (Adobe Systems). Color-coded 2D images were constructed in Fiji as 16-bit grayscale images to which the given color look-up table was applied. Colocalization analysis was performed using the "Coloc2" Fiji's plugin with Costes' autothreshold method (Costes et al, 2004).

Protein expression levels, immunofluorescence and PLA signals were quantified from high magnification 3D IHC images $(0.6 \mu \mathrm{m}$ z-stack step size, $2 X$ digital zoom) in Imaris 7.6.5. Protein expression levels were quantified using a custom written Matlab (Mathworks) routine integrated into Imaris as previously described (Strenzke et al, 2016). PLA puncta were 
identified via "Spots" tool as objects with a signal above a minimum threshold. IHCs were identified by calbindin or Vglut3 fluorescence and the "Surface" tool was used to create a volume for each individual cell. Puncta per cell were obtained via the "Split Spots Into Surface Objects" Matlab XTension from Imaris, which creates a new subset of Spots that contains only the Spots that lie inside each Surface (i.e. each cell). Summed fluorescence intensities of puncta per cell were used to calculate the PLA puncta fluorescence intensity per cell and were normalized to the resting condition. For each experimental condition at least two independent experiments were performed. The same experimental settings were used for each series.

\section{Otoferlin rescue experiments}

The viral vectors used for the otoferlin rescue experiments in Figure 3.7E and Figure EV3B were designed, produced, purified, and injected through the round window membrane (RWM) into the left cochlea of P5-6 wild-type (B6 and CDB6F1) control and CD1B6F1 otoferlin knock-out mice as described in (Al-Moyed et al, 2019). The following virus titers were used for postnatal RWM injections: AAV2/6.eGFP $\left(1.44 \times 10^{10} \mathrm{vg} / \mu \mathrm{l}\right)$, otoferlin dual-AAV2/6-TS half-vectors $(1: 1)\left(1.2 \times 10^{10} \mathrm{vg} / \mu \mathrm{l}\right)$, and otoferlin dual-AAV2/6-Hyb halfvectors $(1: 1)\left(1.38 \times 10^{10} \mathrm{vg} / \mu \mathrm{l}\right)$. AAV2/6.eGFP was used as a control virus.

Otoferlin and calbindin protein expression levels were quantified in transduced IHCs (P2330) and normalized to protein levels in IHCs of non-injected B6 wild-type mice as in (AlMoyed et al, 2019). The otoferlin protein levels were previously reported in (Al-Moyed et al, 2019) and were only replotted in this study to better visualize the effect of otoferlin rescue on calbindin protein levels in otoferlin dual-AAV transduced IHCs.

\section{Statistical analysis}

Data averages from at least two independent trials are depicted as mean \pm standard error of the mean (s.e.m.). $\mathrm{P} \leq 0.05$ value was considered significant and is denoted in figures as follows: ns $\mathrm{P}>0.05 ;{ }^{*} \mathrm{P} \leq 0.05 ;{ }^{* *} \mathrm{P} \leq 0.01 ;{ }^{* *} \mathrm{P} \leq 0.001$. Statistical parameters, significance, and sample size ( $\mathrm{N}$, animal numbers; $\mathrm{n}$, cell numbers) are reported in the figure legends. All data fitting and statistical analysis was performed using GraphPad Prism 7.03 (GraphPad Software). The D'Agostino-Pearson omnibus and the Shapiro-Wilk tests were used to test for normality. The Mann-Whitney test was used to test for statistical significance between two unpaired nonnormally distributed groups. The Kruskal-Wallis test followed by the Dunn's multiple comparison test was used to test for statistical significance in non-parametric multiple comparisons. 


\section{Data availability}

Raw data produced in this study are available upon request.

The mass spectrometry proteomics data have been deposited to the ProteomeXchange Consortium (http://proteomecentral.proteomexchange.org) via the PRIDE partner repository (Perez-Riverol et al, 2019) with the dataset identifier PXD015338.

\subsubsection{Acknowledgments}

The authors would like to thank Nina-Katrin Dankenbrink-Werder and Lisa Neuenroth for excellent technical assistance. We thank Ulrich Mueller for providing the pachanga mouse line. This work was supported by the University Medical Center Göttingen, the Deutsche Forschungsgemeinschaft (DFG) through the Collaborative Research Center SFB 889 (project A4 to ER and HU), the Heisenberg Program (to ER), and the Göttingen Graduate Center for Neurosciences, Biophysics, and Molecular Biosciences (GGNB) through a stipend to APC (DFG Grant GSC 226/4).

\subsubsection{Author Contributions}

APC and ER conceived the study. APC designed and cloned DNA constructs, carried out CoIP, pull-down and in vitro phosphorylation assays, performed immunohistochemistry and proximity ligation assays, acquired and analyzed confocal microscopy images. HAM performed otoferlin rescue experiments, corresponding immunohistochemistry and confocal microscopy image acquisition and analysis. CL designed and evaluated mass spectrometry experiments. APC, HAM and CL analyzed data and prepared figures. APC and ER wrote the manuscript with input from all authors. All authors revised the manuscript. ER and HU acquired funding.

\subsubsection{Conflict of interest}

The authors declare that the research was conducted in the absence of any commercial or financial relationships that could be construed as a potential conflict of interest. 


\subsubsection{Appendix}

A

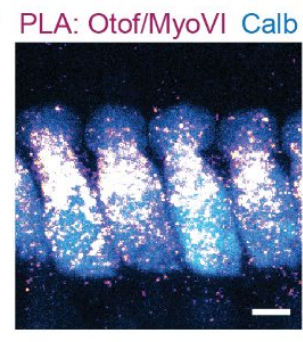

PLA: Otof/MyoVI

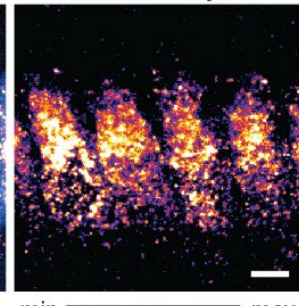

B

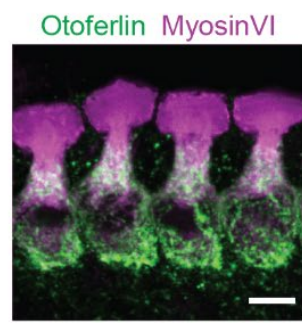

Otoferlin

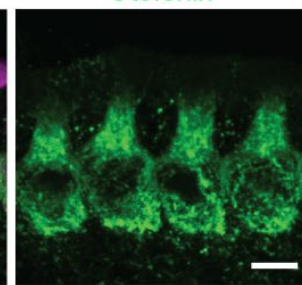

MyosinVI

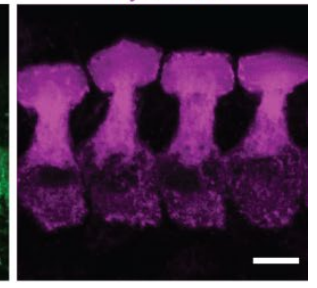

Appendix Figure S1. Validation of the proximity ligation assay in mice organs of Corti.

A High magnification views of a PLA assay for otoferlin and myosin VI performed on WT P14 IHCs. Calbindin (blue) was used as IHC marker. PLA channel is depicted with an intensity-coded lookup table with warmer colors representing higher pixel intensities.

B High magnification views of WT P14 IHCs immunolabeled for otoferlin and myosin VI with the antibodies used for the PLA shown in (A).

Data information: In (A-B), maximum intensity projections of confocal optical sections. Scale bars: $5 \mu \mathrm{m}$. PLA, proximity ligation assay. IHC, inner hair cell. Otof, otoferlin. MyoVI, myosin VI. Calb, calbindin. 


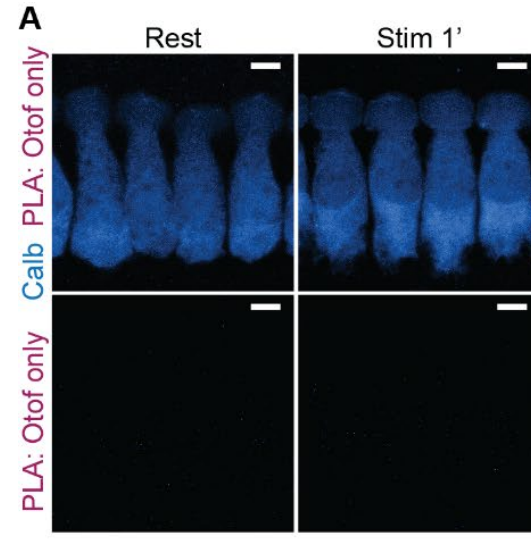

C

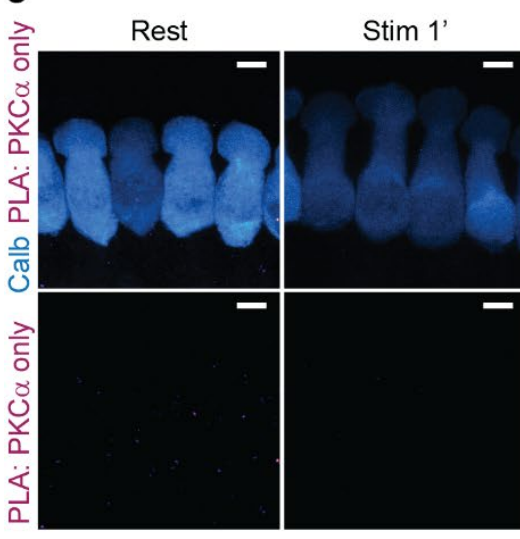

E

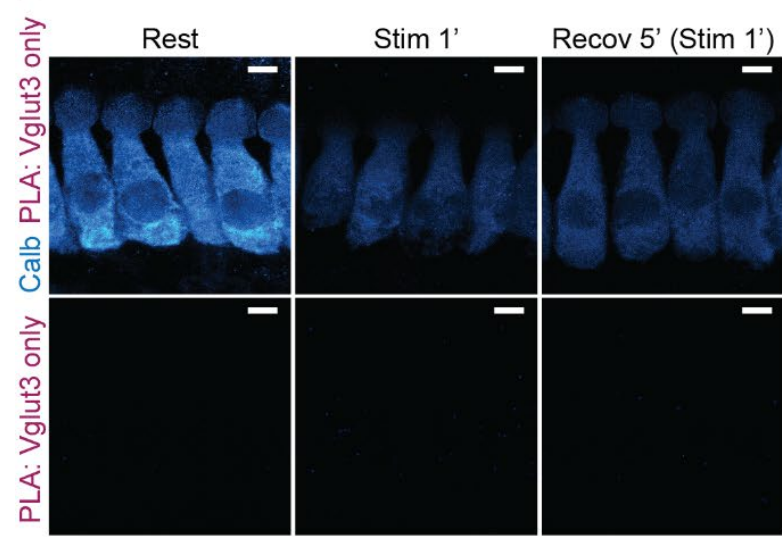

Stim 5

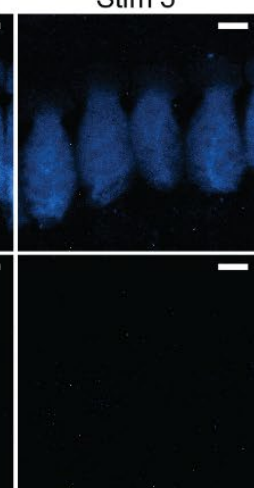

Recov 5' (Stim 1')
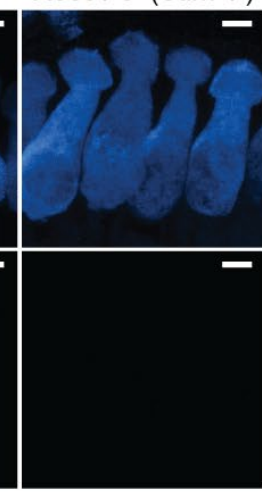

Recov 5' (Stim 1')

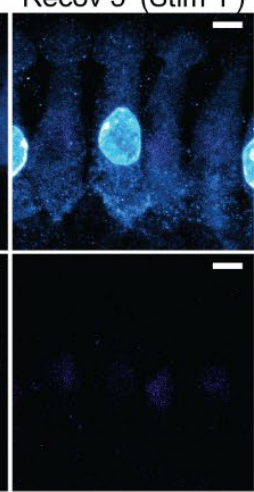

D

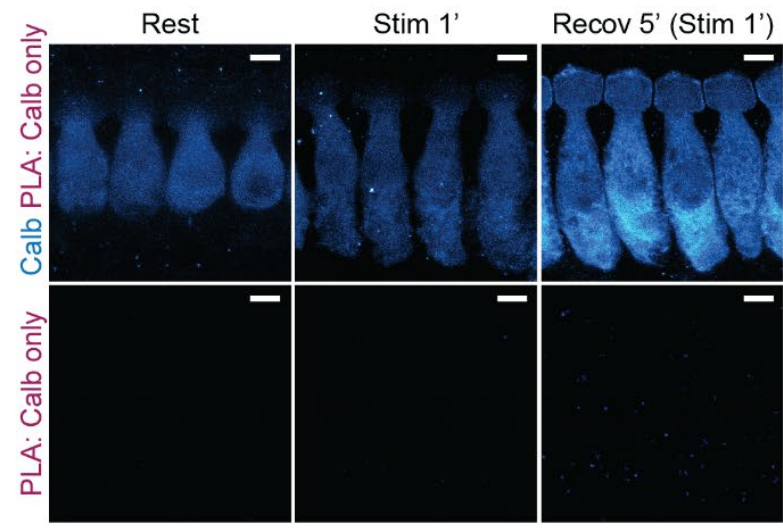

F

Stim 1

Recov 5' (Stim 1')
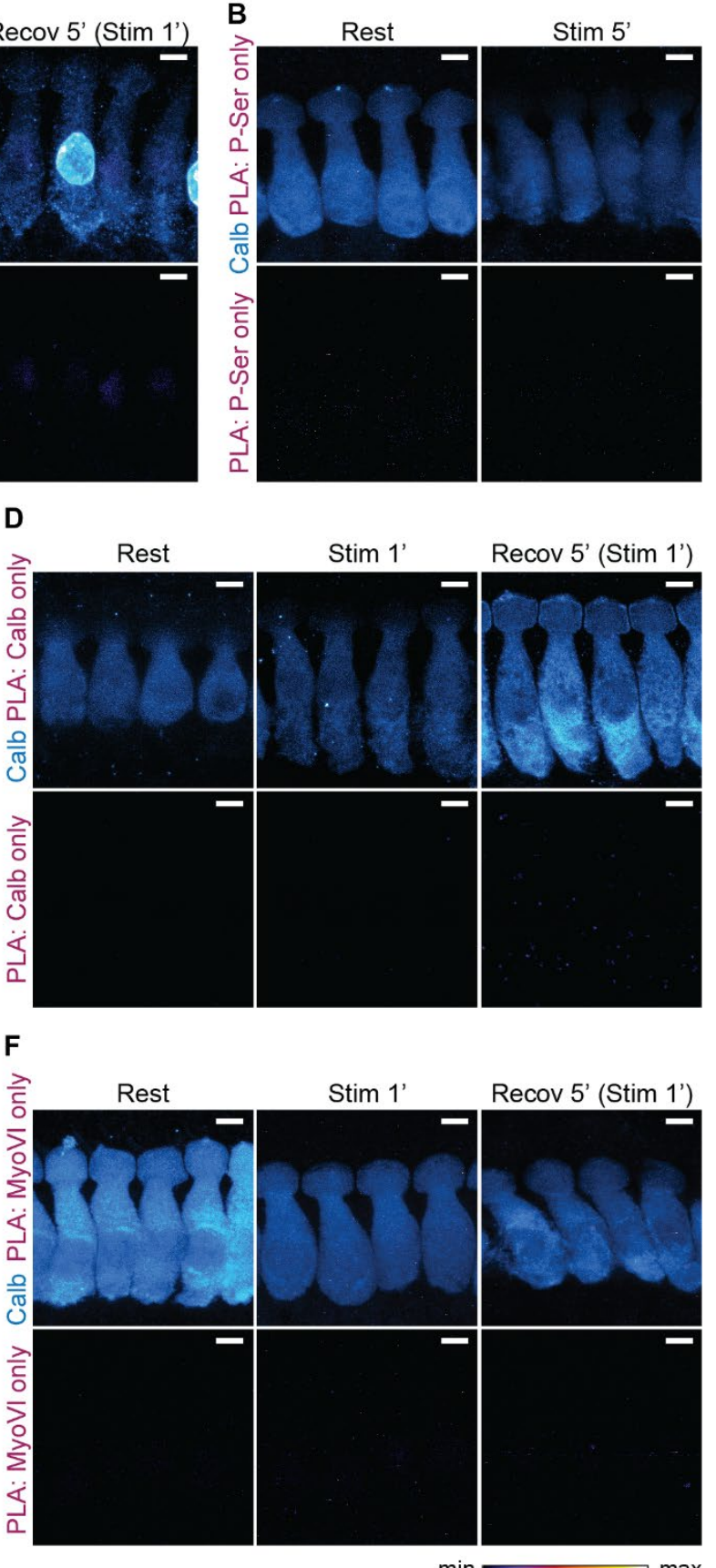

Appendix Figure S2. Negative controls for the proximity ligation assays.

A-F High magnification views of representative control PLAs performed with only one of the primary antibodies and done in parallel to the PLAs presented in this study: anti-otoferlin (A), antiphosphoserine (B), PKC $\alpha$ (C), calbindin (D), Vglut3 (E), myosin VI (F). Calbindin (blue) was used as IHC marker. The PLA channel is depicted with an intensity-coded lookup table (fire) with warmer colors representing higher pixel intensities. PLAs were performed for the conditions where the strongest PLA signal was registered in all different PLA combinations.

Data information: In (A-F), maximum intensity projections of confocal optical sections. Scale bars: $5 \mu \mathrm{m}$. Rest, resting; Stim 1', 1-minute stimulation; Stim 5', 5-minute stimulation; Recov 5' (Stim 1'), 5-minute recovery after 1-minute stimulation. PLA, proximity ligation assay. Calb, calbindin. Otof, otoferlin. P-Ser, phosphoserine. 
80 | Chapter 3

\section{Annotated MS/MS Spectra of Detected Phosphosites}

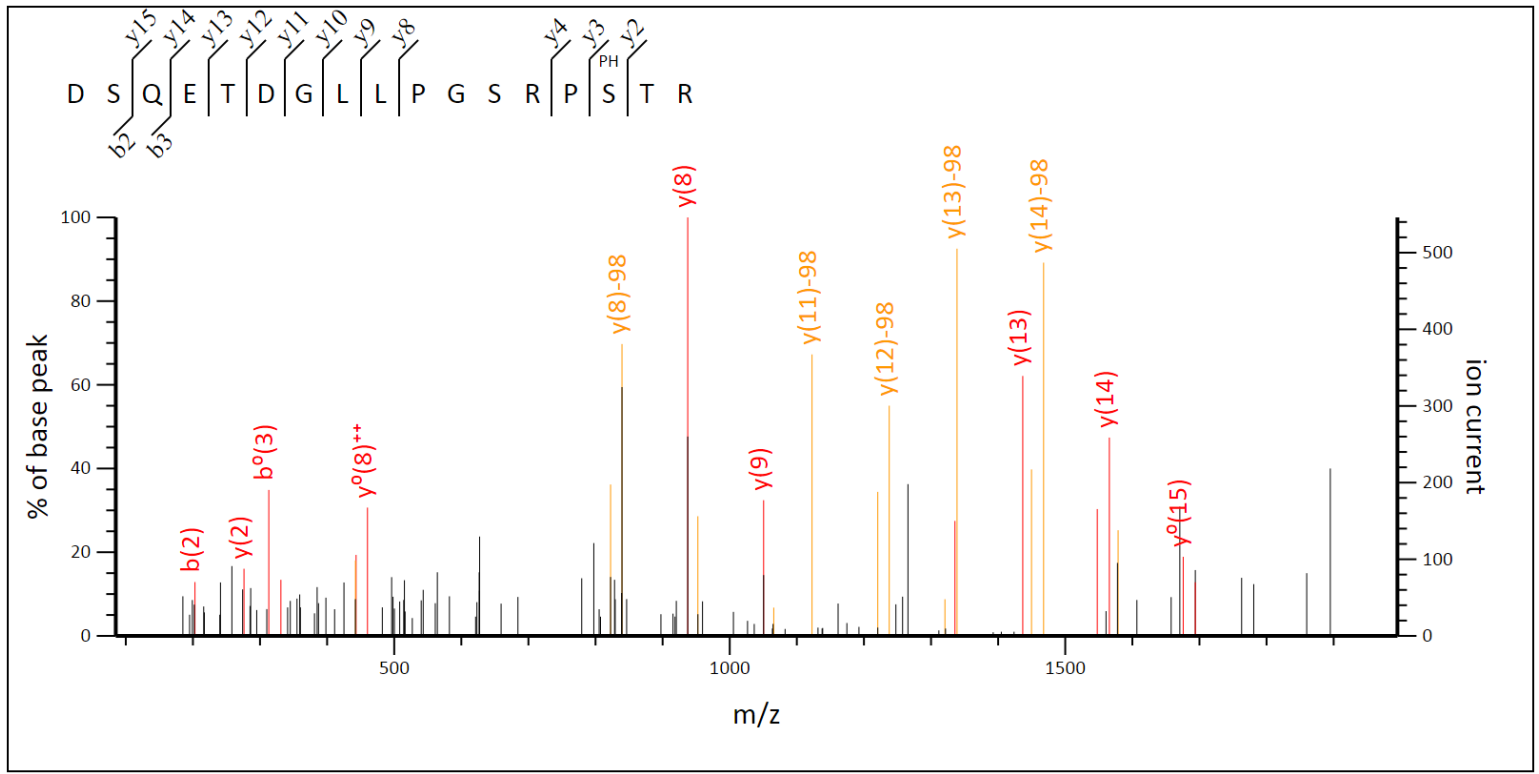

Appendix Figure S3. MS/MS spectrum of $\mathrm{m} / \mathrm{z} 632.629^{3+}$ at $38.63 \mathrm{~min}$, DSQETDGLLPGSRP ${ }^{158} \mathrm{pSTR}$ (otoferlin variant 1, NP_001093865.1).

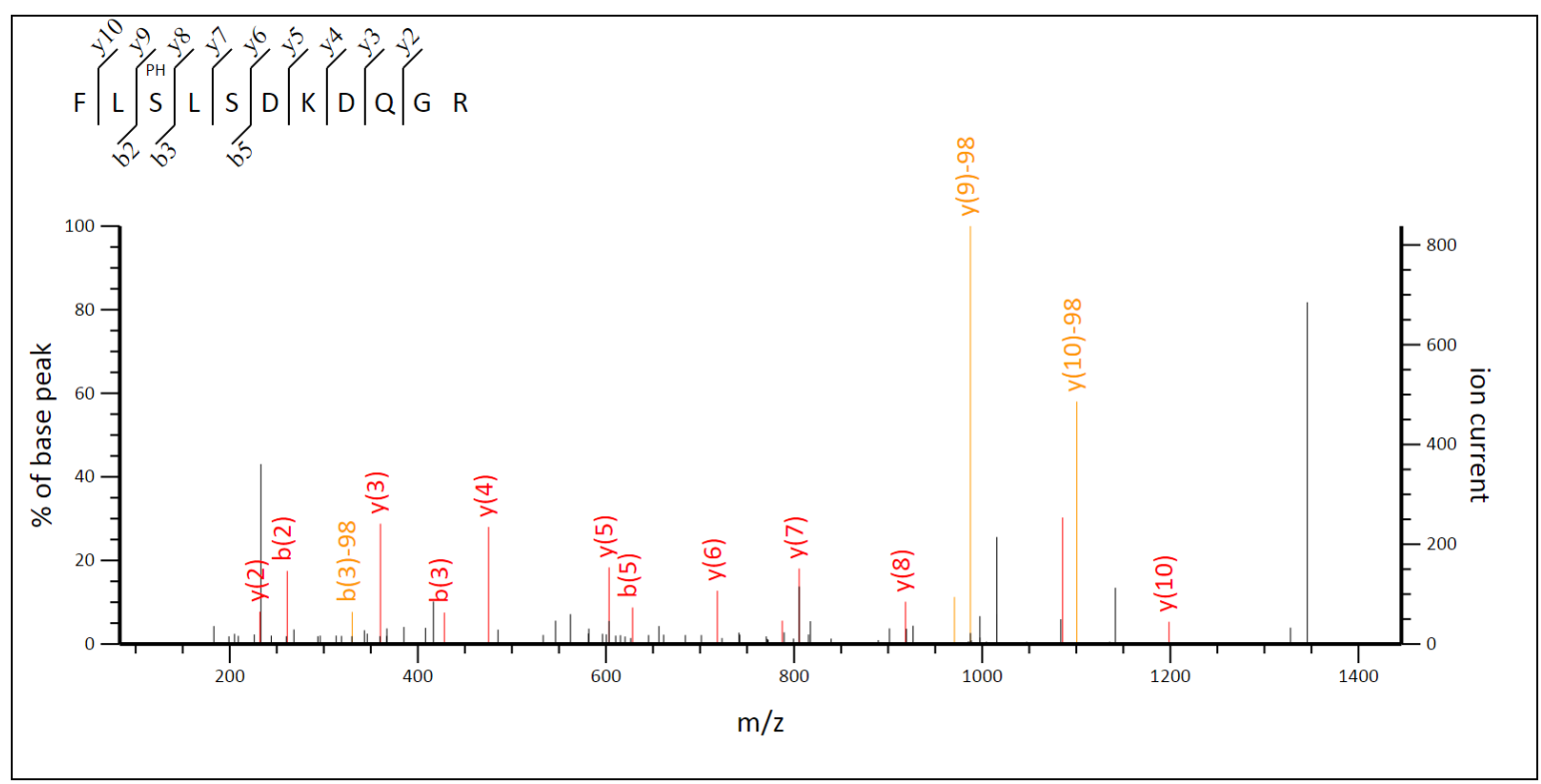

Appendix Figure S4. MS/MS spectrum of $\mathrm{m} / \mathrm{z} 449.209^{3+}$ at $42.93 \mathrm{~min}$, FL ${ }^{790} \mathrm{pSLSDKDQGR}$ (otoferlin variant 1, NP_001093865.1). 


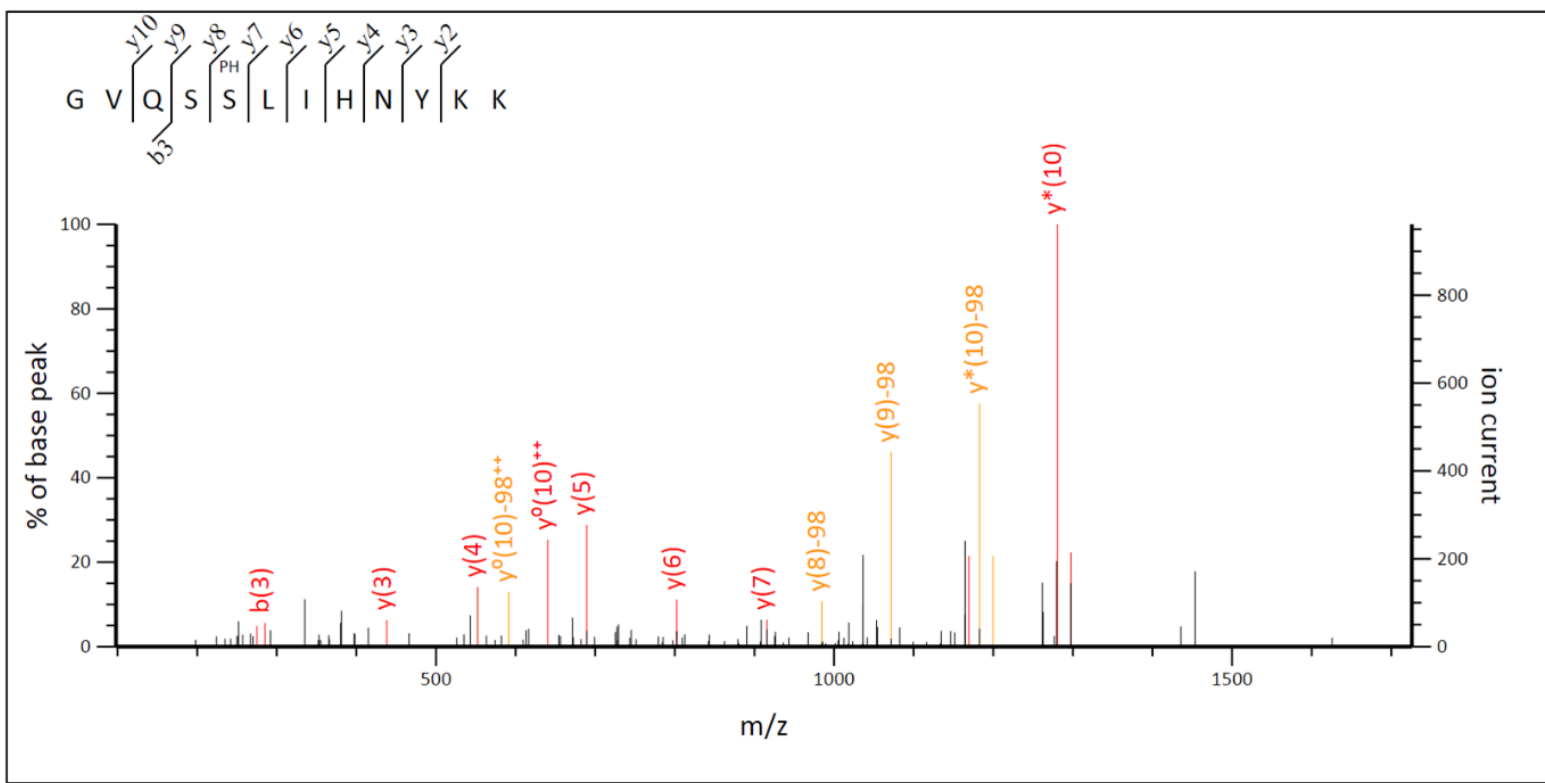

Appendix Figure S5. MS/MS spectrum of $\mathrm{m} / \mathrm{z} 485.245^{3+}$ at $32.04 \mathrm{~min}$, GVQS ${ }^{1184} \mathrm{pSLIHNYKK}$ (otoferlin variant 1, NP_001093865.1).

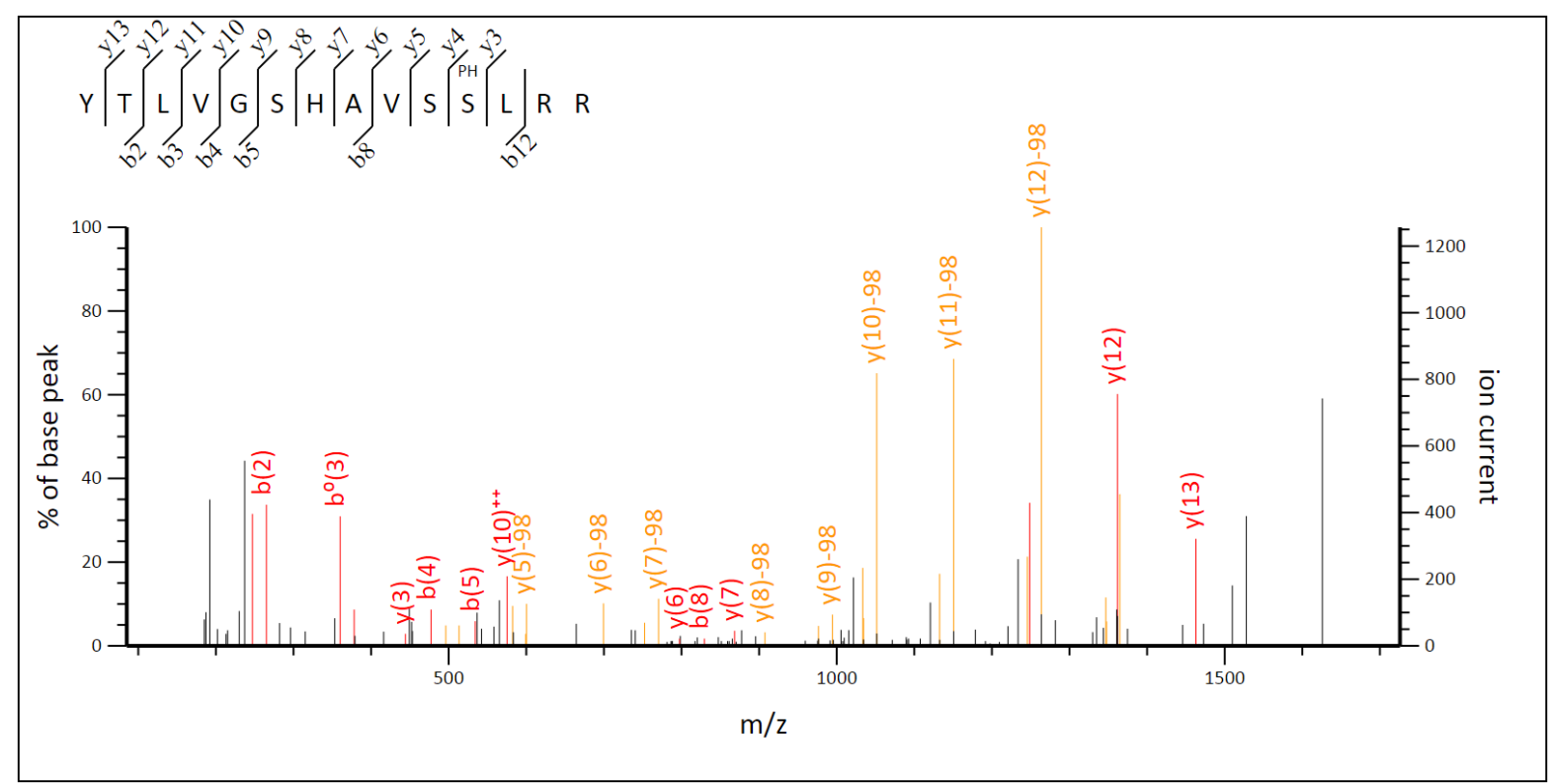

Appendix Figure S6. MS/MS spectrum of $m / z 542.614^{3+}$ at 38.08 min, YTLVGSHAVS ${ }^{1239}$ pSLRR (otoferlin variant 1, NP_001093865.1). 


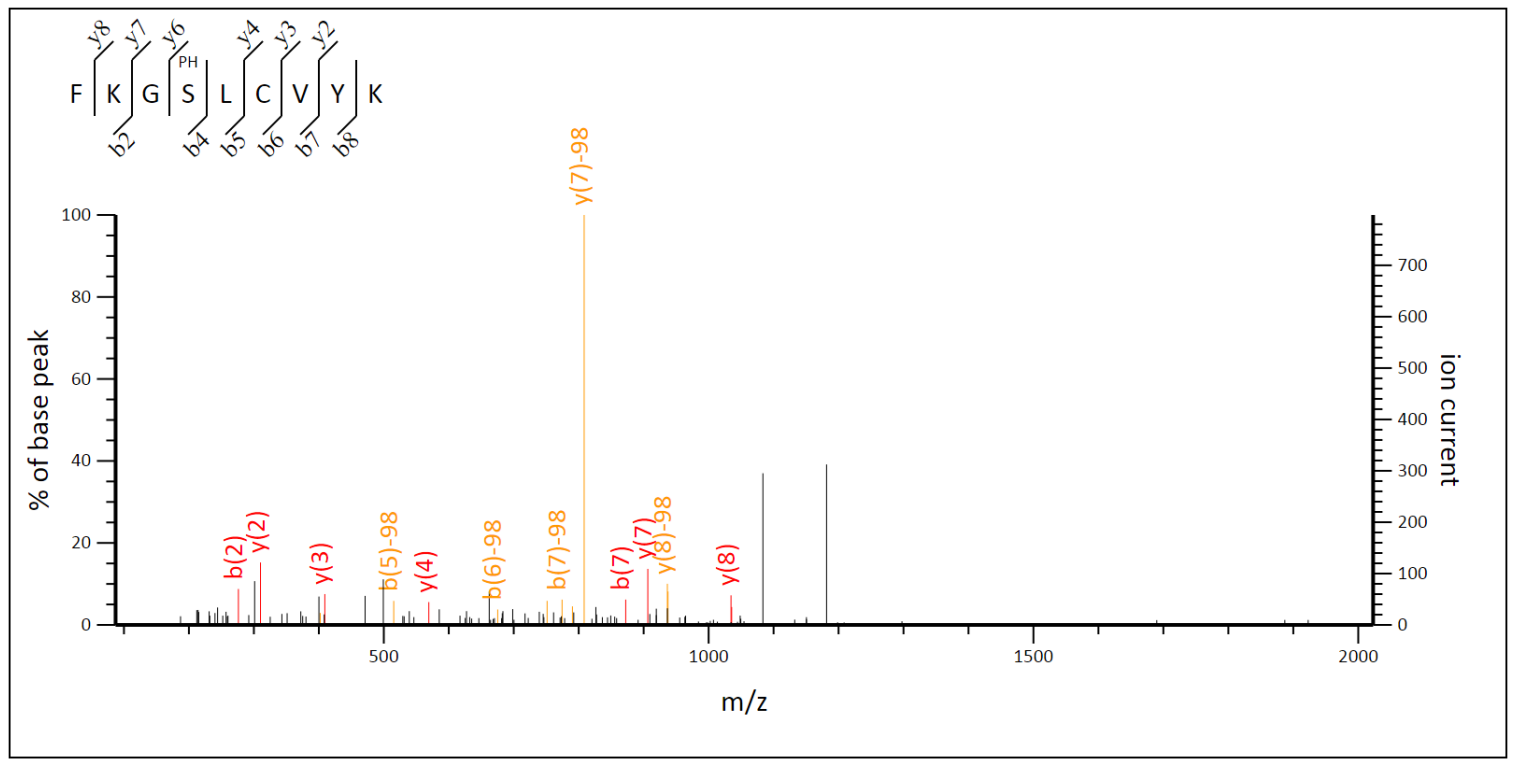

Appendix Figure S7. MS/MS spectrum of $\mathrm{m} / \mathrm{z} 591.280^{2+}$ at $37.87 \mathrm{~min}, \mathrm{FKG}^{1451} \mathrm{pSLCVYK}$ (otoferlin variant 1 , NP_001093865.1).

\section{LC-MS/MS profiling of phosphopeptides}

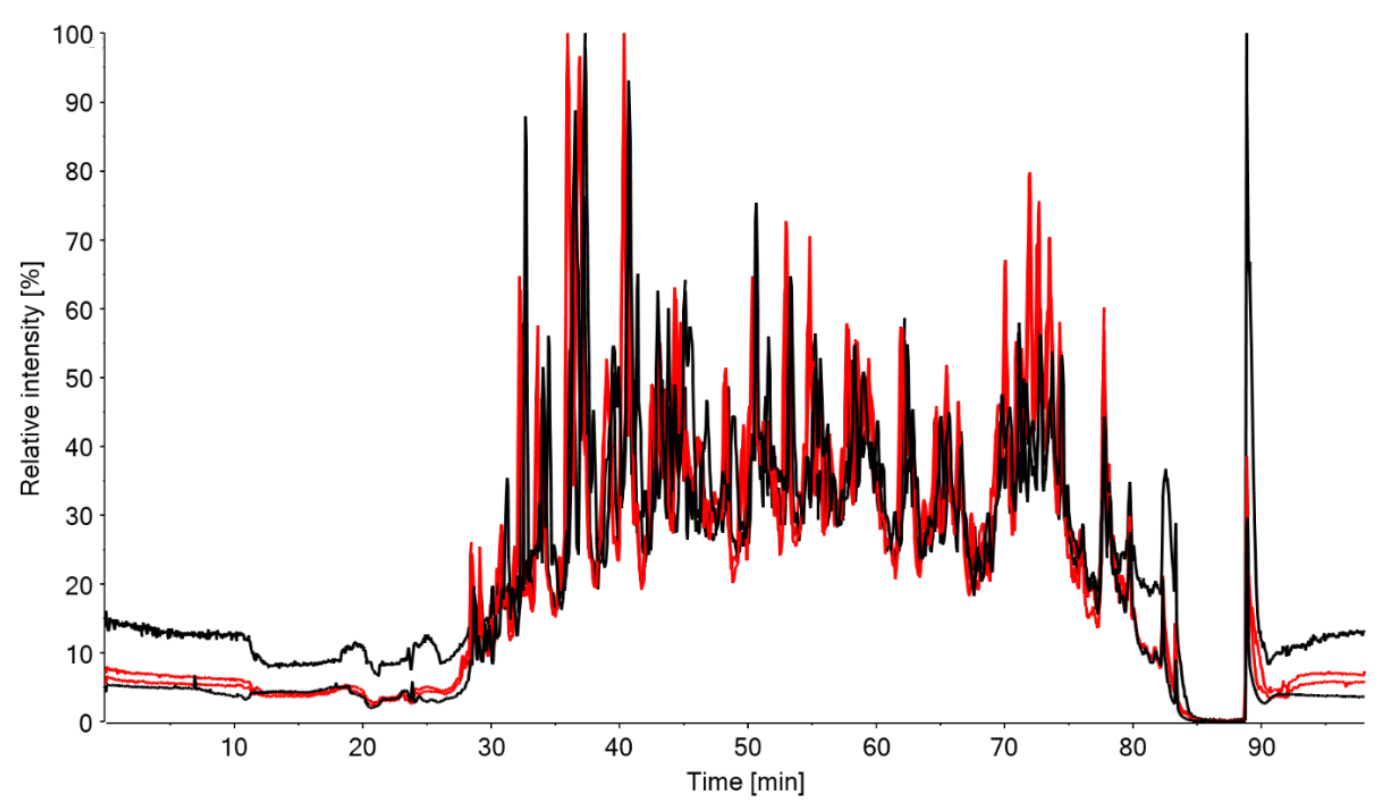

Appendix Figure S8. Total Ion Chromatograms (TICs) of otoferlin in-gel tryptic digests analyzed by LCMS/MS.

Two replicates each of phosphatase-treated (black) and phosphatase-treated/PKC-incubated (red) samples were analyzed. XIC overlays demonstrate excellent reproducibility. 

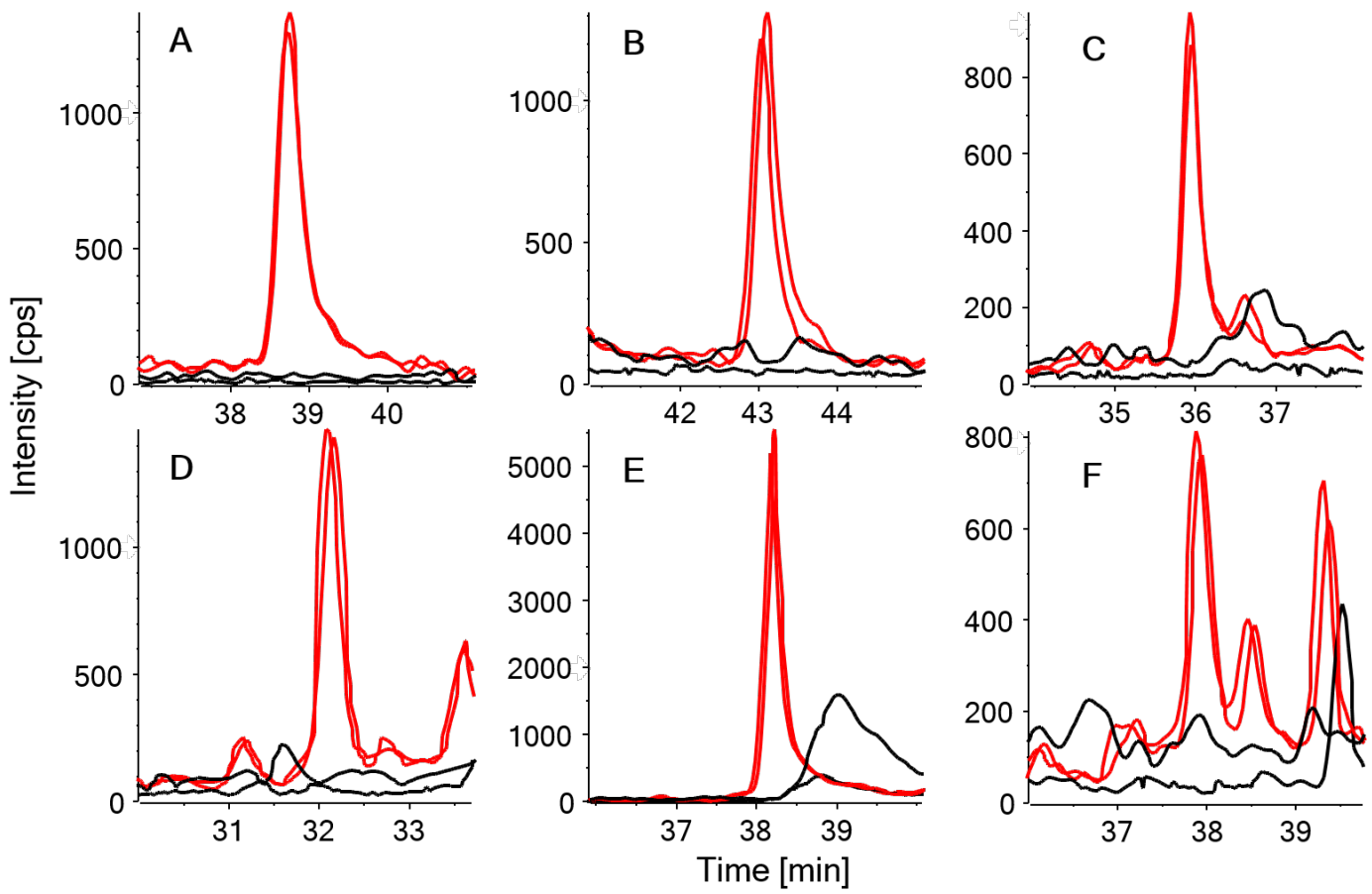

Appendix Figure S9. Extracted Ion Chromatograms (XICs) of otoferlin-derived phosphopeptides.

Two replicates each of phosphatase-treated (black) and phosphatase-treated/PKC-incubated (red) samples were analyzed.
A $\quad m / z 632.629^{3+}$ DSQETDGLLPGSRP $^{158} \mathrm{pSTR}$
B $\quad m / z 449.209^{3+} \mathrm{FL}^{790} \mathrm{pSLSDKDQGR}$
C $\quad m / z 663.322^{2+}$ GVQS $^{1184} \mathrm{pSLIHNYK}$
D $\quad m / z 485.245^{3+}$ GVQS $^{1184}$ PSLIHNYKK
E $\quad m / z 542.614^{3+}$ YTLVGSHAVS $^{1239}$ pSLRR
F $\quad m / z 591.280^{2+} \mathrm{FKG}^{1451} \mathrm{pSLCVYK}$

Background signal in the phosphatase-treated samples (black) indicates that phosphorylation was achieved by PKC incubation (red). 
motof ( $\operatorname{var} 1)$ motof (var4)

motof (varl) motof (var4)

motof ( $\operatorname{var} 1$ ) motof (var4)

motof (var1) motof (var4)

(...)

motof (var1) motof (var4)

(...)

motof (var1) motof (var4)

motof (var1) motof (var4)

(...)

motof (var1) motof (var4)

(...)

motof (var1) motof (var4)

motof (varl) motof (var 4$)$

\section{$\mathrm{C}_{2} \mathrm{~A}$}

MALIVHLKTVSELRGKGDRIAKVTFRGQSFYSRVLENCEGVADFDETFRWPVASSIDRNE 60 MALIVHLKTVSELRGKGDRIAKVT FRGQSFYSRVLENCEGVADFDETFRWPVASSIDRNE 60

\section{$\mathrm{C}_{2} \mathrm{~A}$}

VLEIQIFNYSKVFSNKLIGTFCMVLQKVVEENRVEVTDTLMDDSNAIIKTSLSMEVRYQA 120 VLEIQIFNYSKVFSNKL IGTFCMVLQKVVEENRVEVTDTLMDDSNAIIKTSLSMEVRYQA 120

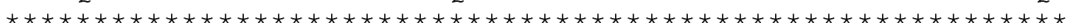

TDGTVGPWDDGDFLGDESLQEEKDSQETDGLLPGSRPSTRISGEKSFRSKGREKTKGGRD 180 TDGTVGPWDDGDFLGDESLQEEKDSQETDGLLPGSRPSTRISGEKSERR--------- 169

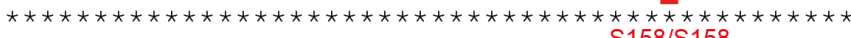
S158/S158

GEHKAGRSVFSAMKLGKTRSHKEE PQRQDEPAVLEMEDLDHLAIQLGDGLDPDSVSLASV 240 ----AGRSVFSAMKLGKTRSHKEE PQRQDEPAVLEMEDLDHLAIQLGDGLDPDSVSLASV 225

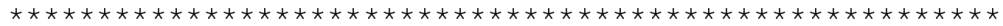

\section{FerA}

LSCGCHRFLSLSDKDQGRSSRTRLDRERLKSCMRELESMGQQAKSLRAQVKRHTVRDKLR 840 LSCGCHRFLSLSDKDQGRSSRTRLDRERLKSCMRELESMGQQAKSLRAQVKRHTVRDKLR 825

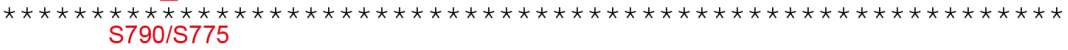

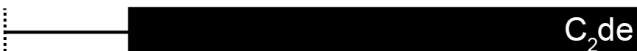

RPVLSKYRVEVLFWGLRDLKRVNLAQVDRPRVDIECAGKGVQSSLIHNYKKNPNFNTLVK 1200 RPVLSKYRVEVLFWGLRDLKRVNLAQVDRPRVDIECAGKGVQSSLIHNYKKNPNFNTLVK 1185

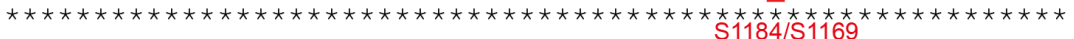

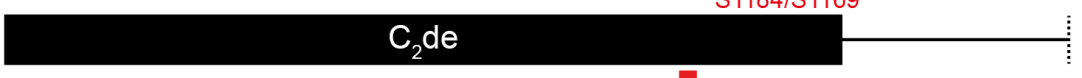

WFEVDLPENELLHPPLNIRVVDCRAFGRYTLVGSHAVSSLRRFIYRPPDRSAPNWNTTGE 1260 WFEVDLPENELLHP PLNIRVVDCRAFGRYTLVGSHAVSSLRRFIYRPPDRSAPNWNTTGE 1245

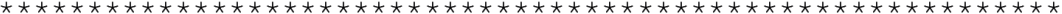
S1239/S1224

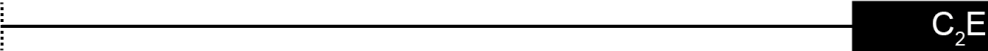

EERIVGRFKGSLCVYKVPLPEDVSREAGYDPTYGMFQGIPSNDPINVLVRIYVVRATDLH 1500 EERIVGRFKGSLCVYKVPLPEDVSREAGYDPTYGMFQGIPSNDPINVLVRIYVVRATDLH 1485

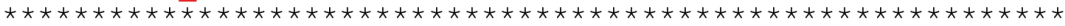
S1451/S1436

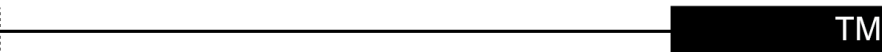

LARNEPDPLEKPNRPDTAFVWFLNPLKS IKYLICTRYKWLI IKIVLALLGLLMLALFLYS 1980 LARNEPDPLEKPNRPDTAFVWFLNPLKS IKYLICTRYKWLI IKIVLALLGLLMLALFLYS 1965

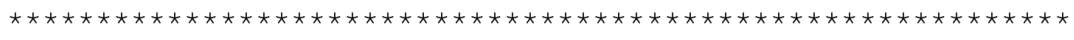

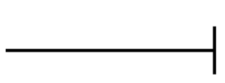

LPGYMVKKLLGA

1992

LPGYMVKKLLGA

1977

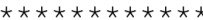

Appendix Figure S10. Sequence alignment of phosphorylated sites in otoferlin variants 1 (NP_001093865.1) and 4 (NP_001300696.1).

Phosphosites (red) identified at positions S158, S790, S1184, S1239, S1451 in variant 1 correspond to S158, S775, S1169, S1224, S1436 in variant 4, respectively. Alignment was performed using CLUSTAL Omega (1.2.4), EMBL-EBI. 


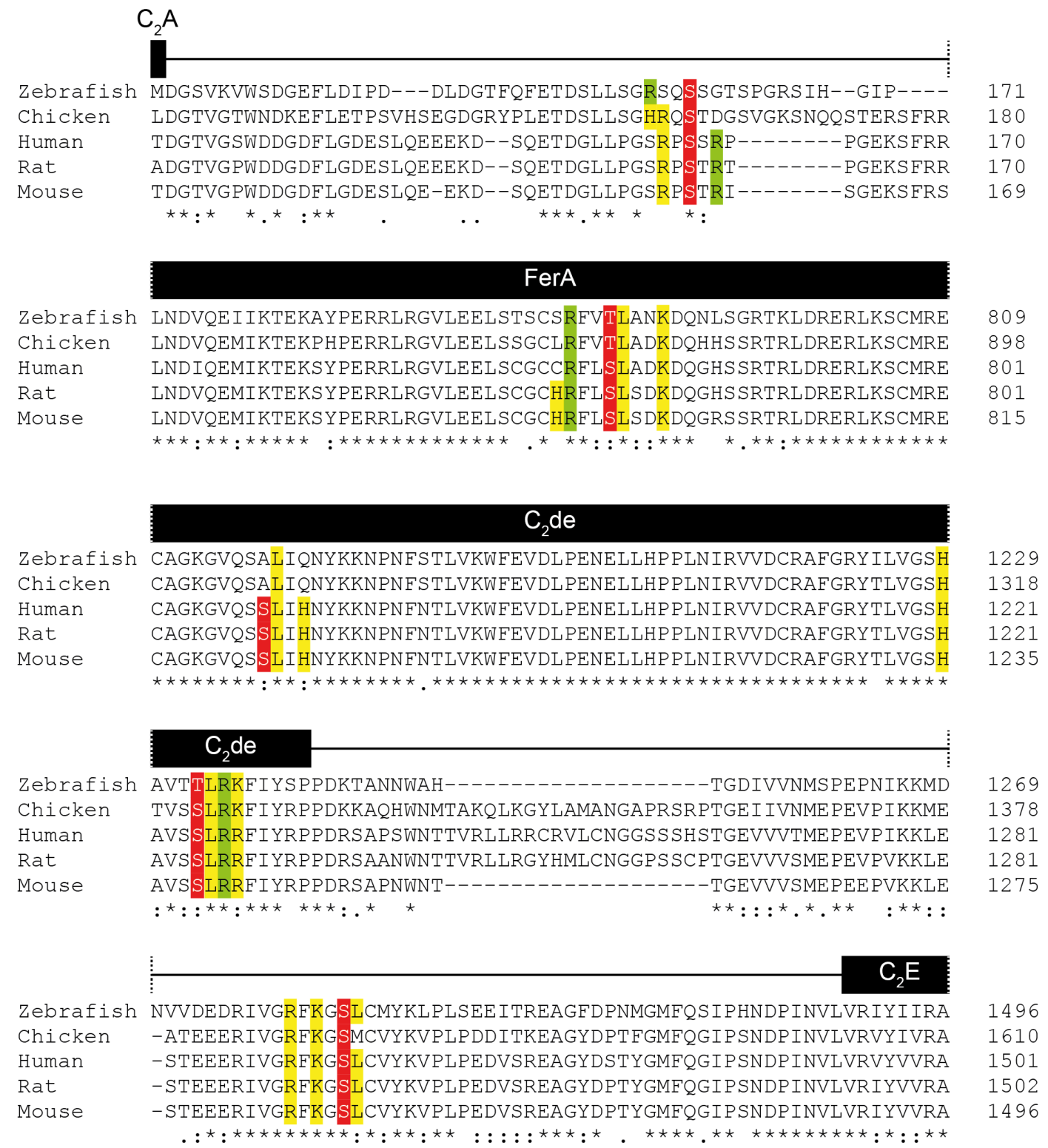

Appendix Figure S11. Sequence alignment of phosphorylated sites in otoferlin from different species. Identified phosphorylation sites in mouse otoferlin (NP_001093865.1, variant 1) were aligned with human (NP_919224.1), rat (NP_001263649.1), chicken (XP_015140684.1) and zebrafish (NP_001025283.1) otoferlin sequences (displayed in red). PKC consensus motif (R-X-X-S/T-X-R-X) with with respective arginines $(\mathrm{R})$ indicated in green; $\mathrm{X}$ indicates any amino acid. Hydrophobic leucine residues $(\mathrm{L})$ at the +1 position shown to be favored by PKC and reported variations to the consensus motif with basic amino acids (R, H, K) at positions $-6,-4,-2,+2,+3$, and +4 (Nishikawa et al, 1997) are depicted in yellow. Alignment was performed using CLUSTAL Omega (1.2.4), EMBL-EBI. 
86 | Chapter 3

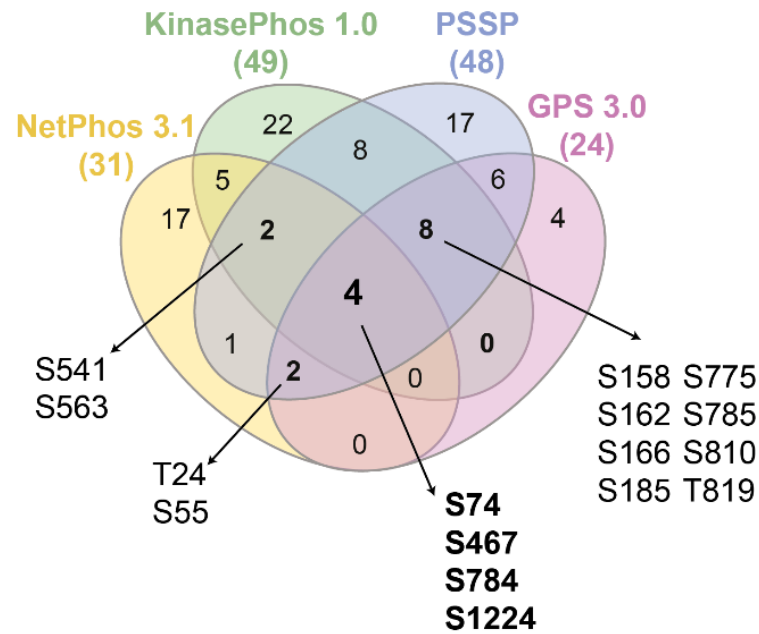

Appendix Figure S12. PKC is predicted to phosphorylate otoferlin.

Analysis of putative PKC phosphorylation sites in otoferlin (mouse, isoform 4, NP_001300696.1) using four different prediction tools (see Appendix Table S2 for detailed analysis). A comparative analysis between tools is represented as Veen Diagram. Numbers in parenthesis refer to total number of sites for each tool. Common sites to all tools are displayed in bold. Common sites found in at least three of the tools are also depicted. 
Appendix Table S1. Mean averages, sample size and statistical analysis.

Data information: s.e.m., standard error of the mean; N, number of animals; $n$, number of cells.

Figure 3.1D

\begin{tabular}{|l|c|c|c|c|}
\hline \multirow{2}{*}{ Genotype/Condition } & \multicolumn{2}{|c|}{ Mean \pm s.e.m. } & \multirow{2}{*}{$\mathbf{N}$} \\
\cline { 2 - 3 } & Otoferlin & PKC $\alpha$ & & \\
\hline Rest & $1.03 \pm 0.02$ & $1.05 \pm 0.03$ & 6 & 150 \\
\hline Stim 1' & $0.89 \pm 0.01$ & $0.76 \pm 0.02$ & 7 & 179 \\
\hline Stim 5' & $0.94 \pm 0.06$ & $0.85 \pm 0.06$ & 1 & 37 \\
\hline Recov 5' (Stim 1') & $1.60 \pm 0.04$ & $1.32 \pm 0.04$ & 6 & 195 \\
\hline
\end{tabular}

\begin{tabular}{|c|c|c|c|}
\hline Compared group & $\begin{array}{l}\text { Statistical } \\
\text { significance }\end{array}$ & $P$-value & Statistical test \\
\hline \multicolumn{4}{|l|}{ Apical/basal Otoferlin ratio: } \\
\hline Rest vs. Stim 1' & *** & 0.0004 & \multirow{6}{*}{$\begin{array}{l}\text { Kruskal-Wallis test followed by } \\
\text { Dunn's multiple comparison test }\end{array}$} \\
\hline Rest vs. Stim 5' & ns & 0.0658 & \\
\hline Rest vs. Recov 5' (Stim 1') & $* * *$ & $<0.0001$ & \\
\hline Stim 1' vs. Stim 5' & $\mathrm{ns}$ & $>0.9999$ & \\
\hline Stim 1' vs. Recov 5' (Stim 1') & $* * *$ & $<0.0001$ & \\
\hline Stim 5' vs. Recov 5' (Stim 1') & $* * *$ & $<0.0001$ & \\
\hline \multicolumn{4}{|l|}{ Apical/basal PKC $\alpha$ ratio: } \\
\hline Rest vs. Stim 1' & $* * *$ & $<0.0001$ & \multirow{6}{*}{$\begin{array}{l}\text { Kruskal-Wallis test followed by } \\
\text { Dunn's multiple comparison test }\end{array}$} \\
\hline Rest vs. Stim 5' & ** & 0.0080 & \\
\hline Rest vs. Recov 5' (Stim 1') & $* * *$ & $<0.0001$ & \\
\hline Stim 1' vs. Stim 5' & ns & $>0.9999$ & \\
\hline Stim 1' vs. Recov 5' (Stim 1') & $* * *$ & $<0.0001$ & \\
\hline Stim 5' vs. Recov 5' (Stim 1') & $* * *$ & $<0.0001$ & \\
\hline
\end{tabular}

Figure $3.3 \mathrm{~B}$

\begin{tabular}{|l|c|c|c|}
\hline \multicolumn{1}{|c|}{ Genotype/Condition } & Mean \pm s.e.m. & N & n \\
\hline Rest & $100 \pm 7 \%$ & 7 & 122 \\
\hline Stim 1' & $442 \pm 28 \%$ & 8 & 141 \\
\hline Recov 5' (Stim 1') & $178 \pm 7 \%$ & 6 & 112 \\
\hline
\end{tabular}

\begin{tabular}{|c|c|c|c|}
\hline Compared group & $\begin{array}{l}\text { Statistical } \\
\text { significance }\end{array}$ & $P$-value & Statistical test \\
\hline \multicolumn{4}{|l|}{ PLA Otoferlin/PKC $\alpha$ : } \\
\hline Rest vs. Stim 1' & $* * *$ & $<0.0001$ & \multirow{3}{*}{$\begin{array}{l}\text { Kruskal-Wallis test followed by } \\
\text { Dunn's multiple comparison test }\end{array}$} \\
\hline Rest vs. Recov 5' (Stim 1') & $* * *$ & $<0.0001$ & \\
\hline Stim 1' vs. Recov 5' (Stim 1') & $* * *$ & $<0.0001$ & \\
\hline
\end{tabular}

Figure 3.4B-C

\begin{tabular}{|l|c|c|c|c|}
\hline \hline \multirow{2}{*}{ Genotype/Condition } & \multicolumn{2}{|c|}{ Mean \pm s.e.m. } & N & \\
\cline { 2 - 3 } & Immunofluorescence & Apical/basal ratio & & \\
\hline WT & $100 \pm 2 \%$ & $1.06 \pm 0.03$ & 6 & 233 \\
\hline Otof $^{-/}$ & $92 \pm 1 \%$ & $0.66 \pm 0.02$ & 6 & 205 \\
\hline
\end{tabular}




\begin{tabular}{|l|c|c|c|}
\hline \multicolumn{1}{|c|}{ Compared group } & $\begin{array}{c}\text { Statistical } \\
\text { significance }\end{array}$ & $P$-value & Statistical test \\
\hline PKCa immunofluorescence: & ${ }^{* *}$ & 0.0054 & Mann-Whitney two-tailed t-test \\
\hline WT vs. Otof ${ }^{--}$ & & \\
\hline Apical/basal PKC ratio: & ${ }^{* * *}$ & $<0.0001$ & Mann-Whitney two-tailed t-test \\
\hline WT vs. Otof ${ }^{-/}$
\end{tabular}

Figure 3.4G

\begin{tabular}{|l|c|c|c|}
\hline \multicolumn{1}{|c|}{ Genotype/Condition } & Mean \pm s.e.m. & $\mathbf{N}$ & $\mathbf{n}$ \\
\hline WT Rest & $1.05 \pm 0.03$ & 6 & 150 \\
\hline WT Stim 1' & $0.76 \pm 0.02$ & 7 & 179 \\
\hline WT Stim 5' & $0.85 \pm 0.06$ & 1 & 37 \\
\hline WT Recov 5' (Stim 1') $^{\prime}$ Otof $^{-/}$Rest & $1.32 \pm 0.04$ & 6 & 195 \\
\hline Otof $^{-/}$Stim 1' & $1.15 \pm 0.12$ & 1 & 15 \\
\hline Otof $^{-/}$Stim 5' & $0.75 \pm 0.03$ & 2 & 50 \\
\hline Otof $^{-/}$Recov 5' (Stim 1') & $0.81 \pm 0.03$ & 2 & 46 \\
\hline
\end{tabular}

\begin{tabular}{|c|c|c|c|}
\hline Compared group & $\begin{array}{c}\text { Statistical } \\
\text { significance }\end{array}$ & $P$-value & Statistical test \\
\hline \multicolumn{4}{|l|}{ WT vs. Otof ${ }^{-/}$apical/basal PKCa ratio: } \\
\hline WT Rest vs. Otof ${ }^{-/}$Rest & ns & $>0.9999$ & \multirow{28}{*}{$\begin{array}{c}\text { Kruskal-Wallis test } \\
\text { followed by Dunn's } \\
\text { multiple } \\
\text { comparison test }\end{array}$} \\
\hline WT Rest vs. WT Stim 1' & $* * *$ & $<0.0001$ & \\
\hline WT Rest vs. Otof ${ }^{-/-}$Stim 1' & $* * *$ & $<0.0001$ & \\
\hline WT Rest vs. WT Stim 5' & $*$ & 0.0101 & \\
\hline WT Rest vs. Otof ${ }^{--}$Stim $5^{\prime}$ & $* *$ & 0.0051 & \\
\hline WT Rest vs. WT Recov 5' (Stim 1') & $* * *$ & $<0.0001$ & \\
\hline WT Rest vs. Otof ${ }^{--}$Recov 5' (Stim 1') & ns & 0.1708 & \\
\hline Otof ${ }^{-/-}$Rest vs. WT Stim 1' & $* *$ & 0.0011 & \\
\hline Otof $^{-/-}$Rest vs. Otof ${ }^{--}$Stim 1' & ** & 0.0023 & \\
\hline Otof ${ }^{-/}$Rest vs. WT Stim 5' & ns & 0.0754 & \\
\hline Otof ${ }^{--}$Rest vs. Otof ${ }^{--}$Stim $5^{\prime}$ & ns & 0.0740 & \\
\hline Otof ${ }^{-/-}$Rest vs. WT Recov 5' (Stim 1') & ns & $>0.9999$ & \\
\hline Otof ${ }^{-/}$Rest vs. Otof ${ }^{-/}$Recov 5' (Stim 1') & ns & 0.2537 & \\
\hline WT Stim 1' vs. Otof $f^{-/}$Stim 1' & $\mathrm{ns}$ & $>0.9999$ & \\
\hline WT Stim 1' vs. WT Stim 5' & $\mathrm{ns}$ & $>0.9999$ & \\
\hline WT Stim 1' vs. Otof ${ }^{-1-}$ Stim 5' & ns & $>0.9999$ & \\
\hline WT Stim 1' vs. WT Recov 5' (Stim 1') & *** & $<0.0001$ & \\
\hline WT Stim 1' vs. Otof ${ }^{-/}$Recov 5' (Stim 1') & ns & $>0.9999$ & \\
\hline Otof ${ }^{--}$Stim 1' vs. WT Stim 5' & ns & $>0.9999$ & \\
\hline Otof ${ }^{-/}$Stim 1' vs. Otof ${ }^{-/-}$Stim $5^{\prime}$ & ns & $>0.9999$ & \\
\hline Otof ${ }^{-/}$Stim 1' vs. WT Recov 5' (Stim 1') & $* * *$ & $<0.0001$ & \\
\hline Otof ${ }^{-/}$Stim 1' vs. Otof ${ }^{--}$Recov 5' (Stim 1') & ns & $>0.9999$ & \\
\hline WT Stim 5' vs. Otof ${ }^{-<}$Stim $5^{\prime}$ & ns & $>0.9999$ & \\
\hline WT Stim 5' vs. WT Recov 5' (Stim 1') & $* * *$ & $<0.0001$ & \\
\hline WT Stim 5' vs. Otof ${ }^{-1}$ Recov 5' (Stim 1') & $\mathrm{ns}$ & $>0.9999$ & \\
\hline Otof $^{-1-}$ Stim 5' vs. WT Recov 5' (Stim 1') & *** & $<0.0001$ & \\
\hline Otof ${ }^{-/-}$Stim 5' vs. Otof ${ }^{-/}$Recov 5' (Stim 1') & $\mathrm{ns}$ & $>0.9999$ & \\
\hline WT Recov 5' (Stim 1') vs. Otof ${ }^{-1-}$ Recov 5' (Stim 1') & *** & $<0.0001$ & \\
\hline
\end{tabular}


Figure $3.5 \mathrm{~B}$

\begin{tabular}{|l|c|c|c|}
\hline \multicolumn{1}{|c|}{ Genotype/Condition } & Mean \pm s.e.m. & N & n \\
\hline Rest & $100 \pm 11 \%$ & 3 & 100 \\
\hline Stim 1' & $234 \pm 13 \%$ & 1 & 37 \\
\hline Stim 5' & $438 \pm 38 \%$ & 2 & 52 \\
\hline BIM I + Stim 5' & $122 \pm 2 \%$ & 1 & 34 \\
\hline BIM I+KN-93+Stim 5' & $97 \pm 4 \%$ & 1 & 50 \\
\hline PMA 1' & $77 \pm 7 \%$ & 2 & 61 \\
\hline PMA 5' & $157 \pm 4 \%$ & 2 & 66 \\
\hline PMA 15' & $139 \pm 6 \%$ & 2 & 48 \\
\hline
\end{tabular}

\begin{tabular}{|c|c|c|c|}
\hline Compared group & $\begin{array}{l}\text { Statistical } \\
\text { significance }\end{array}$ & $P$-value & Statistical test \\
\hline \multicolumn{4}{|l|}{ PLA Otoferlin/P-Serine: } \\
\hline Rest vs. Stim 1' & $* * *$ & $<0.0001$ & \multirow{14}{*}{$\begin{array}{l}\text { Kruskal-Wallis test followed } \\
\text { by Dunn's multiple } \\
\text { comparison test }\end{array}$} \\
\hline Rest vs. Stim 5' & $* * *$ & $<0.0001$ & \\
\hline Stim 1' vs. Stim 5' & ns & $>0.9999$ & \\
\hline Stim 5' vs. BIM I + Stim 5' & $* *$ & 0.0013 & \\
\hline Stim 5' vs. BIM I+KN-93 + Stim 5' & $* * *$ & $<0.0001$ & \\
\hline $\begin{array}{l}\text { BIM I + Stim 5' vs. BIM I+KN-93 + Stim } \\
5^{\prime}\end{array}$ & $*$ & 0.0250 & \\
\hline Rest vs. BIM I + Stim 5 & * & 0.0133 & \\
\hline Rest vs. BIM I+KN-93 + Stim 5' & ns & $>0.9999$ & \\
\hline Rest vs. PMA 1' & ns & 0.3464 & \\
\hline Rest vs. PMA 5' & $* * *$ & $<0.0001$ & \\
\hline Rest vs. PMA 15' & $* * *$ & 0.0002 & \\
\hline PMA 1' vs. PMA 5' & $* * *$ & $<0.0001$ & \\
\hline PMA 1' vs. PMA 15' & $* * *$ & $<0.0001$ & \\
\hline PMA 5' vs. PMA 15' & ns & 0.1802 & \\
\hline
\end{tabular}

Figure $3.6 \mathrm{~B}$

\begin{tabular}{|l|c|c|c|}
\hline \multicolumn{1}{|c|}{ Genotype/Condition } & Mean \pm s.e.m. & N & n \\
\hline Rest & $100 \pm 5 \%$ & 2 & 43 \\
\hline Stim 1' & $138 \pm 3 \%$ & 2 & 44 \\
\hline Recov 5' (Stim 1') & $107 \pm 3 \%$ & 1 & 23 \\
\hline
\end{tabular}

\begin{tabular}{|c|c|c|c|}
\hline Compared group & $\begin{array}{l}\text { Statistical } \\
\text { significance }\end{array}$ & $P$-value & Statistical test \\
\hline \multicolumn{4}{|l|}{ Myosin VI immunofluorescence: } \\
\hline Rest vs. Stim 1' & *** & $<0.0001$ & \multirow{3}{*}{$\begin{array}{l}\text { Kruskal-Wallis test followed by } \\
\text { Dunn's multiple comparison test }\end{array}$} \\
\hline Rest vs. Recov 5' (Stim 1') & ns & $>0.9999$ & \\
\hline Stim 1' vs. Recov 5' (Stim 1') & $* * *$ & $<0.0001$ & \\
\hline
\end{tabular}


90 | Chapter 3

Figure $3.6 \mathrm{D}$

\begin{tabular}{|l|c|c|c|}
\hline \multicolumn{1}{|c|}{ Genotype/Condition } & Mean \pm s.e.m. & N & n \\
\hline Rest & $100 \pm 3 \%$ & 6 & 265 \\
\hline Stim 1' & $173 \pm 4 \%$ & 3 & 170 \\
\hline Recov 5' (Stim 1') & $183 \pm 4 \%$ & 3 & 153 \\
\hline BIM I + Stim 1' & $102 \pm 6 \%$ & 1 & 37 \\
\hline PMA 5' & $133 \pm 3 \%$ & 3 & 122 \\
\hline PMA 15' & $149 \pm 9 \%$ & 3 & 96 \\
\hline
\end{tabular}

\begin{tabular}{|c|c|c|c|}
\hline Compared group & $\begin{array}{l}\text { Statistical } \\
\text { significance }\end{array}$ & $P$-value & Statistical test \\
\hline \multicolumn{4}{|l|}{ PLA Otoferlin/Myosin VI: } \\
\hline Rest vs. Stim 1' & *** & $<0.0001$ & \multirow{15}{*}{$\begin{array}{c}\text { Kruskal-Wallis test followed } \\
\text { by Dunn's multiple } \\
\text { comparison test }\end{array}$} \\
\hline Rest vs. Recov 5' (Stim 1') & $* * *$ & $<0.0001$ & \\
\hline Rest vs. BIM I + Stim 1' & ns & $>0.9999$ & \\
\hline Rest vs. PMA 5' & *** & $<0.0001$ & \\
\hline Rest vs. PMA 15' & $* * *$ & $<0.0001$ & \\
\hline Stim 1' vs. Recov 5' (Stim 1') & ns & $>0.9999$ & \\
\hline Stim 1' vs. BIM I + Stim 1' & *** & $<0.0001$ & \\
\hline Stim 1' vs. PMA 5' & *** & $<0.0001$ & \\
\hline Stim 1' vs. PMA 15' & *** & $<0.0001$ & \\
\hline Recov 5' (Stim 1') vs. BIM I + Stim 1' & *** & $<0.0001$ & \\
\hline Recov 5' (Stim 1') vs. PMA 5' & $* * *$ & $<0.0001$ & \\
\hline Recov 5' (Stim 1') vs. PMA 15' & *** & $<0.0001$ & \\
\hline BIM I + Stim 1' vs. PMA 5' & $* *$ & 0.0013 & \\
\hline BIM I + Stim 1' vs. PMA 15' & $* * *$ & 0.0002 & \\
\hline PMA 5' vs. PMA 15' & ns & $>0.9999$ & \\
\hline
\end{tabular}

Figure 3.6F-G

\begin{tabular}{|l|c|c|c|c|}
\hline \multirow{2}{*}{ Genotype/Condition } & \multicolumn{2}{|c|}{ Mean \pm s.e.m. } & N & \multirow{2}{*}{. } \\
\cline { 2 - 3 } & Immunofluorescence & Apical/basal ratio & & \\
\hline Rest & $100 \pm 2 \%$ & $1.12 \pm 0.06$ & 3 & 89 \\
\hline Stim 1' & $163 \pm 8 \%$ & $0.83 \pm 0.04$ & 3 & 92 \\
\hline Recov 5' (Stim 1') & $118 \pm 2 \%$ & $1.19 \pm 0.08$ & 3 & 71 \\
\hline
\end{tabular}

\begin{tabular}{|c|c|c|c|}
\hline Compared group & $\begin{array}{l}\text { Statistical } \\
\text { significance }\end{array}$ & $P$-value & Statistical test \\
\hline \multicolumn{4}{|l|}{ Vglut3 immunofluorescence: } \\
\hline Rest vs. Stim 1' & *** & $<0.0001$ & \multirow{3}{*}{$\begin{array}{l}\text { Kruskal-Wallis test followed by } \\
\text { Dunn's multiple comparison test }\end{array}$} \\
\hline Rest vs. Recov 5' (Stim 1') & $* * *$ & $<0.0001$ & \\
\hline Stim 1' vs. Recov 5' (Stim 1') & $\mathrm{ns}$ & $>0.9999$ & \\
\hline \multicolumn{4}{|l|}{ Apical/basal Vglut3 ratio: } \\
\hline Rest vs. Stim 1' & *** & 0.0005 & \multirow{3}{*}{$\begin{array}{l}\text { Kruskal-Wallis test followed by } \\
\text { Dunn's multiple comparison test }\end{array}$} \\
\hline Rest vs. Recov 5' (Stim 1') & ns & $>0.9999$ & \\
\hline Stim 1' vs. Recov 5' (Stim 1') & *** & 0.0009 & \\
\hline
\end{tabular}


Figure 3.6I

\begin{tabular}{|l|c|c|c|}
\hline \multicolumn{1}{|c|}{ Genotype/Condition } & Mean \pm s.e.m. & N & n \\
\hline Rest & $100 \pm 2 \%$ & 5 & 78 \\
\hline Stim 1' & $104 \pm 1 \%$ & 4 & 146 \\
\hline Recov 5' (Stim 1') & $95 \pm 2 \%$ & 3 & 93 \\
\hline
\end{tabular}

\begin{tabular}{|l|c|c|c|}
\hline \multicolumn{1}{|c|}{ Compared group } & $\begin{array}{c}\text { Statistical } \\
\text { significance }\end{array}$ & P-value & Statistical test \\
\cline { 1 - 2 } PLA Otoferlin/Nglut3: & $\mathrm{ns}$ & 0.0961 & \multirow{2}{*}{$\begin{array}{c}\text { Kruskal-Wallis test followed by } \\
\text { Dunn's multiple comparison test }\end{array}$} \\
\cline { 1 - 3 } Rest vs. Stim 1' & $\mathrm{ns}$ & 0.5761 & \\
\hline Rest vs. Recov 5' (Stim 1') & ${ }^{* * *}$ & 0.0005 & \\
\cline { 1 - 2 } Stim 1' vs. Recov 5' (Stim 1') &
\end{tabular}

Figure 3.7B-C

\begin{tabular}{|l|c|c|c|c|}
\hline \hline \multirow{2}{*}{ Genotype/Condition } & \multicolumn{2}{|c|}{ Mean s.e.m. } & N & \multirow{2}{*}{$\mathbf{n}$} \\
\cline { 2 - 3 } & Immunofluorescence & Apical/basal ratio & & \\
\hline Rest & $100 \pm 1 \%$ & $1.04 \pm 0.02$ & 15 & 296 \\
\hline Stim 1' & $62 \pm 2 \%$ & $0.84 \pm 0.03$ & 11 & 174 \\
\hline Recov 5' (Stim 1') & $73 \pm 2 \%$ & $1.07 \pm 0.05$ & 8 & 141 \\
\hline BIM I + Stim 1' & $99 \pm 3 \%$ & $1.14 \pm 0.07$ & 1 & 26 \\
\hline
\end{tabular}

\begin{tabular}{|c|c|c|c|}
\hline Compared group & $\begin{array}{l}\text { Statistical } \\
\text { significance }\end{array}$ & $P$-value & Statistical test \\
\hline \multicolumn{4}{|l|}{ Calbindin immunofluorescence: } \\
\hline Rest vs. Stim 1' & *** & $<0.0001$ & \multirow{6}{*}{$\begin{array}{l}\text { Kruskal-Wallis test followed by } \\
\text { Dunn's multiple comparison test }\end{array}$} \\
\hline Rest vs. Recov 5' (Stim 1') & $* * *$ & $<0.0001$ & \\
\hline Rest vs. BIM I + Stim 1' & ns & $>0.9999$ & \\
\hline Stim 1' vs. Recov 5' (Stim 1') & $*$ & 0.0152 & \\
\hline Stim 1' vs. BIM I + Stim 1' & $* * *$ & $<0.0001$ & \\
\hline Recov 5' (Stim 1') vs. BIM I + Stim 1' & $* * *$ & $<0.0001$ & \\
\hline \multicolumn{4}{|l|}{ Apical/basal Calbindin ratio: } \\
\hline Rest vs. Stim 1' & *** & $<0.0001$ & \multirow{6}{*}{$\begin{array}{l}\text { Kruskal-Wallis test followed by } \\
\text { Dunn's multiple comparison test }\end{array}$} \\
\hline Rest vs. Recov 5' (Stim 1') & ns & 0.6380 & \\
\hline Rest vs. BIM I + Stim 1' & ns & 0.8940 & \\
\hline Stim 1' vs. Recov 5' (Stim 1') & $* * *$ & 0.0007 & \\
\hline Stim 1' vs. BIM I + Stim 1' & $* * *$ & 0.0001 & \\
\hline Recov 5' (Stim 1') vs. BIM I + Stim 1' & ns & 0.1856 & \\
\hline
\end{tabular}


92 | Chapter 3

Figure 3.7D

\begin{tabular}{|c|c|c|c|c|}
\hline \multirow[t]{2}{*}{ Genotype/Condition } & \multicolumn{2}{|c|}{ Mean \pm s.e.m. } & \multirow[t]{2}{*}{$\mathrm{N}$} & \multirow[t]{2}{*}{$\mathbf{n}$} \\
\hline & Calbindin & Otoferlin & & \\
\hline WT & $100 \pm 1 \%$ & $100 \pm 1 \%$ & 8 & 176 \\
\hline Otof ${ }^{\text {ISIST/IS15T }}$ & $72 \pm 3 \%$ & $42 \pm 1 \%$ & 3 & 83 \\
\hline Otof Pgal Iga & $92 \pm 2 \%$ & $30 \pm 1 \%$ & 4 & 76 \\
\hline $\operatorname{Otof}^{+/}$ & $83 \pm 3 \%$ & $51 \pm 2 \%$ & 3 & 99 \\
\hline Otof ${ }^{-1}$ & $56 \pm 1 \%$ & $0 \pm 0 \%$ & 4 & 108 \\
\hline
\end{tabular}

\begin{tabular}{|c|c|c|c|}
\hline Compared group & $\begin{array}{l}\text { Statistical } \\
\text { significance }\end{array}$ & $P$-value & Statistical test \\
\hline \multicolumn{4}{|l|}{ Calbindin levels: } \\
\hline WT vs. Otof ${ }^{\text {I515TIIIIST }}$ & *** & $<0.0001$ & \multirow{10}{*}{$\begin{array}{l}\text { Kruskal-Wallis test followed by } \\
\text { Dunn's multiple comparison test }\end{array}$} \\
\hline WT vs. Otof Pgal/Pga & ns & 0.5900 & \\
\hline WT vs. Otof ${ }^{+/-}$ & $* * *$ & 0.0002 & \\
\hline WT vs. Otof ${ }^{-/-}$ & $* * *$ & $<0.0001$ & \\
\hline Otof I5IST/I515T vs. Otof $^{\text {Pgal/Pga }}$ & $*$ & 0.0124 & \\
\hline Otof ${ }^{\text {ISISTII515T vs. Otof }}{ }^{+/-}$ & ns & $>0.9999$ & \\
\hline Otof IS15TIIIIIT vs. Otof ${ }^{-/-}$ & * & 0.0186 & \\
\hline Otof ${ }^{\text {Pall/Pga }}$ vs. Otof $f^{+/-}$ & ns & $>0.9999$ & \\
\hline Otof ${ }^{\text {PgalPga }}$ vs. Otof ${ }^{-/}$ & $* * *$ & $<0.0001$ & \\
\hline Otof $^{+/}$vs. Otof ${ }^{-/}$ & $* * *$ & $<0.0001$ & \\
\hline \multicolumn{4}{|l|}{ Otoferlin levels: } \\
\hline WT vs. Otof ${ }^{1515 T 1 / 515 T}$ & $* * *$ & $<0.0001$ & \multirow{10}{*}{$\begin{array}{l}\text { Kruskal-Wallis test followed by } \\
\text { Dunn's multiple comparison test }\end{array}$} \\
\hline WT vs. Otof ${ }^{\text {PgalIga }}$ & $* * *$ & $<0.0001$ & \\
\hline WT vs. Otof $f^{+/-}$ & **** & $<0.0001$ & \\
\hline WT vs. Otof ${ }^{-/-}$ & $* * *$ & $<0.0001$ & \\
\hline Otof ${ }^{\text {I5I5T/IIII5T }}$ vs. Otof ${ }^{\text {PgalIga }}$ & ns & 0.4603 & \\
\hline Otof ${ }^{\text {IS15T/I515T vs. Otof }}$ Of- $^{+/}$ & $\mathrm{ns}$ & $>0.9999$ & \\
\hline Otof IS15TIIIIITT vs. Otof $^{-/-}$ & $* * *$ & $<0.0001$ & \\
\hline Otof $P_{\text {gal/Pga }}$ vs. Otof $f^{+/}$ & $* * *$ & 0.0006 & \\
\hline Otof $f^{\text {PaalPga }}$ vs. Otof ${ }^{--}$ & $* * *$ & $<0.0001$ & \\
\hline Otof $f^{+/-}$vs. Otof ${ }^{-/-}$ & $* * *$ & $<0.0001$ & \\
\hline
\end{tabular}


Figure $3.7 \mathrm{E}$

\begin{tabular}{|l|c|c|c|c|}
\hline \multirow{2}{*}{ Genotype/Condition } & \multicolumn{2}{|c|}{ Mean \pm s.e.m. } & N & \multirow{2}{*}{$\mathbf{n}$} \\
\cline { 2 - 3 } & Calbindin & Otoferlin & & \\
\hline WTB6 - AAV & $100 \pm 2 \%$ & $100 \pm 1 \%$ & 11 & 276 \\
\hline WTCD1B6F1 + AAV.eGFP & $94 \pm 3 \%$ & $104 \pm 4 \%$ & 8 & 168 \\
\hline WTCD1B6F1 + DualAAV-TS & $117 \pm 4 \%$ & $147 \pm 5 \%$ & 3 & 62 \\
\hline Otof ${ }^{-/}$CD1B6F1 + DualAAV-TS & $70 \pm 5 \%$ & $31 \pm 3 \%$ & 1 & 13 \\
\hline Otof $^{-/}$CD1B6F1 + DualAAV-Hyb & $63 \pm 3 \%$ & $29 \pm 2 \%$ & 5 & 64 \\
\hline Otof ${ }^{-/}$CD1B6F1 - AAV & $50 \pm 2 \%$ & $3 \pm 0 \%$ & 6 & 142 \\
\hline
\end{tabular}

\begin{tabular}{|c|c|c|c|}
\hline Compared group & $\begin{array}{c}\text { Statistical } \\
\text { significanc } \\
\text { e }\end{array}$ & $P$-value & Statistical test \\
\hline \multicolumn{4}{|l|}{ Calbindin levels: } \\
\hline $\begin{array}{c}\text { WTB6 - AAV vs. } \\
\text { WTCD1B6F1 + AAV.eGFP }\end{array}$ & ns & 0.36 & \multirow[t]{8}{*}{$\begin{array}{l}\text { Kruskal-Wallis test followed by } \\
\text { Dunn's multiple comparison test }\end{array}$} \\
\hline $\begin{array}{c}\text { WTB6 - AAV vs. } \\
\text { WTCD1B6F1 + DualAAV-TS }\end{array}$ & ** & 0.002 & \\
\hline $\begin{array}{c}\text { WTB6 - AAV vs. } \\
\text { Otof }{ }^{-/} \text {CD1B6F1 + DualAAV-TS }\end{array}$ & *** & $<0.0001$ & \\
\hline $\begin{array}{c}\text { WTB6 - AAV vs. } \\
\text { Otof }{ }^{--} \mathrm{CD} 1 \mathrm{~B} 6 \mathrm{~F} 1+\text { DualAAV-Hyb }\end{array}$ & $* * *$ & $<0.0001$ & \\
\hline $\begin{array}{c}\text { WTB6 }-\mathrm{AAV} \text { vs. } \\
\text { Otof }{ }^{-/} \mathrm{CD} 1 \mathrm{~B} 6 \mathrm{~F} 1-\mathrm{AAV}\end{array}$ & *** & $<0.0001$ & \\
\hline $\begin{array}{l}\text { Otof }{ }^{--} \mathrm{CD} 1 \mathrm{~B} 6 \mathrm{~F} 1+\text { DualAAV-TS vs. } \\
\text { Otof }{ }^{--} \mathrm{CD} 1 \mathrm{~B} 6 \mathrm{~F} 1+\text { DualAAV-Hyb }\end{array}$ & ns & 0.38 & \\
\hline $\begin{array}{c}\text { Otof }^{--} \mathrm{CD} 1 \mathrm{~B} 6 \mathrm{~F} 1+\text { DualAAV-TS vs. } \\
\text { Otof }{ }^{--} \mathrm{CD} 1 \mathrm{~B} 6 \mathrm{~F} 1-\mathrm{AAV}\end{array}$ & ** & 0.0095 & \\
\hline $\begin{array}{c}\text { Otof }^{-/} \mathrm{CD} 1 \mathrm{~B} 6 \mathrm{~F} 1+\text { DualAAV-Hyb vs. } \\
\text { Otof }{ }^{--} \mathrm{CD} 1 \mathrm{~B} 6 \mathrm{~F} 1-\mathrm{AAV}\end{array}$ & $*$ & 0.0292 & \\
\hline \multicolumn{4}{|l|}{ Otoferlin levels: } \\
\hline $\begin{array}{c}\text { WTB6 - AAV vs. } \\
\text { WTCD1B6F1 + AAV.eGFP }\end{array}$ & ns & 0.58 & \multirow[t]{8}{*}{$\begin{array}{l}\text { Kruskal-Wallis test followed by } \\
\text { Dunn's multiple comparison test }\end{array}$} \\
\hline $\begin{array}{c}\text { WTB6 - AAV vs. } \\
\text { WTCD1B6F1 + DualAAV-TS }\end{array}$ & *** & $<0.0001$ & \\
\hline $\begin{array}{c}\text { WTB6 - AAV vs. } \\
\text { Otof }{ }^{--} \text {CD1B6F1 + DualAAV-TS }\end{array}$ & *** & $<0.0001$ & \\
\hline $\begin{array}{c}\text { WTB6 - AAV vs. } \\
\text { Otof }{ }^{--} \text {CD1B6F1 + DualAAV-Hyb }\end{array}$ & *** & $<0.0001$ & \\
\hline $\begin{array}{c}\text { WTB6 - AAV vs. } \\
\text { Otof }{ }^{-/} \text {CD1B6F } 1-\mathrm{AAV}\end{array}$ & $* * *$ & $<0.0001$ & \\
\hline $\begin{array}{l}\text { Otof }{ }^{--} \mathrm{CD} 1 \mathrm{~B} 6 \mathrm{~F} 1+\text { DualAAV-TS vs. } \\
\text { Otof } f^{--} \mathrm{CD} 1 \mathrm{~B} 6 \mathrm{~F} 1+\text { DualAAV-Hyb }\end{array}$ & ns & $>0.9999$ & \\
\hline $\begin{array}{c}\text { Otof }^{--} \mathrm{CD} 1 \mathrm{~B} 6 \mathrm{~F} 1+\text { DualAAV-TS vs. } \\
\text { Otof }{ }^{--} \mathrm{CD} 1 \mathrm{~B} 6 \mathrm{~F} 1-\mathrm{AAV}\end{array}$ & *** & $<0.0001$ & \\
\hline $\begin{array}{c}\text { Otof }^{--} \text {CD1B6F1 + DualAAV-Hyb vs. } \\
\text { Otof }{ }^{-/} \mathrm{CD} 1 \mathrm{~B} 6 \mathrm{~F} 1-\mathrm{AAV}\end{array}$ & $* * *$ & $<0.0001$ & \\
\hline
\end{tabular}


94 | Chapter 3

Figure $3.7 \mathrm{H}$

\begin{tabular}{|l|c|c|c|}
\hline \multicolumn{1}{|c|}{ Genotype/Condition } & Mean \pm s.e.m. & N & n \\
\hline Rest & $100 \pm 2 \%$ & 7 & 327 \\
\hline Stim 1' & $560 \pm 26 \%$ & 4 & 168 \\
\hline Recov 5' (Stim 1') & $77 \pm 5 \%$ & 2 & 107 \\
\hline BIM I + Stim 1' & $101 \pm 3 \%$ & 2 & 98 \\
\hline PMA 5' & $175 \pm 4 \%$ & 3 & 114 \\
\hline PMA 15' & $161 \pm 3 \%$ & 3 & 127 \\
\hline
\end{tabular}

\begin{tabular}{|c|c|c|c|}
\hline Compared group & $\begin{array}{c}\text { Statistical } \\
\text { significance }\end{array}$ & $P$-value & Statistical test \\
\hline \multicolumn{4}{|l|}{ PLA Otoferlin/Calbindin: } \\
\hline Rest vs. Stim 1' & $* * *$ & $<0.0001$ & \multirow{15}{*}{$\begin{array}{l}\text { Kruskal-Wallis test followed by } \\
\text { Dunn's multiple comparison test }\end{array}$} \\
\hline Rest vs. Recov 5' (Stim 1') & $* * *$ & $<0.0001$ & \\
\hline Rest vs. PMA 5' & *** & $<0.0001$ & \\
\hline Rest vs. PMA 15' & $* * *$ & $<0.0001$ & \\
\hline Rest vs. BIM I + Stim 1' & ns & $>0.9999$ & \\
\hline Stim 1' vs. Recov 5' (Stim 1') & $* * *$ & $<0.0001$ & \\
\hline Stim 1' vs. PMA 5' & $* * *$ & $<0.0001$ & \\
\hline Stim 1' vs. PMA 15' & *** & $<0.0001$ & \\
\hline Stim 1' vs. BIM I + Stim 1' & $* * *$ & $<0.0001$ & \\
\hline Recov 5' (Stim 1') vs. PMA 5' & $* * *$ & $<0.0001$ & \\
\hline Recov 5' (Stim 1') vs. PMA 15' & *** & $<0.0001$ & \\
\hline Recov 5' (Stim 1') vs. BIM I + Stim 1' & *** & 0.0006 & \\
\hline PMA 5' vs. PMA 15' & ns & $>0.9999$ & \\
\hline PMA 5' vs. BIM I + Stim 1' & *** & $<0.0001$ & \\
\hline PMA 15' vs. BIM I + Stim 1' & *** & $<0.0001$ & \\
\hline
\end{tabular}

Figure EV4

\begin{tabular}{|l|c|c|c|}
\hline \multicolumn{1}{|c|}{ Genotype/Condition } & Mean \pm s.e.m. & N & n \\
\hline Rest & $100 \pm 6 \%$ & 2 & 75 \\
\hline Stim 1' & $158 \pm 5 \%$ & 2 & 94 \\
\hline Recov 5' (Stim 1') & $82 \pm 6 \%$ & 2 & 61 \\
\hline
\end{tabular}

\begin{tabular}{|c|c|c|c|}
\hline Compared group & $\begin{array}{l}\text { Statistical } \\
\text { significance }\end{array}$ & $P$-value & Statistical test \\
\hline \multicolumn{4}{|l|}{ PLA PKC $\alpha / C a l b i n d i n:$} \\
\hline Rest vs. Stim 1' & *** & $<0.0001$ & \multirow{3}{*}{$\begin{array}{l}\text { Kruskal-Wallis test followed by } \\
\text { Dunn's multiple comparison test }\end{array}$} \\
\hline Rest vs. Recov 5' (Stim 1') & ** & 0.0051 & \\
\hline Stim 1' vs. Recov 5' (Stim 1') & *** & $<0.0001$ & \\
\hline
\end{tabular}


Appendix Table S2. Prediction of PKC phosphorylation sites in otoferlin.

\begin{tabular}{|c|c|c|c|c|}
\hline Tool & Model & Sites & & Reference \\
\hline NetPhos 3.1 & ANN & $\begin{array}{l}\text { T24; S55; S74; S173; S224; T229; } \\
\text { S230; T285; S319; T342; T466; S467; } \\
\text { S530; S541; S563; S683; S715; S784; } \\
\text { T904; T954; T1050; S1224; T1457; } \\
\text { T1504; T1538; T1577; T1597; S1646; } \\
\text { T1840; S1859; T1940 }\end{array}$ & $\frac{\frac{\mathrm{http}: / / \text { www.cbs.dtu }}{\mathrm{dk} / \text { services/NetPh }}}{\underline{\text { os-3.1/ }}}$ & $\begin{array}{c}\text { (Blom et al, } \\
\text { 2004) }\end{array}$ \\
\hline $\begin{array}{c}\text { KinasePhos } \\
1.0\end{array}$ & HMM & $\begin{array}{l}\text { T9; S74; S158; T159; S162; S166; } \\
\text { S183; S185; S237; S246; T285; T331; } \\
\text { T446; S467; S520; S541; S563; S685; } \\
\text { T750; S775; S784; S785; T787; S810; } \\
\text { T819; S866; T904; S970; T1083; } \\
\text { S1130; S1223; S1224; T1242; T1261; } \\
\text { S1293; T1318; T1416; T1424; T1482; } \\
\text { T1538; S1566; T1577; T1597; T1624; } \\
\text { T1639; T1756; S1789; S1796; T1809 }\end{array}$ & $\begin{array}{l}\text { http://kinasephos. } \\
\text { mbc.nctu.edu.tw/ }\end{array}$ & $\begin{array}{l}\text { (Huang et } \\
\text { al, 2005) }\end{array}$ \\
\hline PSSP & BDT & $\begin{array}{l}\text { T24; S29; S55; S74; S113; S145; S158; } \\
\text { S162; S166; S185; S233; S237; S246; } \\
\text { S286; S290; S335; S434; S467; S472; } \\
\text { S501; S520; S541; S563; S753; S775; } \\
\text { S784; S785; S796; S803; S810; T819; } \\
\text { T826; S918; S1099; S1130; S1223; } \\
\text { S1224; S1293; S1351; S1355; S1436; } \\
\text { S1566; S1579; S1705; S1789; S1793; } \\
\text { T1840; S1933 }\end{array}$ & $\frac{\text { http://ppsp.biocuck }}{\text { oo.org/ }}$ & $\begin{array}{c}\text { (Xue et al, } \\
\text { 2006) }\end{array}$ \\
\hline GPS 3.0 & PSSM, GA & $\begin{array}{l}\text { T24; S55; S74; S158; S162; S166; S185; } \\
\text { S219; S233; S467; S775; S777; S784; } \\
\text { S785; S796; S810; T819; S918; S1040; } \\
\text { S1099; S1224; S1351; S1355; S1965 }\end{array}$ & $\begin{array}{l}\text { http://gps.biocucko } \\
\text { o.org/online.php }\end{array}$ & $\begin{array}{l}\text { (Xue et al, } \\
\text { 2011) }\end{array}$ \\
\hline
\end{tabular}

Data information: Mouse otoferlin isoform 4 (NCBI accession number NP_001300696.1) was used for predictions. ANN, artificial neural network; HMM, Hidden Markov Models; PSSM, position-specific scoring matrices; GA, genetic algorithm; BDT, Bayesian decision theory. 


\subsection{Complementary studies}

\subsubsection{Exploring the possible interaction of otoferlin with other calcium buffer proteins}

In the previous chapter (chapter 3.3), I show that calbindin interacts with otoferlin upon strong IHC depolarization and PKC activation, possibly regulating endocytic events in murine IHCs.

While screening for potential interaction partners of otoferlin, other two calcium buffer proteins were identified as positive hints. A PLA between otoferlin and parvalbumin (Figure 3.8A) and between otoferlin and calretinin (Figure 3.8B) performed in explanted organs of Corti of WT B6 P14-16 mice in HBSS with $\mathrm{Ca}^{2+}$ resulted in strong fluorescent puncta distributed throughout the cytoplasm of the IHCs. These results indicate that these two calcium buffer proteins are in close proximity to otoferlin and might interact with it.
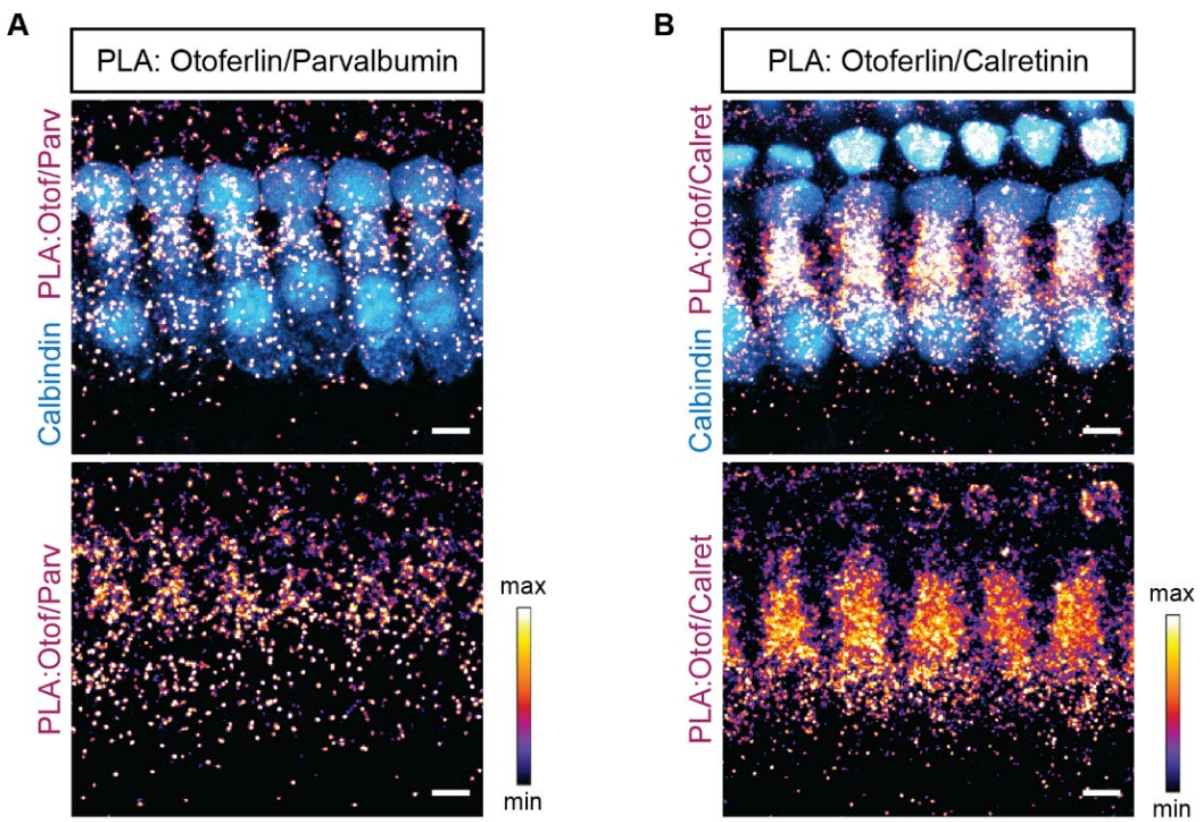

Figure 3.8. Proximity ligation assays for otoferlin and other calcium buffer proteins.

A High magnification views of a representative PLA for otoferlin and parvalbumin performed on WT B6 P15 mouse IHCs.

B High magnification views of a representative PLA for otoferlin and calretinin performed on WT B6 P15 mouse IHCs.

Data information: Calbindin (blue) was used as IHC marker. PLA channel is depicted with an intensity-coded lookup table with warmer colors representing higher pixel intensities. In (A-B), maximum intensity projections of confocal optical sections. Scale bars: $5 \mu \mathrm{m}$. IHC, inner hair cell. PLA: proximity ligation assay. Otof, otoferlin. Parv, parvalbumin. Calret, calretinin. 
Parvalbumin and calretinin levels also appear to differ among several otoferlin mutants. In Otof ${ }^{-/}$IHCs parvalbumin immunofluorescence levels were reduced to about $50 \%$ of WT levels (Otof ${ }^{-/}: 54 \pm 4 \%, \mathrm{n}=74$ IHCs vs. WT: $100 \pm 1 \%, \mathrm{n}=197$ IHCs; $\left.{ }^{* * *} P<0.0001\right)$, IHCs of Otof ${ }^{+/}$ mice showed a reduction of $-30 \%\left(\right.$ Otof $^{+-}: 68 \pm 2 \%, \mathrm{n}=127$ IHCs; $\left.{ }^{* * *} P<0.0001\right)$ and those of

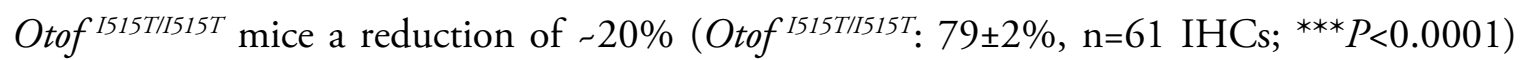
(Kruskal-Wallis test followed by Dunn's multiple comparison test; Figure 3.9). Calretinin immunofluorescence levels were also reduced in $\mathrm{Otof}^{-/-}$IHCs though to a less extent $\left(\right.$Otof $^{-1}$ : $77 \pm 1 \%, \mathrm{n}=74$ IHCs vs. WT: $100 \pm 1 \%, \mathrm{n}=98$ IHCs; $\left.{ }^{* * *} P<0.0001\right)$ but unaltered in Otof

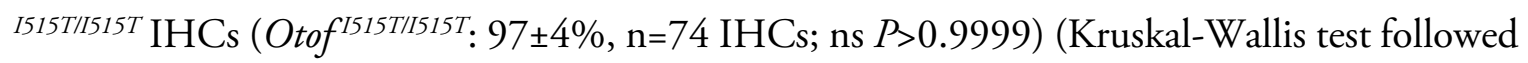
by Dunn's multiple comparison test; Figure 3.10.). These data support a role for parvalbumin and calretinin in IHC synaptic function, potentially mediating exocytic and/or endocytic processes.

A

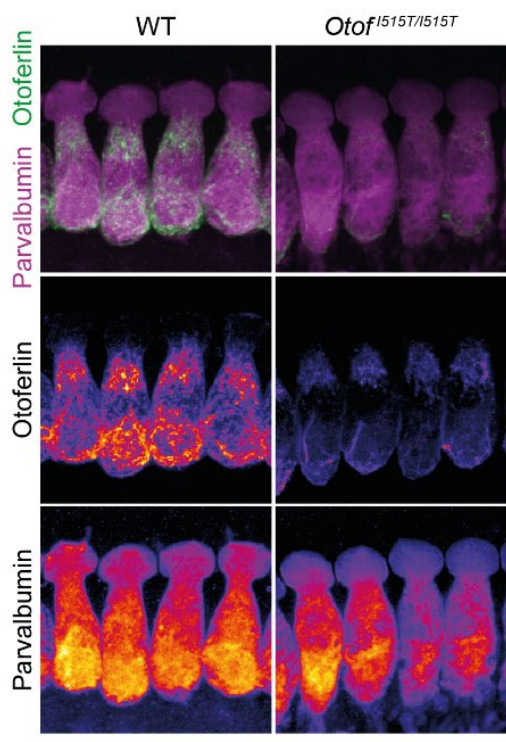

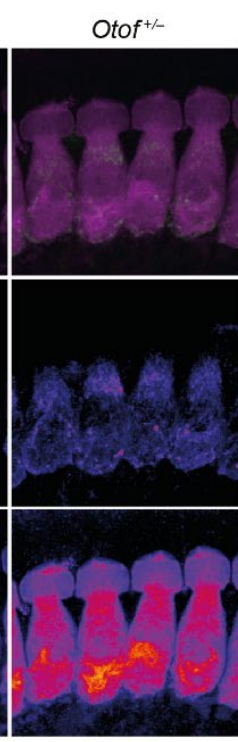

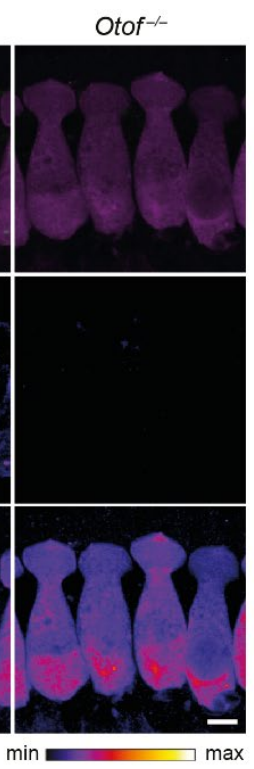

B

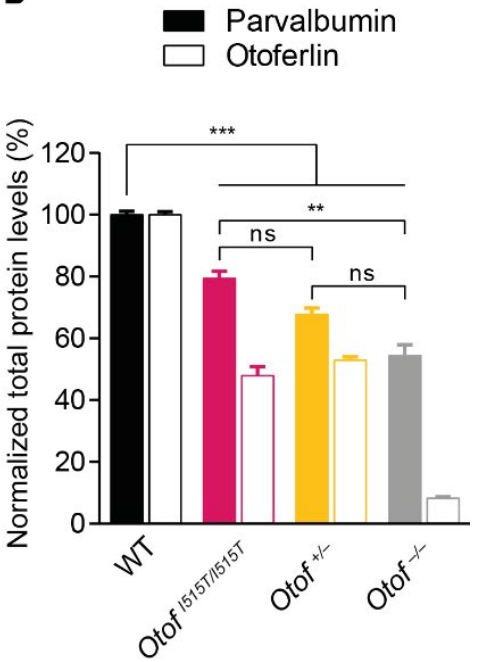

Figure 3.9. Parvalbumin immunofluorescence levels in different otoferlin mutant mouse lines.

A High magnification views of representative WT B6, Otof I515TIIIIST $^{\text {Otof }}{ }^{+/}$and Otof $f^{-/}$P14-P16 IHCs immunolabeled for parvalbumin and otoferlin, used for quantification of parvalbumin levels in B.

B Average parvalbumin and otoferlin immunofluorescence levels in mutant and WT IHCs (P14-16). Immunofluorescence levels were normalized to WT levels for each antibody separately. Data are displayed as mean \pm s.e.m.; ns $P>0.05,{ }^{*} P \leq 0.05,{ }^{* *} P \leq 0.01,{ }^{* * *} P \leq 0.001$ (Kruskal-Wallis test followed by Dunn's multiple comparison test); mean averages, sample size and statistical analysis are detailed in Appendix Supplementary Table 1.

Data information: In (A), maximum intensity projections of confocal optical sections. Scale bars: $5 \mu \mathrm{m}$. IHC, inner hair cell. 
Although exploring a potential role of these two calcium buffers in IHC exo- and endocytosis via interaction with otoferlin would be of interest, to follow up on these leads was beyond the scope of the current project. In any case, my data suggests that otoferlin might not act alone to mediate $\mathrm{Ca}^{2+}$-dependent exocytic and endocytic events in IHCs and seems to rather join forces with other $\mathrm{Ca}^{2+}$-binding proteins like PKC $\alpha$ and calbindin (this thesis; Cepeda et al, 2019), and possibly parvalbumin and calretinin.

A

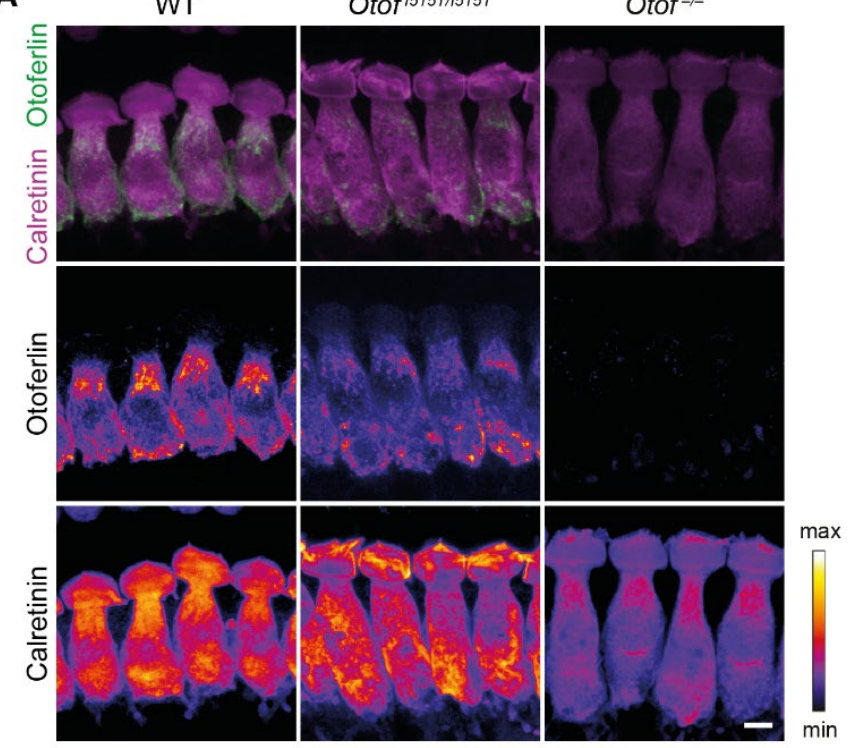

B

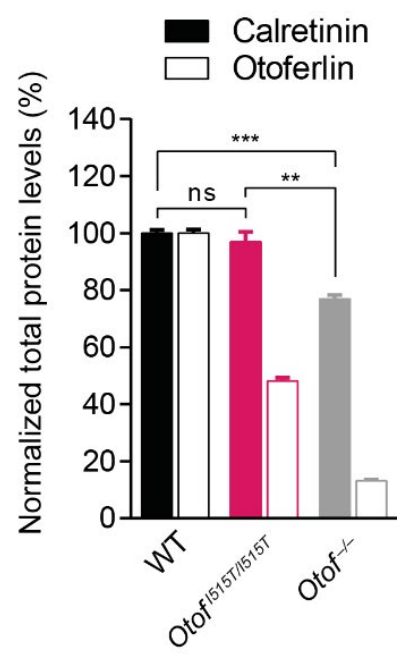

Figure 3.10. Calretinin immunofluorescence levels in different otoferlin mutant mouse lines.

A High magnification views of representative WT B6, Otof ${ }^{\text {I5I5TIIII5T }}$ and Otof ${ }^{-/}$P14-P16 IHCs immunolabeled for calretinin and otoferlin, used for quantification of calretinin levels in B.

B Average calretinin and otoferlin immunofluorescence levels in mutant and WT IHCs (P14-16). Immunofluorescence levels were normalized to WT levels for each antibody separately. Data are displayed as mean \pm s.e.m.; ns $P>0.05,{ }^{*} P \leq 0.05,{ }^{* *} P \leq 0.01$, ${ }^{* * *} P \leq 0.001$ (Kruskal-Wallis test followed by Dunn's multiple comparison test); mean averages, sample size and statistical analysis are detailed in Appendix Supplementary Table 1.

Data information: In (A), maximum intensity projections of confocal optical sections. Scale bars: $5 \mu \mathrm{m}$. IHC, inner hair cell.

PLA assays and quantification of protein levels were performed as described in Materials and Methods section of Cepeda et al (2019). Additional information:

Primary antibodies used in PLAs: mouse anti-otoferlin [13A9] (\#ab53233, Abcam, 1:500), rabbit anti-parvalbumin (\#ab11427, Abcam, 1:500), rabbit anti-calretinin (\#7697, Swant, 1:500), goat anti-calbindin D28k [C-20] (\#sc-7691, Santa Cruz Biotechnology, 1:100).

Primary antibodies used in immunostainings: mouse anti-otoferlin [13A9] (\#ab53233, Abcam, 1:300), rabbit anti-parvalbumin (\#ab11427, Abcam, 1:300), rabbit anti-calretinin (\#7697, Swant, 1:500). Secondary antibodies reported in Cepeda et al (2019). 
Chapter 4: General Discussion 

Hearing loss is one of the most common sensory deficiencies among the human population. About 466 million people worldwide suffer from disabling hearing loss $(6.1 \%$ of the world's population) and this number is estimated to reach over 900 million by 2050 (World Health Organization Webpage: https://www.who.int/deafness/estimates/en/). Hearing loss can be inherited (hereditary non-syndromic hearing loss, NSHL) or acquired through exposure to risk factors (Varga et al, 2003; Matsunaga et al, 2012; Shearer \& Smith, 2015; Nishio \& Usami, 2017). About 75 genes have been linked to a particular case of deafness, autosomal recessive non-syndromic hearing loss (DFNB) (Hereditary Hearing Loss Webpage: http://hereditaryhearingloss.org/), a sensorineural type of deafness which affects the inner ear or the auditory nerve. Pathogenic mutations in the OTOF gene, encoding the protein otoferlin, contribute to $2.3-10 \%$ of the cases of NSHL, and cause congenital prelingual autosomal recessive non-syndromic hearing loss 9 (DFNB9) in humans (Yasunaga et al, 1999; Varga et al, 2003; Shearer \& Smith, 2015), with effects ranging from moderate-to-profound depending on the OTOF mutation.

Otoferlin is a large multi- $\mathrm{C}_{2}$ domain protein, belonging to the ferlin family of membranefusion proteins (Lek et al, 2012). Mutations in the OTOF gene lead to deafness due to disruption of synaptic transmission between IHCs and the SGNs of the auditory nerve (auditory synaptopathy) (Roux et al, 2006, 200; Moser et al, 2013; Moser \& Starr, 2016). In analogy to synaptotagmins in the conventional synapse, otoferlin was initially proposed to act as the $\mathrm{Ca}^{2+}$ sensor for vesicle fusion based on functional studies with otoferlin knock-out mouse mutants (Roux et al, 2006) and $\mathrm{Ca}^{2+}$-binding studies via biochemical approaches (Johnson \& Chapman, 2010). Further studies confirmed the $\mathrm{Ca}^{2+}$ sensor hypothesis and additionally involved otoferlin in several steps of the synaptic vesicle cycle in IHCs including SV priming, SV fusion, SV tethering, SV reformation from bulk endosomes, SV replenishment to the ribbon, endocytosis and coupling of exo- and endocytosis by regulating active zone clearance (Pangrsic et al, 2010; Duncker et al, 2013; Vincent et al, 2014; Jung et al, 2015a; Strenzke et al, 2016; Meese et al, 2017; Michalski et al, 2017; Chakrabarti et al, 2018). Several proteins were identified as otoferlin interaction partners in different steps of the synaptic vesicle cycle (Roux et al, 2006; Heidrych et al, 2008, 2009; Ramakrishnan et al, 2009; Roux et al, 2009; Johnson \& Chapman, 2010; Zak et al, 2012; Duncker et al, 2013; Ramakrishnan et al, 2014; Vincent et al, 2014; Jung et al, 2015a; Hams et al, 2017; Meese et al, 2017) and helped understanding the role of otoferlin in IHC synaptic transmission. However, most studies were based on in vitro and static approaches, which can result in false-positive or false-negative interactions that would in any case have no physiological meaning.

This thesis has contributed to the efforts towards understanding the role of otoferlin in auditory IHCs' physiology. I provide insights into the mechanisms regulating otoferlin's function possibly in several steps of the synaptic vesicle cycle. I show that otoferlin can be phosphorylated by $\mathrm{Ca}^{2+} /$ calmodulin-dependent serine/threonine kinase delta (CaMKII $\delta$ ) and 
protein kinase $\mathrm{C}$ alpha $(\mathrm{PKC} \alpha)$ in an activity-dependent manner, and that phosphorylation can have repercussions in $\mathrm{Ca}^{2+}$-binding and membrane-binding properties, ultimately regulating otoferlin's involvement in various processes. The main findings and their implications are discussed in the following sections in detail.

\subsection{Activity-dependent regulation of the synaptic vesicle cycle}

Over the past decades, the molecular composition of the conventional presynapse and the steps of the synaptic vesicle cycle have been studied in detail, and great progress has been made in understanding the underlying molecular events. However, not much is known of how the synaptic vesicle cycle is regulated.

It is well established that calcium is fundamental to many biological processes, being essential to regulated exocytosis but also influencing endocytic retrieval processes at presynaptic terminals (reviewed in Neher \& Sakaba, 2008). Elevation of $\left[\mathrm{Ca}^{2+}\right]$ at presynaptic nerve terminals upon stimulation regulates neurotransmitter release. Synaptotagmins are $\mathrm{Ca}^{2+}$ sensors expressed at the presynapse able to detect these changes in $\left[\mathrm{Ca}^{2+}\right]$. Syt 1 is present on synaptic vesicles of neurons and neuroendocrine cells (Matthew et al, 1981; Perin et al, 1990) and is the major $\mathrm{Ca}^{2+}$ sensor for evoked neurotransmitter release in neurons (Brose et al, 1992; Yoshihara \& Littleton, 2002; Geppert et al, 1994; Maximov \& Südhof, 2005) and neuroendocrine cells (Voets et al, 2001; Sørensen et al, 2003), triggering fast and synchronous $\mathrm{Ca}^{2+}$-mediated synaptic vesicle fusion. Syt2, is assumed to have similar functions in neurons but it is not expressed in neuroendocrine cells (Geppert et al, 1991). At least a dozen additional synaptotagmin isoforms exist. Four synaptotagmins (Syt1, 2, 7 and 9) account for nearly all transmitter exocytosis (Südhof, 2014). After exocytosis, endocytosis takes place to retrieve excess membrane from which new vesicles are generated, replenishing the vesicle pool and maintaining exocytosis, with different modes of endocytosis being evoked depending on the amount of $\mathrm{Ca}^{2+}$ influx (reviewed in Wu et al, 2007). Various forms of endocytosis with differences in speed, number and vesicle size require either different $\mathrm{Ca}^{2+}$ sensors or one versatile $\mathrm{Ca}^{2+}$ sensor. At least a dozen additional synaptotagmin isoforms were identified which together with calmodulin, constitute well-characterized $\mathrm{Ca}^{2+}$ sensors for endocytosis and membrane traffic events (reviewed in Wu et al, 2019).

$\mathrm{Ca}^{2+}$ also modulates the activity of kinases and phosphatases expressed in nerve terminals, with implications to the phosphorylation state of synaptic proteins. Reversible phosphorylation of proteins controls not only protein-protein interactions but also protein activity and subcellular localization (Hunter, 2007). Several lines of evidence have shown that at conventional synapses presynaptic proteins are reversibly phosphorylated, suggesting that protein phosphorylation plays a role in the regulation of synaptic transmission, and might constitute the basis for the profound plasticity and fast adaptation to a multitude of signals observed in many synapses. 
Phosphorylation of presynaptic proteins by second messenger-activated protein kinases has been shown to regulate synaptic transmission, with effects on presynaptic plasticity (refilling of the RRP of vesicles and synaptic strength), protein interactions within the release apparatus, endocytosis and trafficking events (Südhof, 1995; Turner et al, 1999; Haberman et al, 2005; Jong et al, 2016). Identifying protein kinases and their targets in nerve terminals, particularly those regulated by synaptic activity or intracellular $\left[\mathrm{Ca}^{2+}\right]$, has been critical to elucidating the molecular mechanisms underlying modulation of synaptic transmission.

While some progress has been made in understanding the role of protein kinases in synaptic transmission in conventional synapses, mechanisms regulating the function of presynaptic proteins in IHC ribbon synapses have not been studied to date. This is largely because presynaptic proteins expressed in conventional synapses and that are targets of these kinases seem to be absent in IHC ribbon synapses (see chapter 1.1.3.1). Instead, IHC synapses express the multifunctional protein otoferlin, structurally and functionally related to some of these proteins (Pangršič et al, 2012), which seems to take over at least partially their function. While otoferlin is attributed to act as the $\mathrm{Ca}^{2+}$ sensor for exocytosis in IHC synapses (Roux et al, 2006; Johnson \& Chapman, 2010; Michalski et al, 2017), its involvement in many other processes like SV priming, SV fusion, SV tethering, SV reformation, SV replenishment, endocytosis and coupling of exo- and endocytosis (Roux et al, 2006; Pangrsic et al, 2010; Duncker et al, 2013; Vincent et al, 2014; Jung et al, 2015a; Strenzke et al, 2016; Meese et al, 2017; Michalski et al, 2017; Chakrabarti et al, 2018), that extend beyond a mere $\mathrm{Ca}^{2+}$-sensing role like that of Syt 1 in conventional synapses, predicts a fine regulation by phosphorylation and dephosphorylation events. This kind of regulation could at least in part explain otoferlin's multifunctionality across the synaptic vesicle cycle. In this thesis, I studied the activity-dependent regulation of otoferlin's function by the protein kinases CaMKII $\delta$ and PKC $\alpha$ and explored possible downstream effects of otoferlin's phosphorylation, particularly in respect to interaction partners and synaptic vesicle recycling.

\subsection{The phosphorylation of otoferlin by CaMKII}

In pull-down assays with chicken utricles, Dr. Meike Herget (Stanford University) first identified CaMKII $\delta$ as an interaction partner of otoferlin (Meese et al, 2017). I showed via immunostainings and PCRs that CaMKII $\delta$ is the main CaMKII expressed in rodent IHCs, being present throughout the cell and at the synaptic region (Meese et al, 2017: Figures 1 and 2; this thesis: Figures Figure 2.1 and Figure 2.2). Co-immunoprecipitation assays performed by Dr. Meike Herget with recombinant otoferlin and CaMKII $\delta$ expressed in HEK293 cells further supported a direct interaction of the two proteins (Meese et al, 2017). An in situ PLA assay performed with explanted organs of Corti confirmed close proximity $(<40 \mathrm{~nm})$ between otoferlin and CaMKII $\delta$ upon strong stimulation (Meese et al, 2017: Figure 4; this thesis: 
Figure 2.3), which led to the hypothesis that CaMKIIS may phosphorylate otoferlin thereby regulating its function.

\subsubsection{Otoferlin is phosphorylated by CaMKII $\delta$ in an activity-dependent manner}

Another PLA, this time for otoferlin and phosphoserine residues, was performed to test whether otoferlin or proteins interacting with otoferlin are phosphorylated in IHCs, and if this phosphorylation depends on IHC stimulation (Meese et al, 2017: Figure 10; this thesis: Figure 2.4). We noted the presence of a few PLA puncta in resting conditions, and high $\mathrm{K}^{+}$stimulation resulted in more and brighter puncta, suggesting otoferlin and/or its associated proteins are phosphorylated upon stimulation. Pre-treatment with a CaMKII inhibitor, KN-93, blocked the stimulation-dependent increase in PLA signal to a large extent, confirming that the activitydependent phosphorylation of otoferlin or otoferlin interactors is at least partially attributed to CaMKII's action.

Phosphorylation of otoferlin by CaMKII $\delta$ was proven in vitro by incubating two otoferlin fragments (otoferlin- $\mathrm{C}_{2} \mathrm{ABC}$ and otoferlin- $\mathrm{C}_{2} \mathrm{DEF}$ ) with recombinant CaMKII $\delta$. Mass spectrometry analysis retrieved ten phosphorylation sites (P1 to P10) in otoferlin, five within $\mathrm{C}_{2}$ domains ( $\mathrm{P} 1$ in $\mathrm{C}_{2} \mathrm{C}, \mathrm{P} 3$ in $\mathrm{C}_{2} \mathrm{D}$ and $\mathrm{P} 8-\mathrm{P} 10$ in $\mathrm{C}_{2} \mathrm{~F}$ ) and the remaining within linker regions (Meese et al, 2017: Figure 7). Six of the phosphorylation sites are conserved among species (P1, P3, P8-P10 - in $\mathrm{C}_{2}$ domains; P2 - in a linker region between $\mathrm{C}_{2} \mathrm{C}$ and $\mathrm{C}_{2} \mathrm{D}$ ). Interestingly, of the phosphorylation sites found within $\mathrm{C}_{2}$ domains, only two - P8 and P10 located in the $\mathrm{C}_{2} \mathrm{~F}$ domain are conserved among several ferlin proteins, including dysferlin, myoferlin, Fer1L4 and Fer1L5, although P10 only partially (Meese et al, 2017: Figure 8). Even though the CaMKII consensus sequence R/K-X-X-S/T (White et al, 1998) is absent in many of the phosphorylation hotspots, other studies reported it not to be necessary for substrate phosphorylation (Ando et al, 1991; Sun et al, 1994).

In conventional presynaptic terminals, synapsin 1, synaptotagmin 1, syntaxin 1, SNAP-25, VAMP2, and Cav1.2-3 L-type calcium channels (Llinás et al, 1985, 1991; Greengard et al, 1993; Popoli, 1993; Fukunaga et al, 1995; Nielander et al, 1995; Ryan et al, 1996; Hirling \& Scheller, 1996; Turner et al, 1999; Ohyama et al, 2002; Abiria \& Colbran, 2010; Jenkins et al, 2010) are CaMKII substrates. While some presynaptic proteins, like synapsin 1, undergo phosphorylation upon depolarization, other proteins involved in clathrin-mediated endocytosis are dephosphorylated upon depolarization e.g. by the $\mathrm{Ca}^{2+}$-dependent phosphatase calcineurin, as is the case of dynamin, amphiphysin, synaptojanin and the adaptor protein AP180 (Liu et al, 1994; Cousin \& Robinson, 2001). However, phosphorylation of the presynaptic machinery seems not to specifically target $\mathrm{C}_{2}$ domains. A recent 
phosphoproteomics study assessed the phosphorylation status of presynaptic proteins in resting and stimulated nerve terminals (isolated rat brain synaptosomes) and for all $\mathrm{C}_{2}$ domain proteins differentially regulated upon depolarization, no phosphorylation sites were detected within $\mathrm{C}_{2}$ domains (Kohansal-Nodehi et al, 2016). Only in very few cases phosphorylation seems to target $\mathrm{C}_{2}$ domains: cytosolic phospholipase A2 (Gijón et al, 1999), a novel PKC from Aplysia (Pepio \& Sossin, 2001), synaptotagmin 4 (Roggero et al, 2005), and rice small $\mathrm{C}_{2}$ domain proteins (Kang et al, 2013). In the novel PKC's case, phosphorylation of its non-Ca ${ }^{2+}$-binding $\mathrm{C}_{2}$ domain seems to increase PKC's affinity to phospholipids, inducing its translocation to the membrane (Pepio \& Sossin, 2001). In these studies, the effects of phosphorylation in $\mathrm{Ca}^{2+}$ binding were never assessed, and therefore reports on regulation of $\mathrm{Ca}^{2+}$ affinity of $\mathrm{C}_{2}$ domains through phosphorylation are non-existent, which in the case of otoferlin might constitute a unique regulatory mechanism.

\subsubsection{Phosphorylation by CaMKII $\delta$ affects $\mathrm{Ca}^{2+}$ affinity of otoferlin's $\mathrm{C}_{2}$ domains}

$\mathrm{C}_{2}$ domains are $\mathrm{Ca}^{2+}$-binding motifs found in a vast array of proteins involved in signaling processes including membrane trafficking, generation of lipid-second messengers, activation of GTPases and control of protein phosphorylation (Nalefski \& Falke, 1996). They have a variety of ligands and substrates, like $\mathrm{Ca}^{2+}$, membrane phospholipids, inositol phosphates, and proteins. Since not all $\mathrm{C}_{2}$ domain proteins seem to bind or be regulated by $\mathrm{Ca}^{2+}$, some $\mathrm{C}_{2}$ domains most likely have a mere structural role within proteins, being involved in proteinprotein interactions or membrane binding.

It is currently disputed which of otoferlin's $\mathrm{C}_{2}$ domains bind $\mathrm{Ca}^{2+}$. Some studies reported that all $\mathrm{C}_{2}$ domains of otoferlin are able to bind $\mathrm{Ca}^{2+}$ in vitro (Johnson \& Chapman, 2010; Padmanarayana et al, 2014) with the exception of the $\mathrm{C}_{2} \mathrm{~A}$ domain, which is not able to bind $\mathrm{Ca}^{2+}$ due to the missing $\mathrm{Ca}^{2+}$-coordinating aspartates and a shorter loop 1 (Johnson \& Chapman, 2010; Helfmann et al, 2011; Ramakrishnan et al, 2014). In the $\mathrm{C}_{2} \mathrm{~B}$ domain only one aspartate is present at the $\mathrm{Ca}^{2+}$-binding pocket (Jiménez \& Bashir, 2007), which was deemed to be insufficient for $\mathrm{Ca}^{2+}$ binding. Although Johnson \& Chapman, 2010 initially reported $\mathrm{Ca}^{2+}$ binding for the $\mathrm{C}_{2} \mathrm{~B}$ domain, this domain did not bind $\mathrm{Ca}^{2+}$ in our hands (Meese, 2015; Meese et al, 2017). A similar situation was found for the $\mathrm{C}_{2} \mathrm{C}$ domain, with only three aspartate residues being present at the $\mathrm{Ca}^{2+}$-binding region. $\mathrm{No} \mathrm{Ca}^{2+}$ binding was detected via microscale thermophoresis (MST) for otoferlin's $\mathrm{C}_{2} \mathrm{C}$ domain for either otoferlin- $\mathrm{C}_{2} \mathrm{C}$ or otoferlin- $\mathrm{C}_{2} \mathrm{ABC}$ fragments (Meese et al, 2017: Figure 9A), contradicting previous experimental findings (Johnson \& Chapman, 2010; Padmanarayana et al, 2014). More recently, an otoferlin knock-in mouse model Otof ${ }^{C 2 C / C 2 C}$ was generated, carrying two missense mutations (Asp515Ala and Asp517Ala) in the $\mathrm{Ca}^{2+}$-binding pocket of the $\mathrm{C}_{2} \mathrm{C}$ domain and 
predicted to affect $\mathrm{Ca}^{2+}$ binding (Michalski et al, 2017). Although in this study the authors claim that the $\mathrm{C}_{2} \mathrm{C}$ domain is able to bind $\mathrm{Ca}^{2+}$, no biochemical or binding assays were performed. The observed effects in exocytic rates as a consequence of the mutation could simply be secondary and result for example from folding changes that impair the binding of otoferlin to another protein (e.g. CaMKII $\delta$, PKC $\alpha$, calbindin).

In Meese et al (2017), the $\mathrm{Ca}^{2+}$-binding properties of otoferlin's $\mathrm{C}_{2} \mathrm{C}$ and $\mathrm{C}_{2} \mathrm{~F}$ domains were assessed via MST (van den Bogaart et al, 2012) using recombinant otoferlin single or multiple $\mathrm{C}_{2}$ domains heterologously expressed in E. coli. The influence of CaMKII $\delta$ phosphorylation on $\mathrm{Ca}^{2+}$ affinity of otoferlin's $\mathrm{C}_{2} \mathrm{C}$ and $\mathrm{C}_{2} \mathrm{~F}$ domains was studied by generating phosphomimetics substitutions where serine and threonine residues targeted by phosphorylation were replaced by aspartates to mimic the negative change introduced by phosphorylation (Meese, 2015; Meese et al, 2017).

A closer look at the CaMKII $\delta$ phosphorylation hotspots in otoferlin's sequence aligned with that of different ferlins, revealed that the phosphorylated threonine at P1 in otoferlin's $\mathrm{C}_{2} \mathrm{C}$ domain is occupied by an aspartate residue (D) in dysferlin and myoferlin (Meese et al, 2017: Figure 8). These aspartate residues in dysferlin and myoferlin are located in the top loops of $\mathrm{C}_{2}$ domains in these proteins, and are positioned close to another aspartate residue predicted to coordinate $\mathrm{Ca}^{2+}$ (Jiménez \& Bashir, 2007). To address the influence of CaMKII $\delta$ phosphorylation on $\mathrm{Ca}^{2+}$ affinity of otoferlin's $\mathrm{C}_{2} \mathrm{C}$ domain, this threonine residue was mutated into an aspartate (T448D). For the phosphomimetic $\mathrm{C}_{2} \mathrm{C}$ domain an increase in MST signal was observed with an apparent dissociation constant $\left(\mathrm{K}_{\mathrm{D}}\right)$ of $8.7 \pm 2.8 \mathrm{mM}$ (Meese et al, 2017: Figure $9 \mathrm{~B}-\mathrm{C})$. $\left[\mathrm{Ca}^{2+}\right]$ within $\mathrm{Ca}^{2+}$ hotspots at IHC ribbon synapses is estimated to range from $>10 \mu \mathrm{M}$ to $>100 \mu \mathrm{M}$ (Roberts, 1994; Beutner et al, 2001; Wong et al, 2014), meaning such high $\mathrm{K}_{\mathrm{D}}$ for $\mathrm{C}_{2} \mathrm{C}$ 's phosphomimetic represents a rather low affinity for $\mathrm{Ca}^{2+}$ and hence does not guarantee that the phosphomimetic $\mathrm{C}_{2} \mathrm{C}$ domain binds $\mathrm{Ca}^{2+}$ in vivo. That being said, phosphorylation by CaMKII $\delta$ likely converts the $\mathrm{C}_{2} \mathrm{C}$ domain of otoferlin from a non-Ca ${ }^{2+}-$ binding domain into a $\mathrm{Ca}^{2+}$-binding domain, though with low affinity.

As already mentioned, retrieved CaMKII $\delta$ phosphorylation sites in otoferlin's $\mathrm{C}_{2} \mathrm{~F}$ domain seem to be conserved among different ferlin proteins (Meese et al, 2017: Figure 8). To assess the effect of phosphorylation on the $\mathrm{Ca}^{2+}$ affinity of otoferlin's $\mathrm{C}_{2} \mathrm{~F}$ domain, serine and threonine residues at CaMKII $\delta$ phosphorylation sites P8, P9 and P10 on otoferlin were substituted by aspartates $(\mathrm{S} 1777 \mathrm{D}, \mathrm{S} 1808 \mathrm{D}, \mathrm{T} 1860 \mathrm{D})$. The wild-type $\mathrm{C}_{2} \mathrm{~F}$ domain retrieved a $\mathrm{K}_{\mathrm{D}}$ of $402 \pm 54 \mu \mathrm{M}$ (Meese et al, 2017: Figure 9D). Such $\mathrm{K}_{\mathrm{D}}$ for $\mathrm{Ca}^{2+}$ binding of otoferlin's $\mathrm{C}_{2} \mathrm{~F}$ domain might seem high (hence, resulting in low $\mathrm{Ca}^{2+}$ affinity), but similar values were previously reported for this domain $\left(\mathrm{K}_{\mathrm{D}} \sim 267 \mu \mathrm{M}\right)$ (Ramakrishnan et al, 2014) and for Syt1's $\mathrm{C}_{2} \mathrm{~B}$ domain $\left(\mathrm{K}_{\mathrm{D}}-50-250 \mu \mathrm{M}\right)$ (Fernandez et al, 2001; Radhakrishnan et al, 2009; van den Bogaart et al, 2012). Moreover, in our study, $\mathrm{Ca}^{2+}$-binding assays were performed in the 
absence of phospholipidic membranes, whose presence typically results in an increased $\mathrm{Ca}^{2+}$ affinity of $\mathrm{C}_{2}$ domains (Brose et al, 1992; Johnson \& Chapman, 2010; Padmanarayana et al, 2014). Such studies reported higher $\mathrm{Ca}^{2+}$ affinities for otoferlin's $\mathrm{C}_{2} \mathrm{~F}$ domain, with a $\mathrm{K}_{\mathrm{D}}$ of 20-25 $\mu \mathrm{M}$ (Johnson \& Chapman, 2010; Padmanarayana et al, 2014). Phosphomimetic $\mathrm{C}_{2} \mathrm{~F}$ bound $\mathrm{Ca}^{2+}$ with lower affinity, with a $\mathrm{K}_{\mathrm{D}}$ of $6.7 \pm 7.2 \mathrm{mM}$, an overall 10 -fold affinity reduction (Meese et al, 2017: Figure 9F). Phosphorylation by CaMKII $\delta$ possibly reduces the affinity of the $\mathrm{C}_{2} \mathrm{~F}$ domain to $\mathrm{Ca}^{2+}$.

When a PLA for otoferlin and phosphoserine residues, indicative of overall phosphorylation of otoferlin or protein complexes of which otoferlin is part of, was performed under stimulatory conditions, the PLA puncta did not overlap with but were located in close proximity to the ribbons (Meese et al, 2017: Figure 10D; this thesis: Figure 2.4C). This result indicates that phosphorylation might play an important role in targeting otoferlin to the active zone in IHCs. It is also possible that the presence of negatively charged phospholipids like $\mathrm{PIP}_{2}$ may increase the $\mathrm{Ca}^{2+}$ affinity of otoferlin's $\mathrm{C}_{2}$ domains in a similar fashion to what was observed for Syt 1 and PKC (Brose et al, 1992; Guerrero-Valero et al, 2009; van den Bogaart et al, 2012). If fact, otoferlin was reported to interact with the membrane lipid $\mathrm{PIP}_{2}$ (Roux et al, 2006; Ramakrishnan et al, 2009; Padmanarayana et al, 2014). Direct interactions between $\mathrm{PIP}_{2}$ and otoferlin's $\mathrm{C}_{2} \mathrm{C}$ and $\mathrm{C}_{2} \mathrm{~F}$ domains in specific (Padmanarayana et al, 2014) seem to target these domains toward $\mathrm{PIP}_{2}$-bearing liposomes, used in this study to mimic phospholipidic membranes, and therefore the $\mathrm{C}_{2} \mathrm{C}$ and $\mathrm{C}_{2} \mathrm{~F}$ domains of otoferlin were assumed to target the protein to the presynaptic area. This membrane targeting seems to occur in a $\mathrm{Ca}^{2+}$-independent manner, and in the specific case of the $\mathrm{C}_{2} \mathrm{~F}$ domain, the $\mathrm{Ca}^{2+}$-binding loops appear to directly interact with the lipid bilayer. These data highlight the importance of the $\mathrm{C}_{2} \mathrm{C}$ and $\mathrm{C}_{2} \mathrm{~F}$ domains for otoferlin's $\mathrm{Ca}^{2+}$-binding ability and membrane localization and are in line with results obtained by our research group for two particular mouse mutants for the $\mathrm{C}_{2} \mathrm{C}$ and $\mathrm{C}_{2} \mathrm{~F}$ domains of otoferlin which display distinct degrees of IHC disfunction and hearing impairment: the Otof ${ }^{1515 T / 1515 T}$ and Otof ${ }^{\text {PgalPga }}$ mutants. The Otof ${ }^{\text {Pgal/Pga }}$ mutant, carrying the p.Asp1767Gly missense mutation in the $\mathrm{C}_{2} \mathrm{~F}$ domain (Schwander et al, 2007), is profoundly deaf and displays severely reduced IHC sustained exocytosis but unaltered fast exocytic rates (Pangrsic et al, 2010). The Otof ${ }^{1515 T / 515 T}$ mutant, carrying the human p.Ile515Thr missense mutation in the $\mathrm{C}_{2} \mathrm{C}$ domain (Mirghomizadeh et al, 2002; Varga et al, 2006), displays moderate hearing impairment (temperature-sensitive) with sustained IHC exocytosis at levels between those of wild-type and Otof ${ }^{\text {PgalPga }}$ (Strenzke et al, 2016).

The amino acid residue changes caused by the p.Ile515Thr and p.Asp1767Gly mutations are predicted to impact otoferlin's stability (Pangrsic et al, 2010; Strenzke et al, 2016). Replacement of isoleucine 515, a naturally hydrophobic residue predicted to be positioned at the $\mathrm{C}_{2} \mathrm{C}$ domain hydrophobic core, by a threonine residue probably renders the core less hydrophobic, and therefore affects the stability of the protein. The replacement of aspartate 
1767, a negatively charged and polar residue which generally prefers to be on the surface of proteins and is frequently involved in protein active or binding sites, by a glycine, a non-polar and rather unique and flexible amino acid with more hydrophobic properties, will most certainly have serious repercussions on protein function. Detailed studies on these two mutants have proven exactly that. While wild-type otoferlin is normally distributed throughout the cytoplasm of IHCs but also at the plasma membrane and endosomal structures (Roux et al, 2006; Pangrsic et al, 2010; Strenzke et al, 2016: Figures 1A and D, Appendix Figure S1A), and Otof ${ }^{-/}$IHC completely lack otoferlin (Roux et al, 2006; Reisinger et al, 2011; Strenzke et al,

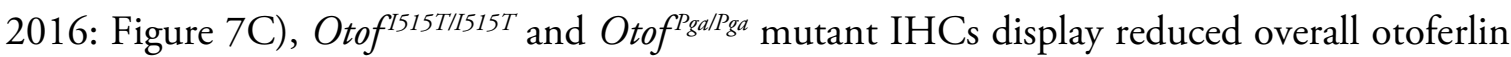
expression, $-31 \%$ and $-27 \%$ of wild-type levels, respectively (Strenzke et al, 2016: Figure 1G). Though overall otoferlin levels are comparable between Otof ${ }^{515 T / 1515 T}$ and Otof ${ }^{\text {Pgal/ga }}$ IHCs, otoferlin plasma membrane localization is distinct. While Otof ${ }^{1515 T / 1515 T}$ IHCs retain 35\% of membrane-bound otoferlin, Otof $f^{\text {PgalPga }}$ IHCs retain only 3\% of otoferlin at the basolateral plasma membrane (Strenzke et al, 2016: Figures 1D-F, 1I-J), which leads to the conclusion that the Otof ${ }^{\text {PgalPga }}$ mutation seems to affect otoferlin's membrane localization. Biolistic gene gun transfection of a $\mathrm{C}_{2} \mathrm{~F}$ deletion otoferlin cDNA construct into $\mathrm{Otof}^{-1-}$ IHCs led to hardly any otoferlin staining in the plasma membrane (Müller, 2017), supporting the importance of the $\mathrm{C}_{2} \mathrm{~F}$ domain for membrane localization. The Otof ${ }^{\text {PgalPga }}$ mutation appears to additionally interfere with the $\mathrm{Ca}^{2+}$ binding ability of the $\mathrm{C}_{2} \mathrm{~F}$ domain (Meese, 2015), potentially affecting the targeting of otoferlin towards the plasma membrane and other cellular compartments (Strenzke et al, 2016: Figure $1 \mathrm{H}$ ) and most likely affecting its interaction with proteins responsible for membrane retrieval like AP-2 (Duncker et al, 2013; Jung et al, 2015a). Additional implications of these mutations and the regulatory role by CaMKII $\delta$ are discussed later on in chapter 4.4 .

\subsection{The phosphorylation of otoferlin by PKCa}

With the goal of assessing the potential role of other kinases in IHC synaptic function, I screened for the presence of other kinases in IHCs.

\subsubsection{PKC $\alpha$ is expressed in IHCs and redistributes upon activation}

PKC $\alpha$ was found to be highly expressed in murine IHCs, with a homogeneous distribution throughout the cell and to a less extent at the plasma membrane (Figure EV1). To study the dynamics of PKC $\alpha$ activation in IHCs, I decided to apply an already-established resting/stimulation/recovery paradigm (Kamin et al, 2014; Revelo et al, 2014). In these two studies, trafficking events and SV recycling were followed using FM 1-43 dye and the mCLING probe to analyze endocytic intermediates and their conversion into SVs. The authors 
observed that in IHCs constitutive membrane trafficking is abundant and takes place at rest and during stimulation, and most endocytosed material is converted into tubular organelles in the top and nuclear areas of the cell giving rise to larger vesicles that resemble early endosomes. Upon stimulation, SVs tethered to the ribbons are released, and SV recycling takes place at the base of the cell in close proximity to the AZs. During recovery after the stimulation period, membrane endocytosis leads to the formation of large cisterns that later on are converted to small vesicles (see Figure 1.4).

Strong IHC depolarization via high $\mathrm{K}^{+}$stimulation $(1 \mathrm{~min}, 65 \mathrm{mM} \mathrm{KCl}, 2 \mathrm{mM} \mathrm{CaCl}$ ) led to the activation and strong expression of PKC $\alpha$ at the basolateral plasma membrane of IHCs and was characterized by accumulations of PKC $\alpha$ and otoferlin in common structures near the active zone (Figure 3.2). These structures did not overlap but were close to the synaptic ribbons and revealed to be larger than synaptic vesicles, resembling recycling endosomes previously described by several research groups (Kamin et al, 2014; Revelo et al, 2014; Watanabe et al, 2014; Jung et al, 2015a; Strenzke et al, 2016).

\subsubsection{PKC $\alpha$ interacts with and phosphorylates otoferlin}

To investigate a possible interaction between otoferlin and PKC $\alpha$ in IHCs, I first performed a PLA for otoferlin and PKC $\alpha$ in different conditions. A 4-fold increase in PLA signal from resting to strong stimulatory conditions indicated close proximity between the proteins during strong IHC stimulation (Figure 3.3A-B). Pull-down and co-IP assays later confirmed the interaction of the two proteins (Figure 3.3C-D).

I then performed an in vitro assay with heterologously expressed otoferlin and recombinant PKC $\alpha$ to retrieve possible phosphorylation sites in otoferlin. LC-MS/MS analysis was done in collaboration with Dr. Christof Lenz (Core Facility Proteomics, Institute of Clinical Chemistry, University Medical Center Göttingen; Bioanalytical Mass Spectrometry Group, Max Planck Institute for Biophysical Chemistry, Göttingen) and retrieved phosphorylation of otoferlin at five serine residues: S158, S775, S1169, S1224 and S1436 (otoferlin variant 4, NP_001300696.1) (Figure 3.3E-F; Appendix Figures Appendix Figure S3-Appendix Figure S10). All phosphorylation sites were found to be conserved between mammalian and nonmammalian otoferlin orthologs, with the exception of S1224 at the $\mathrm{C}_{2} \mathrm{de}$ domain only conserved among mammalian species (Appendix Figure S11). It is interesting to note that most phosphorylation sites were not located within otoferlin's $\mathrm{C}_{2}$ domains but rather in linker regions predicted to be involved in phospholipid-binding. Moreover, phosphorylation at $\mathrm{S} 775$ could possibly facilitate the interaction of FerA with membranes in the presence of $\mathrm{Ca}^{2+}$, as proposed before (Harsini et al, 2018), by changing the folding and/or lipid-binding properties 
of the domain through conversion from a four-helix bundle to an inverted hydrophobic membrane-associating structure.

\subsubsection{Activity-dependent phosphorylation of otoferlin and/or otoferlin- bearing complexes has an overall contribution of CaMKII $\delta$ and PKC $\alpha$}

A PLA between otoferlin and phosphoserine residues was used to test whether otoferlin and/or proteins interacting with otoferlin are phosphorylated in rat (Meese et al, 2017: Figure 10B) and mouse (this thesis: Figure 2.4) IHCs. Upon stimulation with high $\mathrm{K}^{+}$the PLA signal increased when compared to resting conditions, but this effect could be only partially blocked by the CaMKII inhibitor KN-93 (Meese et al, 2017: Figure 10B-C), suggesting the involvement of other kinases in the regulation of synaptic function through phosphorylation of otoferlin in mammalian IHCs. In this thesis, I showed that the stimulation-dependent increase in PLA signal can also be blocked to a large extent by the PKC $\alpha$ inhibitor BIM I, and it is completely blocked by treatment with inhibitors of both kinases (Figure 3.5). These results point to a combined action of CaMKII $\delta$ and PKC $\alpha$ in phosphorylating otoferlin or otoferlin interaction partners.

\subsubsection{Phosphorylation of otoferlin by PKC $\alpha$ promotes the interaction of otoferlin with myosin VI and calbindin}

Does the phosphorylation of otoferlin by PKC $\alpha$ promote the interaction of otoferlin with other proteins involved in SV recycling?

In this thesis, I show that the previously reported interaction of otoferlin with myosin VI (Heidrych et al, 2009; Roux et al, 2009) is PKCa-dependent. The PLA signal for the interaction otoferlin-myosin VI increased when compared to the resting condition, and this increase was in the same order of magnitude when $\mathrm{PKC} \alpha$ was activated either by high $\mathrm{K}^{+}$ stimulation or pharmacologically (Figure 3.6C-D). Treatment with the PKC $\alpha$ inhibitor fully abolished the stimulation-induced increase in PLA signal. These results suggest that otoferlin interacts with myosin VI in a PKC $\alpha$-dependent manner. This assumption is supported by the results obtained for the PLA pair otoferlin-Vglut3. In all conditions (resting, stimulation and recovery) the PLA was positive but the signal intensity did not change (Figure 3.6H-I), most likely because both proteins are known to localize to common structures in IHCs and therefore follow shared trafficking pathways without necessarily interacting. Although Vglut3 
immunofluorescence raised upon strong IHC stimulation, which was accompanied by a relocation of the protein to the basolateral plasma membrane (Figure 3.6E-G), this result likely reflects i) exposure of the epitope, ii) transport of distal SVs to the release sites, and iii) fusion of $\mathrm{SV}$ s with the $\mathrm{AZ}$ membrane during sustained release.

Changed calbindin immunofluorescence levels among different experimental conditions (decreased immunofluorescence upon stimulation; Figure 3.7A-C) and among several otoferlin mouse mutants (general decreased immunofluorescence; Figure 3.7D and Figure EV3A), and rescue of these levels in Otof ${ }^{-/}$IHCs when otoferlin was reintroduced via dual-AAV approaches (Figure 3.7E), led us to investigate a possible interaction of otoferlin with calbindin. A PLA between otoferlin and calbindin (Figure 3.7G-H) resulted in a >5-fold increase in signal upon strong IHC stimulation when compared to resting conditions, indicating close proximity between the two proteins upon stimulation. The stimulation-induced increase in PLA signal was fully blocked by a PKC $\alpha$ inhibitor. A pull-down assay confirmed physical association of the two proteins in vitro (Figure 3.7F). An additional PLA between calbindin and PKC $\alpha$ led to a not so pronounced increase in signal upon stimulation, possibly because the two proteins do not interact directly, but rather interact indirectly through a scaffolding protein, likely otoferlin. Given the strong activity-dependency of the interactions otoferlin-PKC $\alpha$ and otoferlin-calbindin, it is conceivable that the three proteins are part of one complex during stimulation, with PKC $\alpha$ and calbindin binding to distinct regions of otoferlin.

\subsubsection{PKC $\alpha$ 's probable role in otoferlin-dependent SV reformation events}

Multiple lines of evidence suggest that the structures where otoferlin and PKC $\alpha$ accumulate and interact are bulk endosomes and/or ultrafast endocytic compartments from where new SVs are reformed. When in an earlier study our group examined IHCs via electron microscopy, otoferlin immunogold particles were found in large membranous compartments positioned laterally to the active zones (Strenzke et al, 2016). Due to their size (ranging from $>50$ to -450 $\mathrm{nm}$ in diameter) and because they exhibited budding of clathrin-coated vesicles (Strenzke et al, 2016: Figure 7I, F,G) these structures were classified as endosomal recycling compartments. Structures about four times the size of synaptic vesicles and positioned laterally to active zones are associated with ultrafast endocytosis in hippocampal synapses (Watanabe et al, 2013), which given the similar morphology support the ultrafast endocytosis hypothesis in IHCs. Additional evidence strengthens this theory that PKC $\alpha$ and otoferlin might colocalize in such compartments involved in ultrafast endocytosis. Firstly, the lack of PKC $\alpha$ "clustering" in the vicinity of the ribbons when using milder stimulations ( $25 \mathrm{mM} \mathrm{KCl}, 2 \mathrm{mM} \mathrm{CaCl}_{2}$, for $1 \mathrm{~min}$ ) (Figure EV2A). Revelo and collaborators (Revelo et al, 2014) observed that milder stimulations (10 or $25 \mathrm{mM} \mathrm{KCl}, 2 \mathrm{mM} \mathrm{CaCl}_{2}$, for $1 \mathrm{~min}$ ) retrieved single vesicles from the plasma membrane via clathrin-mediated endocytosis but also gave rise to large organelles clearly 
separated from the plasma membrane in areas surrounding the synaptic ribbon (similar to bulk endosomes in conventional synapses). However, stronger stimulations $(65 \mathrm{mM} \mathrm{KCl}, 2 \mathrm{mM}$ $\mathrm{CaCl}_{2}$, for $1 \mathrm{~min}$ ) generated even larger membrane infoldings and cisterns continuous with the basolateral plasma membrane (Revelo et al, 2014: Figure 5A) - similar to membrane infoldings of stimulated IHCs observed by electron microscopy in Neef et al, 2014. Secondly, in strongly stimulated Otof ${ }^{-1}$ IHCs, PKC $\alpha$ distributed to endosomal compartments at the base of the cells (Figure 3.4D-G) like it happened in wild-type IHCs (Figure 3.1A-D), but while in wildtype IHCs the pronounced accumulations in endosomal structures disappeared for longer stimulation times and during recovery, in $\mathrm{Otof}^{-/}$IHCs PKC $\alpha$ remained at the basolateral plasma membrane and in endosomes (Figure 3.4D-E). Processes like exocytosis, endocytosis and SV reformation, proven to be dependent on otoferlin, are undoubtedly severely impaired in Otof ${ }^{-1}$ IHCs. Recycling endosomes might not be converted to SVs as fast as in the presence of otoferlin, and if we consider PKC $\alpha$ to be involved in this event, the delay in the whole process might explain why PKC $\alpha$ is still present in these compartments in $\mathrm{Otof}^{-/}$IHCs during longer stimulations and recovery. In fact, the clustering and redistribution of PKC $\alpha$ towards the basolateral plasma membrane and to endosomal compartments in IHCs is coherent with the behavior observed in other cell types upon its activation, where PKC is recruited to membranes (Hermelin et al, 1988; Huang et al, 1997; Feng et al, 1998, 2000; Tardif et al, 2002; González et al, 2003; Schechtman et al, 2004; Wu et al, 2006; Cordey \& Pike, 2006). In many cases, PKC $\alpha$ regulates the function of several cell surface receptors and membrane transporters by inducing their translocation from the plasma membrane to endocytic compartments (Peng et al, 2002; Le et al, 2002; Loder \& Melikian, 2003; Becker \& Hannun, 2003). In neuronal synapses, PKC is responsible for the trafficking of Syt XI to endocytic recycling compartments (Haberman et al, 2005), but is also involved in endocytic processes in general (reviewed in Alvi et al, 2007). The co-trafficking of PKC $\alpha$ and otoferlin towards such structures upon IHC stimulation points toward a possible interaction of the two proteins at this location, and to an involvement of PKC $\alpha$ in the regulation of endocytic processes via phosphorylation of otoferlin and/or other proteins.

As already mentioned, myosin VI-dependent trafficking events in IHCs seem to be dependent on PKC and otoferlin. A PLA between otoferlin and myosin VI led to a comparable increase in PLA signals upon PKC $\alpha$ activation via high $\mathrm{K}^{+}$stimulation or pharmacological activation, and treatment with a PKC $\alpha$ inhibitor abolished the stimulation-induced increase in PLA signal.

Myosin VI and F-actin are closely associated and affect the trafficking of cellular components (reviewed in Kneussel \& Wagner, 2013). F-actin was also shown to control otoferlindependent exocytosis in IHCs, forming dense cage-shape structures beneath the ribbon in order to maintain a tight special organization of calcium channels (Vincent et al, 2015) which likely interact with otoferlin (Ramakrishnan et al, 2009; Hams et al, 2017). Myosin VI is also 
involved in cargo sorting in the early endocytic pathway (Tumbarello et al, 2013). It is then conceivable that myosin VI and F-actin in association with otoferlin might be involved in PKC-dependent trafficking processes in IHCs, which most probably involve recycling of endocytic intermediates. Since otoferlin also interacts with calbindin in an activity-dependent manner, calbindin might also be involved in endocytic events, at least at some stage of the SV cycle.

\subsubsection{The otoferlin-calbindin interaction might be important for ultrafast endocytosis}

The observation that calbindin immunofluorescence levels are strongly reduced in Otof I515TII515T but not in Otof ${ }^{\text {PaalPga }}$ IHCs, although otoferlin levels are comparable in both mutants, might be inconsistent and incompatible with a direct impact of calbindin in the process. However, these two mutations were proposed to affect distinct cellular processes: SV replenishment to the ribbon in the case of the pachanga mutation, and SV reformation from endocytic recycling compartments in the case of the p.Ile515Thr mutation. Given our observations, it is anticipated that calbindin may be involved in only one of these processes via interaction with otoferlin. Calbindin seems not to be absolutely required for hearing nor it confers protection against moderate noise-induced hearing loss (Airaksinen et al, 2000). Supporting this finding, a triple knock-out mouse model of calbindin, parvalbumin and calretinin ( $\mathrm{Ca}^{2+}$ buffers) presented almost unaffected hearing abilities (Pangršič et al, 2015). Since susceptibility to noise is more likely to be induced in timescales ranging from minutes to hours and given the short timescale of the PKC $\alpha$-otoferlin-calbindin interaction (growing weaker between 1 and 5-minute strong depolarizations), it is probable that the mechanisms in which calbindin is taking part in might comprehend shorter periods.

We hypothesize that calbindin involvement in the complex might be the trigger for ultrafast endocytosis events. Patch-clamp capacitance measurements from calbindin knock-out IHCs (Pangršič et al, 2015) and actin polymerization studies (Vincent et al, 2015; Guillet et al, 2016; Tertrais et al, 2019) support these claims. For a comprehensive review of these studies, refer to the discussion section of Cepeda et al (2019) (chapter 3.3.5 of this thesis).

To be unequivocally sure on which processes PKC $\alpha$ (in a complex with otoferlin and calbindin) might be involved, one would need to perform patch-clamp capacitance measurements to monitor exo- and endocytosis rates in IHCs after treatment with PKC inhibitors. The main challenge here will be to find a paradigm where PKC is reliably activated. 


\subsubsection{Parvalbumin and calretinin might also interact with otoferlin to mediate $\mathrm{Ca}^{2+}$-dependent exocytic and endocytic events}

The contribution of other calcium buffer proteins like parvalbumin and calretinin to exocytic and/or endocytic events is not off the table. A PLA for otoferlin-parvalbumin and otoferlincalretinin was positive (Figure 3.8) and parvalbumin and calretinin immunofluorescence levels in different mutant IHCs were also altered (Figures Figure 3.9 and Figure 3.10) but to follow up on these leads was beyond the scope of the current project.

Parvalbumin levels were comparable with calbindin levels observed for these mutants (Figure 3.7D), while calretinin levels seemed to be affected to a lesser extent. Contrary to what happens in other cell types, hair cells (of most species) express the three $\mathrm{Ca}^{2+}$-binding proteins, possibly reflecting the need for buffers with different properties to act in different $\mathrm{Ca}^{2+}$ signalling mechanisms, which in these cells are not well spatially isolated. Even though the levels of parvalbumin and calretinin might be changed due to indirect effects like reduced vesicle turnover in these mutants, it is tempting to speculate about a potential involvement of the two buffers in exocytic and/or endocytic events. It is also possible that parvalbumin and calretinin are mediating distinct membrane retrieval events. For instance, it is noteworthy that Otof 1515TI515T IHCs show altered parvalbumin levels but no changes in calretinin levels were

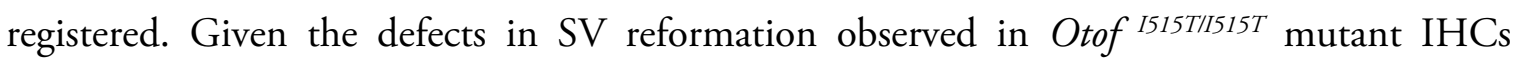
(Strenzke et al, 2016) induced by the p.Ile515Thr mutation in the $\mathrm{C}_{2} \mathrm{C}$ domain of otoferlin, one of the explanations can be that parvalbumin joins otoferlin to mediate the reformation of properly sized SVs from endocytic recycling compartments. If we consider otoferlin and parvalbumin to be interaction partners, the conformational changes in otoferlin induced by the p.Ile515Thr mutation might also increase the affinity between the two proteins, leaving the parvalbumin epitope to which the antibody binds to less exposed, and hence the decreased parvalbumin levels. In the case of calretinin, if we consider a scenario where defective otoferlin (p.Ile515Thr) is unable to bind $\mathrm{Ca}^{2+}$, and we assume calretinin to have also a sensor function (besides $\mathrm{Ca}^{2+}$ buffering), calretinin might be overexpressed in Otof ${ }^{1515 T / 1515 T}$ IHCs to compensate the lack of "good" otoferlin, hence the unchanged levels even though the reduced vesicle turnover in these cells. Another scenario that could explain the unchanged calretinin levels might be that this protein is mediating other processes like vesicle replenishment to the ribbon (a process affected in Otof ${ }^{\text {Pgal/ga }}$ IHCs) or other trafficking events. It would be interesting to quantify parvalbumin and calretinin levels also for Otof ${ }^{\text {Pgal/ga }}$ mutant IHCs (Pangrsic et al, 2010), which present SV replenishment defects, and see if they are unchanged as it was observed for calbindin levels. If changes would be observed, they could potentially point towards a role of these two proteins in mediating replenishment of SVs to the ribbon.

My data suggests that otoferlin might not be a solitary $\mathrm{Ca}^{2+}$ sensor for endocytic processes in IHCs but might rather act in consortium with other $\mathrm{Ca}^{2+}$-binding proteins (like PKC $\alpha$, 
calbindin, parvalbumin and calretinin). Otoferlin most likely interacts with different proteins to mediate not only exocytosis but also different modes of endocytosis and/or different steps of the SV cycle like SV reformation from large endosomes or SV replenishment to the ribbon.

\subsection{Potential impact of $O T O F$ mutations in otoferlin's regulation by protein kinases}

The function of a protein is largely determined by its three-dimensional structure. Mutations leading to changes in the amino acid sequence usually affect protein folding and ultimately result is overall stability perturbations. Changes in protein folding can, for instance, affect membrane targeting, interaction with other proteins, cofactor (e.g. $\mathrm{Ca}^{2+}$ ) binding, and effector accessibility (e.g. kinases, phosphatases).

Otoferlin is thought to be involved in SV recycling and SV reformation processes in IHCs by interacting with phospholipids (see chapters 1.1.4 and 4.2.2) and proteins like AP-2 (Duncker et al, 2013; Jung et al, 2015a) and myosin VI (Heidrych et al, 2009; Roux et al, 2009; see also PLAs in Meese et al, 2017: Figure 3B and this thesis: chapter 3.3.10: Appendix Figure S1), or other yet-to-be-identified proteins (see chapter 1.1.4). These components are believed to form a large complex that facilitates pinching off of vesicles from large endosomal compartments and membrane invaginations (Duncker et al, 2013; Kononenko et al, 2014; Jung et al, 2015a; Strenzke et al, 2016; Chakrabarti et al, 2018; Pangrsic \& Vogl, 2018). Protein kinases control these processes in other cell types (see chapter 1.2) and in the case of otoferlin this thesis shows that CaMKII $\delta$ and PKC $\alpha$ possibly regulate both exocytic and endocytic processes near IHC's active zones to ensure fast and continuous SV recycling (Meese et al, 2017; this thesis: chapters 2 and 3$)$.

By affecting the folding of the protein, either locally (at the single domain level) or globally (leading in some cases to a complete collapse of the structure), OTOF mutations might hinder accessibility by kinases that regulate their function, like CaMKII and PKC, and/or by other interactors involved in membrane retrieval, SV recycling and SV reformation. This can ultimately lead to membrane turnover defects and enlarged SVs as it was observed in Otof

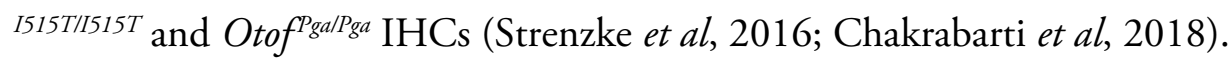




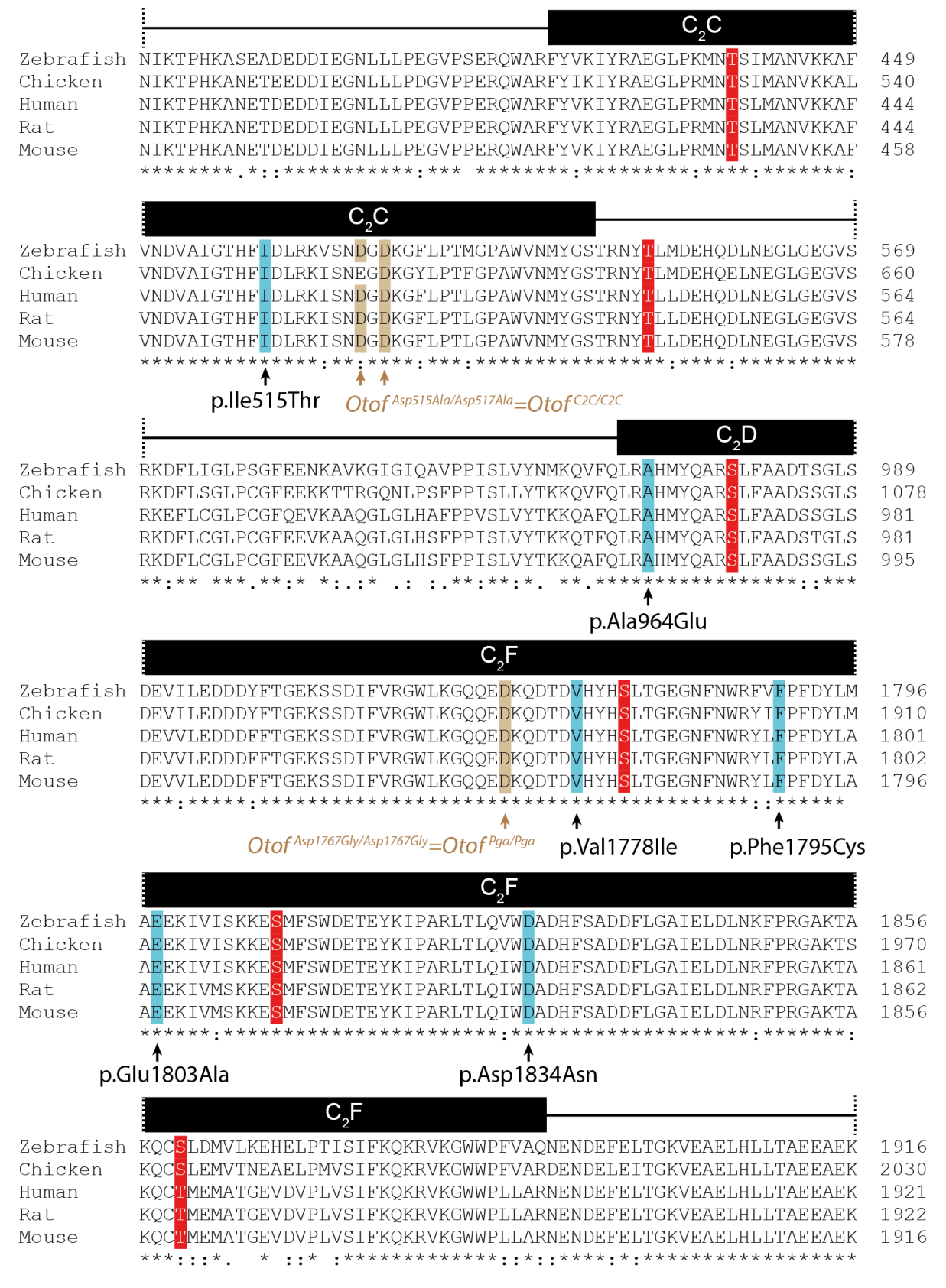

Figure 4.1. Position of known $O T O F$ mutations relative to CaMKII $\delta$ phosphorylation sites.

Most CaMKII $\delta$ phosphorylations sites (in red) are located in $\mathrm{C}_{2}$ domains, in conserved regions among different species. A few $O T O F$ mutations (in blue) are located within $\mathrm{C}_{2}$ domains and close to CaMKII $\delta$ 's phosphorylation hotspots. The Otof ${ }^{C 2 C / C 2 C}$ and Otof ${ }^{\text {Pgal Pga }}$ mutations (found only in mouse models) are labelled in brown. Sequence alignment was performed in CLUSTAL Omega (1.2.4), EMBL-EBI, using mouse (NP_001093865.1, variant 1), human (NP_919224.1), rat (NP_001263649.1), chicken (XP_015140684.1) and zebrafish (NP_001025283.1) otoferlin sequences. 
It is actually interesting to note that some OTOF/Otof mutations cluster near CaMKII $\delta$ and PKC $\alpha$ phosphorylation sites (Figures Figure 4.1. and Figure 4.2. ). Among others, the OTOF I515TIII5T (in the $\mathrm{C}_{2} \mathrm{C}$ domain) and the Otof ${ }^{\text {Pgal/ga }}$ (in the $\mathrm{C}_{2} \mathrm{~F}$ domain) mutations are located close to CaMKII $\delta$ phosphorylation hotspots, in regions conserved among species (Figure 4.1.). The p.Ile515Thr and Pga mutations affect not only the stability of otoferlin but also, in the case of the Pga mutation, its ability to bind $\mathrm{Ca}^{2+}$ (Pangrsic et al, 2010; Strenzke et al, 2016; Meese, 2015; Meese et al, 2017), ultimately leading to hearing impairment. Detailed implications of amino acid substitutions in p.Ile515Thr and Pga on protein folding and domain accessibility were already vastly discussed in chapter 4.2.2. $\mathrm{Ca}^{2+}$ binding was additionally shown to be regulated by phosphorylation (by CaMKIII) at least for two of the $\mathrm{C}_{2}$ domains, leading to an increased $\mathrm{Ca}^{2+}$ affinity of the $\mathrm{C}_{2} \mathrm{C}$ domain and decreased $\mathrm{Ca}^{2+}$ affinity of the $\mathrm{C}_{2} \mathrm{~F}$ domain upon phosphorylation (Meese et al, 2017). It is then clear that phosphorylation is an important regulatory event in the life of otoferlin, mediating $\mathrm{Ca}^{2+}$ binding and possibly also subcellular localization. Although $\mathrm{Ca}^{2+}$ binding was presumed to be also affected in the Otof ${ }^{C 2 C / C 2 C}$ mutant (Michalski et al, 2017), this mouse model was generated with the purpose of changing the $\mathrm{Ca}^{2+}$ binding ability of otoferlin by mutating the aspartate residues predicted to coordinate $\mathrm{Ca}^{2+}$. Therefore, any implications regarding $\mathrm{Ca}^{2+}$ binding changes due to close proximity to a CaMKII phosphorylation hotspot (Figure 4.1.) cannot be drawn.

The two OTOF pathogenic mutations p.Glu766del and p.Arg794His (Varga et al, 2006; Choi et al, 2009), located at the B and C helices of otoferlin's FerA domain, respectively, are located near the PKC $\alpha$ phosphorylation site S775 in otoferlin and were the only mutations found near PKC $\alpha$ 's phosphorylation sites (Figure 4.2. ). The p.Glu766del mutation was first described as prevalent in a Pakistani family, and is characterized by severe-to-profound hearing impairment (Choi et al, 2009). The p.Arg794His mutation was found in three individuals in one family from Iowa, USA, with all individuals heterozygous for the mutation and suffering from severeto-profound hearing impairment (Varga et al, 2006). As already mentioned, the phosphorylation at S775 in the FerA domain could possibly facilitate the interaction of FerA with membranes in a $\mathrm{Ca}^{2+}$-dependent manner (Harsini et al, 2018), by changing the folding and/or lipid-binding properties of the domain through conversion from a four-helix bundle to an inverted hydrophobic membrane-associating structure. If we consider the FerA domain to be an essential component for the membrane-associating properties of otoferlin, and absolutely necessary for its functions in SV recycling in IHCs, it is logic why these two mutations are pathogenic. The aforementioned mutations might on one hand change the overall folding and impede the access of the kinase or may affect the overall stability of the structure culminating in its collapse. The deletion of glutamine in p.Glu766del mutation, a naturally amphipathic amino acid with polar and hydrophobic areas, may render the FerA less hydrophobic affecting 
its interaction with or integration into membranes. For the p.Arg794His mutation, arginine, an amphipathic but positively charged amino acid, was replaced by a histidine, a more hydrophobic residue either considered uncharged or positively charged. Switching from an arginine to a histidine can turn helix $\mathrm{C}$ into a more hydrophobic surface that has higher affinity to negatively charged membrane surfaces. However, what could be seen as a gain-of-function mutation can be detrimental for otoferlin given its role in different processes, as otoferlin most probably needs to adopt different conformations and interact or not with membranes at different stages of the SV cycle. Conversely, conformational changes induced by phosphorylation and dephosphorylation processes are modifications that are more flexible when introduced in a protein.

A

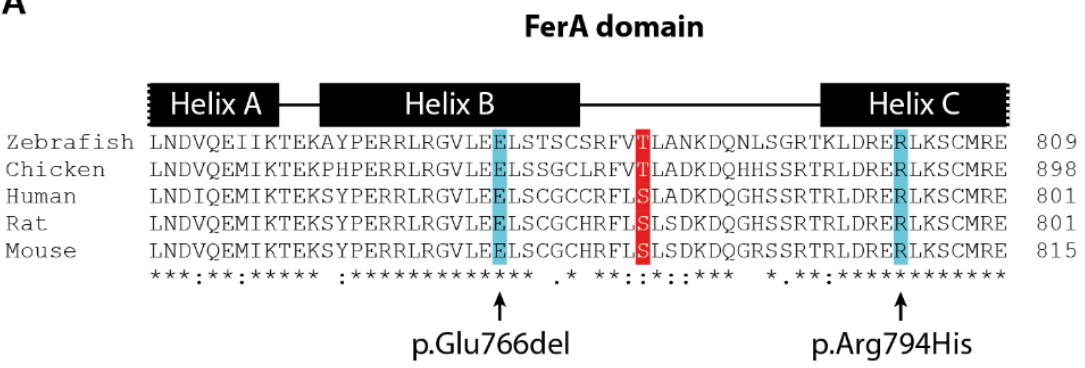

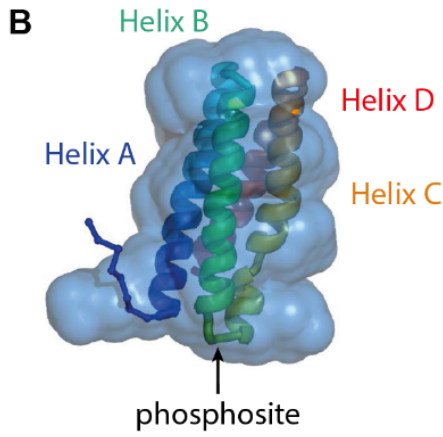

Figure 4.2. Position of p.Glu766del and p.Arg794His mutations relative to $\mathrm{PKC} \alpha$ phosphorylation site in FerA domain of otoferlin.

A. PKC $\alpha$ phosphorylation site (in red) in FerA domain of otoferlin, in a conserved region among different species $(S \rightarrow T$ variation in zebrafish and chicken). p.Glu766del and p.Arg794His mutations (in blue) are located within FerA helices B and C, respectively, and close to PKCa's phosphorylation site in this domain. Sequence alignment was performed in CLUSTAL Omega (1.2.4), EMBL-EBI, using mouse (NP_001093865.1, variant 1), human (NP_919224.1), rat (NP_001263649.1), chicken (XP_015140684.1) and zebrafish (NP_001025283.1) otoferlin sequences. FerA domain boundaries were determined by aligning otoferlin and dysferlin sequences, as done in Harsini et al, 2018. B. Position of the phosphorylation site in the modelled 3D structure of the FerA domain of dysferlin from Harsini et al, 2018.

\subsection{Outlook}

Sound perception, like the perception of light, demands sensitivity and dynamic range. Sound encoding between the sensory IHCs and the SGNs occurs with exceptionally high precision, reliability and dynamics over prolonged periods of stimulation. Ribbon synapses are highly specialized features that fulfill this task. With their tethering pool of SVs they assure fast exocytic rates and sustained replenishment of the pool of fusion-competent vesicles, allowing an indefatigable afferent transmission at high rates with sub-millisecond temporal precision (Sterling \& Matthews, 2005; Griesinger et al, 2005; Khimich et al, 2005). Auditory processing 
relies on $\mathrm{Ca}^{2+}$-triggered fast synchronous fusion of neurotransmitter-filled SVs with the IHC's active zone plasma membrane. Otoferlin is currently accepted as the main $\mathrm{Ca}^{2+}$ sensor for exocytosis, but it is also involved in synaptic vesicle reformation and recycling, endocytosis and active zone clearance (see chapter 1.1.4). It is known for some time that at least some modes of endocytosis in IHCs are $\mathrm{Ca}^{2+}$-dependent (Beutner et al, 2001). It is also known that depending on stimulus intensity, different modes of endocytosis may be induced, and though their $\mathrm{Ca}^{2+}$-dependency or $\mathrm{Ca}^{2+}$ sensor have not been confirmed, otoferlin has been proposed to fulfill this role at several steps of the SV cycle (Beutner et al, 2001; Neef et al, 2014; Revelo et al, 2014; Jung et al, 2015a; Strenzke et al, 2016; Michalski et al, 2017; Kroll et al, 2019). My data supports this notion, with otoferlin regulating endocytic processes in collaboration with other $\mathrm{Ca}^{2+}$-binding proteins (calbindin, parvalbumin and calretinin) rather than as a standalone protein.

In conventional synapses, protein kinases like CaMKII and PKC have been known to regulate exocytosis (by controlling protein interactions within the release apparatus) but also some forms of presynaptic plasticity (e.g. by controlling the refiling of the RRP), endocytosis and trafficking events (Südhof, 1995; Turner et al, 1999; Haberman et al, 2005; Jong et al, 2016). In this thesis I show that otoferlin's function is regulated by the combined action of CaMKII and PKC in an activity-dependent manner. Differential phosphorylation by these kinases might explain otoferlin's involvement in distinct steps of the SV cycle in IHCs. CaMKII phosphorylates otoferlin in an activity-dependent manner (i.e. upon strong IHC depolarization), thereby regulating $\mathrm{Ca}^{2+}$-binding properties of otoferlin, affecting the affinity to $\mathrm{Ca}^{2+}$ of at least some of its $\mathrm{C}_{2}$ domains (see Chapter 2 and Meese et al, 2017). Phosphorylation by PKC also occurred in an activity-dependent manner and was characterized by short-living accumulations of the kinase and otoferlin in common endocytic structures (Revelo et al, 2014; Strenzke et al, 2016). Otoferlin was also found to interact with calbindinD28k and myosin VI in an activity-dependent and PKC-dependent manner. My data suggest that PKC $\alpha$, otoferlin, and calbindin-D28k form a $\mathrm{Ca}^{2+}$-dependent complex involved in endocytic events. A comprehensive evaluation of capacitance measurements from calbindin knock-out IHCs (Pangršič et al, 2015) and actin polymerization studies (Vincent et al, 2015; Guillet et al, 2016; Tertrais et al, 2019) points toward a possible involvement of calbindin in ultrafast endocytosis in IHCs, but this theory needs further validation. Patch-clamp capacitance measurements with CaMKII and PKC activators and inhibitors will be essential to pinpoint the step(s) of the SV cycle these two kinases might be influencing: exocytosis, endocytosis or both. Immunoprecipitation assays with otoferlin (phosphomimetics, fulllength, deletion constructs, partial fragments, or a combination of all) could prove useful to assess the exact binding sites of otoferlin to CaMKII, PKC and calbindin-D28k. Otoferlin phosphomimetics of PKC $\alpha$ 's phosphorylation sites could help assessing the implications of these phosphorylations on otoferlin's function. For instance, liposome flotation assays with 
reconstituted wild-type or phosphomimetic otoferlin could help assessing a change in affinity towards lipidic membranes.

As it stands, these two studies are of crucial importance and constitute the first-time evidence for regulation of IHC's synaptic activity by protein kinases. The phosphorylation of otoferlin by CaMKII and PKC possibly establish a molecular switch triggering exocytic and/or endocytic events in IHCs. The requirement of a $\mathrm{Ca}^{2+}$ sensor for endocytosis can be then overcome by the combined action of several molecular players, as I propose here. 


\section{References}

Abiria SA \& Colbran RJ (2010) CaMKII associates with CaV1.2 L-type calcium channels via selected beta subunits to enhance regulatory phosphorylation. J. Neurochem. 112: 150-161

Ahmed S, Holt M, Riedel D \& Jahn R (2013) Small-scale isolation of synaptic vesicles from mammalian brain. Nature protocols 8: 998-1009

Airaksinen L, Virkkala J, Aarnisalo A, Meyer M, Ylikoski J \& Airaksinen MS (2000) Lack of calbindinD28k does not affect hearing level or survival of hair cells in acoustic trauma. ORL J. Otorhinolaryngol. Relat. Spec. 62: 9-12

Al-Moyed H (2019) Dual-AAV mediated transfer of full-length otoferlin cDNA into auditory inner hair cells and the effects of different mutations in the OTOF gene on the protein levels and cellular distribution of otoferlin in auditory inner hair cells. Available at: https://ediss.unigoettingen.de/handle/11858/00-1735-0000-002E-E626-E [Accessed August 19, 2019]

Al-Moyed H, Cepeda AP, Jung S, Moser T, Kügler S \& Reisinger E (2019) A dual-AAV approach restores fast exocytosis and partially rescues auditory function in deaf otoferlin knock-out mice. EMBO Mol Med 11: e9396

Alpadi K, Magupalli VG, Käppel S, Köblitz L, Schwarz K, Seigel GM, Sung C-H \& Schmitz F (2008) RIBEYE Recruits Munc119, a Mammalian Ortholog of the Caenorhabditis elegans Protein unc119, to Synaptic Ribbons of Photoreceptor Synapses. J. Biol. Chem. 283: 26461-26467

Altrock WD, tom Dieck S, Sokolov M, Meyer AC, Sigler A, Brakebusch C, Fässler R, Richter K, Boeckers TM, Potschka H, Brandt C, Löscher W, Grimberg D, Dresbach T, Hempelmann A, Hassan H, Balschun D, Frey JU, Brandstätter JH, Garner CC, et al (2003) Functional inactivation of a fraction of excitatory synapses in mice deficient for the active zone protein bassoon. Neuron 37: $787-800$

Alvi F, Idkowiak-Baldys J, Baldys A, Raymond JR \& Hannun YA (2007) Regulation of membrane trafficking and endocytosis by protein kinase $\mathrm{C}$ : emerging role of the pericentrion, a novel protein kinase C-dependent subset of recycling endosomes. Cell. Mol. Life Sci. 64: 263-270

Ando S, Tokui T, Yamauchi T, Sugiura H, Tanabe K \& Inagaki M (1991) Evidence that Ser-82 is a unique phosphorylation site on vimentin for $\mathrm{Ca} 2(+)$-calmodulin-dependent protein kinase II. Biochem. Biophys. Res. Commun. 175: 955-962

Antal CE, Callender JA, Kornev AP, Taylor SS \& Newton AC (2015) Intramolecular C2 DomainMediated Autoinhibition of Protein Kinase C $\beta$ II. Cell Rep 12: 1252-1260

Antal CE, Violin JD, Kunkel MT, Skovsø S \& Newton AC (2014) Intramolecular conformational changes optimize protein kinase C signaling. Chem. Biol. 21: 459-469

Atanassov I \& Urlaub H (2013) Increased proteome coverage by combining PAGE and peptide isoelectric focusing: comparative study of gel-based separation approaches. Proteomics 13: 2947-2955 
Augustin I, Rosenmund C, Südhof TC \& Brose N (1999) Munc13-1 is essential for fusion competence of glutamatergic synaptic vesicles. Nature 400: 457-461

Bai J, Hu Z, Dittman JS, Pym ECG \& Kaplan JM (2010) Endophilin Functions as a Membrane-Bending Molecule and Is Delivered to Endocytic Zones by Exocytosis. Cell 143: 430-441

Barclay JW, Craig TJ, Fisher RJ, Ciufo LF, Evans GJO, Morgan A \& Burgoyne RD (2003) Phosphorylation of Munc18 by protein kinase $\mathrm{C}$ regulates the kinetics of exocytosis. Journal of Biological Chemistry 278: 10538-10545

Becker KP \& Hannun YA (2003) cPKC-dependent sequestration of membrane-recycling components in a subset of recycling endosomes. J. Biol. Chem. 278: 52747-52754

Becker L, Schnee ME, Niwa M, Sun W, Maxeiner S, Talaei S, Kachar B, Rutherford MA \& Ricci AJ (2018) The presynaptic ribbon maintains vesicle populations at the hair cell afferent fiber synapse. eLife Sciences 7: e30241

Bellocchio EE, Reimer RJ, Fremeau RT Jr \& Edwards RH (2000) Uptake of glutamate into synaptic vesicles by an inorganic phosphate transporter. Science 289: 957-960

Bennett MK, Calakos N \& Scheller RH (1992) Syntaxin: a synaptic protein implicated in docking of synaptic vesicles at presynaptic active zones. Science 257: 255-259

Berggard T (2002) Calbindin D28k Exhibits Properties Characteristic of a Ca2+ Sensor. Journal of Biological Chemistry 277: 16662-16672

Beurg M, Michalski N, Safieddine S, Bouleau Y, Schneggenburger R, Chapman ER, Petit C \& Dulon D (2010) Control of exocytosis by synaptotagmins and otoferlin in auditory hair cells. J. Neurosci 30: $13281-13290$

Beutner D \& Moser T (2001) The presynaptic function of mouse cochlear inner hair cells during development of hearing. J Neurosci 21: 4593

Beutner D, Voets T, Neher E \& Moser T (2001) Calcium dependence of exocytosis and endocytosis at the cochlear inner hair cell afferent synapse. Neuron 29: 681-690

Blom N, Sicheritz-Pontén T, Gupta R, Gammeltoft S \& Brunak S (2004) Prediction of post-translational glycosylation and phosphorylation of proteins from the amino acid sequence. Proteomics 4: 16331649

van den Bogaart G, Meyenberg K, Diederichsen U \& Jahn R (2012) Phosphatidylinositol 4,5-bisphosphate increases the Ca2+ affinity of synaptotagmin-1 40-fold. Journal of Biological Chemistry Available at: http://www.jbc.org/cgi/doi/10.1074/jbc.M112.343418 [Accessed March 30, 2012]

Boucrot E, Saffarian S, Zhang R \& Kirchhausen T (2010) Roles of AP-2 in clathrin-mediated endocytosis. PLoS ONE 5: e10597

Brager DH, Cai X \& Thompson SM (2003) Activity-dependent activation of presynaptic protein kinase C mediates post-tetanic potentiation. Nat. Neurosci. 6: 551-552

Brandt A, Khimich D \& Moser T (2005) Few CaV1. 3 channels regulate the exocytosis of a synaptic vesicle at the hair cell ribbon synapse. J Neurosci 25: 11577 
Brandt A, Striessnig J \& Moser T (2003) CaV1. 3 channels are essential for development and presynaptic activity of cochlear inner hair cells. J Neurosci 23: 10832-10840

Brocke L, Srinivasan M \& Schulman H (1995) Developmental and regional expression of multifunctional Ca2+/calmodulin-dependent protein kinase isoforms in rat brain. J. Neurosci. 15: 6797-6808

Brose N, Hofmann K, Hata Y \& Südhof TC (1995) Mammalian Homologues of Caenorhabditis elegans unc-13 Gene Define Novel Family of C-domain Proteins. Journal of Biological Chemistry 270: 25273

Brose N, Petrenko AG, Südhof TC \& Jahn R (1992) Synaptotagmin: a calcium sensor on the synaptic vesicle surface. Science 256: 1021-1025

Burgin KE, Waxham MN, Rickling S, Westgate SA, Mobley WC \& Kelly PT (1990) In situ hybridization histochemistry of $\mathrm{Ca} 2+/$ calmodulin-dependent protein kinase in developing rat brain. J. Neurosci. 10: $1788-1798$

Callender JA \& Newton AC (2017) Conventional protein kinase C in the brain: 40 years later. Neuronal Signaling 1: NS20160005

Cao H, Garcia F \& McNiven MA (1998) Differential distribution of dynamin isoforms in mammalian cells. Molecular biology of the cell 9: 2595

Capogna M, Gähwiler BH \& Thompson SM (1995) Presynaptic enhancement of inhibitory synaptic transmission by protein kinases $\mathrm{A}$ and $\mathrm{C}$ in the rat hippocampus in vitro. The Journal of neuroscience: the official journal of the Society for Neuroscience 15: 1249-60

Cases-Langhoff C, Voss B, Garner AM, Appeltauer U, Takei K, Kindler S, Veh RW, De Camilli P, Gundelfinger ED \& Garner CC (1996) Piccolo, a novel $420 \mathrm{kDa}$ protein associated with the presynaptic cytomatrix. Eur. J. Cell Biol. 69: 214-223

Castagna M, Takai Y, Kaibuchi K, Sano K, Kikkawa U \& Nishizuka Y (1982) Direct activation of calciumactivated, phospholipid-dependent protein kinase by tumor-promoting phorbol esters. J. Biol. Chem. 257: 7847-7851

Catterall WA (2011) Voltage-gated calcium channels. Cold Spring Harb Perspect Biol 3: a003947

Catterall WA \& Few AP (2008) Calcium channel regulation and presynaptic plasticity. Neuron 59: 882901

Cepeda AP, Al-Moyed H, Lenz C, Urlaub H \& Reisinger E (2019) PKC $\alpha$-dependent interaction of otoferlin and calbindin: evidence for regulation of endocytosis in inner hair cells Neuroscience Available at: http://biorxiv.org/lookup/doi/10.1101/779520

Cesca F, Baldelli P, Valtorta F \& Benfenati F (2010) The synapsins: Key actors of synapse function and plasticity. Progress in Neurobiology 91: 313-348

Chakrabarti R, Michanski S \& Wichmann C (2018) Vesicle sub-pool organization at inner hair cell ribbon synapses. EMBO reports: e44937 
Chao LH, Stratton MM, Lee I-H, Rosenberg OS, Levitz J, Mandell DJ, Kortemme T, Groves JT, Schulman H \& Kuriyan J (2011) A mechanism for tunable autoinhibition in the structure of a human Ca2+/calmodulin- dependent kinase II holoenzyme. Cell 146: 732-745

Chapochnikov NM, Takago H, Huang C-H, Pangršič T, Khimich D, Neef J, Auge E, Göttfert F, Hell SW, Wichmann C, Wolf F \& Moser T (2014) Uniquantal Release through a Dynamic Fusion Pore Is a Candidate Mechanism of Hair Cell Exocytosis. Neuron 17: 1389-1403

Cho W \& Stahelin RV (2006) Membrane binding and subcellular targeting of C2 domains. Biochim. Biophys. Acta 1761: 838-849

Choi BY, Ahmed ZM, Riazuddin S, Bhinder MA, Shahzad M, Husnain T, Riazuddin S, Griffith AJ \& Friedman TB (2009) Identities and frequencies of mutations of the otoferlin gene (OTOF) causing DFNB9 deafness in Pakistan. Clinical Genetics 75: 237-243

Cijsouw T, Weber JP, Broeke JH, Broek JAC, Schut D, Kroon T, Saarloos I, Verhage M \& Toonen RF (2014) Munc18-1 redistributes in nerve terminals in an activity- and PKC-dependent manner. Journal of Cell Biology 204: 759-775

Codazzi F, Teruel MN \& Meyer T (2001) Control of astrocyte $\mathrm{Ca}(2+)$ oscillations and waves by oscillating translocation and activation of protein kinase C. Curr. Biol. 11: 1089-1097

Cordey M \& Pike CJ (2006) Conventional protein kinase C isoforms mediate neuroprotection induced by phorbol ester and estrogen. J. Neurochem. 96: 204-217

Costes SV, Daelemans D, Cho EH, Dobbin Z, Pavlakis G \& Lockett S (2004) Automatic and quantitative measurement of protein-protein colocalization in live cells. Biophysical journal 86: 3993-4003

Cousin MA \& Robinson PJ (2001) The dephosphins: dephosphorylation by calcineurin triggers synaptic vesicle endocytosis. Trends in neurosciences 24: 659-665

Cui G, Meyer AC, Calin-Jageman I, Neef J, Haeseleer F, Moser T \& Lee A (2007) Ca2+-binding proteins tune Ca2+-feedback to Cav1. 3 channels in mouse auditory hair cells. The Journal of Physiology 585: 791-803

Deguchi-Tawarada M, Inoue E, Takao-Rikitsu E, Inoue M, Kitajima I, Ohtsuka T \& Takai Y (2006) Active zone protein CAST is a component of conventional and ribbon synapses in mouse retina. $J$. Comp. Neurol 495: 480-496

Denker A \& Rizzoli SO (2010) Synaptic Vesicle Pools: An Update. Front Synaptic Neurosci 2:

Dick O, Hack I, Altrock WD, Garner CC, Gundelfinger ED \& Brandstätter JH (2001) Localization of the presynaptic cytomatrix protein Piccolo at ribbon and conventional synapses in the rat retina: Comparison with Bassoon. The Journal of Comparative Neurology 439: 224-234

tom Dieck S, Altrock WD, Kessels MM, Qualmann B, Regus H, Brauner D, Fejtová A, Bracko O, Gundelfinger ED \& Brandstätter JH (2005) Molecular dissection of the photoreceptor ribbon synapse: physical interaction of Bassoon and RIBEYE is essential for the assembly of the ribbon complex. J Cell Biol 168: 825-836 
tom Dieck S, Sanmartí-Vila L, Langnaese K, Richter K, Kindler S, Soyke A, Wex H, Smalla KH, Kämpf U, Fränzer JT, Stumm M, Garner CC \& Gundelfinger ED (1998) Bassoon, a novel zinc-finger CAG/glutamine-repeat protein selectively localized at the active zone of presynaptic nerve terminals. J Cell Biol 142: 499-509

Dou H, Vazquez AE, Namkung Y, Chu H, Cardell EL, Nie L, Parson S, Shin H-S \& Yamoah EN (2004) Null mutation of alpha1D Ca2+ channel gene results in deafness but no vestibular defect in mice. $J$ Assoc Res Otolaryngol 5: 215-226

Dulon D, Safieddine S, Jones SM \& Petit C (2009) Otoferlin Is Critical for a Highly Sensitive and Linear Calcium-Dependent Exocytosis at Vestibular Hair Cell Ribbon Synapses. The Journal of Neuroscience 29: 10474-10487

Duncker SV, Franz C, Kuhn S, Schulte U, Campanelli D, Brandt N, Hirt B, Fakler B, Blin N, Ruth P, Engel J, Marcotti W, Zimmermann U \& Knipper M (2013) Otoferlin Couples to ClathrinMediated Endocytosis in Mature Cochlear Inner Hair Cells. J. Neurosci. 33: 9508-9519

Edmonds BW (2004) Evidence that fast exocytosis can be predominantly mediated by vesicles not docked at active zones in frog saccular hair cells. J Physiol 560: 439-450

Erondu NE \& Kennedy MB (1985) Regional distribution of type II Ca2+/calmodulin-dependent protein kinase in rat brain. J. Neurosci. 5: 3270-3277

Evans JH, Murray D, Leslie CC \& Falke JJ (2006) Specific translocation of protein kinase Calpha to the plasma membrane requires both Ca2+ and PIP2 recognition by its C2 domain. Mol. Biol. Cell 17: $56-66$

Feng X, Becker KP, Stribling SD, Peters KG \& Hannun YA (2000) Regulation of receptor-mediated protein kinase $\mathrm{C}$ membrane trafficking by autophosphorylation. J. Biol. Chem. 275: 17024-17034

Feng X, Zhang J, Barak LS, Meyer T, Caron MG \& Hannun YA (1998) Visualization of dynamic trafficking of a protein kinase $\mathrm{C}$ betaII/green fluorescent protein conjugate reveals differences in $\mathrm{G}$ protein-coupled receptor activation and desensitization. J. Biol. Chem. 273: 10755-10762

Ferguson SM, Brasnjo G, Hayashi M, Wolfel M, Collesi C, Giovedi S, Raimondi A, Gong L-W, Ariel P, Paradise S, O’Toole E, Flavell R, Cremona O, Miesenbock G, Ryan TA \& De Camilli P (2007) A Selective Activity-Dependent Requirement for Dynamin 1 in Synaptic Vesicle Endocytosis. Science 316: $570-574$

Ferguson SM \& De Camilli P (2012) Dynamin, a membrane-remodelling GTPase. Nature Reviews Molecular Cell Biology 13: 75-88

Fernandez I, Araç D, Ubach J, Gerber SH, Shin O, Gao Y, Anderson RG, Südhof TC \& Rizo J (2001) Three-dimensional structure of the synaptotagmin 1 C2B-domain: synaptotagmin 1 as a phospholipid binding machine. Neuron 32: 1057-1069

Fettiplace R (2017) Hair Cell Transduction, Tuning, and Synaptic Transmission in the Mammalian Cochlea. Compr Physiol 7: 1197-1227

Fettiplace R \& Kim KX (2014) The physiology of mechanoelectrical transduction channels in hearing. Physiol. Rev. 94: 951-986 
Frank T, Rutherford MA, Strenzke N, Neef A, Pangršič T, Khimich D, Fejtova A, Gundelfinger ED, Liberman MC, Harke B, Bryan KE, Lee A, Egner A, Riedel D \& Moser T (2010) Bassoon and the synaptic ribbon organize $\mathrm{Ca}^{2}+$ channels and vesicles to add release sites and promote refilling. Neuron 68: 724-738

Fremeau RT, Troyer MD, Pahner I, Nygaard GO, Tran CH, Reimer RJ, Bellocchio EE, Fortin D, StormMathisen J \& Edwards RH (2001) The Expression of Vesicular Glutamate Transporters Defines Two Classes of Excitatory Synapse. Neuron 31: 247-260

Frolenkov GI, Belyantseva IA, Friedman TB \& Griffith AJ (2004) Genetic insights into the morphogenesis of inner ear hair cells. Nat Rev Genet 5: 489-498

Fuchs PA (2005) Time and intensity coding at the hair cell's ribbon synapse. The Journal of Physiology 566: $7-12$

Fuchs PA, Glowatzki E \& Moser T (2003) The afferent synapse of cochlear hair cells. Current Opinion in Neurobiology 13: 452-458

Fukunaga K, Muller D \& Miyamoto E (1995) Increased phosphorylation of Ca2+/calmodulin-dependent protein kinase II and its endogenous substrates in the induction of long-term potentiation. J. Biol. Chem. 270: 6119-6124

Gaertner TR, Kolodziej SJ, Wang D, Kobayashi R, Koomen JM, Stoops JK \& Waxham MN (2004) Comparative analyses of the three-dimensional structures and enzymatic properties of alpha, beta, gamma and delta isoforms of Ca2+-calmodulin-dependent protein kinase II. J. Biol. Chem. 279: 12484-12494

Genç Ö, Kochubey O, Toonen RF, Verhage M \& Schneggenburger R (2014) Munc18-1 is a dynamically regulated PKC target during short-term enhancement of transmitter release. eLife 2014: 1-19

Genoud S, Pralong W, Riederer BM, Eder L, Catsicas S \& Muller D (2001) Activity-Dependent Phosphorylation of SNAP-25 in Hippocampal Organotypic Cultures. Journal of Neurochemistry 72: 1699-1706

Geppert M, Archer BT 3rd \& Südhof TC (1991) Synaptotagmin II. A novel differentially distributed form of synaptotagmin. J. Biol. Chem. 266: 13548-13552

Geppert M, Goda Y, Hammer RE, Li C, Rosahl TW, Stevens CF \& Südhof TC (1994) Synaptotagmin I: A major Ca2+ sensor for transmitter release at a central synapse. Cell 79: 717-727

Geppert M, Goda Y, Stevens CF \& Südhof TC (1997) The small GTP-binding protein Rab3A regulates a late step in synaptic vesicle fusion. Nature 387: 810-814

Gijón MA, Spencer DM, Kaiser AL \& Leslie CC (1999) Role of phosphorylation sites and the C2 domain in regulation of cytosolic phospholipase A2. J. Cell Biol. 145: 1219-1232

Giraudo CG, Eng WS, Melia TJ \& Rothman JE (2006) A clamping mechanism involved in SNAREdependent exocytosis. Science 313: 676-680

Glowatzki E \& Fuchs PA (2002) Transmitter release at the hair cell ribbon synapse. Nat Neurosci 5: 147154 
González MI, Bannerman PG \& Robinson MB (2003) Phorbol myristate acetate-dependent interaction of protein kinase Calpha and the neuronal glutamate transporter EAAC1. J. Neurosci. 23: 5589-5593

Goodyear RJ, Legan PK, Christiansen JR, Xia B, Korchagina J, Gale JE, Warchol ME, Corwin JT \& Richardson GP (2010) Identification of the hair cell soma-1 antigen, HCS-1, as otoferlin. J. Assoc. Res. Otolaryngol. 11: 573-586

Gould CM, Antal CE, Reyes G, Kunkel MT, Adams RA, Ziyar A, Riveros T \& Newton AC (2011) Active site inhibitors protect protein kinase $\mathrm{C}$ from dephosphorylation and stabilize its mature form. $J$. Biol. Chem. 286: 28922-28930

Grabner CP \& Moser T (2018) Individual synaptic vesicles mediate stimulated exocytosis from cochlear inner hair cells. PNAS 115: 12811-12816

Graydon CW, Cho S, Li G-L, Kachar B \& von Gersdorff H (2011) Sharp Ca2+ Nanodomains beneath the Ribbon Promote Highly Synchronous Multivesicular Release at Hair Cell Synapses. J. Neurosci. 31: $16637-16650$

Graydon CW, Zhang J, Oesch NW, Sousa AA, Leapman RD \& Diamond JS (2014) Passive Diffusion as a Mechanism Underlying Ribbon Synapse Vesicle Release and Resupply. J. Neurosci. 34: 89488962

Greengard P, Valtorta F, Czernik AJ \& Benfenati F (1993) Synaptic vesicle phosphoproteins and regulation of synaptic function. Science 259: 780-785

Gregory FD, Bryan KE, Pangrsic T, Calin-Jageman IE, Moser T \& Lee A (2011) Harmonin inhibits presynaptic Cav1.3 Ca2+ channels in mouse inner hair cells. Nat Neurosci 14: 1109-1111

Griesinger CB, Richards CD \& Ashmore JF (2005) Fast vesicle replenishment allows indefatigable signalling at the first auditory synapse. Nature 435: 212-215

Guerrero-Valero M, Ferrer-Orta C, Querol-Audí J, Marin-Vicente C, Fita I, Gómez-Fernández JC, Verdaguer N \& Corbalán-García S (2009) Structural and mechanistic insights into the association of PKCalpha-C2 domain to PtdIns(4,5)P2. Proc. Natl. Acad. Sci. U.S.A. 106: 6603-6607

Guillet M, Sendin G, Bourien J, Puel J-L \& Nouvian R (2016) Actin Filaments Regulate Exocytosis at the Hair Cell Ribbon Synapse. J. Neurosci. 36: 649-654

Haberman Y, Ziv I, Gorzalczany Y, Fukuda M \& Sagi-Eisenberg R (2005) Classical protein kinase C(s) regulates targeting of synaptotagmin IX to the endocytic recycling compartment. J. Cell. Sci. 118: $1641-1649$

Hallermann S, Fejtova A, Schmidt H, Weyhersmüller A, Silver RA, Gundelfinger ED \& Eilers J (2010) Bassoon Speeds Vesicle Reloading at a Central Excitatory Synapse. Neuron 68: 710-723

Hams N, Padmanarayana M, Qiu W \& Johnson CP (2017) Otoferlin is a multivalent calcium-sensitive scaffold linking SNAREs and calcium channels. Proc. Natl. Acad. Sci. U.S.A. 114: 8023-8028

Harris KM \& Sultan P (1995) Variation in the number, location and size of synaptic vesicles provides an anatomical basis for the nonuniform probability of release at hippocampal CA1 synapses. Neuropharmacology 34: 1387-1395 
Harsini FM, Chebrolu S, Fuson KL, White MA, Rice AM \& Sutton RB (2018) FerA is a MembraneAssociating Four-Helix Bundle Domain in the Ferlin Family of Membrane-Fusion Proteins. Sci Rep 8: 10949

Hata Y, Slaughter CA \& Südhof TC (1993) Synaptic vesicle fusion complex contains unc-18 homologue bound to syntaxin. Nature $366: 347-351$

Heffner HE \& Heffner RS (2007) Hearing ranges of laboratory animals. J. Am. Assoc. Lab. Anim. Sci. 46: $20-22$

Heidrych P, Zimmermann U, Breß A, Pusch CM, Ruth P, Pfister M, Knipper M \& Blin N (2008) Rab8b GTPase, a protein transport regulator, is an interacting partner of otoferlin, defective in a human autosomal recessive deafness form. Hum. Mol. Genet. 17: 3814-3821

Heidrych P, Zimmermann U, Kuhn S, Franz C, Engel J, Duncker SV, Hirt B, Pusch CM, Ruth P, Pfister M, Marcotti W, Blin N \& Knipper M (2009) Otoferlin interacts with myosin VI: implications for maintenance of the basolateral synaptic structure of the inner hair cell. Hum. Mol. Genet. 18: 27792790

Helfmann S, Neumann P, Tittmann K, Moser T, Ficner R \& Reisinger E (2011) The crystal structure of the $\mathrm{C}_{2} \mathrm{~A}$ domain of otoferlin reveals an unconventional top loop region. J. Mol. Biol 406: 479-490

Hell JW \& Jahn R (2006) Preparation of Synaptic Vesicles from Mammalian Brain. In Cell Biology pp 8590. Elsevier

Henry L \& Sheff DR (2008) Rab8 regulates basolateral secretory, but not recycling, traffic at the recycling endosome. Mol. Biol. Cell 19: 2059-2068

Hermelin B, Cherqui G, Bertrand F, Wicek D, Paul A, Garcia I \& Picard J (1988) Phorbol ester-induced protein kinase $\mathrm{C}$ translocation and lysosomal enzyme release in normal and cystic fibrosis fibroblasts. FEBS Lett. 229: 161-166

Hilfiker S \& Augustine GJ (1999) Regulation of synaptic vesicle fusion by protein kinase C. Journal of Physiology 515: 1

Hirling H \& Scheller RH (1996) Phosphorylation of synaptic vesicle proteins: modulation of the alpha SNAP interaction with the core complex. PNAS 93: 11945-11949

Hori T, Takai Y \& Takahashi T (1999) Presynaptic mechanism for phorbol ester-induced synaptic potentiation. J. Neurosci. 19: 7262-7267

Hu Y, Qu L \& Schikorski T (2008) Mean synaptic vesicle size varies among individual excitatory hippocampal synapses. Synapse 62: 953-957

Huang C-H \& Moser T (2018) Ca2+ Regulates the Kinetics of Synaptic Vesicle Fusion at the Afferent Inner Hair Cell Synapse. Front Cell Neurosci 12: 364

Huang H-D, Lee T-Y, Tzeng S-W \& Horng J-T (2005) KinasePhos: a web tool for identifying protein kinase-specific phosphorylation sites. Nucleic Acids Res. 33: W226-229

Huang XP, Pi Y, Lokuta AJ, Greaser ML \& Walker JW (1997) Arachidonic acid stimulates protein kinase C-epsilon redistribution in heart cells. J. Cell. Sci. 110 ( Pt 14): 1625-1634 
Hudmon A \& Schulman H (2002) Neuronal CA2+/calmodulin-dependent protein kinase II: the role of structure and autoregulation in cellular function. Annu. Rev. Biochem. 71: 473-510

Hudspeth AJ (1997) How Hearing Happens. Neuron 19: 947-950

Hunter T (2007) The age of crosstalk: phosphorylation, ubiquitination, and beyond. Mol. Cell 28: 730738

Huttner WB, Schiebler W, Greengard P \& De Camilli P (1983) Synapsin I (protein I), a nerve terminalspecific phosphoprotein. III. Its association with synaptic vesicles studied in a highly purified synaptic vesicle preparation. The Journal of cell biology 96: 1374-88

Inoue M, Kishimoto A, Takai Y \& Nishizuka Y (1977) Studies on a cyclic nucleotide-independent protein kinase and its proenzyme in mammalian tissues. II. Proenzyme and its activation by calciumdependent protease from rat brain. J. Biol. Chem. 252: 7610-7616

Jackman SL, Choi S-Y, Thoreson WB, Rabl K, Bartoletti TM \& Kramer RH (2009) Role of the synaptic ribbon in transmitting the cone light response. Nat Neurosci 12: 303

Jahn R \& Scheller RH (2006) SNAREs--engines for membrane fusion. Nat. Rev. Mol. Cell Biol. 7: 631643

Jean P, Lopez de la Morena D, Michanski S, Jaime Tobón LM, Chakrabarti R, Picher MM, Neef J, Jung S, Gültas M, Maxeiner S, Neef A, Wichmann C, Strenzke N, Grabner C \& Moser T (2018) The synaptic ribbon is critical for sound encoding at high rates and with temporal precision. Elife 7:

Jenkins MA, Christel CJ, Jiao Y, Abiria S, Kim KY, Usachev YM, Obermair GJ, Colbran RJ \& Lee A (2010) $\mathrm{Ca} 2+-$ dependent facilitation of Cav1.3 Ca2+ channels by densin and $\mathrm{Ca} 2+/$ calmodulindependent protein kinase II. J. Neurosci. 30: 5125-5135

Jiménez JL \& Bashir R (2007) In silico functional and structural characterisation of ferlin proteins by mapping disease-causing mutations and evolutionary information onto three-dimensional models of their C2 domains. J. Neurol. Sci 260: 114-123

Jing Z, Rutherford MA, Takago H, Frank T, Fejtova A, Khimich D, Moser T \& Strenzke N (2013) Disruption of the presynaptic cytomatrix protein bassoon degrades ribbon anchorage, multiquantal release, and sound encoding at the hair cell afferent synapse. J Neurosci 33: 4456-4467

Jockusch WJ, Speidel D, Sigler A, Sørensen JB, Varoqueaux F, Rhee J-S \& Brose N (2007) CAPS-1 and CAPS-2 Are Essential Synaptic Vesicle Priming Proteins. Cell 131: 796-808

Johnson CP (2017) Emerging Functional Differences between the Synaptotagmin and Ferlin Calcium Sensor Families. Biochemistry 56: 6413-6417

Johnson CP \& Chapman ER (2010) Otoferlin is a calcium sensor that directly regulates SNARE-mediated membrane fusion. J. Cell Biol 191: 187-197

Johnson SL, Franz C, Kuhn S, Furness DN, Ruttiger L, Munkner S, Rivolta MN, Seward EP, Herschman HR, Engel J, Knipper M \& Marcotti W (2010) Synaptotagmin IV determines the linear Ca2+ dependence of vesicle fusion at auditory ribbon synapses. Nat Neurosci 13: 45-52 
Johnson SL, Olt J, Cho S, von Gersdorff H \& Marcotti W (2017) The Coupling between Ca2+ Channels and the Exocytotic Ca2+ Sensor at Hair Cell Ribbon Synapses Varies Tonotopically along the Mature Cochlea. J. Neurosci. 37: 2471-2484

Jong APH de, Meijer M, Saarloos I, Cornelisse LN, Toonen RFG, Sørensen JB \& Verhage M (2016) Phosphorylation of synaptotagmin-1 controls a post-priming step in PKC-dependent presynaptic plasticity. Proceedings of the National Academy of Sciences 113: 5095-5100

Jung S, Maritzen T, Wichmann C, Jing Z, Neef A, Revelo NH, Al-Moyed H, Meese S, Wojcik SM, Panou I, Bulut H, Schu P, Ficner R, Reisinger E, Rizzoli SO, Neef J, Strenzke N, Haucke V \& Moser T (2015a) Disruption of adaptor protein $2 \mu(\mathrm{AP}-2 \mu)$ in cochlear hair cells impairs vesicle reloading of synaptic release sites and hearing. EMBO J. 34: 2686-2702

Jung S, Oshima-Takago T, Chakrabarti R, Wong AB, Jing Z, Yamanbaeva G, Picher MM, Wojcik SM, Göttfert F, Predoehl F, Michel K, Hell SW, Schoch S, Strenzke N, Wichmann C \& Moser T (2015b) Rab3-interacting molecules $2 \alpha$ and $2 \beta$ promote the abundance of voltage-gated CaV1.3 Ca2+ channels at hair cell active zones. Proc. Natl. Acad. Sci. U.S.A. 112: E3141-E3149

Kalbfleisch T, Cambon A \& Wattenberg BW (2007) A Bioinformatics Approach to Identifying TailAnchored Proteins in the Human Genome. Traffic 8: 1687-1694

Kamin D, Revelo NH \& Rizzoli SO (2014) FM Dye Photo-Oxidation as a Tool for Monitoring Membrane Recycling in Inner Hair Cells. PLoS ONE 9: e88353

Kandel ER, Schwartz JH \& Jessell T (2012) Principles of neural science New York: McGraw-Hill Medical Available at: http://www.myilibrary.com?id=396874 [Accessed April 22, 2016]

Kang CH, Moon BC, Park HC, Koo SC, Chi YH, Cheong YH, Yoon B-D, Lee SY \& Kim CY (2013) Rice Small C2-Domain Proteins Are Phosphorylated by Calcium-Dependent Protein Kinase. Mol Cells 35: 381-387

Kantardzhieva A, Liberman MC \& Sewell WF (2013) Quantitative analysis of ribbons, vesicles, and cisterns at the cat inner hair cell synapse: Correlations with spontaneous rate. Journal of Comparative Neurology 521: 3260-3271

Kataoka M, Kuwahara R, Iwasaki S, Shoji-Kasai Y \& Takahashi M (2000) Nerve Growth Factor-Induced Phosphorylation of SNAP-25 in PC12 Cells. Journal of Neurochemistry 74: 2058-2066

Keen JH (1987) Clathrin assembly proteins: affinity purification and a model for coat assembly. J. Cell Biol. 105: $1989-1998$

Khimich D, Nouvian R, Pujol R, tom Dieck S, Egner A, Gundelfinger ED \& Moser T (2005) Hair cell synaptic ribbons are essential for synchronous auditory signalling. Nature 434: 889-894

Kiang NYS (2011) Peripheral Neural Processing of Auditory Information. In Comprehensive Physiology pp 639-674. Available at: https://onlinelibrary.wiley.com/doi/10.1002/cphy.cp010315 [Accessed June 28, 2019]

Kim JH, Lee S-R, Li L-H, Park H-J, Park J-H, Lee KY, Kim M-K, Shin BA \& Choi S-Y (2011) High Cleavage Efficiency of a 2A Peptide Derived from Porcine Teschovirus-1 in Human Cell Lines, Zebrafish and Mice. PLoS One 6: e18556 
Kirchhausen T, Nathanson KL, Matsui W, Vaisberg A, Chow EP, Burne C, Keen JH \& Davis AE (1989) Structural and functional division into two domains of the large (100- to $115-\mathrm{kDa})$ chains of the clathrin-associated protein complex AP-2. Proc Natl Acad Sci U S A 86: 2612-2616

Kneussel M \& Wagner W (2013) Myosin motors at neuronal synapses: drivers of membrane transport and actin dynamics. Nat. Rev. Neurosci. 14: 233-247

Kofler K, Erdel M, Utermann G \& Baier G (2002) Molecular genetics and structural genomics of the human protein kinase C gene module. Genome Biol. 3: RESEARCH0014

Kohansal-Nodehi M, Chua JJ, Urlaub H, Jahn R \& Czernik D (2016) Analysis of protein phosphorylation in nerve terminal reveals extensive changes in active zone proteins upon exocytosis. eLife 5: e14530

Kononenko NL, Puchkov D, Classen GA, Walter AM, Pechstein A, Sawade L, Kaempf N, Trimbuch T, Lorenz D, Rosenmund C, Maritzen T \& Haucke V (2014) Clathrin/AP-2 mediate synaptic vesicle reformation from endosome-like vacuoles but are not essential for membrane retrieval at central synapses. Neuron 82: 981-988

Koos B, Andersson L, Clausson C-M, Grannas K, Klaesson A, Cane G \& Söderberg O (2014) Analysis of protein interactions in situ by proximity ligation assays. Curr. Top. Microbiol. Immunol. 377: 111126

Korogod N, Lou X \& Schneggenburger R (2007) Posttetanic potentiation critically depends on an enhanced $\mathrm{Ca} 2+$ sensitivity of vesicle fusion mediated by presynaptic PKC. Proceedings of the National Academy of Sciences 104: 15923-15928

Kraft AS, Anderson WB, Cooper HL \& Sando JJ (1982) Decrease in cytosolic calcium/phospholipiddependent protein kinase activity following phorbol ester treatment of EL4 thymoma cells. J. Biol. Chem. 257: 13193-13196

Krinner S, Butola T, Jung S, Wichmann C \& Moser T (2017) RIM-Binding Protein 2 Promotes a Large Number of CaV1.3 Ca2+-Channels and Contributes to Fast Synaptic Vesicle Replenishment at Hair Cell Active Zones. Front Cell Neurosci 11: 334

Kroll J, Jaime Tobón LM, Vogl C, Neef J, Kondratiuk I, König M, Strenzke N, Wichmann C, Milosevic I \& Moser T (2019) Endophilin-A regulates presynaptic Ca2+ influx and synaptic vesicle recycling in auditory hair cells. $E M B O J .38$ :

Le TL, Joseph SR, Yap AS \& Stow JL (2002) Protein kinase C regulates endocytosis and recycling of Ecadherin. Am. J. Physiol., Cell Physiol. 283: C489-499

Lee A, Westenbroek RE, Haeseleer F, Palczewski K, Scheuer T \& Catterall WA (2002) Differential modulation of $\mathrm{Ca}(\mathrm{v}) 2.1$ channels by calmodulin and $\mathrm{Ca} 2+-$ binding protein 1 . Nat. Neurosci 5: 210-217

Leenders AGM \& Sheng Z-H (2005) Modulation of neurotransmitter release by the second messengeractivated protein kinases: implications for presynaptic plasticity. Pharmacology \& therapeutics 105: 69-84

Lek A, Evesson FJ, Sutton RB, North KN \& Cooper ST (2012) Ferlins: Regulators of Vesicle Fusion for Auditory Neurotransmission, Receptor Trafficking and Membrane Repair. Traffic 13: 185-194 
Lek A, Lek M, North KN \& Cooper ST (2010) Phylogenetic analysis of ferlin genes reveals ancient eukaryotic origins. BMC Evol. Biol. 10: 231

Lenzi D \& von Gersdorff H (2001) Structure suggests function: the case for synaptic ribbons as exocytotic nanomachines. Bioessays 23: 831-840

Levic S, Bouleau Y \& Dulon D (2011) Developmental Acquisition of a Rapid Calcium-Regulated Vesicle Supply Allows Sustained High Rates of Exocytosis in Auditory Hair Cells. PLoS ONE 6: e25714

Li C, Ullrich B, Zhang JZ, Anderson RG, Brose N \& Südhof TC (1995) Ca(2+)-dependent and independent activities of neural and non-neural synaptotagmins. Nature 375: 594-599

Liu JP, Sim AT \& Robinson PJ (1994) Calcineurin inhibition of dynamin I GTPase activity coupled to nerve terminal depolarization. Science 265: 970-973

Llinás R, Gruner JA, Sugimori M, McGuinness TL \& Greengard P (1991) Regulation by synapsin I and $\mathrm{Ca}(2+)$-calmodulin-dependent protein kinase II of the transmitter release in squid giant synapse. $J$. Physiol. (Lond.) 436: 257-282

Llinás R, McGuinness TL, Leonard CS, Sugimori M \& Greengard P (1985) Intraterminal injection of synapsin I or calcium/calmodulin-dependent protein kinase II alters neurotransmitter release at the squid giant synapse. Proc. Natl. Acad. Sci. U.S.A. 82: 3035-3039

Loder MK \& Melikian HE (2003) The dopamine transporter constitutively internalizes and recycles in a protein kinase C-regulated manner in stably transfected PC12 cell lines. J. Biol. Chem. 278: 2216822174

Longo-Guess C, Gagnon LH, Bergstrom DE \& Johnson KR (2007) A missense mutation in the conserved C2B domain of otoferlin causes deafness in a new mouse model of DFNB9. Hear Res 234: 21-28

Lou X, Scheuss V \& Schneggenburger R (2005) Allosteric modulation of the presynaptic Ca2+ sensor for vesicle fusion. Nature 435: 497-501

Magupalli VG, Schwarz K, Alpadi K, Natarajan S, Seigel GM \& Schmitz F (2008) Multiple RIBEYERIBEYE interactions create a dynamic scaffold for the formation of synaptic ribbons. $J$ Neurosci 28: 7954-7967

Malenka RC (2003) The long-term potential of LTP. Nat. Rev. Neurosci. 4: 923-926

Malenka RC, Madison DV \& Nicoll RA (1986) Potentiation of synaptic transmission in the hippocampus by phorbol esters. Nature 321: 175-177

Mann ZF \& Kelley MW (2011) Development of tonotopy in the auditory periphery. Hearing Research 276: $2-15$

Marlin S, Feldmann D, Nguyen Y, Rouillon I, Loundon N, Jonard L, Bonnet C, Couderc R, Garabedian EN, Petit C \& Denoyelle F (2010) Temperature-sensitive auditory neuropathy associated with an otoferlin mutation: Deafening fever! Biochem. Biophys. Res. Commun 394: 737-742

Masuda M, Takeda S, Sone M, Ohki T, Mori H, Kamioka Y \& Mochizuki N (2006) Endophilin BAR domain drives membrane curvature by two newly identified structure-based mechanisms. $E M B O$ J. 25: 2889-2897 
Matsunaga T, Mutai H, Kunishima S, Namba K, Morimoto N, Shinjo Y, Arimoto Y, Kataoka Y, Shintani T, Morita N, Sugiuchi T, Masuda S, Nakano A, Taiji H \& Kaga K (2012) A prevalent founder mutation and genotype-phenotype correlations of OTOF in Japanese patients with auditory neuropathy. Clin. Genet. 82: 425-432

Matthew WD, Tsavaler L \& Reichardt LF (1981) Identification of a synaptic vesicle-specific membrane protein with a wide distribution in neuronal and neurosecretory tissue. J. Cell Biol. 91: 257-269

Matthews G \& Fuchs P (2010) The diverse roles of ribbon synapses in sensory neurotransmission. Nat Rev Neurosci 11: 812-822

Matthews G \& Sterling P (2008) Evidence that vesicles undergo compound fusion on the synaptic ribbon. The Journal of Neuroscience 28: 5403

Maxeiner S, Luo F, Tan A, Schmitz F \& Südhof TC (2016) How to make a synaptic ribbon: RIBEYE deletion abolishes ribbons in retinal synapses and disrupts neurotransmitter release. The EMBO Journal 35: 1098-1114

Maximov A \& Südhof TC (2005) Autonomous Function of Synaptotagmin 1 in Triggering Synchronous Release Independent of Asynchronous Release. Neuron 48: 547-554

McMahon HT, Missler M, Li C \& Südhof TC (1995) Complexins: cytosolic proteins that regulate SNAP receptor function. Cell 83: 111-119

Meese S (2015) Biochemical studies of the synaptic protein otoferlin. Available at: https://ediss.unigoettingen.de/handle/11858/00-1735-0000-0022-6089-2 [Accessed July 18, 2017]

Meese S, Cepeda AP, Gahlen F, Adams CM, Ficner R, Ricci AJ, Heller S, Reisinger E \& Herget M (2017) Activity-Dependent Phosphorylation by CaMKII $\delta$ Alters the Ca2+Affinity of the Multi-C2Domain Protein Otoferlin. Front Synaptic Neurosci 9: 13

Meyer AC, Frank T, Khimich D, Hoch G, Riedel D, Chapochnikov NM, Yarin YM, Harke B, Hell SW, Egner A \& Moser T (2009) Tuning of synapse number, structure and function in the cochlea. Nat Neurosci 12: 444-453

Michalski N, Goutman JD, Auclair SM, Monvel JB de, Tertrais M, Emptoz A, Parrin A, Nouaille S, Guillon M, Sachse M, Ciric D, Bahloul A, Hardelin J-P, Sutton RB, Avan P, Krishnakumar SS, Rothman JE, Dulon D, Safieddine S \& Petit C (2017) Otoferlin acts as a Ca2+ sensor for vesicle fusion and vesicle pool replenishment at auditory hair cell ribbon synapses. eLife Sciences 6: e31013

Michanski S, Smaluch K, Steyer AM, Chakrabarti R, Setz C, Oestreicher D, Fischer C, Möbius W, Moser T, Vogl C \& Wichmann C (2019) Mapping developmental maturation of inner hair cell ribbon synapses in the apical mouse cochlea. PNAS 116: 6415-6424

Milosevic I, Giovedi S, Lou X, Raimondi A, Collesi C, Shen H, Paradise S, O’Toole E, Ferguson S, Cremona O \& De Camilli P (2011) Recruitment of Endophilin to Clathrin-Coated Pit Necks Is Required for Efficient Vesicle Uncoating after Fission. Neuron 72: 587-601

Mirghomizadeh F, Pfister M, Apaydin F, Petit C, Kupka S, Pusch CM, Zenner HP \& Blin N (2002) Substitutions in the conserved C2C domain of otoferlin cause DFNB9, a form of nonsyndromic autosomal recessive deafness. Neurobiol. Dis 10: 157-164 
Moser T \& Beutner D (2000) Kinetics of exocytosis and endocytosis at the cochlear inner hair cell afferent synapse of the mouse. Proc Natl Acad Sci U S A 97: 883-888

Moser T, Predoehl F \& Starr A (2013) Review of hair cell synapse defects in sensorineural hearing impairment. Otol. Neurotol. 34: 995-1004

Moser T \& Starr A (2016) Auditory neuropathy — neural and synaptic mechanisms. Nat Rev Neurol 12: $135-149$

Mukherjee K, Yang X, Gerber SH, Kwon H-B, Ho A, Castillo PE, Liu X \& Südhof TC (2010) Piccolo and bassoon maintain synaptic vesicle clustering without directly participating in vesicle exocytosis. Proc. Natl. Acad. Sci. U.S.A. 107: 6504-6509

Müller A (2017) Molecular studies of the synaptic protein otoferlin. Available at: https://ediss.unigoettingen.de/handle/11858/00-1735-0000-0023-3DB5-0 [Accessed August 14, 2019]

Müller TM, Gierke K, Joachimsthaler A, Sticht H, Izsvák Z, Hamra FK, Fejtová A, Ackermann F, Garner CC, Kremers J, Brandstätter JH \& Regus-Leidig H (2019) A multiple Piccolino-RIBEYE interaction supports plate-shaped synaptic ribbons in retinal neurons. J. Neurosci.: 2038-18

Müller U \& Barr-Gillespie PG (2015) New treatment options for hearing loss. Nature Reviews Drug Discovery 14: 346-365

Muresan V, Lyass A \& Schnapp BJ (1999) The Kinesin Motor KIF3A Is a Component of the Presynaptic Ribbon in Vertebrate Photoreceptors. J. Neurosci. 19: 1027-1037

Nagy G, Matti U, Nehring RB, Binz T, Rettig J, Neher E \& Sørensen JB (2002) Protein kinase Cdependent phosphorylation of synaptosome-associated protein of $25 \mathrm{kDa}$ at Ser 187 potentiates vesicle recruitment. The Journal of neuroscience : the official journal of the Society for Neuroscience 22: 9278-86

Nalefski EA \& Falke JJ (1996) The C2 domain calcium-binding motif: structural and functional diversity. Protein Sci. 5: 2375-2390

Neef A, Heinemann C \& Moser T (2007a) Measurements of membrane patch capacitance using a softwarebased lock-in system. Pflugers Arch. 454: 335-344

Neef A, Khimich D, Pirih P, Riedel D, Wolf F \& Moser T (2007b) Probing the Mechanism of Exocytosis at the Hair Cell Ribbon Synapse. J. Neurosci. 27: 12933-12944

Neef J, Jung S, Wong AB, Reuter K, Pangrsic T, Chakrabarti R, Kugler S, Lenz C, Nouvian R, Boumil RM, Frankel WN, Wichmann C \& Moser T (2014) Modes and Regulation of Endocytic Membrane Retrieval in Mouse Auditory Hair Cells. J. Neurosci. 34: 705-716

Neher E \& Sakaba T (2008) Multiple roles of calcium ions in the regulation of neurotransmitter release. Neuron 59: 861-872

Newton AC (2010) Protein kinase C: poised to signal. Am. J. Physiol. Endocrinol. Metab. 298: E395-402

Nielander HB, Onofri F, Valtorta F, Schiavo G, Montecucco C, Greengard P \& Benfenati F (1995) Phosphorylation of VAMP/Synaptobrevin in Synaptic Vesicles by Endogenous Protein Kinases. Journal of Neurochemistry 65: 1712-1720 
Nishikawa K, Toker A, Johannes F-J, Songyang Z \& Cantley LC (1997) Determination of the Specific Substrate Sequence Motifs of Protein Kinase C Isozymes. J. Biol. Chem. 272: 952-960

Nishio S-Y \& Usami S-I (2017) Outcomes of cochlear implantation for the patients with specific genetic etiologies: a systematic literature review. Acta Otolaryngol. 137: 730-742

Nishizuka Y (1984) The role of protein kinase $C$ in cell surface signal transduction and tumour promotion. Nature 308: 693-698

Nouvian R, Beutner D, Parsons TD \& Moser T (2006) Structure and function of the hair cell ribbon synapse. The Journal of membrane biology 209: 153-165

Nouvian R, Neef J, Bulankina AV, Reisinger E, Pangršič T, Frank T, Sikorra S, Brose N, Binz T \& Moser $\mathrm{T}$ (2011) Exocytosis at the hair cell ribbon synapse apparently operates without neuronal SNARE proteins. Nat Neurosci 14: 411-413

Ohara-Imaizumi M, Ohtsuka T, Matsushima S, Akimoto Y, Nishiwaki C, Nakamichi Y, Kikuta T, Nagai S, Kawakami H, Watanabe T \& Nagamatsu S (2005) ELKS, a Protein Structurally Related to the Active Zone-associated Protein CAST, Is Expressed in Pancreatic $\beta$ Cells and Functions in Insulin Exocytosis: Interaction of ELKS with Exocytotic Machinery Analyzed by Total Internal Reflection Fluorescence Microscopy. Molecular Biology of the Cell 16: 3289-3300

Ohlemiller KK, Jones SM \& Johnson KR (2016) Application of Mouse Models to Research in Hearing and Balance. Journal of the Association for Research in Otolaryngology 17: 493-523

Ohtsuka T, Takao-Rikitsu E, Inoue E, Inoue M, Takeuchi M, Matsubara K, Deguchi-Tawarada M, Satoh K, Morimoto K, Nakanishi H \& Takai Y (2002) Cast: a novel protein of the cytomatrix at the active zone of synapses that forms a ternary complex with RIM1 and munc13-1. J Cell Biol 158: $577-590$

Ohyama A, Hosaka K, Komiya Y, Akagawa K, Yamauchi E, Taniguchi H, Sasagawa N, Kumakura K, Mochida S, Yamauchi T \& Igarashi M (2002) Regulation of exocytosis through Ca2+/ATPdependent binding of autophosphorylated $\mathrm{Ca} 2+/$ calmodulin-activated protein kinase II to syntaxin 1A. J. Neurosci. 22: 3342-3351

Olofsson B, Chardin P, Touchot N, Zahraoui A \& Tavitian A (1988) Expression of the ras-related ralA, rho12 and rab genes in adult mouse tissues. Oncogene 3: 231-234

Padmanarayana M, Hams N, Speight LC, Petersson EJ, Mehl RA \& Johnson CP (2014) Characterization of the lipid binding properties of Otoferlin reveals specific interactions between PI(4,5)P2 and the C2C and C2F domains. Biochemistry 53: 5023-5033

Pang ZP, Cao P, Xu W \& Südhof TC (2010) Calmodulin controls synaptic strength via presynaptic activation of calmodulin kinase II. J. Neurosci. 30: 4132-4142

Pangršič T, Gabrielaitis M, Michanski S, Schwaller B, Wolf F, Strenzke N \& Moser T (2015) EF-hand protein $\mathrm{Ca} 2+$ buffers regulate $\mathrm{Ca} 2+$ influx and exocytosis in sensory hair cells. PNAS 112: E1028E1037

Pangršič T, Lasarow L, Reuter K, Takago H, Schwander M, Riedel D, Frank T, Tarantino LM, Bailey JS, Strenzke N, Brose N, Müller U, Reisinger E \& Moser T (2010) Hearing requires otoferlin- 
dependent efficient replenishment of synaptic vesicles in hair cells. Nature Neuroscience 13: 869876

Pangrsic T, Lasarow L, Reuter K, Takago H, Schwander M, Riedel D, Frank T, Tarantino LM, Bailey JS, Strenzke N, Brose N, Müller U, Reisinger E \& Moser T (2010) Hearing requires otoferlindependent efficient replenishment of synaptic vesicles in hair cells. Nat. Neurosci. 13: 869-876

Pangršič T, Reisinger E \& Moser T (2012) Otoferlin: a multi-C2 domain protein essential for hearing. Trends Neurosci. 35: 671-680

Pangrsic T \& Vogl C (2018) Balancing presynaptic release and endocytic membrane retrieval at hair cell ribbon synapses. FEBS Letters 0 : Available at: https://febs.onlinelibrary.wiley.com/doi/abs/10.1002/1873-3468.13258 [Accessed November 5, 2018]

Parfitt KD \& Madison DV (1993) Phorbol esters enhance synaptic transmission by a presynaptic, calciumdependent mechanism in rat hippocampus. J. Physiol. (Lond.) 471: 245-268

Parker PJ \& Murray-Rust J (2004) PKC at a glance. J. Cell. Sci. 117: 131-132

Parsons TD \& Sterling P (2003) Synaptic ribbon. Conveyor belt or safety belt? Neuron 37: 379-382

Peng Z, Grimberg E \& Sagi-Eisenberg R (2002) Suppression of Synaptotagmin II restrains phorbolesterinduced downregulation of protein kinase Calpha by diverting the kinase from a degradative pathway to the recycling endocytic compartment. J. Cell. Sci. 115: 3083-3092

Pepio AM \& Sossin WS (2001) Membrane translocation of novel protein kinase Cs is regulated by phosphorylation of the C2 domain. J. Biol. Chem. 276: 3846-3855

Perez-Riverol Y, Csordas A, Bai J, Bernal-Llinares M, Hewapathirana S, Kundu DJ, Inuganti A, Griss J, Mayer G, Eisenacher M, Pérez E, Uszkoreit J, Pfeuffer J, Sachsenberg T, Yilmaz S, Tiwary S, Cox J, Audain E, Walzer M, Jarnuczak AF, et al (2019) The PRIDE database and related tools and resources in 2019: improving support for quantification data. Nucleic Acids Res. 47: D442-D450

Perin MS, Fried VA, Mignery GA, Jahn R \& S|[uuml]|dhof TC (1990) Phospholipid binding by a synaptic vesicle protein homologous to the regulatory region of protein kinase C. Nature 345: 260-263

Picher MM, Gehrt A, Meese S, Ivanovic A, Predoehl F, Jung S, Schrauwen I, Dragonetti AG, Colombo R, Camp GV, Strenzke N \& Moser T (2017a) Ca2+-binding protein 2 inhibits Ca2+-channel inactivation in mouse inner hair cells. PNAS 114: E1717-E1726

Picher MM, Oprişoreanu A-M, Jung S, Michel K, Schoch S \& Moser T (2017b) Rab Interacting Molecules 2 and 3 Directly Interact with the Pore-Forming CaV1.3 Ca2+ Channel Subunit and Promote Its Membrane Expression. Front Cell Neurosci 11: Available at: http://www.ncbi.nlm.nih.gov/pmc/articles/PMC5462952/ [Accessed July 3, 2017]

Platzer J, Engel J, Schrott-Fischer A, Stephan K, Bova S, Chen H, Zheng H \& Striessnig J (2000) Congenital deafness and sinoatrial node dysfunction in mice lacking class D L-type Ca2+ channels. Cell 102: 89-97 
Popoli M (1993) Synaptotagmin is endogenously phosphorylated by Ca2+/calmodulin protein kinase II in synaptic vesicles. FEBS Letters 317: 85-88

Qu L, Akbergenova Y, Hu Y \& Schikorski T (2009) Synapse-to-synapse variation in mean synaptic vesicle size and its relationship with synaptic morphology and function. J. Comp. Neurol. 514: 343-352

Radhakrishnan A, Stein A, Jahn R \& Fasshauer D (2009) The Ca2+ affinity of synaptotagmin 1 is markedly increased by a specific interaction of its C2B domain with phosphatidylinositol 4,5-bisphosphate. J. Biol. Chem. 284: 25749-25760

Ramakrishnan NA, Drescher MJ \& Drescher DG (2009) Direct interaction of otoferlin with syntaxin 1A, SNAP-25, and the L-type voltage-gated calcium channel Cav1.3. J. Biol. Chem 284: 1364-1372

Ramakrishnan NA, Drescher MJ, Morley BJ, Kelley PM \& Drescher DG (2014) Calcium Regulates Molecular Interactions of Otoferlin with SNARE Proteins Required for Hair Cell Exocytosis. Journal of Biological Chemistry Available at: http://www.jbc.org/cgi/doi/10.1074/jbc.M113.480533 [Accessed February 3, 2014]

Redpath GMI, Sophocleous RA, Turnbull L, Whitchurch CB \& Cooper ST (2015) Ferlins show tissuespecific expression and segregate as plasma membrane/late endosomal or trans-Golgi/recycling ferlins. Traffic: $\mathrm{n} / \mathrm{a}-\mathrm{n} / \mathrm{a}$

Regus-Leidig H, Ott C, Löhner M, Atorf J, Fuchs M, Sedmak T, Kremers J, Fejtová A, Gundelfinger ED \& Brandstätter JH (2013) Identification and Immunocytochemical Characterization of Piccolino, a Novel Piccolo Splice Variant Selectively Expressed at Sensory Ribbon Synapses of the Eye and Ear. PLoS ONE 8: e70373

Reim K, Mansour M, Varoqueaux F, McMahon HT, Südhof TC, Brose N \& Rosenmund C (2001) Complexins regulate a late step in Ca2+-dependent neurotransmitter release. Cell 104: 71-81

Reisinger E, Bresee C, Neef J, Nair R, Reuter K, Bulankina A, Nouvian R, Koch M, Bückers J, Kastrup L, Roux I, Petit C, Hell SW, Brose N, Rhee J-S, Kügler S, Brigande JV \& Moser T (2011) Probing the functional equivalence of otoferlin and synaptotagmin 1 in exocytosis. J. Neurosci. 31: 48864895

Revelo NH, Kamin D, Truckenbrodt S, Wong AB, Reuter-Jessen K, Reisinger E, Moser T \& Rizzoli SO (2014) A new probe for super-resolution imaging of membranes elucidates trafficking pathways. $J$. Cell Biol. 205: 591-606

Rhee J-S, Betz A, Pyott S, Reim K, Varoqueaux F, Augustin I, Hesse D, Südhof TC, Takahashi M, Rosenmund C \& Brose N (2002) [beta] Phorbol Ester- and Diacylglycerol-Induced Augmentation of Transmitter Release Is Mediated by Munc13s and Not by PKCs. Cell 108: 121-133

Rizo J \& Rosenmund C (2008) Synaptic vesicle fusion. Nat. Struct. Mol. Biol. 15: 665-674

Rizzoli SO \& Betz WJ (2005) Synaptic vesicle pools. Nat. Rev. Neurosci. 6: 57-69

Roberts WM (1994) Localization of calcium signals by a mobile calcium buffer in frog saccular hair cells. J. Neurosci 14: 3246-3262 
Roggero CM, Tomes CN, De Blas GA, Castillo J, Michaut MA, Fukuda M \& Mayorga LS (2005) Protein kinase C-mediated phosphorylation of the two polybasic regions of synaptotagmin VI regulates their function in acrosomal exocytosis. Dev. Biol. 285: 422-435

Rosenberg OS, Deindl S, Sung R-J, Nairn AC \& Kuriyan J (2005) Structure of the autoinhibited kinase domain of CaMKII and SAXS analysis of the holoenzyme. Cell 123: 849-860

Rosse C, Linch M, Kermorgant S, Cameron AJM, Boeckeler K \& Parker PJ (2010) PKC and the control of localized signal dynamics. Nat. Rev. Mol. Cell Biol. 11: 103-112

Roux I, Hosie S, Johnson SL, Bahloul A, Cayet N, Nouaille S, Kros CJ, Petit C \& Safieddine S (2009) Myosin VI is required for the proper maturation and function of inner hair cell ribbon synapses. Hum. Mol. Genet. 18: 4615-4628

Roux I, Safieddine S, Nouvian R, Grati M, Simmler M-C, Bahloul A, Perfettini I, Le Gall M, Rostaing P, Hamard G, Triller A, Avan P, Moser T \& Petit C (2006) Otoferlin, defective in a human deafness form, is essential for exocytosis at the auditory ribbon synapse. Cell 127: 277-289

Ruel J, Emery S, Nouvian R, Bersot T, Amilhon B, Van Rybroek JM, Rebillard G, Lenoir M, Eybalin M, Delprat B, Sivakumaran TA, Giros B, El Mestikawy S, Moser T, Smith RJH, Lesperance MM \& Puel J-L (2008) Impairment of SLC17A8 Encoding Vesicular Glutamate Transporter-3, VGLUT3, Underlies Nonsyndromic Deafness DFNA25 and Inner Hair Cell Dysfunction in Null Mice. Am J Hum Genet 83: 278-292

Rutherford MA \& Pangršič T (2012) Molecular anatomy and physiology of exocytosis in sensory hair cells. Cell calcium 52: 327-37

Ryan TA, Li L, Chin LS, Greengard P \& Smith SJ (1996) Synaptic vesicle recycling in synapsin I knockout mice. J. Cell Biol. 134: 1219-1227

Safieddine S, El-Amraoui A \& Petit C (2012) The auditory hair cell ribbon synapse: from assembly to function. Annu Rev Neurosci 35: 509-528

Safieddine S \& Wenthold RJ (1999) SNARE complex at the ribbon synapses of cochlear hair cells: analysis of synaptic vesicle- and synaptic membrane-associated proteins. European Journal of Neuroscience 11: $803-812$

Sakai N, Sasaki K, Ikegaki N, Shirai Y, Ono Y \& Saito N (1997) Direct visualization of the translocation of the gamma-subspecies of protein kinase $\mathrm{C}$ in living cells using fusion proteins with green fluorescent protein. J. Cell Biol. 139: 1465-1476

Sánchez-Bautista S, Marín-Vicente C, Gómez-Fernández JC \& Corbalán-García S (2006) The C2 domain of PKCalpha is a Ca2+-dependent PtdIns(4,5)P2 sensing domain: a new insight into an old pathway. J. Mol. Biol. 362: 901-914

Schechtman D, Craske ML, Kheifets V, Meyer T, Schechtman J \& Mochly-Rosen D (2004) A critical intramolecular interaction for protein kinase Cepsilon translocation. J. Biol. Chem. 279: 1583115840 
Schindelin J, Arganda-Carreras I, Frise E, Kaynig V, Longair M, Pietzsch T, Preibisch S, Rueden C, Saalfeld S, Schmid B, Tinevez J-Y, White DJ, Hartenstein V, Eliceiri K, Tomancak P \& Cardona A (2012) Fiji: an open-source platform for biological-image analysis. Nat. Methods 9: 676-682

Schmitz F (2009) The Making of Synaptic Ribbons: How They Are Built and What They Do. The Neuroscientist 15: 611-624

Schmitz F, Königstorfer A \& Südhof TC (2000) RIBEYE, a component of synaptic ribbons: a protein's journey through evolution provides insight into synaptic ribbon function. Neuron 28: 857-872

Schnee ME, Santos-Sacchi J, Castellano-Muñoz M, Kong J-H \& Ricci AJ (2011) Calcium-Dependent Synaptic Vesicle Trafficking Underlies Indefatigable Release at the Hair Cell Afferent Fiber Synapse. Neuron 70: 326-338

Schoch S, Castillo PE, Jo T, Mukherjee K, Geppert M, Wang Y, Schmitz F, Malenka RC \& Südhof TC (2002) RIM1alpha forms a protein scaffold for regulating neurotransmitter release at the active zone. Nature 415: 321-326

Schug N, Braig C, Zimmermann U, Engel J, Winter H, Ruth P, Blin N, Pfister M, Kalbacher H \& Knipper M (2006) Differential expression of otoferlin in brain, vestibular system, immature and mature cochlea of the rat. European Journal of Neuroscience 24: 3372-3380

Schwander M, Sczaniecka A, Grillet N, Bailey JS, Avenarius M, Najmabadi H, Steffy BM, Federe GC, Lagler EA, Banan R, Hice R, Grabowski-Boase L, Keithley EM, Ryan AF, Housley GD, Wiltshire T, Smith RJH, Tarantino LM \& Müller U (2007) A Forward Genetics Screen in Mice Identifies Recessive Deafness Traits and Reveals That Pejvakin Is Essential for Outer Hair Cell Function. J. Neurosci. 27: 2163-2175

Seal RP, Akil O, Yi E, Weber CM, Grant L, Yoo J, Clause A, Kandler K, Noebels JL, Glowatzki E, Lustig LR \& Edwards RH (2008) Sensorineural Deafness and Seizures in Mice Lacking Vesicular Glutamate Transporter 3. Neuron 57: 263-275

Shapira R, Silberberg SD, Ginsburg S \& Rahamimoff R (1987) Activation of protein kinase C augments evoked transmitter release. Nature 325: 58-60

Shearer AE \& Smith RJH (2015) Massively Parallel Sequencing for Genetic Diagnosis of Hearing Loss: The New Standard of Care. Otolaryngol Head Neck Surg 153: 175-182

Sheets L, Trapani JG, Mo W, Obholzer N \& Nicolson T (2011) Ribeye is required for presynaptic CaV1.3a channel localization and afferent innervation of sensory hair cells. Development 138: 1309-1319

Shimazaki Y, Nishiki T, Omori A, Sekiguchi M, Kamata Y, Kozaki S \& Takahashi M (1996) Phosphorylation of $25-\mathrm{kDa}$ synaptosome-associated protein. Possible involvement in protein kinase C-mediated regulation of neurotransmitter release. The Journal of biological chemistry 271: $14548-53$

Shupliakov O, Haucke V \& Pechstein A (2011) How synapsin I may cluster synaptic vesicles. Seminars in Cell \& Developmental Biology 22: 393-399 
Söllner T, Bennett MK, Whiteheart SW, Scheller RH \& Rothman JE (1993) A protein assemblydisassembly pathway in vitro that may correspond to sequential steps of synaptic vesicle docking, activation, and fusion. Cell 75: 409-418

Sørensen JB (2009) Conflicting Views on the Membrane Fusion Machinery and the Fusion Pore. Annual Review of Cell and Developmental Biology 25: 513-537

Sørensen JB, Fernández-Chacón R, Südhof TC \& Neher E (2003) Examining synaptotagmin 1 function in dense core vesicle exocytosis under direct control of Ca2+. J. Gen. Physiol. 122: 265-276

Starr A, Picton TW, Sininger Y, Hood LJ \& Berlin CI (1996) Auditory neuropathy. Brain 119: 741-754

Sterling P \& Matthews G (2005) Structure and function of ribbon synapses. Trends in Neurosciences 28: $20-29$

Stevens CF \& Sullivan JM (1998) Regulation of the readily releasable vesicle pool by protein kinase C. Neuron 21: 885-893

Strenzke N, Chakrabarti R, Al-Moyed H, Müller A, Hoch G, Pangrsic T, Yamanbaeva G, Lenz C, Pan KT, Auge E, Geiss-Friedlander R, Urlaub H, Brose N, Wichmann C \& Reisinger E (2016) Hair cell synaptic dysfunction, auditory fatigue and thermal sensitivity in otoferlin Ile515Thr mutants. EMBO J. 35: e201694564

Strenzke N, Chanda S, Kopp-Scheinpflug C, Khimich D, Reim K, Bulankina AV, Neef A, Wolf F, Brose N, Xu-Friedman MA \& Moser T (2009) Complexin-I Is Required for High-Fidelity Transmission at the Endbulb of Held Auditory Synapse. J. Neurosci. 29: 7991-8004

Südhof TC (1995) The synaptic vesicle cycle: a cascade of protein-protein interactions. Nature 375: 645653

Südhof TC (2013) Neurotransmitter release: the last millisecond in the life of a synaptic vesicle. Neuron 80: 675-690

Südhof TC (2014) The molecular machinery of neurotransmitter release (Nobel lecture). Angew. Chem. Int. Ed. Engl. 53: 12696-12717

Sun P, Enslen H, Myung PS \& Maurer RA (1994) Differential activation of CREB by Ca2+/calmodulindependent protein kinases type II and type IV involves phosphorylation of a site that negatively regulates activity. Genes Dev. 8: 2527-2539

Sutton RB, Davletov BA, Berghuis AM, Südhof TC \& Sprang SR (1995) Structure of the first C2 domain of synaptotagmin I: a novel Ca2+/phospholipid-binding fold. Cell 80: 929-938

Takahashi S, Yamamoto H, Matsuda Z, Ogawa M, Yagyu K, Taniguchi T, Miyata T, Kaba H, Higuchi T, Okutani F \& Fujimoto S (1995) Identification of two highly homologous presynaptic proteins distinctly localized at the dendritic and somatic synapses. FEBS Letters 368: 455-460

Takamori S, Rhee JS, Rosenmund C \& Jahn R (2001) Identification of differentiation-associated brainspecific phosphate transporter as a second vesicular glutamate transporter (VGLUT2). J. Neurosci. 21: RC182 
Takekoshi S, Kambayashi Y, Nagata H, Takagi T, Yamamoto Y \& Watanabe K (1995) Activation of Protein Kinase $\mathrm{C}$ by Oxidized Diacylglycerols. Biochemical and Biophysical Research Communications 217: 654-660

Tardif M, Savard M, Flamand L \& Gosselin J (2002) Impaired protein kinase C activation/translocation in Epstein-Barr virus-infected monocytes. J. Biol. Chem. 277: 24148-24154

Tertrais M, Bouleau Y, Emptoz A, Belleudy S, Sutton RB, Petit C, Safieddine S \& Dulon D (2019) Viral Transfer of Mini-Otoferlins Partially Restores the Fast Component of Exocytosis and Uncovers Ultrafast Endocytosis in Auditory Hair Cells of Otoferlin Knock-Out Mice. J. Neurosci. 39: 33943411

Tobimatsu T \& Fujisawa H (1989) Tissue-specific expression of four types of rat calmodulin-dependent protein kinase II mRNAs. J. Biol. Chem. 264: 17907-17912

Tombes RM, Faison MO \& Turbeville JM (2003) Organization and evolution of multifunctional $\mathrm{Ca}(2+) / \mathrm{CaM}-$ dependent protein kinase genes. Gene 322: 17-31

Truckenbrodt S, Viplav A, Jähne S, Vogts A, Denker A, Wildhagen H, Fornasiero EF \& Rizzoli SO (2018) Newly produced synaptic vesicle proteins are preferentially used in synaptic transmission. EMBO J. 37:

Tumbarello DA, Kendrick-Jones J \& Buss F (2013) Myosin VI and its cargo adaptors - linking endocytosis and autophagy. J. Cell. Sci. 126: 2561-2570

Turner KM, Burgoyne RD \& Morgan A (1999) Protein phosphorylation and the regulation of synaptic membrane traffic. Trends Neurosci. 22: 459-464

Uthaiah RC \& Hudspeth AJ (2010) Molecular Anatomy of the Hair Cell's Ribbon Synapse. J. Neurosci. 30: $12387-12399$

Varga R, Avenarius MR, Kelley PM, Keats BJ, Berlin CI, Hood LJ, Morlet TG, Brashears SM, Starr A, Cohn ES, Smith RJH \& Kimberling WJ (2006) OTOF mutations revealed by genetic analysis of hearing loss families including a potential temperature sensitive auditory neuropathy allele. $J$ Med Genet 43: 576-581

Varga R, Kelley PM, Keats BJ, Starr A, Leal SM, Cohn E \& Kimberling WJ (2003) Non-syndromic recessive auditory neuropathy is the result of mutations in the otoferlin (OTOF) gene. J. Med. Genet. 40: 45-50

Verdaguer N, Corbalan-Garcia S, Ochoa WF, Fita I \& Gómez-Fernández JC (1999) Ca(2+) bridges the $\mathrm{C} 2$ membrane-binding domain of protein kinase Calpha directly to phosphatidylserine. $E M B O J$. 18: 6329-6338

Verhage M, Maia AS, Plomp JJ, Brussaard AB, Heeroma JH, Vermeer H, Toonen RF, Hammer RE, Den TK van, Berg, Missler M, Geuze HJ \& Südhof TC (2000) Synaptic Assembly of the Brain in the Absence of Neurotransmitter Secretion. Science 287: 864-869

Vilardi F, Lorenz H \& Dobberstein B (2011) WRB is the receptor for TRC40/Asna1-mediated insertion of tail-anchored proteins into the ER membrane. J. Cell. Sci. 124: 1301-1307 
Vincent PF, Bouleau Y, Petit C \& Dulon D (2015) A synaptic F-actin network controls otoferlindependent exocytosis in auditory inner hair cells. eLife 4: Available at: http://www.ncbi.nlm.nih.gov/pmc/articles/PMC4714970/ [Accessed April 29, 2016]

Vincent PFY, Bouleau Y, Charpentier G, Emptoz A, Safieddine S, Petit C \& Dulon D (2017) Different CaV1.3 Channel Isoforms Control Distinct Components of the Synaptic Vesicle Cycle in Auditory Inner Hair Cells. J. Neurosci. 37: 2960-2975

Vincent PFY, Bouleau Y, Safieddine S, Petit C \& Dulon D (2014) Exocytotic machineries of vestibular type I and cochlear ribbon synapses display similar intrinsic otoferlin-dependent $\mathrm{Ca} 2+$ sensitivity but a different coupling to Ca2+ channels. J. Neurosci. 34: 10853-10869

Voets T, Moser T, Lund PE, Chow RH, Geppert M, Südhof TC \& Neher E (2001) Intracellular calcium dependence of large dense-core vesicle exocytosis in the absence of synaptotagmin I. Proc. Natl. Acad. Sci. U.S.A 98: 11680-11685

Vogl C, Cooper BH, Neef J, Wojcik SM, Reim K, Reisinger E, Brose N, Rhee J-S, Moser T \& Wichmann C (2015) Unconventional molecular regulation of synaptic vesicle replenishment in cochlear inner hair cells. J. Cell. Sci. 128: 638-644

Vogl C, Panou I, Yamanbaeva G, Wichmann C, Mangosing SJ, Vilardi F, Indzhykulian AA, Pangršič T, Santarelli R, Rodriguez-Ballesteros M, Weber T, Jung S, Cardenas E, Wu X, Wojcik SM, Kwan KY, Castillo I del, Schwappach B, Strenzke N, Corey DP, et al (2016) Tryptophan-rich basic protein (WRB) mediates insertion of the tail-anchored protein otoferlin and is required for hair cell exocytosis and hearing. EMBO J:: e201593565

Walent JH, Porter BW \& Martin TF (1992) A novel $145 \mathrm{kd}$ brain cytosolic protein reconstitutes Ca(2+)regulated secretion in permeable neuroendocrine cells. Cell 70: 765-775

Wang J, Bello O, Auclair SM, Wang J, Coleman J, Pincet F, Krishnakumar SS, Sindelar CV \& Rothman JE (2014) Calcium sensitive ring-like oligomers formed by synaptotagmin. Proc. Natl. Acad. Sci. U.S.A. 111: 13966-13971

Wang Y, Okamoto M, Schmitz F, Hofmann K \& Südhof TC (1997) Rim is a putative Rab3 effector in regulating synaptic-vesicle fusion. Nature 388: 593-598

Watanabe S \& Boucrot E (2017) Fast and ultrafast endocytosis. Curr. Opin. Cell Biol. 47: 64-71

Watanabe S, Mamer LE, Raychaudhuri S, Luvsanjav D, Eisen J, Trimbuch T, Söhl-Kielczynski B, Fenske P, Milosevic I, Rosenmund C \& Jorgensen EM (2018) Synaptojanin and Endophilin Mediate Neck Formation during Ultrafast Endocytosis. Neuron 98: 1184-1197.e6

Watanabe S, Rost BR, Camacho-Pérez M, Davis MW, Söhl-Kielczynski B, Rosenmund C \& Jorgensen EM (2013) Ultrafast endocytosis at mouse hippocampal synapses. Nature 504: 242-247

Watanabe S, Trimbuch T, Camacho-Pérez M, Rost BR, Brokowski B, Söhl-Kielczynski B, Felies A, Davis MW, Rosenmund C \& Jorgensen EM (2014) Clathrin regenerates synaptic vesicles from endosomes. Nature 515: 228-233 
White RR, Kwon YG, Taing M, Lawrence DS \& Edelman AM (1998) Definition of optimal substrate recognition motifs of $\mathrm{Ca} 2+$-calmodulin-dependent protein kinases IV and II reveals shared and distinctive features. J. Biol. Chem. 273: 3166-3172

Wichmann C \& Moser T (2015) Relating structure and function of inner hair cell ribbon synapses. Cell Tissue Res.

Wierda KDB, Toonen RFG, de Wit H, Brussaard AB \& Verhage M (2007) Interdependence of PKCdependent and PKC-independent pathways for presynaptic plasticity. Neuron 54: 275-90

Wong AB, Rutherford MA, Gabrielaitis M, Pangršič T, Göttfert F, Frank T, Michanski S, Hell S, Wolf F, Wichmann C \& Moser T (2014) Developmental refinement of hair cell synapses tightens the coupling of Ca2+ influx to exocytosis. EMBO J 33: 247-264

Wu L-G, Ryan TA \& Lagnado L (2007) Modes of Vesicle Retrieval at Ribbon Synapses, Calyx-Type Synapses, and Small Central Synapses. Journal of Neuroscience 27: 11793-11802

Wu X, Hu S, Kang X \& Wang C (2019) Synaptotagmins: Beyond Presynaptic Neurotransmitter Release. Neuroscientist: 1073858419844497

Wu X, Zhang X, Li X, Cheng H, Kuai Y, Wang S \& Guo Y (2006) Translocation of classical PKC and cortical granule exocytosis of human oocyte in germinal vesicle and metaphase II stage. Acta Pharmacol. Sin. 27: 1353-1358

Xue Y, Li A, Wang L, Feng H \& Yao X (2006) PPSP: prediction of PK-specific phosphorylation site with Bayesian decision theory. BMC Bioinformatics 7: 163

Xue Y, Liu Z, Cao J, Ma Q, Gao X, Wang Q, Jin C, Zhou Y, Wen L \& Ren J (2011) GPS 2.1: enhanced prediction of kinase-specific phosphorylation sites with an algorithm of motif length selection. Protein Eng. Des. Sel. 24: 255-260

Yamamoto Y \& Sakisaka T (2012) Molecular Machinery for Insertion of Tail-Anchored Membrane Proteins into the Endoplasmic Reticulum Membrane in Mammalian Cells. Mol. Cell 48: 387-397

Yang PS, Alseikhan BA, Hiel H, Grant L, Mori MX, Yang W, Fuchs PA \& Yue DT (2006) Switching of $\mathrm{Ca} 2+-$ dependent inactivation of $\mathrm{Ca}(\mathrm{v}) 1.3$ channels by calcium binding proteins of auditory hair cells. J. Neurosci 26: 10677-10689

Yang T, Scholl ES, Pan N, Fritzsch B, Haeseleer F \& Lee A (2016) Expression and Localization of CaBP $\mathrm{Ca} 2+$ Binding Proteins in the Mouse Cochlea. PLoS One 11: Available at: http://www.ncbi.nlm.nih.gov/pmc/articles/PMC4725724/ [Accessed February 15, 2016]

Yasunaga S, Grati M, Chardenoux S, Smith TN, Friedman TB, Lalwani AK, Wilcox ER \& Petit C (2000) OTOF Encodes Multiple Long and Short Isoforms: Genetic Evidence That the Long Ones Underlie Recessive Deafness DFNB9. Am J Hum Genet 67: 591-600

Yasunaga S, Grati M, Cohen-Salmon M, El-Amraoui A, Mustapha M, Salem N, El-Zir E, Loiselet J \& Petit C (1999) A mutation in OTOF, encoding otoferlin, a FER-1-like protein, causes DFNB9, a nonsyndromic form of deafness. Nat Genet 21: 363-369 
144 | References

Yawo H (1999) Protein kinase C potentiates transmitter release from the chick ciliary presynaptic terminal by increasing the exocytotic fusion probability. J. Physiol. (Lond.) 515 ( Pt 1): 169-180

Yoshihara M \& Littleton JT (2002) Synaptotagmin I Functions as a Calcium Sensor to Synchronize Neurotransmitter Release. Neuron 36: 897-908

Zak M, Breß A, Brandt N, Franz C, Ruth P, Pfister M, Knipper M \& Blin N (2012) Ergic2, a brain specific interacting partner of otoferlin. Cell. Physiol. Biochem. 29: 941-948

Zhang B, Koh YH, Beckstead RB, Budnik V, Ganetzky B \& Bellen HJ (1998) Synaptic Vesicle Size and Number Are Regulated by a Clathrin Adaptor Protein Required for Endocytosis. Neuron 21: $1465-1475$

Zhang JZ, Davletov BA, Südhof TC \& Anderson RG (1994) Synaptotagmin I is a high affinity receptor for clathrin AP-2: implications for membrane recycling. Cell 78: 751-760

Zhao Y, Leal K, Abi-Farah C, Martin KC, Sossin WS \& Klein M (2006) Isoform specificity of PKC translocation in living Aplysia sensory neurons and a role for Ca2+-dependent PKC APL I in the induction of intermediate-term facilitation. J. Neurosci. 26: 8847-8856 


\section{Appendix}

Publication: Meese et al (2017)

The results presented in chapters 2.3.1 and 2.3.2 were published in Frontiers in Synaptic Neuroscience (C) as Meese et al, 2017:

Meese S, Cepeda AP, Gahlen F, Adams CM, Ficner R, Ricci AJ, Heller S, Reisinger E \& Herget M (2017) Activity-Dependent Phosphorylation by CaMKII $\delta$ Alters the $\mathrm{Ca}^{2+}$ Affinity of the Multi$\mathrm{C}_{2}$-Domain Protein Otoferlin. Front Synaptic Neurosci 9: 13.

See attached publication in the following pages. 
146 | Appendix

OPEN ACCESS

Edited by:

Martín Cammarota,

Federal University of Rio Grande do

Norte, Brazil

Reviewed by:

Ayse Dosemeci,

National Institutes of Health (N/H)

United States

Noelia Weisstaub,

Institute of Cognitive and Translational

Neuroscience (INCYT), Argentina

${ }^{*}$ Correspondence:

Meike Herge

meikeher@gmail.com

Ellen Reisinger

ellen.reisinger@meduni

goettingen.de

tPresent address Meike Herget,

Avails Medical, Inc., Menlo Park, CA

United States

Received: 21 July 2017 Accepted: 11 September 2017

Published: 04 October 2017

Citation:

Meese $S$, Cepeda AP, Gahlen F

Adams CM, Ficner $R$, Ricci AJ, Heller $S$, Reisinger $E$ and Herget $M$

(2017) Activity-Dependent

Phosphorylation by CaMKIIS Alters the $\mathrm{Ca}^{2+}$ Affinity of the

Multi-C2-Domain Protein Otoferlin.

Front. Synaptic Neurosci. 9:13

doi: 10.3389/fnsyn.2017.00013

\section{Activity-Dependent Phosphorylation by CaMKIII Alters the $\mathrm{Ca}^{2+}$ Affinity of the Multi- $\mathrm{C}_{2}$-Domain Protein Otoferlin}

\author{
Sandra Meese 1,2, Andreia P. Cepeda ${ }^{2,3}$, Felix Gahlen', Christopher M. Adams ${ }^{5}$, \\ Ralf Ficner ${ }^{1}$, Anthony J. Ricci ${ }^{6}$, Stefan Heller ${ }^{6}$, Ellen Reisinger ${ }^{3 *}$ and Meike Herget ${ }^{6+*}$ \\ ${ }^{1}$ Department of Molecular Structural Biology, Institute for Microbiology and Genetics, and Collaborative Research Center \\ 889, University of Göttingen, Göttingen, Germany, ${ }^{2}$ Göttingen Graduate School for Neurosciences, Biophysics, and \\ Molecular Biosciences, Göttingen, Germany, ${ }^{3}$ Molecular Biology of Cochlear Neurotransmission Group, Department of \\ Otorhinolaryngology, University Medical Center Göttingen, and Collaborative Research Center 889, University of Göttingen, \\ Göttingen, Germany, ${ }^{4}$ Department of Otorhinolaryngology, Head and Neck Surgery, Ruhr-University Bochum, Bochum. \\ Germany, ${ }^{5}$ Stanford University Mass Spectrometry, Stanford University, Stanford, CA, United States, ${ }^{6}$ Depariment of \\ Otolaryngology, Head and Neck Surgery, Stanford School of Medicine, Stanford, CA, United States
}

Otoferlin is essential for fast $\mathrm{Ca}^{2+}$-triggered transmitter release from auditory inner hair cells $(\mathrm{IHCS})$, playing key roles in synaptic vesicle release, replenishment and retrieval. Dysfunction of otoferlin results in profound prelingual deafness. Despite its crucial role in cochlear synaptic processes, mechanisms regulating otoferlin activity have not been studied to date. Here, we identified $\mathrm{Ca}^{2+} /$ calmodulin-dependent serine/threonine kinase II delta (CaMKIII) as an otoferlin binding partner by pull-downs from chicken utricles and reassured interaction by a co-immunoprecipitation with heterologously expressed proteins in HEK cells. We confirmed the expression of CaMKIII in rodent IHCs by immunohistochemistry and real-time PCR. A proximity ligation assay indicates close proximity of the two proteins in rat $\mathrm{HCS}$, suggesting that otoferlin and CaMKIll $\delta$ also interact in mammalian IHCs. In vitro phosphorylation of otoferlin by CaMKIII revealed ten phosphorylation sites, five of which are located within $\mathrm{C}_{2}$-domains. Exchange of serines/threonines at phosphorylated sites into phosphomimetic aspartates reduces the $\mathrm{Ca}^{2+}$ affinity of the recombinant $\mathrm{C}_{2} \mathrm{~F}$ domain 10 -fold, and increases the $\mathrm{Ca}^{2+}$ affinity of the $\mathrm{C}_{2} \mathrm{C}$ domain. Concordantly, we show that phosphorylation of otoferlin and/or its interaction partners are enhanced upon hair cell depolarization and blocked by pharmacological CaMKII inhibition. We therefore propose that otoferlin activity is regulated by CaMKII\& in IHCs.

Keywords: $\mathrm{C}_{2}$ domains, hair cell, synaptic transmission, $\mathrm{Ca}^{2+}$ affinity, phosphorylation, CaMKII

Abbreviations: CaMKIII, $\mathrm{Ca}^{2+} /$ calmodulin-dependent serine/threonine kinase II delta; IHC, inner hair cell; IP, Immunoprecipitation; LC-MS/MS, liquid chromatography tandem mass spectrometry; MST, MicroScale Thermophoresis; $\mathrm{OC}$, organ of Corti; PLA, proximity ligation assay. 


\section{INTRODUCTION}

Otoferlin is a $230 \mathrm{kDa}$, tail-anchored membrane protein, containing at least six $\mathrm{C}_{2}$ domains implicated in $\mathrm{Ca}^{2+}$, phospholipid, and protein binding (Yasunaga et al., 1999; Johnson and Chapman, 2010; Pangršič et al., 2012). Dysfunction of otoferlin underlies DFNB9, a recessive and non-syndromic form of prelingual deafness in humans characterized by impaired synaptic transmission from IHCs (Yasunaga et al., 1999). Unique to IHC ribbon synapses, otoferlin is hypothesized to operate as a $\mathrm{Ca}^{2+}$-sensor in synaptic vesicle fusion (Roux et al., 2006), and it was shown to be involved in vesicle replenishment, vesicle reformation from bulk endosomes, active zone clearance, and clathrin-mediated endocytosis (Pangršič et al., 2010; Duncker et al., 2013; Jung et al., 2015; Strenzke et al., 2016). To date, several protein interaction partners of otoferlin have been reported including myosin VI, Rab8b, SNARE proteins, Cav1.3 $\mathrm{Ca}^{2+}$ channel, Ergic2 and AP-2 (Roux et al., 2006; Heidrych et al., 2008, 2009; Ramakrishnan et al., 2009; Roux et al., 2009; Zak et al., 2012; Duncker et al., 2013; Jung et al., 2015). However, the physiological effects of many of these interactions remain only partially understood. In this study, we aimed to identify new otoferlin interaction partners and to address a potential role of these interactions in IHC synaptic function.

Neurotransmitter release from IHCs is extraordinary in several respects. Firstly, it is precisely coupled to the cycle of auditory sine waves generating graded receptor potentials in IHCs up to $3 \mathrm{kHz}$ in rodents (Palmer and Russell, 1986). Secondly, release is largely indefatigable with a sustained vesicle fusion rate of up to 2300 vesicles per second per active zone (Strenzke et al., 2016). Thirdly, exocytosis elicits large EPSCs to reliably trigger postsynaptic spikes (Glowatzki and Fuchs, 2002; Rutherford et al., 2012), and fourthly, exocytosis from IHCs does not require neuronal SNARE proteins (Nouvian et al., 2011). Remarkably, the 10-20 ribbon synapses in each IHC respond differently to the same graded depolarization, a process required to encode different sound intensities, the molecular mechanisms of which are only beginning to be understood (Merchan-Perez and Liberman, 1996; Taberner and Liberman, 2005; Frank et al., 2009; Meyer et al., 2009; Hickman et al., 2015; Ohn et al., 2016; Reijntjes and Pyott, 2016).

Presynaptic activity was reported to be regulated in many synapses of the central nervous system and in sensory systems, e.g., by phosphorylation of presynaptic proteins, thereby leading to adaptation (or facilitation) to constant stimuli. The auditory system does not grossly adapt, at least not to mild or moderate sound stimuli. In contrast, exposure to noise can cause both temporary and permanent threshold shifts, depending on stimulus levels and duration (Kujawa and Liberman, 2009). While a number of mechanisms have been suggested to underlie a temporary threshold shift, adaptation of the presynaptic machinery has not been studied to date.

Here, we studied the interaction of the presynaptic IHC protein otoferlin with CaMKII $\delta$, the induction of otoferlin phosphorylation and the effects of phosphorylation on $\mathrm{Ca}^{2+}$ binding. Our data indicate that the functions of otoferlin in exocytosis, vesicle replenishment and endocytosis might be regulated during strong IHC stimulation.

\section{MATERIALS AND METHODS}

\section{Animal Welfare}

Animal handling complied with national animal care guidelines. For rats and chicken, handling was approved by the Administrative Panel on Laboratory Animal Care (APLAC) of Stanford University and accredited by the Association for the Assessment and Accreditation of Laboratory Animal Care (\#A3213-01). For mice, handling was approved by the University of Göttingen Board for animal welfare and the animal welfare office of the state of Lower Saxony, Germany.

\section{Co-immunoprecipitation of Otoferlin from Chicken Utricle Hair Cells}

Utricles were dissected from embryonic day 18 (E18) old chicken. Otoconial membranes and spiral ganglion nerve fibers were removed and utricles were collected into chilled lysis buffer (50 mM Tris- $\mathrm{HCl}, 140 \mathrm{mM} \mathrm{NaCl}, 5 \%$ Glycerol, $250 \mathrm{mM}$ sucrose, protease inhibitors (Roche, EDTA-free), $\mathrm{pH}$ 7.4). The tissue was homogenized by triturating six times through a 26 gauge needle, followed by centrifugation for $5 \mathrm{~min}$ at $600 \times \mathrm{g}$ at $4^{\circ} \mathrm{C}$ (Eppendorf tabletop Centrifuge $5417 \mathrm{C}$ ). The supernatant was subsequently centrifuged at $100000 \times g$ for $30 \mathrm{~min}$ at $4^{\circ} \mathrm{C}$ to pellet membranes (Beckmann, TL-100 Ultracentrifuge). The pellet was resuspended in solubilization buffer (20 mM Tris-HCl, $10 \%$ glycerol, $140 \mathrm{mM} \mathrm{NaCl}, 1 \%$ octyl$\beta$-D-glucopyranoside, protease inhibitors, $\mathrm{pH} 7.4$ ) for $1 \mathrm{~h}$ on ice. After solubilization, samples were centrifuged for $20 \mathrm{~min}$ at $55000 \times g$ at $4^{\circ} \mathrm{C}$ (Beckmann, TL-100 Ultracentrifuge). Supernatants were incubated with $25 \mu \mathrm{L}$ Dynabeads (Life Technologies, Dynabeads M-270 Epoxy) conditioned with either $5 \mu \mathrm{g}$ chicken HCS-1 antibody (mouse, monoclonal; Goodyear et al., 2010) or $5 \mu \mathrm{g}$ control antibody, TLA (tip-link antigen, mouse monoclonal; Goodyear and Richardson, 2003), according to the manufacturer's protocol. Immunoprecipitation of otoferlin was performed for $2 \mathrm{~h}$ at $4^{\circ} \mathrm{C}$. Beads were washed three times for 15 min at $4^{\circ} \mathrm{C}$ with solubilization buffer containing $0.1 \%$ octyl$\beta$-D-glucopyranoside. Beads were then boiled for $5 \mathrm{~min}$ at $95^{\circ} \mathrm{C}$ in Laemmli sample buffer (BioRad), loaded onto a $4-20 \%$ SDS PAGE (BioRad) and proteins were allowed to run $1 \mathrm{~cm}$ into the separation gel and visualized with Coomassie brilliant blue staining (BioRad).

\section{Mass Spectrometric Analysis of Immunoprecipitated Otoferlin and Interacting Proteins}

Gel bands were excised and digested in-gel using trypsin (Promega) as previously described (Shevchenko et al., 2006). Dried peptides were reconstituted in $0.1 \%$ formic acid, $2 \%$ acetonitrile and $97.9 \%$ water. Peptides were loaded onto a selfpacked C18 reverse phase column with an ID of $100 \mu \mathrm{M}$ and 
$15 \mathrm{~cm}$ in length. Over the course of all LC-MS/MS experiments, two LCs were used: a nanoAcquity UPLC (Waters) and a nano2D LC (Eksigent, AB Sciex), with flow rates of $300 \mathrm{~nL} / \mathrm{min}$ and mobile phase A consisting of $0.585 \%$ ( $\mathrm{vol} / \mathrm{vol}$ ) acetic acid in water and mobile phase B of $0.585 \%(\mathrm{vol} / \mathrm{vol})$ acetic acid and $2 \%(\mathrm{vol} / \mathrm{vol})$ water in acetonitrile. The mass spectrometer (LTQ Orbitrap Velos, Thermo Fisher) utilized data-dependent acquisition in which the top 12 most intense precursor ions were selected for fragmentation. The raw data were converted to mzXML format and searched against the UniProt Gallus gallus database using Sequest on a sorcerer platform (SageN). Search parameters included tryptic specificity, allowing for a maximum of two miscleavages with static modification of propionamide (cysteine) and variable modifications of oxidation (methionine), phosphorylation (serine, threonine, tyrosine) and alkylation (lysine). The precursor mass tolerance was $20 \mathrm{ppm}$, and the data was further filtered using the Scaffold software (Proteome Software). In the case of identified interacting partners we stringently required at least 4 unique peptides with a $95 \%$ peptide threshold and $99.9 \%$ protein probability threshold, thereby effectively filtering out all non-specific contaminants.

\section{Real-time PCR Experiments}

PCRs on a few IHCs were performed essentially as described (Kerr et al., 2008; Reisinger et al., 2011). Organs of Corti (OCs) from P14 mice were dissected in HEPES-Hanks solution (5.36 $\mathrm{mM} \mathrm{KCl}, 141.7 \mathrm{mM} \mathrm{NaCl}, 1 \mathrm{mM} \mathrm{MgCl}_{2}, 0.5 \mathrm{mM} \mathrm{MgSO}_{4}$, $10 \mathrm{mM}$ Na-HEPES, $6.84 \mathrm{mM}$ L-glutamine, $5.55 \mathrm{mM}$ D-glucose, pH 7.2) and perfused with modified Ringer's solution thereafter (113 mM NaCl, $35 \mathrm{mM}$ TEA-Cl, $2.8 \mathrm{mM} \mathrm{KCl}, 2 \mathrm{mM} \mathrm{CaCl}_{2}$, $1 \mathrm{mM} \mathrm{MgCl} 2,10 \mathrm{mM}$ Na-HEPES, $1 \mathrm{mM} \mathrm{CsCl}, 11.1 \mathrm{mM}$ D-glucose; $\mathrm{pH}$ adjusted to 7.2 , osmolarity approximately 300 mOsm). Outer hair cells and supporting cells were removed with glass capillaries in a patch-clamp setup. Once inner phalangeal cells were removed, 3-5 IHCs were collected in one patch clamp glass capillary filled with $8 \mu \mathrm{L} \mathrm{KCl}$ solution $(140 \mathrm{mM} \mathrm{KCl}, 5 \mathrm{mM}$ K-HEPES, 5 mM EGTA, 3 mM MgCl 2 , pH 7.3). Before and after collecting cells, bath controls were taken by lowering the patch pipette close to the tissue and removing overpressure for 5-10 s. The content of each capillary was expelled into a reaction tube containing buffer for reverse transcription $[2.5 \mu \mathrm{L}$ first strand buffer, $0.6 \mu \mathrm{L}$ Oligo $(\mathrm{dT})_{20}$ primer $(50 \mu \mathrm{M}), 0.5 \mu \mathrm{L}$ Random hexamers $(50 \mathrm{ng} / \mu \mathrm{L}), 0.7 \mu \mathrm{L}$ dNTP $\operatorname{mix}(10 \mathrm{mM}$ each $), 1.4 \mu \mathrm{L}$ $\operatorname{DTT}(0.1 \mathrm{M})$ and $0.8 \mu \mathrm{L}$ Ribonuclease Inhibitor $(40 \mathrm{U} / \mu \mathrm{L})]$. The reaction was started by adding $0.5 \mu \mathrm{L}$ SuperScript ${ }^{\circledR}$ IV Reverse Transcriptase (100 units; Thermo Fisher Scientific) and incubated for $10 \mathrm{~min}$ at room temperature (RT), followed by $20 \mathrm{~min}$ at $37^{\circ} \mathrm{C}$ and $2 \mathrm{~h}$ at $42^{\circ} \mathrm{C}$. The resulting cDNA was precipitated over night at $-20^{\circ} \mathrm{C}$ in $70 \% \mathrm{EtOH}$ and $1 \mu \mathrm{L}$ glycogen (Ambion), washed with $70 \% \mathrm{EtOH}$, dried and resuspended in $25 \mu \mathrm{L} \mathrm{H}_{2} \mathrm{O}$. The cDNA solution from each sample was split into six PCR reactions. cDNA quality was assessed with TaqMan assays for bassoon (Mm00464451_m1; Applied Biosystems) and TATA-binding protein (Mm00446973_m1; Applied Biosystems). To test for CaMKII isoforms in a SYBR green assay, we designed intron-overspanning amplicons targeting all splice variants of the four CaMKII genes (in Mus musculus) described as reference sequences in NCBI databases. Amplification efficiency of the assays was determined with standard curve assays, resulting in 95.7-96.9\% efficiencies for all transcripts. We used the following oligonucleotides: CaMKII $\alpha$ : $5^{\prime}$-GAAGATGTGCGACCCTGGAA-3 ${ }^{\prime}$ and $5^{\prime}$-TGA TGCGGATATAGGCGATG-3' (400 nM each), CaMKIIß: 5'-AC AAACAGCACCAAAAACAGCT- $3^{\prime}$ and $5^{\prime}$-GAGCTGCTCTGT GGTCTTGA-3' (300 nM each), CaMKII $\gamma: 5^{\prime}$-TTACGCAAATT CAACGCCCG-3' and 5'-GACACCGCCATCTGACTTCT-3' (400 nM each), CaMKII $\delta: 5^{\prime}$-CGTCTCTTGAAGCACCCCAA$3^{\prime}$ and $5^{\prime}$-AAACAGTTCGCCACCAGTCA-3' (300 nM each). Mouse brain CDNA was used as positive control. Amplification with 2x TaqMan universal PCR Mastermix (Applied Biosystems) or $2 x$ Power SYBR green Mastermix (Applied Biosystems) was conducted in an Applied Biosystems 7500 Real Time PCR system using default PCR parameters. Dissociation curve assays revealed the melting temperatures to control for amplicon specificity. In addition, we assayed the size of all amplicons with gel electrophoresis on $2 \%$ agarose gels with EtBr staining. We analyzed only those IHC samples for CaMKII expression where both bassoon and TATA-binding protein transcripts were detected.

\section{Immunohistochemistry}

Sprague Dawley rats at P9 to P11 or P14 C57B16J mice of either gender were decapitated and cochleae were dissected in chilled Hank's balanced salt solution (HBSS, HyClone). OCs were fixed in chilled 3\% paraformaldehyde for $25 \mathrm{~min}$, permeabilized with $0.5 \%$ Triton X-100 in PBS for 30 min and incubated in blocking solution ( $1 \% \mathrm{BSA}$ and $0.1 \%$ Triton X-100 in PBS) for $1 \mathrm{~h}$ at RT. Antibodies to otoferlin (mouse, 1:400; Abcam, ab53233), myosin VI (rabbit, 1:400; Sigma, M5187), parvalbumin (1:2000, raised against bullfrog parvalbumin-3, in mice it recognizes oncomodulin and parvalbumin; Heller et al., 2002), CaMKII $\alpha$ (rabbit, 1:300; Sigma-Aldrich, C6974), CaMKII $\beta$ (rabbit, 1:300; Abcam, ab34703), CaMKII $\gamma$ (rabbit, 1:300; Acris, AP13886PU-N), CaMKII (rabbit, 1:300; Genetex, GTX111401), pan-CaMKII (rabbit, 1:300; Abcam, ab52476), PSD95 (mouse, 1:500, Abcam, ab2723), Ctbp2 (goat, 1:200, Santa Cruz, sc-5966) and phosphoserine (rabbit, 1:300; Abcam, 9332), were diluted in blocking solution and incubated on OCs overnight at $4^{\circ} \mathrm{C}$. Alexa fluor $488,546,568$, and 633-conjugated secondary antibodies (1:200-1:600; Thermo Fisher Scientific) were used. The tissue was mounted with Aqua Poly/Mount (Polysciences) and for Figures 3, 4, and 10 imaged using an AxioImager/LSM 5 Exciter confocal microscope (Zeiss). Images in Figure 1 were acquired using a Leica SP5 confocal microscope with $63 x$ glycerol objective (NA 1.456).

\section{Proximity Ligation Assay}

P9-P11 rat OCs were dissected in chilled external solution (10 mM HEPES, $2 \mathrm{mM} \mathrm{MgCl}, 2 \mathrm{mM} \mathrm{CaCl}_{2}, 2 \mathrm{mM} \mathrm{KCl}$, $145 \mathrm{mM} \mathrm{NaCl}, 6 \mathrm{mM}$ D-glucose, $2 \mathrm{mM}$ ascorbate, $2 \mathrm{mM}$ pyruvate, $2 \mathrm{mM}$ creatine, $\mathrm{pH} 7.4$ ) and fixed in chilled $3 \%$ paraformaldehyde for 25 min. For hair cell stimulation, acutely dissected OCs were transferred into prewarmed high $\mathrm{K}^{+}$external 
Appendix | 149
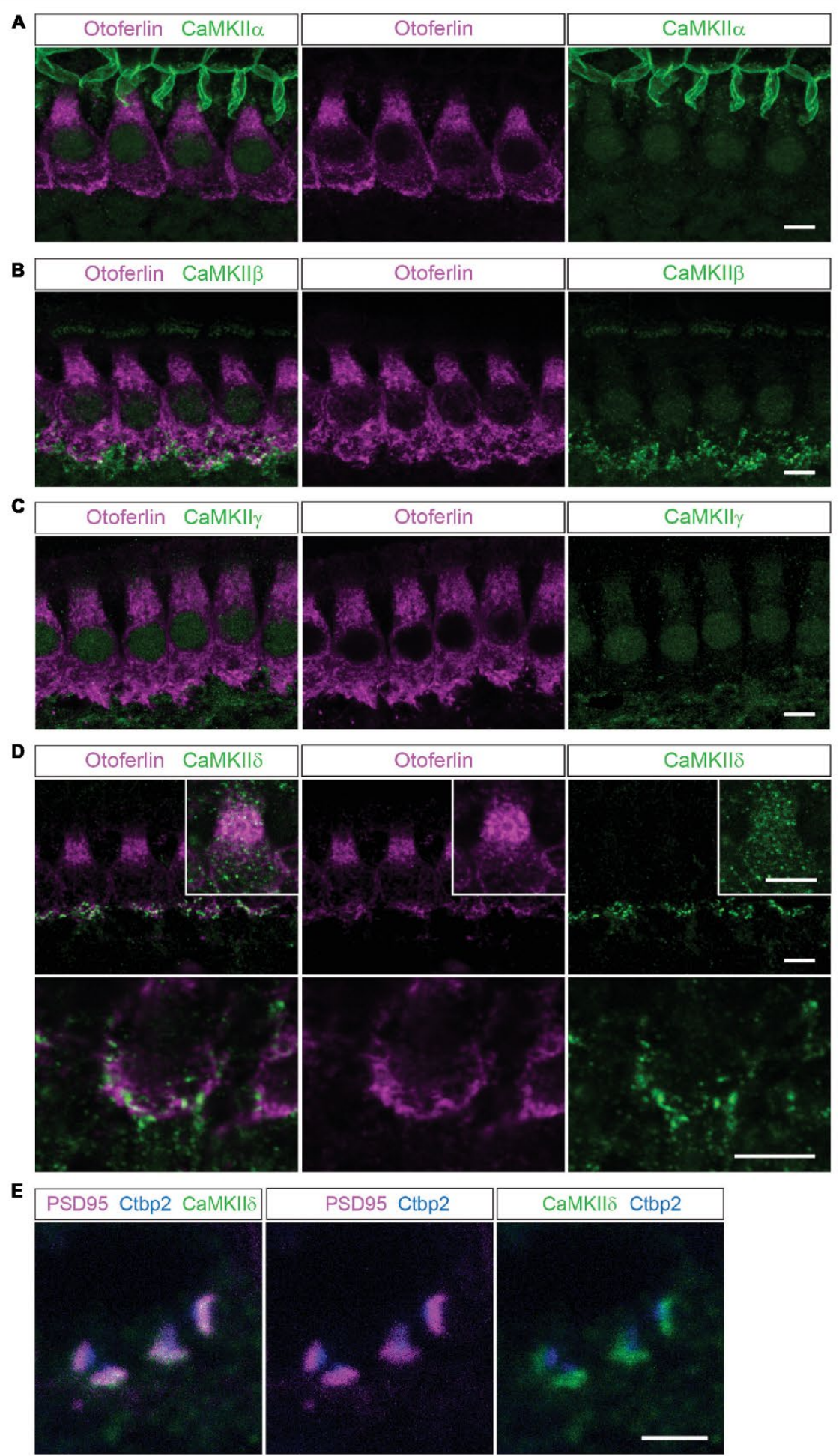

FIGURE 1 | Localization of the different CaMKIls in the organ of Corti. (A) Single optical sections acquired by confocal microscopy display immunolabelling of CaMKIla (green) in P14 mouse IHCs, co-labeled for otoferlin (magenta). Here, no definite expression in IHCs could be detected. (B-E), CaMKIIB, $\gamma$ and $\delta$ are present in structures outside the IHCs, possibly in efferent and/or afferent synaptic boutons. (D), CaMKIII can additionally be detected in the cytoplasm of IHCs, visualized in insets with enhanced CaMKII $\delta$ fluorescence. Scale bars in (A-D), $5 \mu \mathrm{m}$. (E), co-labeling against the ribbon marker Ctbp2 and the postsynaptic protein PSD95

indicates a localization of CaMKIll at the postsynaptic site of afferent synaptic boutons; scale bar $2 \mu \mathrm{m}$. 
solution ( $\mathrm{KCl}$ increased to $40 \mathrm{mM}, \mathrm{NaCl}$ reduced to $35 \mathrm{mM}$ ) and incubated for $15 \mathrm{~min}$ at $37^{\circ} \mathrm{C}$ and $5 \% \mathrm{CO}_{2}$ before fixation. To pharmacologically inhibit CaMKII, OCs were incubated for $10 \mathrm{~min}$ in prewarmed external solution supplemented with $50 \mu \mathrm{M}$ of selective inhibitor KN-93 (Cayman Chemical) followed by stimulation with high $\mathrm{K}^{+}$external solution $+\mathrm{KN}-93$ and fixation. A proximity ligation assay (PLA) (Duolink, Sigma) was performed with mouse otoferlin (1:500; Abcam, ab53233) antibody in combination with rabbit myosin VI (1:500; Sigma, M5187), rabbit parvalbumin (1:2000; Heller et al., 2002), rabbit CaMKIIS (1:300; Genetex, GTX111401), rabbit phosphoserine (1:300; Abcam, 9332), and rabbit pan-CaMKII (1:300; Abcam, ab52476). The manufacturer's protocol was applied with the following modifications: Fixed OCs were semipermeabilized in $0.5 \%$ Triton X-100 at RT for $30 \mathrm{~min}$ and subsequently blocked with Blocking buffer (Duolink, Sigma) for $2 \mathrm{~h}$ at RT. Primary antibodies were diluted in antibody diluent (Duolink, Sigma) and incubated overnight at $4^{\circ} \mathrm{C}$. The tissue was then washed four times with gentle shaking in $2 \mathrm{~mL}$ Buffer A (Duolink, Sigma). PLA probes (anti mouse MINUS and anti-rabbit PLUS; Duolink, Sigma) were diluted 1:6 in $30 \mu \mathrm{L}$ antibody diluent and incubated for $1 \mathrm{~h}$ at $37^{\circ} \mathrm{C}$ and $5 \% \mathrm{CO}_{2}$. Tissue was washed four times with $2 \mathrm{~mL}$ Buffer $\mathrm{A}$ and gentle shaking, followed by incubation with $30 \mu \mathrm{L}$ ligation mix for $30 \mathrm{~min}$ at $37^{\circ} \mathrm{C}$ and $5 \% \mathrm{CO}_{2}$. After three washes for $10 \mathrm{~min}$ in $2 \mathrm{~mL}$ Buffer $A$ and gentle shaking, $30 \mu \mathrm{L}$ DNA amplification mix was added and incubated for $100 \mathrm{~min}$ at $37^{\circ} \mathrm{C}$ and $5 \% \mathrm{CO}_{2}$. After amplification, the tissue was washed twice for $10 \mathrm{~min}$ in $1 \mathrm{x}$ Buffer B (Duolink, Sigma) followed by two times washing in $2 \mathrm{~mL}$ $0.01 \mathrm{x}$ Buffer B. To visualize hair cells, tissue was subsequently stained with Alexa Phalloidin 488 (Invitrogen) (1:200 in 0.01x Buffer B) for $15 \mathrm{~min}$ at RT, followed by two $10 \mathrm{~min}$ washing steps in $2 \mathrm{~mL} \mathrm{0.01x} \mathrm{Buffer} \mathrm{B.} \mathrm{For} \mathrm{counterstainings} \mathrm{of} \mathrm{synaptic}$ ribbons, anti-Ctbp2 antibody (goat, 1:200, Santa Cruz, sc-5966) was incubated together with primary antibodies to otoferlin and phosphoserine, and secondary anti-goat antibodies were coincubated with PLA probes. For imaging, tissue was mounted with DAPI containing Duolink In Situ Mounting media. Images were taken with a LSM700 confocal microscope (Zeiss) with Zen software (Zeiss).

\section{Quantification of PLA Signals and Statistical Analysis}

To quantify PLA signal intensities in confocal images, IHCs were outlined manually using Image J software and pixel intensities of the fluorescent PLA signals were determined. For each experimental condition three independent experiments were performed and a total of $30 \mathrm{IHCs}$ were analyzed. The mean pixel intensity per IHC was calculated for each condition and compared. A two-tailed $t$-test was applied to assess a statistical significance of the changes in PLA signal pixel intensities amid the different experimental conditions.

\section{In Vitro Pull-Down Assays}

Two mouse otoferlin fragments $\left(\mathrm{C}_{2} \mathrm{ABC}\right.$ : aa $1-632,70 \mathrm{kDa}$ and $\mathrm{C}_{2}$ DEF: aa $933-1920,114 \mathrm{kDa}$ ) were PCR amplified from cDNA encoding full-length mouse otoferlin (NM_001100395) with a C-terminal HA-tag using the following primer pairs: $\mathrm{C}_{2} \mathrm{ABC}-\mathrm{HA}$ (5'-GAATTCACCATGGCCCTGATTGTTCACCT-3', 5'-GCGG CCGCCTAAGCGTAATCTGGAACATCGTATGGGTACATGG TTCCTCCTGTGCAGCTCTCCGAGACAG-3'); $\mathrm{C}_{2}$ DEF (5'-GA ATTCACCATGAGCAAGCAG-CGAAAGGACTTC-3', 5'-GCG GCCGCCTAAGCGTAATCTGGAACATCGTATG-GGTACAT GGTTCCTCCGCGAGCCAGGCCCACAGGG-3'). Fragments were subcloned into the $\mathrm{pCl}$ mammalian expression vector (Promega) via NotI and EcoRI. Full-length CaMKII8 (NM_00 1025438.1) was amplified from mouse postnatal day 6 (P6) cochlea cDNA (5'-GAATTCACCATGGCTTCGACCACCACCT G-3', 5'-GGTACCCCGATGTTTTG-CCACAAAGAGG-3') and subcloned as $\mathrm{N}$-terminal fusion construct into the pmCherryN1 mammalian expression vector (Clontech) via EcoRI and KpnI. For co-immunoprecipitation (co-IP) of otoferlin, HEK293 cells were grown in a $10 \mathrm{~cm}$ culture dish to $80 \%$ confluency and transiently co-transfected with HA-tagged $\mathrm{C}_{2} \mathrm{ABC}$, HA-tagged $\mathrm{C}_{2} \mathrm{DEF}$ and mcherry-tagged CaMKII $\delta$. $40 \mathrm{~h}$ post-transfection, cells were collected in TBS buffer (25 mM Tris- $\mathrm{HCl}, 150 \mathrm{mM} \mathrm{NaCl}$, protease inhibitors (Roche, complete, EDTA free), $\mathrm{pH}$ 7.4), lysed by triturating 5 times through a $26^{1 / 2}$ gauge needle, and centrifuged for $5 \mathrm{~min}$ at $4^{\circ} \mathrm{C}$ at $500 \times \mathrm{g}$ to remove debris. For co-IP of CaMKII $\delta$-mcherry with otoferlin $\mathrm{C}_{2} \mathrm{ABC}-\mathrm{HA}$ and/or $\mathrm{C}_{2}$ DEF-HA, the lysate was mixed with anti-HA agarose slurry (35 $\mu \mathrm{g}$ anti-HA antibody, Pierce HA Tag IP/Co-IP Kit, Thermo Scientific) and incubated with gentle endover-end mixing for $2 \mathrm{~h}$ at $4^{\circ} \mathrm{C}$. Agarose was washed four times with TBS-T buffer $(25 \mathrm{mM}$ Tris- $\mathrm{HCl}, 150 \mathrm{mM} \mathrm{NaCl}$, $0.05 \%$ Tween, $\mathrm{pH} 7.4$ ) before boiling for $5 \mathrm{~min}$ at $95^{\circ} \mathrm{C}$ in Laemmli sample buffer and applied on a $4-20 \%$ SDS PAGE gel (BioRad). Protein complexes were analyzed by immunoblots using a Trans Blot semi-dry transfer cell (BioRad) and polyclonal anti-HA antibodies (1:1000; Rockland, 600401-384) and monoclonal anti-RFP antibodies (1:20000; Rockland, 200-301-379). Secondary anti-rabbit Dylight680 and anti-mouse Dylight800 antibodies (1:10000; Rockland, 611-144-003 and 610-145-003) were incubated for $1 \mathrm{~h}$ at RT, and after washing the blots for three times with TBS-T buffer, fluorescent signals were detected using a Li-Cor Odyssey system.

\section{Recombinant Expression of Otoferlin Fragments}

Two soluble mouse otoferlin domains comprising either the first three $\mathrm{C}_{2}$ domains $\left(\mathrm{C}_{2} \mathrm{ABC}\right)$, or the last three $\mathrm{C}_{2}$ domains $\left(\mathrm{C}_{2} \mathrm{DEF}\right)$ were heterologously expressed in Escherichia coli (E. coli) SoluBL21 (DE3). The $\mathrm{C}_{2} \mathrm{ABC}$ fragment (aa 1-616, $70 \mathrm{kDa}$; NP_001093865) was PCR amplified (5'-AGCGGCTCT TCAATG-ATGGCCCTGATTGTTCACCT-3', 5'-AGCGGCTC TTCTCCC-CTCCGAGACAGGCGTGGC-3') and subcloned with a C-terminal hexahistidine-tag into the bacterial expression vector pPSG-IBA33 (Iba Lifesciences) and expressed at $30^{\circ} \mathrm{C}$. After induction with IPTG, the temperature was changed to $16^{\circ} \mathrm{C}$ and the culture was harvested $16-20 \mathrm{~h}$ post-induction. 
The cells were collected in lysis buffer $(70 \mathrm{mM}$ HEPES pH 7.4, $300 \mathrm{mM} \mathrm{NaCl}, 10 \mathrm{mM}$ imidazole) and lysed by fluidizing (microfluidizer S, Microfluidics, Westwood, MA, United States). After centrifugation at $20000 \mathrm{rpm}$ (JA-20 fixed angle rotor, Beckmann Coulter) for $45 \mathrm{~min}$ at $4^{\circ} \mathrm{C}$, the supernatant was loaded onto a Ni-NTA-column (GE Healthcare). After washing, the recombinant proteins were eluted by gradient elution with imidazole containing buffer $(70 \mathrm{mM}$ HEPES, $300 \mathrm{mM} \mathrm{NaCl}$, $500 \mathrm{mM}$ imidazole, pH 7.4). For buffer exchange to $10 \mathrm{mM}$ HEPES, $300 \mathrm{mM} \mathrm{NaCl}$, pH 7.4, the proteins were further applied on a size exclusion chromatography column (HiPrep 16/60 Sephacryl S-200 HR, GE Healthcare). During all purification steps the temperature was kept at $4^{\circ} \mathrm{C}$.

C 2 DEF (aa 908-1932, $118 \mathrm{kDa}$; NP_001093865) was PCR amplified (5'-GAGAGGATCCAAGCTGGAGCTCTACCTGTG$3^{\prime}, \quad 5^{\prime}$-GAGAGAATTCTAATCA-GGTTCATTGCGAGCCAG$\left.3^{\prime}\right)$ and subcloned with a hexahistidine tag into the bacterial expression vector pET28a. Expression took place for $60 \mathrm{~h}$ at $16^{\circ} \mathrm{C}$ using an autoinduction system (Studier, 2005).

The $\mathrm{C}_{2} \mathrm{DEF}$ was purified from inclusion bodies by resuspending the cell pellet in lysis buffer. After centrifugation for $45 \mathrm{~min}$ at $4^{\circ} \mathrm{C}$ and $20000 \mathrm{rpm}$ (JA-20 fixed angle rotor, Beckmann Coulter), the supernatant was discarded and the pellet was washed three times with PBS buffer $\left(4 \mathrm{mM} \mathrm{KH}_{2} \mathrm{PO}_{4}\right.$, $16 \mathrm{mM} \mathrm{Na}_{2} \mathrm{HPO}_{4}, 115 \mathrm{mM} \mathrm{NaCl}, \mathrm{pH} 7.4$ ), containing $1 \%$ Triton in the first washing step. The pellets were frozen overnight at $-20^{\circ} \mathrm{C}$ and subsequently dissolved in Urea buffer $(70 \mathrm{mM}$ HEPES, $300 \mathrm{mM} \mathrm{NaCl}, 10 \mathrm{mM}$ imidazole, $8 \mathrm{M}$ urea, $\mathrm{pH}$ 7.4). After centrifugation, the supernatant was loaded onto a Ni-NTAcolumn (GE Healthcare) and recombinant proteins were eluted with an imidazole gradient.

\section{In Vitro Phosphorylation Assay}

For in vitro phosphorylation of recombinant otoferlin, $21.5 \mathrm{pmol}$ recombinant CaMKII $\delta$ (Life Technologies) was incubated together with equimolar amounts of recombinant otoferlin $\mathrm{C}_{2} \mathrm{ABC}$ and $\mathrm{C}_{2} \mathrm{DEF}$ domains (1:1:1) in $30 \mu \mathrm{L}$ assay buffer (10 mM HEPES, $10 \mathrm{mM} \mathrm{MgCl} 2,10 \mu \mathrm{g} / \mathrm{mL}$ calmodulin, $0.5 \mathrm{mM}$ $\mathrm{CaCl}_{2}, 5 \mathrm{mM}$ DTT, $100 \mu \mathrm{M}$ ATP, $\mathrm{pH}$ 7.5) for $5 \mathrm{~min}$ at $30^{\circ} \mathrm{C}$. The reaction was inactivated by adding Laemmli-buffer and subsequent incubation at $95^{\circ} \mathrm{C}$ for $5 \mathrm{~min}$. For control experiments, otoferlin $\mathrm{C}_{2} \mathrm{ABC}$ and $\mathrm{C}_{2} \mathrm{DEF}$ were incubated in assay buffer in the absence of CaMKII $\delta$.

\section{Mass-Spectrometric Analysis of Otoferlin Phosphorylation Sites}

Gel bands corresponding to recombinant mouse otoferlin fragments, $\mathrm{C}_{2} \mathrm{ABC}(70 \mathrm{kDa})$ and $\mathrm{C}_{2} \mathrm{DEF}(118 \mathrm{kDa})$, were excised from the Coomassie gel after in vitro phosphorylation and prepared for LC-MS/MS analysis as described in above. During data acquisition the mass spectrometer was set to perform ion-trap MS/MS and high energy collision-induced dissociation (HCD) MS/MS on the same precursor masses to provide more complete fragmentation data and to increase the probability of correctly localizing the site of phosphorylation. All suggested phosphorylation sites were manually validated by interrogation of the fragment ion spectra, where neutral loss of phosphoric acid $\left(\mathrm{H}_{3} \mathrm{PO}_{4}\right)$ was observed as well as site localization of the phosphor-group by corresponding $b$ or $y$ ions.

\section{Expression and Phosphomimetic Mutagenesis of $\mathrm{C}_{2} \mathrm{C}$ and $\mathrm{C}_{2} \mathrm{~F}$ Domains}

The protein fragments of otoferlin used for $\mathrm{Ca}^{2+}$ binding assays $-\mathrm{C}_{2} \mathrm{C}$ (aa 410-616 in pGEX-6P-3, NP_001263649) and $\mathrm{C}_{2} \mathrm{~F}$ (aa 1695-1934 in pGEX-6P-3, NP_001263649.1) were expressed in E. coli Rosetta 2 (DE3) cells using the autoinduction system (Studier, 2005; Meese, 2015). The harvested cells were lysed in $75 \mathrm{mM}$ HEPES pH $7.4,300 \mathrm{mM} \mathrm{NaCl}$ using the microfluidizer $S$ (Microfluidics, Westwood, MA, United States). The obtained supernatant after centrifugation was loaded onto $5 \mathrm{~mL}$ GST Trap columns (GE Healthcare). Using a glutathion containing buffer (75 mM HEPES pH 7.4, $300 \mathrm{mM} \mathrm{NaCl}, 25 \mathrm{mM}$ reduced glutathion) the protein was eluted from the column and incubated with PreScission protease for $14 \mathrm{~h}$ at $4^{\circ} \mathrm{C}$ to cleave off the GST-tag. In the next step a size exclusion chromatography (S200 16/60, GE Healthcare) was performed (10 mM HEPES pH 7.4, $150 \mathrm{mM}$ $\mathrm{NaCl}$ ) followed by a GST trap column to separate the $\mathrm{C}_{2}$ domain from the GST-tag. The protein solution was incubated with Chelex (Biorad) for $1 \mathrm{~h}$ at $4^{\circ} \mathrm{C}$, concentrated and stored at $-80^{\circ} \mathrm{C}$.

In order to mimic phosphorylation sites, we replaced phosphorylated serine/threonine residues with aspartate residues. The cDNA for the "C2F-pm" fragment (S1783D, S1814D, T1866D) was newly synthesized by GeneArt (Life Technologies). For the "C2C-pm" fragment a mutation (T434D) was inserted using a "QuikChange" site directed mutagenesis protocol (Agilent Technologies). The expression and purification procedure was the same as for the wild type proteins.

\section{$\mathrm{Ca}^{2+}$ Affinity Measurement by MicroScale Thermophoresis (MST)}

For MicroScale Thermophoresis (MST) the NT.LabelFree instrument (NanoTemper Technologies GmbH, Munich, Germany) was used. All solutions were treated with Chelex (Biorad) to remove residual $\mathrm{Ca}^{2+} . \mathrm{CaCl}_{2}(1 \mathrm{M}$ solution, Fluka) was diluted in size exclusion buffer (10 mM HEPES, $150 \mathrm{mM}$ $\mathrm{NaCl}, 0.05 \%$ Tween, $\mathrm{pH} 7.4$ ) and a series of 16 dilutions (1:2) was prepared and mixed with protein, resulting in ligand concentrations ranging from $0.6 \mu \mathrm{M}$ to $200 \mathrm{mM}$. Proteins were used in concentrations of $1-4 \mu \mathrm{M}$. For the negative control 50 or $500 \mathrm{mM}$ EDTA was added to the reaction mixture. The samples were filled into NT.LabelFree Standard Treated Capillaries (NanoTemper Technologies $\mathrm{GmbH}$ ). The measurement took place at $22^{\circ} \mathrm{C}$ with laser off/on/off times of 5,39 , or $5 \mathrm{~s}$. The instrument parameters were adjusted to $5 \%$ LED power and 20\% MST power. The data presented here are from three technical replicates done in the same day; the whole experiment was repeated at least three times confirming the results. We analyzed the temperature jump (fluorescence change during the first second of IR radiation exposure) for each sample. 
Fluorescence change during temperature jump was plotted against ligand concentration and curves were fitted with the Hill Fit:

$$
f(c)=\text { unbound }+(\text { bound }- \text { unbound }) /\left(1+(E C 50 / c)^{\wedge} n\right)
$$

in IGOR (Wavemetrics).

\section{RESULTS}

\section{A Pull-Down from Chicken Utricle Reveals CaMKIII as a Novel Otoferlin Interaction Partner}

In order to identify interaction partners of otoferlin, we used E18 vestibular maculae of the chicken utricle, each containing more than 20000 hair cells for affinity purification of otoferlin. Most vestibular hair cells are functional at this late embryonic age (Goodyear et al., 1999), and utricles can be dissected relatively quickly in larger numbers (Herget et al., 2013). Membrane proteins of 60 avian utricular maculae were solubilized with octyl- $\beta$-D-glucopyranoside (Kim et al., 2004) and otoferlin and its potential binding partners were purified using the monoclonal anti-chicken otoferlin antibody HCS-1 (Goodyear et al., 2010), immobilized to magnetic dynabeads. The HCS-1 antibody binds strongly and specifically to chicken otoferlin, but does not recognize mammalian otoferlin.

Specificity of the otoferlin IP was assessed by comparison to a control pull-down using a monoclonal antibody to chicken tip-link antigen protocadherin 15 (Goodyear and Richardson, 2003; Kazmierczak et al., 2007). Eluates of both otoferlin and the control IPs were analyzed by LC-MS/MS. Otoferlin was specifically and efficiently immunoprecipitated with the HSC1 antibody and 161 otoferlin peptides were identified covering $74 \%$ of the chicken utricle protein sequence (Supplementary Figure S1). No otoferlin peptides were found in the control pull-down.

In 11 out of 11 independent IPs, CaMKII $\delta$ co-purified with otoferlin, with at least 4 peptides, $95 \%$ peptide threshold, and $99.9 \%$ protein probability. CaMKII $\delta$ was not co-purified in the control IPs.

CaMKII $\delta$ is an important modulator of synapses, but has not been described to play a role in the inner ear. We therefore aimed to find out whether CaMKII $\delta$ interacts with otoferlin and phosphorylates it in mammalian auditory hair cells.

We investigated the localization and expression of all CaMKIIs in mammalian IHCs using immunohistochemistry (Figure 1) and PCR (Figure 2).

In mouse organs of Corti at P14 we found hardly any immunolabelling for CaMKII $\alpha$, CaMKII $\beta$ or CaMKII $\gamma$ within the cytoplasm of IHCs, which were co-labeled for otoferlin (Figures 1A-C). Immunolabelling against CaMKIIB, CaMKII $\gamma$ and CaMKII $\delta$ appeared outside of IHCs, possibly in efferent and/or afferent synaptic boutons (Figures 1B-D). For CaMKII $\delta$, immunoreactivity could be detected also within the cytoplasm of IHCs (Figure 1D). To narrow down the localization of CaMKII $\delta$ at the synapse, we co-labeled with the ribbon protein Ctbp2 and the postsynaptic marker PSD95. Immunolabelling revealed CaMKII $\delta$ to localize close to PSD95, at the opposite side of the ribbon (Figure 1E), indicating a postsynaptic localization of CaMKII $\delta$ in afferent synaptic boutons, in addition to the cytoplasmic localization within the IHCs.

To test for different CaMKII mRNA transcripts in IHCs, we designed PCR primers specific for each of the four CaMKII genes. Suitability to amplify the respective CaMKII transcripts was confirmed using mouse brain CDNA as template in realtime PCR with SYBR green (Figure 2E). 3-5 IHCs per sample were collected with a patch pipette and mRNA was reverse transcribed. Only samples displaying TaqMan-PCR signals for bassoon and TATA-binding protein as housekeeping genes were considered for analysis. Real-time PCR experiments revealed the presence of CaMKII $\delta$ transcripts in three independent samples (Figures $2 \mathrm{~A}-\mathrm{C}$ ). CaMKII $\gamma$ mRNA could be detected in one out of three samples (Figure 2C), while transcripts from CaMKII $\alpha$ and CaMKII $\beta$ could not be amplified in any IHC sample (Figure 2E). Therefore, we conclude that CaMKII $\delta$ is the predominant CaMKII in rodent IHCs, with a supporting contribution by CaMKII $\gamma$.

\section{A Proximity Ligation Assay Confirms Molecular Interaction of Otoferlin and CaMKIII in Rat Cochlear IHCs}

We next investigated whether CaMKII $\delta$ and otoferlin interact in mammalian IHCs using an immunohistochemistry based in situ proximity ligation assay (PLA) (Figure 3) which detects a $<40 \mathrm{~nm}$ proximity of antibody-labeled proteins (Koos et al., 2014).

First, we validated the PLA with a previously reported interaction of otoferlin with myosin VI (Figure 3; Heidrych et al., 2009; Roux et al., 2009). We applied the PLA in acutely isolated P11 rat organ of Corti explants resulting in discrete fluorescent puncta distributed over the whole IHC body (Figure 3B), indicating close proximity $(<40 \mathrm{~nm})$ of otoferlin and myosin VI, likely due to physical interaction. When the PLA assay was done with only one primary antibody to otoferlin as a control (Figure 3B, middle panel), no puncta were detected. Similarly, no PLA signals were detectable when we performed the PLA with antibodies to otoferlin and parvalbumin (Figure 3D and cartoon), another hair cell marker that - like myosin VI - labels the whole IHC body but is not described as otoferlin interaction partner (Figure 3C).

Next, we used the PLA to verify a molecular interaction of otoferlin with CaMKII $\delta$ in rat IHCs in situ (Figure 4). A close proximity of both proteins was indicated by fluorescent puncta in IHCs, suggesting CaMKII $\delta$ to be an otoferlin interaction partner in mammalian cochlear IHCs (Figure 4B). PLA puncta also appeared with the pan-CaMKII antibody (Figure 4D), which was raised against the kinase domain that is highly conserved between the four CaMKII genes. No PLA signals were detected in control assays, using anti-CaMKII antibodies only (Figures $4 B, D$, middle panels). 


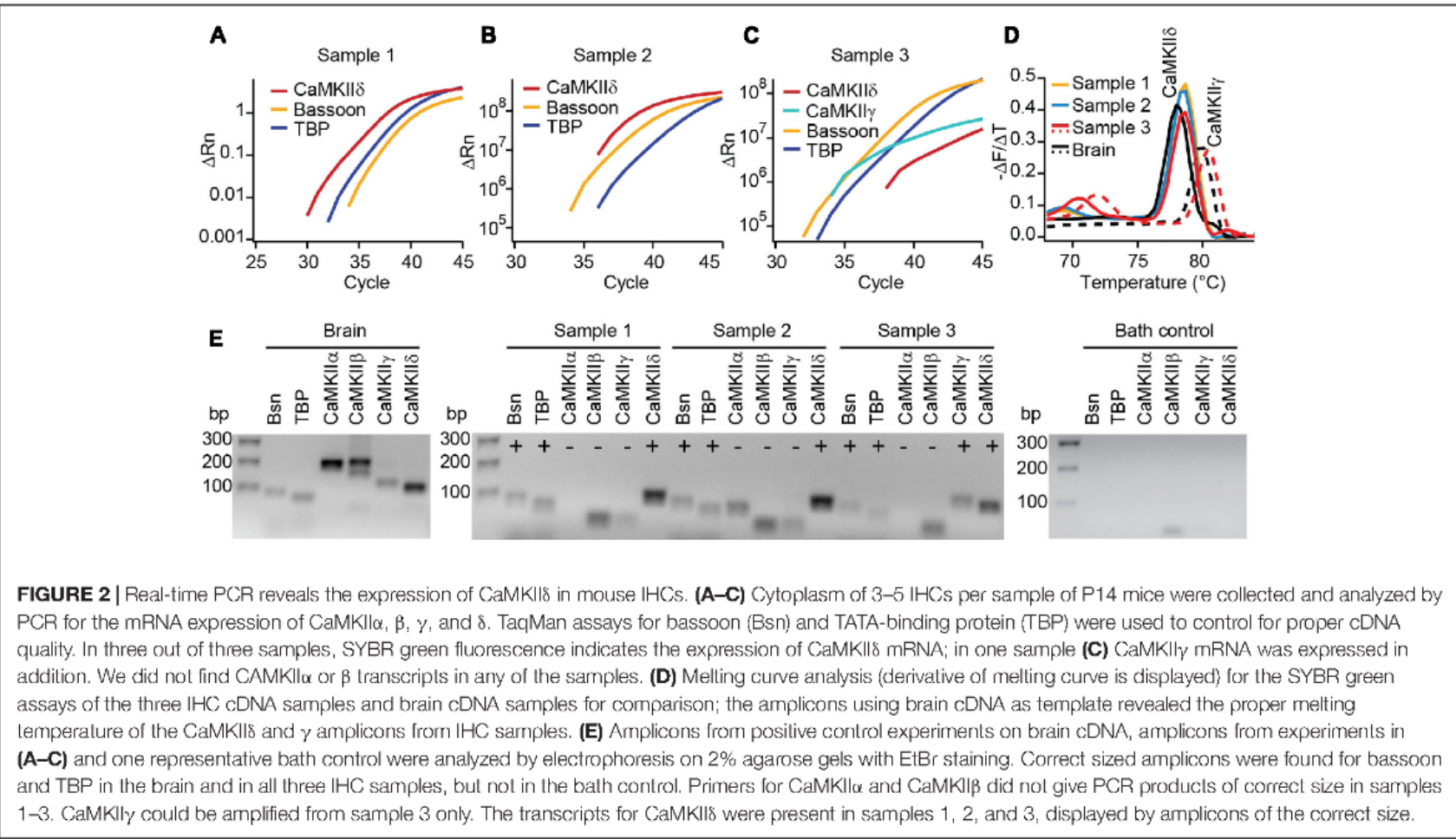

\section{CaMKII $\delta$ Binds Otoferlin In Vitro}

While a positive PLA signal could in principle result from an indirect protein interaction via scaffold proteins, we tested whether CaMKII $\delta$ and otoferlin interact directly in vitro. We expressed two HA-tagged fragments of mouse otoferlin, one comprising the first three $\mathrm{C}_{2}$ domains $\left(\mathrm{C}_{2} \mathrm{ABC}-\mathrm{HA}\right.$, $70 \mathrm{kDa})$, and one comprising the last three $\mathrm{C}_{2}$ domains $\left(\mathrm{C}_{2} \mathrm{DEF}-\mathrm{HA}, 114 \mathrm{kDa}\right)$ as well as full-length mcherry-tagged CaMKII $\delta$ (84 kDa) in HEK293 cells and performed IPs with an anti-HA antibody. In western blots, we identified a $\sim 84 \mathrm{kDa}$ CaMKII $\delta$-mcherry band in the eluate of the co-IPs indicating a co-purification with otoferlin $\mathrm{C}_{2} \mathrm{ABC}$ (Figure 5A). A fainter band was detected in the eluate when CaMKII $\delta$ was co-purified with $\mathrm{C}_{2}$ DEF (Figure 5B), suggesting a weaker interaction. To mimic an interaction with full-length otoferlin, we co-expressed CaMKII $\delta$-mcherry with both $\mathrm{C}_{2} \mathrm{ABC}-\mathrm{HA}$ and $\mathrm{C}_{2} \mathrm{DEF}-\mathrm{HA}$ fragments, resulting again in the co-precipitation of the $\sim 84 \mathrm{kDa}$ band of CaMKII $\delta$-mcherry in the eluate (Figure 5C).

\section{Otoferlin Is Phosphorylated by CaMKIII In Vitro}

We next performed in vitro phosphorylation assays to assess whether CaMKII $\delta$ phosphorylates recombinant otoferlin in a $\mathrm{Ca}^{2+} /$ calmodulin-dependent manner. We used $E$. coli as an expression system because recombinant proteins produced in bacteria lack phosphorylation (Sahdev et al., 2008). We combined purified otoferlin fragments $\left(\mathrm{C}_{2} \mathrm{ABC}\right.$ and $\left.\mathrm{C}_{2} \mathrm{DEF}\right)$ with or without recombinant CaMKII $\delta$ and activated the phosphorylation reaction with $\mathrm{Ca}^{2+}$ and calmodulin. After incubation for $5 \mathrm{~min}$, otoferlin $\mathrm{C}_{2} \mathrm{ABC}$ and $\mathrm{C}_{2} \mathrm{DEF}$ fragments were analyzed for phosphorylation by LC-MS/MS after in-gel trypsinization (Figure 6A). Phosphorylation sites were identified by $80 \mathrm{Da}$ mass shifts in the respective peptides (Supplementary Figure S2).

Only phosphorylation sites identified in three independent experiments were considered. We found that both the $\mathrm{N}$-terminal otoferlin $\mathrm{C}_{2} \mathrm{ABC}$ fragment as well as the C-terminal $\mathrm{C}_{2} \mathrm{DEF}$ fragment were phosphorylated when incubated with CaMKII in vitro and lacked phosphorylation in the absence of the kinase. In total, we identified ten phosphorylation sites (P1 to P10, Figure 6B). Five sites are located within $\mathrm{C}_{2}$ domains of otoferlin, including $\mathrm{T} 448\left(\mathrm{C}_{2} \mathrm{C}\right.$ domain), $\mathrm{S} 985$ ( $\mathrm{C}_{2} \mathrm{D}$ domain), and S1777, S1808, T1860 ( $\mathrm{C}_{2} \mathrm{~F}$ domain; $\mathrm{C}_{2}$ domain borders according to Jiménez and Bashir, 2007). S1184 and T1197 were identified in a region between the $\mathrm{C}_{2} \mathrm{D}$ and the $\mathrm{C}_{2} \mathrm{E}$ domains which has been hypothesized to fold as a $\mathrm{C}_{2}$ domain ( $\mathrm{C}_{2}$ de; Washington and Ward, 2006; Han and Campbell, 2007; Pangršič et al., 2012). Most of the identified phosphorylation sites were found to be conserved between mammalian and non-mammalian otoferlin orthologs (Figure 7), but only a few are conserved within $\mathrm{C}_{2}$ domains of the ferlin protein family (Figure 8). Five out of the ten phosphorylation sites followed the CaMKII consensus sequence R/K-X-X-S/T (White et al., 1998). Noticeably, in six phosphopeptides a hydrophobic leucine was found at the $\mathrm{P}+1$ site of the phosphoserine or threonine (indicated in blue in Figures 7,8 ), displaying a preferred residue for CaMKII recognition (Stokoe et al., 1993). 
A

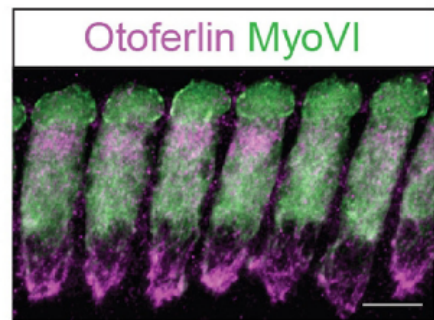

B

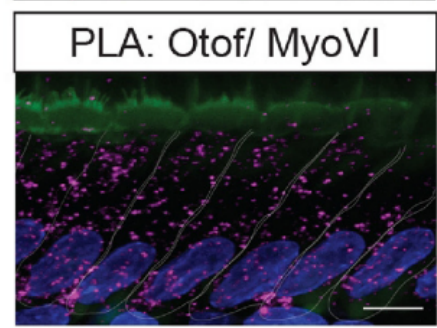

C

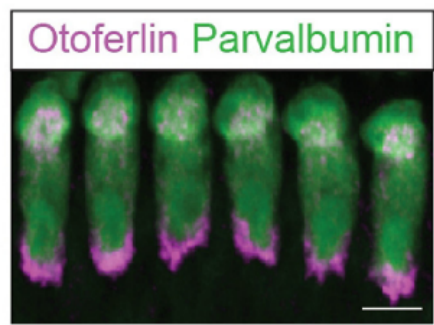

D

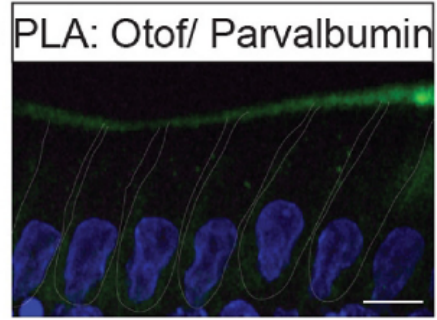

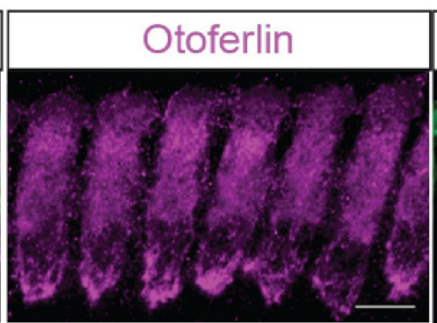
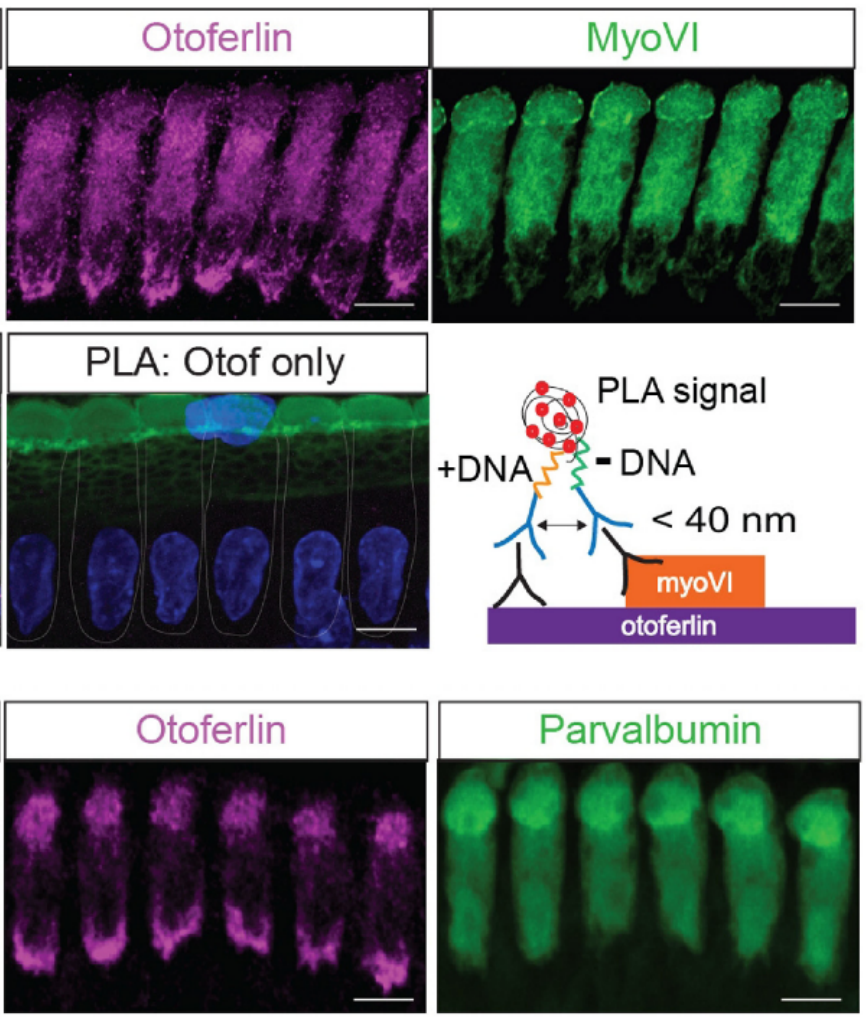

no

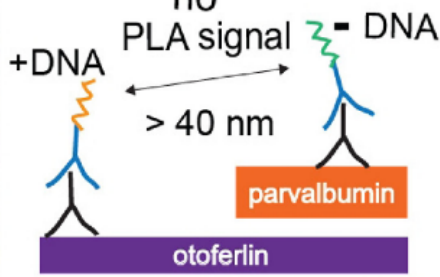

FIGURE 3 | Validation of a proximity ligation assay (PLA) by visualization of the protein interaction of otoferlin and myosin VI in situ. (A) Z-projections of confocal sections of IHCs from a whole-mount explant of a P11 rat organ of Corti, immunolabeled for otoferlin and myosin VI. (B) Primary antibodies from (A) were used for a PLA (see cartoon) to detect in situ protein interactions (magenta punctae in left panel) of otoferlin with myosin VI within $40 \mathrm{~nm}$ distance. No PLA puncta were detected when only one primary antibody to otoferlin was used for PLA (middle panel). (C,D) Immunohistochemistry and negative control for the PLA protocol. (C) Z-projections of confocal sections of IHCs from a P11 rat organ of Corti whole-mount explant, immunolabeled for otoferlin and parvalbumin. (D) Primary antibodies from (C) were used for a PLA. No PLA puncta were detectable suggesting a lack of otoferlin-parvalbumin interaction. In (B,D) cell nuclei were stained with DAPI (blue), hair cell stereocilia with Phalloidin (green). Scale bar, $10 \mu \mathrm{m}$.

\section{Phosphorylation by CaMKII $\delta$ Alters the $\mathrm{Ca}^{2+}$ Affinity of Recombinant Otoferlin $\mathrm{C}_{2} \mathrm{C}$ and $\mathrm{C}_{2} \mathrm{~F}$ Domains}

According to the comparison between ferlin protein family members, the position of the phosphorylated threonine at P1 is occupied by negatively charged aspartate residues in dysferlin and myoferlin (Figure 8), which are positioned in the top loops of the $\mathrm{C}_{2}$ domain, just next to aspartate residues predicted to coordinate $\mathrm{Ca}^{2+}$ (Jiménez and Bashir, 2007). We therefore addressed the influence of CaMKII 8 phosphorylation on the $\mathrm{Ca}^{2+}$ affinity of the recombinant otoferlin $\mathrm{C}_{2} \mathrm{C}$ domain. To mimic the negative charge introduced by phosphorylation, we mutated threonine residue T448 into an aspartate residue (T448D). $\mathrm{Ca}^{2+}$ affinity was assessed by microscale thermophoresis (van den Bogaart et al., 2012) (Figure 9).

For the non-phosphorylated $\mathrm{C}_{2} \mathrm{C}$ domain no changes in fluorescence were detected for $\mathrm{Ca}^{2+}$ concentrations between $6 \mu \mathrm{M}$ and $200 \mathrm{mM}$ compared to the negative control carried out in the presence of $500 \mathrm{mM}$ EDTA (Figure 9A). Thus, either $\mathrm{Ca}^{2+}$ binding occurred but did not change the thermophoresis signal, or the non-phosphorylated $\mathrm{C}_{2} \mathrm{C}$ domain did not bind to $\mathrm{Ca}^{2+}$ in this assay. Accordingly, the recombinant 
A
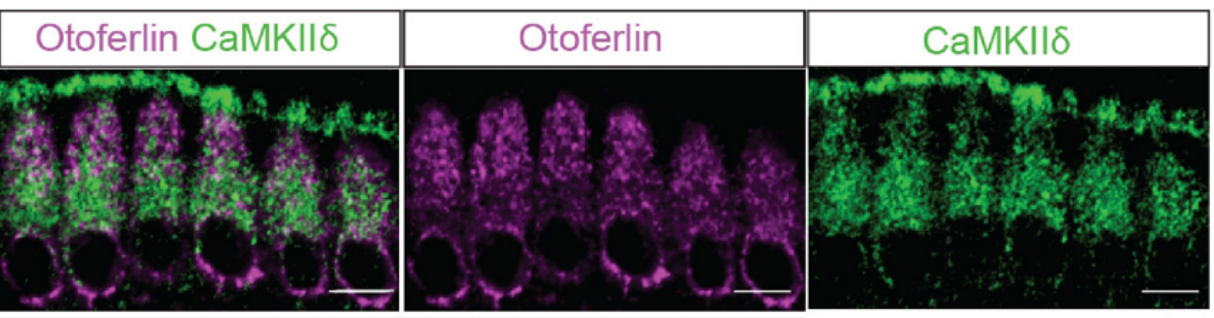

B

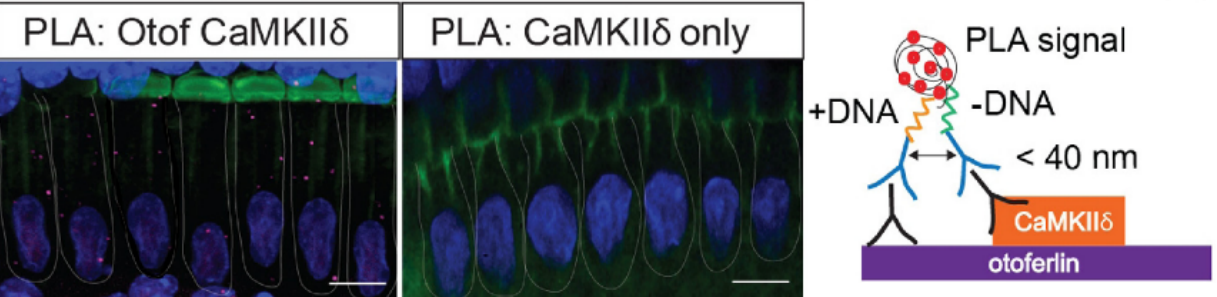

C
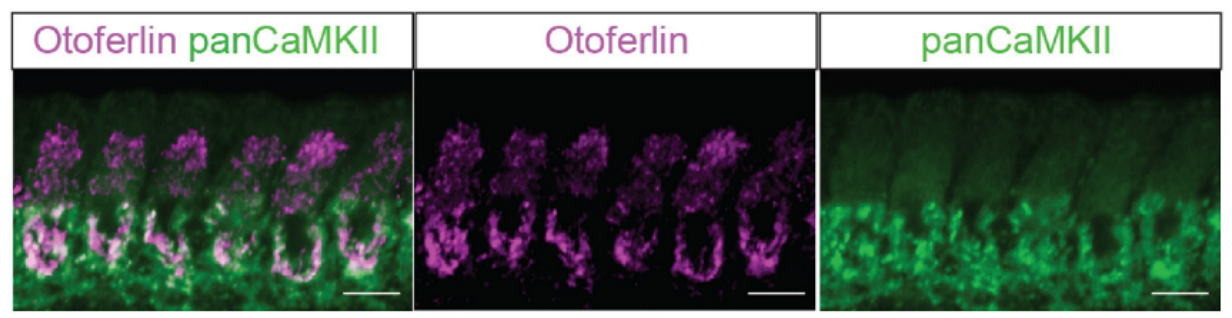

D
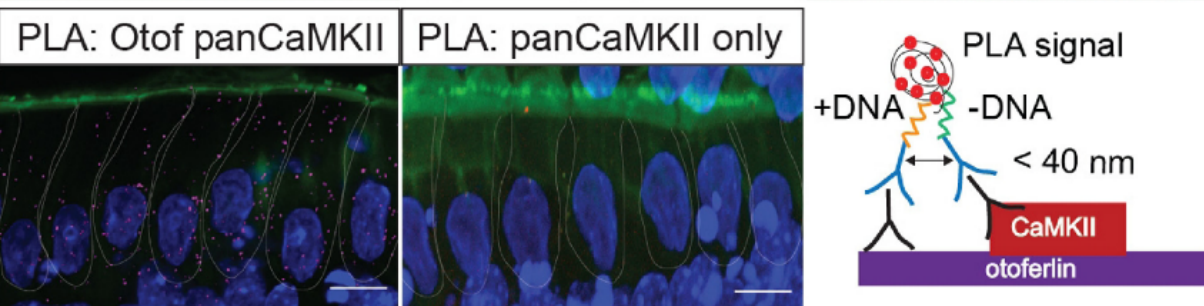

FIGURE 4 | in situ interaction of otoferlin and CaMKII demonstrated by PLA. Z-projections of five confocal sections of IHCs from whole-mount explants of P11 rat organ of Corti, immunolabeled for otoferlin and CaMKII (A) and otoferlin and pan-CaMKII, respectively (C). The same primary antibodies were used for PLA to detect in situ protein interactions of otoferlin with CaMKIIS (B) or all CaMKIl isoforms (D) using the pan-CaMKI. Magenta punctae display locations of close proximity of the two antibodies. No puncta were detected when only one primary antibody to CaMKIII (B, middle panel) or pan-CaMKII (D. middle panel) were used for PLA as control. Blue: DAPI, green in (B,D): Phalloidin. Scale bar, $10 \mu \mathrm{m}$.

otoferlin $\mathrm{C}_{2} \mathrm{ABC}$ construct revealed no change in thermophoresis signal, suggesting lack of $\mathrm{Ca}^{2+}$ binding (Figure 9A). For the phosphomimetic $\mathrm{C}_{2} \mathrm{C}$ domain (T448D) we observed a fluorescence change above the one triggered by $\mathrm{Ca}^{2+}$ plus EDTA (Figure 9B). Curve fitting resulted in an apparent dissociation constant $\left(\mathrm{K}_{\mathrm{Ca}}\right)$ of $8.7 \pm 2.8 \mathrm{mM}$. Hence, phosphorylation by CaMKII $\delta$ most likely converts the $\mathrm{C}_{2} \mathrm{C}$ domain from a non- $\mathrm{Ca}^{2+}$ binding into a $\mathrm{Ca}^{2+}$ binding $\mathrm{C}_{2}$ domain (Figure 9C), although with rather low $\mathrm{Ca}^{2+}$ affinity.

Next, we assessed the effect of phosphorylation on $\mathrm{Ca}^{2+}$ affinity of the $\mathrm{C}_{2} \mathrm{~F}$ domain. For the non-phosphorylated $\mathrm{C}_{2} \mathrm{~F}$ domain, we found an apparent dissociation constant for $\mathrm{Ca}^{2+}$ of $402 \pm 54 \mu \mathrm{M}$, which was abolished in the presence of
EDTA (Figure 9D). Using $\mathrm{Mg}^{2+}$ instead of $\mathrm{Ca}^{2+}$, we also detected a change in fluorescence, yet a $\mathrm{Mg}^{2+}$ concentration of $20 \mathrm{mM}$ was not sufficient to reach a plateau, indicating a rather low $\mathrm{Mg}^{2+}$ affinity of the $\mathrm{C}_{2} \mathrm{~F}$ domain (Figure 9E). We then mimicked phosphorylation of P8, P9 and P10 by replacing the respective serine/threonine residues by aspartates $(\mathrm{S} 1777 \mathrm{D}$, $\mathrm{S} 1808 \mathrm{D}, \mathrm{T} 1860 \mathrm{D})$. The phosphomimetic $\mathrm{C}_{2} \mathrm{~F}$ domain binds $\mathrm{Ca}^{2+}$ with a $\mathrm{K}_{\mathrm{Ca}}$ of $6.7 \pm 0.7 \mathrm{mM}$ (Figure $9 \mathrm{~F}$, corresponding negative control: Figure 9G). For $\mathrm{Mg}^{2+}$ we obtained an apparent dissociation constant of $16.2 \pm 7.2 \mathrm{mM}$ (Figure 9H), which is at least one order of magnitude above the intracellular concentration of free $\mathrm{Mg}^{2+}(<1 \mathrm{mM})$ (Romani and Scarpa, 1992). To assess the effect of salt concentration on the $\mathrm{C}_{2} \mathrm{~F}$ 


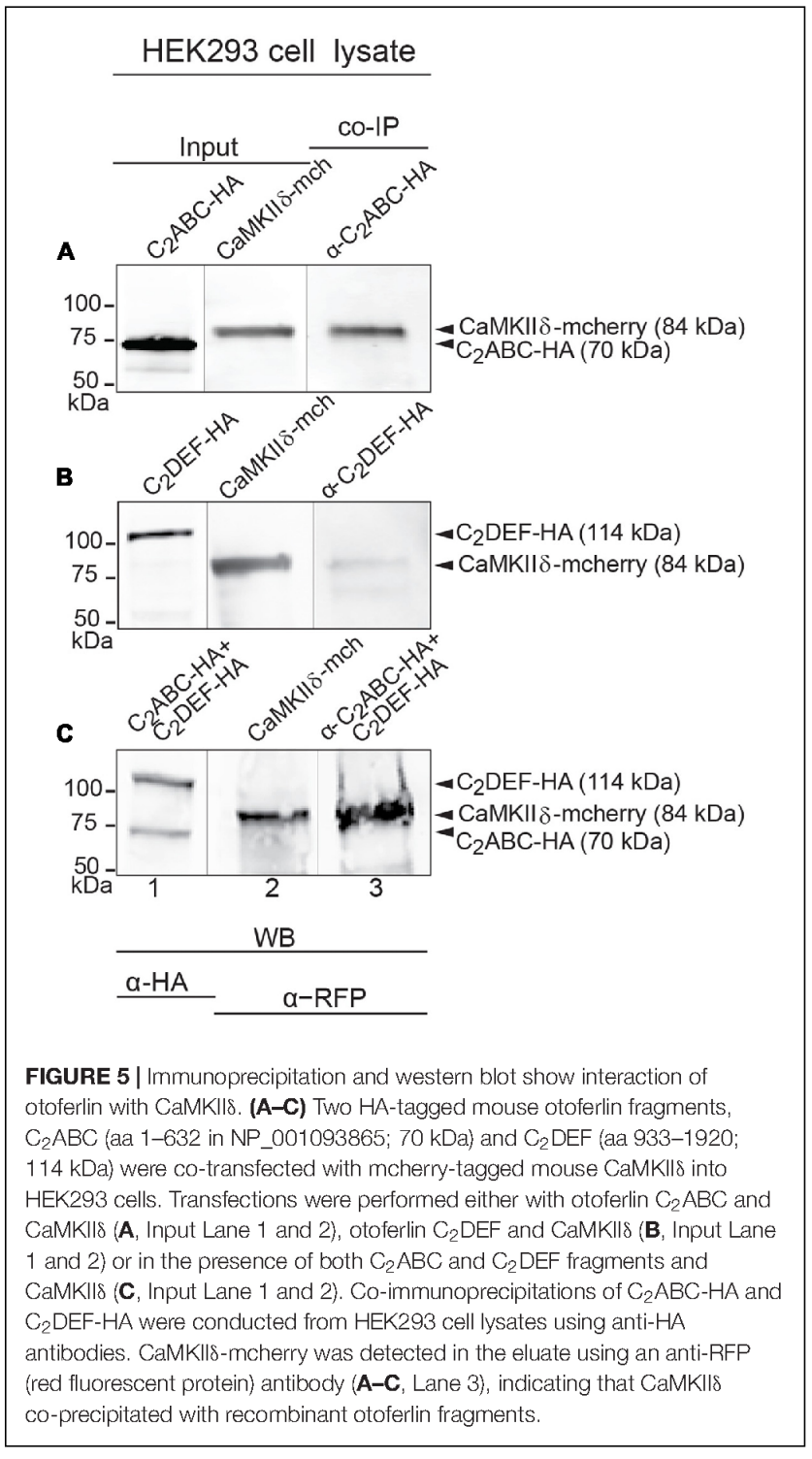

domain stability and hence $\mathrm{Ca}^{2+}$ affinity, we measured the $\mathrm{K}_{\mathrm{Ca}}$ with $150 \mathrm{mM} \mathrm{NaCl}$ as above (Figure 9D) compared to $300 \mathrm{mM}$ $\mathrm{NaCl}$. As indicated in Figure 9I, the fluorescence changes reached a plateau earlier for high salt concentrations, resulting in a lower apparent $\mathrm{K}_{\mathrm{Ca} \text { (high salt) }}$ of $330 \pm 28 \mu \mathrm{M}$.

In summary, phosphomimetic mutations decreased the $\mathrm{Ca}^{2+}$ affinity of the $\mathrm{C}_{2} \mathrm{~F}$ domain by at least one order of magnitude. This suggests that phosphorylation by CaMKII $\delta$ likely results in a lower $\mathrm{Ca}^{2+}$ affinity of the otoferlin $\mathrm{C}_{2} \mathrm{~F}$ domain.

\section{A PLA Reveals Activity-Dependent Phosphorylation of Otoferlin Protein Complexes by CaMKII in IHCs}

Using a PLA to find phosphoserine residues in $<40 \mathrm{~nm}$ distance from otoferlin, we next tested whether otoferlin and/or proteins interacting with otoferlin are phosphorylated in IHCs (Figure 10). To assess whether otoferlin phosphorylation depends on hair cell activity in vivo, we applied low or high external $\mathrm{K}^{+}$solutions to acutely isolated OCs. High $\mathrm{K}^{+}$ depolarizes the plasma membrane leading to opening of voltage gated $\mathrm{Ca}^{2+}$ channels, and the $\mathrm{Ca}^{2+}$-influx triggers $\mathrm{Ca}^{2+}$-induced exocytosis but is also predicted to activate CaMKII by the $\mathrm{Ca}^{2+} /$ calmodulin complex. We found PLA punctae in IHCs at resting conditions (Figure 10B), indicating a basal level of phosphorylated otoferlin or otoferlin interaction partners. After exposure to high $\mathrm{K}^{+}$, we observed more and brighter PLA signals (Figure 10B, middle panel). Comparing mean pixel intensities of the fluorescent PLA signals in resting IHCs with the ones in IHCs after stimulation, we found a four-fold fluorescence increase in high $\mathrm{K}^{+}$-stimulated IHCs (Figure 10C). This suggests a higher degree of otoferlin phosphorylation per otoferlin molecule, a higher number of phosphorylated otoferlin molecules, a higher degree of phosphorylation of proteins interacting with otoferlin, an increased interaction of otoferlin with a phosphorylated protein, or a combination of these possibilities.

To assess whether this increase in phosphorylation of otoferlin or the otoferlin interactome is mediated by CaMKII in rat IHCs, we pre-incubated acutely dissected OCs with the cell-permeable CaMKII inhibitor KN-93, which competitively interacts with the $\mathrm{Ca}^{2+} /$ calmodulin-binding site on CaMKII, (Sumi et al., 1991). In the presence of $\mathrm{KN}-93$, the stimulation-dependent increase in PLA signal was blocked to a large extent (Figures 10B,C), suggesting that activity-dependent phosphorylation of otoferlin or the otoferlin interactome in rat IHCs is indeed mostly due to the CaMKII kinase reaction.

Because of its presumed function in synaptic transmission, we next analyzed if phosphorylated otoferlin or the phosphorylated protein complex localizes to synaptic ribbons. We performed the PLA assay as before with $15 \mathrm{~min}$ high $\mathrm{K}^{+}$-stimulation and co-stained against the ribbon marker Ctbp2 (Figure 10D). PLA puncta, likely reflecting phosphorylated otoferlin and/or otoferlin bound to a phosphorylated protein, did not co-localize with the synaptic ribbons, but were rather found to be in the vicinity or adjacent to each other. This suggests that CaMKII $\delta$ regulates otoferlin activity near the active zone membrane or in endocytotic compartments which are both in close proximity to the synaptic ribbon (Duncker et al., 2013; Neef et al., 2014; Revelo et al., 2014; Jung et al., 2015).

In summary, protein complexes containing otoferlin seem to be phosphorylated adjacent to synaptic ribbons in vivo. Phosphorylation is strongly promoted by hair cell stimulation and can be blocked by a CaMKII inhibitor.

\section{DISCUSSION}

Otoferlin has been implicated in IHC synaptic vesicle fusion, fast synaptic vesicle replenishment potentially including priming and active zone clearance, in vesicle reformation and clathrinmediated endocytosis (Roux et al., 2006; Pangršič et al., 2010; Duncker et al., 2013; Jung et al., 2015; Strenzke et al., 2016), suggesting a multifunctional role in synaptic transmission at the 


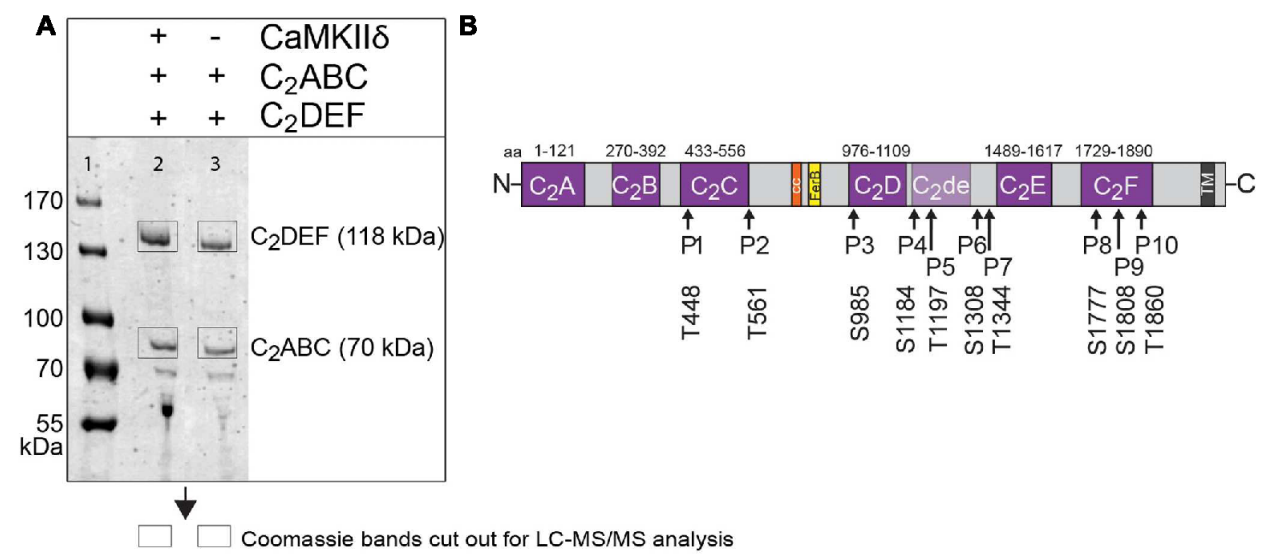

FIGURE 6 | Otoferlin is phosphorylated by CaMKIII in vitro. (A) Otoferlin fragments $\mathrm{C}_{2} \mathrm{ABC}$ (aa. 1-616 in NP_001093865, $70 \mathrm{kDa}$ ) and C2 DEF (aa 908-1932, $118 \mathrm{kDa}$, were expressed in $E$. coli and subjected to an in vitro phosphorylation assay with CaMK $\mid l \delta$ and $\mathrm{Ca}^{2+} / \mathrm{calmodulin}$. Reactions were stopped after 5 min of incubation and proteins were run on a Coomassie gel. Note the slight shift in mass of the fragments between experiment (lane 2) and control without kinase (lane 3). Coomassie stained bands corresponding to otoferlin $\mathrm{C}_{2} \mathrm{DEF}$ and $\mathrm{C}_{2} \mathrm{ABC}$ were cut off the gel and processed for mass spectrometric analysis of otoferlin phosphorylation (Supplementary Figure S2). (B) Three independent experiments as in (A) revealed 10 serine/threonines in otoferlin that were reproducibly phosphorylated by CaMKIll. The putative otoferlin domain topology (in mouse isoform 1; NP_001093865) predicts six $\mathrm{C}_{2}$ domains ( $\mathrm{C}_{2} \mathrm{~A}$ to $\mathrm{C}_{2} \mathrm{~F}_{\text {; }}$ purple), a coiled-coiled domain (orange), a FerB domain (yellow), and a transmembrane domain (TM) (dark gray). Five of the phosphorylation sites are located in $\mathrm{C}_{2}$ domains.

hair cell afferent fiber synapse. Here, we provide evidence that CaMKII $\delta$ phosphorylates otoferlin via direct protein interaction. As phosphorylation altered the $\mathrm{Ca}^{2+}$ affinity of recombinant otoferlin $\mathrm{C}_{2}$ domains, we conclude that CaMKII $\delta$ likely regulates its function.

In a co-purification assay from chicken utricles, we identified CaMKIIS, a $\mathrm{Ca}^{2+} /$ calmodulin-dependent serine/threonine kinase, as a binding partner of otoferlin. A direct interaction of mammalian otoferlin and CaMKII $\delta$ was supported by pull-downs of recombinant otoferlin and CaMKII $\delta$ from HEK293 cells and an immunohistochemistry based PLA on acute explants of OCs demonstrating a close proximity of both proteins $(<40 \mathrm{~nm})$ in IHCs. CaMKII accounts for $1-2 \%$ of all proteins in the brain and is a key modulator of synaptic transmission, mainly through its postsynaptic action (Lisman et al., 2002; Liu and Murray, 2012; Herring and Nicoll, 2016). At the presynaptic site, CaMKII phosphorylates a variety of proteins, including the synaptic vesicle proteins synapsin I, syntaxin $1 \mathrm{~A}$, synaptotagmin $\mathrm{I}$ as well as $\mathrm{Ca}_{v} 1 \mathrm{~L}$-type calcium channels, thereby modulating synaptic vesicle trafficking and exocytosis (Llinás et al., 1991; Fukunaga et al., 1995; Hilfiker et al., 1999; Ohyama et al., 2002; Abiria and Colbran, 2010; Jenkins et al., 2010). However, a recent proteomics study on synaptosomes uncovered no phosphorylation site to be induced by depolarization and $\mathrm{Ca}^{2+}$ entry within a $\mathrm{C}_{2}$ domain (KohansalNodehi et al., 2016). Concordantly, reports about biochemical regulations of $\mathrm{C}_{2}$ domains are rare, e.g., the non- $\mathrm{Ca}^{2+}$ binding $\mathrm{C}_{2}$ domain of a novel $\mathrm{PKC}$ from Aplysia was reported to display higher phospholipid affinity upon phosphorylation (Pepio and Sossin, 2001). The regulation of $\mathrm{Ca}^{2+}$ affinity via $\mathrm{C}_{2}$-domain phosphorylation might then be a unique mechanism in the hair cell synapse and/or for the ferlin protein family, where phosphorylation has not been studied to date. Here, we addressed the biochemical effects of phosphorylation on the $\mathrm{C}_{2} \mathrm{C}$ and the $\mathrm{C}_{2} \mathrm{~F}$ domains of otoferlin only, but in the future it would be interesting to assess the impact of all ten phosphorylation sites on hearing. Interestingly, CaMKII was shown to be involved in sensory adaptation in different sensory modalities. Activated CaMKII is required for adaptation of touch perception in dorsal root ganglion cells which otherwise turns into pain perception (Yu et al., 2015). In the drosophila olfactory system, phosphorylation of synapsin by CaMKII mediates short-term habituation to odors (Sadanandappa et al., 2013). Notably, CaMKII was found to associate with avian utricular and basilar papilla hair cells as well as with synaptic ribbons in bovine retinal photoreceptors (Uthaiah and Hudspeth, 2010; Kantardzhieva et al., 2011) where it phosphorylates syntaxin 3B (Liu et al., 2014), possibly modulating synaptic transmission at sensory ribbon synapses in vestibular and visual sensation. Since long-term adaptation to constant stimuli is assumed not to play a major role in the auditory system, at least for low or medium sound pressure levels, phosphorylation of otoferlin by CaMKII $\delta$ might lead to upregulation of endocytosis and/or vesicle replenishment, to ensure constant signal transmission. On the other hand, CaMKII $\delta$-dependent regulation could possibly result in a sensory desensitization in response to very loud sounds by downregulation of exocytosis, potentially a protection mechanism against noise-induced glutamate toxicity. Notably, individual synapses in one cell respond differently in terms of voltage-dependent $\mathrm{Ca}_{v}$ channel activation and strength of the $\mathrm{Ca}^{2+}$ conductance (Frank et al., 2009; Ohn et al., 2016). It is tempting to speculate that the $\mathrm{Ca}^{2+}$-induced phosphorylation regulates exocytosis, vesicle replenishment and retrieval and/or endocytosis differentially at the synapses for high spontaneous or low spontaneous activity neurons. In addition, $\mathrm{Ca}^{2+}$-induced $\mathrm{Ca}^{2+}$ release (CICR) from intracellular 


\begin{tabular}{|c|c|c|c|c|}
\hline $\begin{array}{l}\text { otoferlin (homo sapiens) } \\
\text { otoferlin (rattus norwegicus) } \\
\text { otoferlin (mus musculus) } \\
\text { otoferlin (danio rerio) } \\
\text { otoferlin (gallus gallus) }\end{array}$ & P1 & 439 & $\begin{array}{l}\text { YRAEGLPRMNTSLMANVKKAF } \\
\text { YRAEGLPRMNTSLMANVKKAF } \\
\text { YRAEGLPRMNTSLMANVKKAF } \\
\text { YRAEGLPRMNTSI MANVKKAF } \\
\text { YRAEGLPRMNTSI MANVKKAL }\end{array}$ & 459 \\
\hline $\begin{array}{l}\text { otoferlin (homo sapiens) } \\
\text { otoferlin (rattus norwegicus) } \\
\text { otoferlin (mus musculus) } \\
\text { otoferlin (danio rerio) } \\
\text { otoferlin (gallus gallus) } \\
\end{array}$ & P2 & 552 & $\begin{array}{l}\text { VNMYGSTRNYTLL DEHQDLNE } \\
\text { VNMYGSTRNYTLL DEHQDLNE } \\
\text { VNMYGSTRNYTLL DEHQDLNE } \\
\text { VNMYGSTRNYTLMDEHQDLNE } \\
\text { VNMYGSTRNYTLMDEHQELNE }\end{array}$ & 572 \\
\hline $\begin{array}{l}\text { otoferlin (homo sapiens) } \\
\text { otoferlin (rattus norwegicus) } \\
\text { otoferlin (mus musculus) } \\
\text { otoferlin (danio rerio) } \\
\text { otoferlin (gallus gallus) }\end{array}$ & P3 & 979 & $\begin{array}{l}\text { QLRAHMYQARSLFAADSSGLS } \\
\text { QLRAHMYQARSLFAADSTGLS } \\
\text { QLRAHMYQARSLFAADSSGLS } \\
\text { QLRAHMYQARSLFAADTSGLS } \\
\text { QLRAHMYQARSLFAADSSGLS }\end{array}$ & 999 \\
\hline $\begin{array}{l}\text { otoferlin (homo sapiens) } \\
\text { otoferlin (rattus norwegicus) } \\
\text { otoferlin (mus musculus) } \\
\text { otoferlin (danio rerio) } \\
\text { otoferlin (gallus gallus) } \\
\end{array}$ & P4/P5 & 1188 & $\begin{array}{l}\text { IECAGKGVQSSLIHNYKKNPNFNT } \\
\text { IECAGKGVQSSLIHNYKKNPNFNT } \\
\text { IECAGKGVQSSLIHNYKKNPNFNT } \\
\text { IECAGKGVQSALIQNYKKNPNFST } \\
\text { IECAGKGVQSALIQNYKKNPNFST }\end{array}$ & $\begin{array}{l}\text { TLVKWFEV } \\
\text { TLVKWFEV } \\
\text { TLV } \\
\text { TLVKWFEV } \\
\text { TLVKWFEV } \\
\text { TLVKWFEV } \\
\end{array}$ \\
\hline $\begin{array}{l}\text { otoferlin (homo sapiens) } \\
\text { otoferlin (rattus norwegicus) } \\
\text { otoferlin (mus musculus) } \\
\text { otoferlin (danio rerio) } \\
\text { otoferlin (gallus gallus) }\end{array}$ & P6 & 1323 & 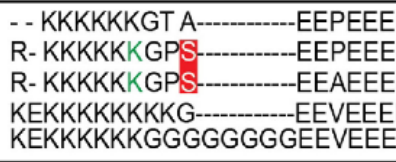 & $\begin{array}{l}\text { EPDE } 1343 \\
\text { EPPDE } \\
\text { EPPDE } \\
\text { EPPDE } \\
\text { EPPDE }\end{array}$ \\
\hline $\begin{array}{l}\text { otoferlin (homo sapiens) } \\
\text { otoferlin (rattus norwegicus) } \\
\text { otoferlin (mus musculus) } \\
\text { otoferlin (danio rerio) } \\
\text { otoferlin (gallus gallus) } \\
\end{array}$ & P7 & 1359 & $\begin{array}{l}\text { MKEQLRQQEPSGIDLEEKEEV } \\
\text { MKEQLRQHETSGIDLEEKEEM } \\
\text { MKEQLRQHETSGTDLEEKEEM } \\
\text { MMENLRAQEAAQAEAEEREDL } \\
\text { MKEQLRQQEAAAAEAEEKEDL }\end{array}$ & 1379 \\
\hline $\begin{array}{l}\text { otoferlin (homo sapiens) } \\
\text { otoferlin (rattus norwegicus) } \\
\text { otoferlin (mus musculus) } \\
\text { otoferlin (danio rerio) } \\
\text { otoferlin (gallus gallus) } \\
\end{array}$ & P8 & 1797 & $\begin{array}{l}\text { DKQDTDVHYHSLTGEGNFNW } \\
\text { DKQDTDVHYHSLTGEGNFNW } \\
\text { DKQDTDVHYHSLTGEGNFNW } \\
\text { DKQDTDVHYHSLTGEGNFNW } \\
\text { DKQDTDVHYHSLTGEGNFNW }\end{array}$ & 1816 \\
\hline $\begin{array}{l}\text { otoferlin (homo sapiens) } \\
\text { otoferlin (rattus norwegicus) } \\
\text { otoferlin (mus musculus) } \\
\text { otoferlin (danio rerio) } \\
\text { otoferlin (gallus gallus) } \\
\end{array}$ & P9 & 1828 & $\begin{array}{l}\text { EEKIV ISKKESMFSWDETEYK } \\
\text { EEKIVMSKKESMFSWDETEYK } \\
\text { EEKIVMSKKESMFSWDETEYK } \\
\text { EEKIV ISKKESMFSWDETEYK } \\
\text { EEKIV ISKKESMFSWDETEYK }\end{array}$ & 1848 \\
\hline $\begin{array}{l}\text { otoferlin (homo sapiens) } \\
\text { otoferlin (rattus norwegicus) } \\
\text { otoferlin (mus musculus) } \\
\text { otoferlin (danio rerio) } \\
\text { otoferlin (gallus gallus) }\end{array}$ & P10 & 1880 & $\begin{array}{l}\text { PRGAKTAKQCTMEMATGEVDV } \\
\text { PRGAKTAKQCTMEMATGEVDV } \\
\text { PRGAKTAKQCTMEMATGEVDV } \\
\text { PRGAKTAKQCSL DMVLKEHEL } \\
\text { PRGAKTSKQOSL EMVTNEAEL }\end{array}$ & 1900 \\
\hline
\end{tabular}

FIGURE 7 | Sequence alignment of phosphorylated sites for otoferlin from different species. Sequence alignment of phosphorylated mouse otoferlin peptides (NP_001093865) with human (NP_919224), rat (XP_006239895), zebrafish (NP_001025283), and chicken (XP_420015) otoferlin. As indicated in red, seven of the phosphorylation sites are conserved amongst the species $(P 1, P 2, P 3, P 5, P 8, P 9$, and $P 10)$. P6 and $P 7$ are only found in rat and mouse otoferlin and $P 4$ is conserved amongst human, rat and mouse. Polential CaMKII consensus motifs $(R / K-X-X-S / T)$ are indicaled by green arginines $(R)$ or lysines $(K)$ at the -3 position of the phosphorylation sites. Hydrophobic leucine residues at the +1 position of a phosphoserine or -threonine that were shown to be favored by CaMKIl (White et al. 1998) are labeled in blue.

$\mathrm{Ca}^{2+}$ stores was reported to reduce sustained vesicle release via a so far unknown mechanism (Castellano-Muñoz et al., 2016). The potential involvement of CaMKII $\delta$ in this pathway could regulate otoferlin activity more distally to the active zone.

Five of the ten phosphorylation sites identified by the in vitro CaMKII $\delta$ phosphorylation assay were located within $\mathrm{C}_{2}$ domains of otoferlin. In human myoferlin or dysferlin, the position of the phosphorylated threonine in the $\mathrm{C}_{2} \mathrm{C}$ domain is held by an aspartate residue (Figure 8), which is predicted to form a $\mathrm{Ca}^{2+}$ coordination site (Shao et al., 1998; Ubach et al., 1998; Jiménez and Bashir, 2007). For the non-phosphorylated $\mathrm{C}_{2} \mathrm{C}$ domain and a longer fragment containing $\mathrm{C}_{2} \mathrm{ABC}$ domains, we found no $\mathrm{Ca}^{2+}$ binding which is in accordance with in silico predictions (Jiménez and Bashir, 2007) but contrasts experimental findings from other groups that applied an autofluorescence assay or isothermal titration calorimetry (Johnson and Chapman, 2010; Padmanarayana et al., 2014).

For the wild type $\mathrm{C}_{2} \mathrm{~F}$ domain, we determined an apparent dissociation constant for $\mathrm{Ca}^{2+}$ of 330 and $402 \mu \mathrm{M}$ for high and low salt buffer, respectively, which is in good agreement with a 


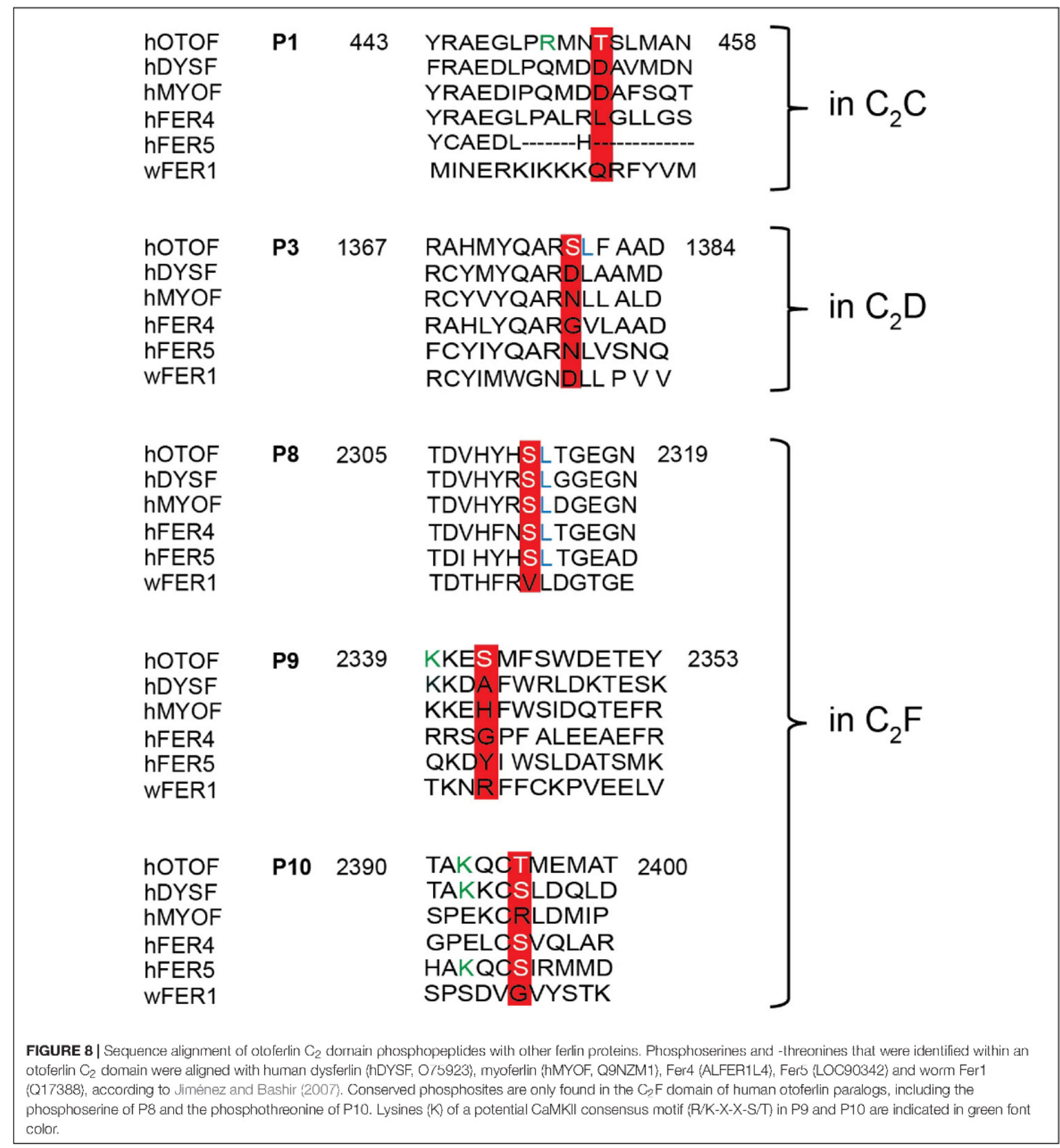

$\mathrm{K}_{D(\mathrm{Ca})}$ of $267 \mu \mathrm{M}$ for the $\mathrm{C}_{2} \mathrm{~F}$ domain determined by isothermal titration calorimetry in high salt buffer (Ramakrishnan et al., 2014). However, other groups reported the $\mathrm{Ca}^{2+}$ affinity of the $\mathrm{C}_{2} \mathrm{~F}$ domain to be $\sim 25 \mu \mathrm{M}$ using isothermal titration calorimetry, and $\sim 20 \mu \mathrm{M}$ assessing autofluorescence changes (Johnson and Chapman, 2010; Padmanarayana et al., 2014). Note that our $\mathrm{C}_{2} \mathrm{~F}$ fragment's size (aa 1695-1934) is different from the ones used in the aforementioned studies (aa 1720-1885), which would lack one $\beta$-strand according to in silico predictions (Jiménez and Bashir, 2007). In the $<1 \mu \mathrm{M}$ to $<100 \mu \mathrm{M}$ range, no MST signal was detected, indicating either the absence of such a high affinity $\mathrm{Ca}^{2+}$ binding site in our $\mathrm{C}_{2} \mathrm{~F}$ fragment or a $\mathrm{Ca}^{2+}$ binding event which did not result in a detectable change (as for Synaptotagmin-1 $\mathrm{C}_{2} \mathrm{~A}$, van den Bogaart et al., 2012). 


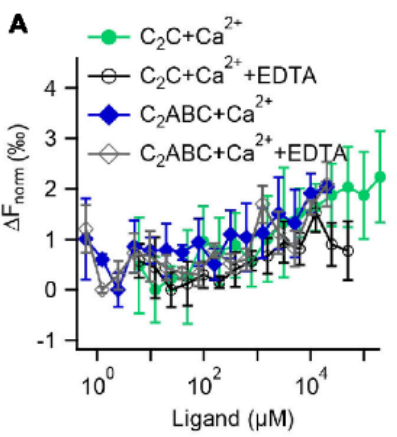

D
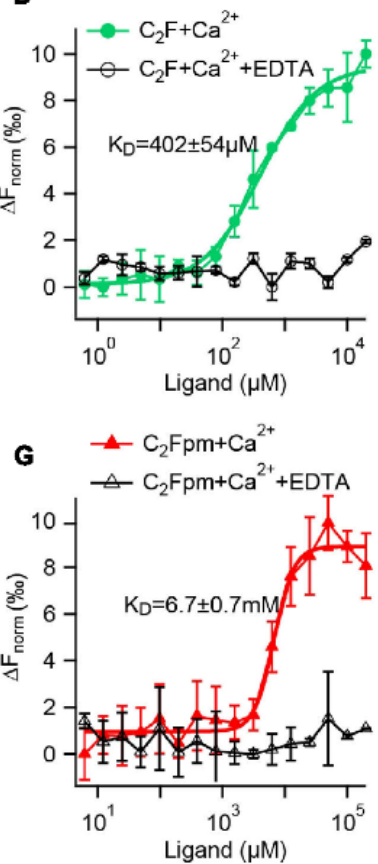

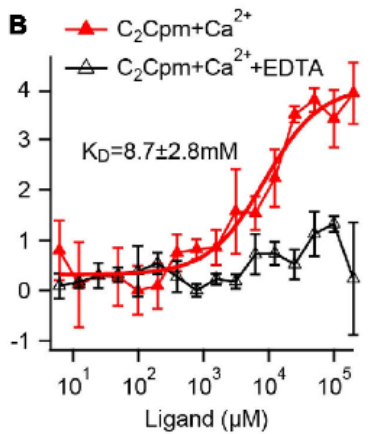

$\mathbf{E}$
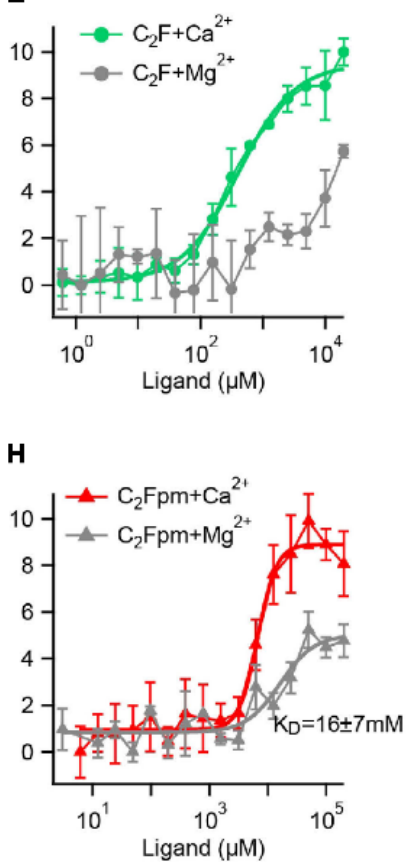

C

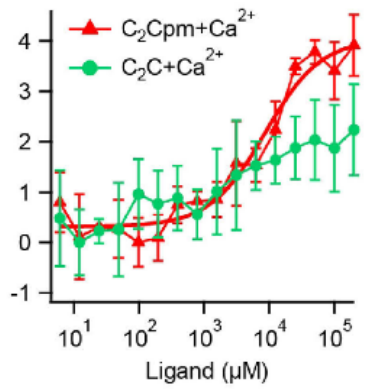

$\mathbf{F}$
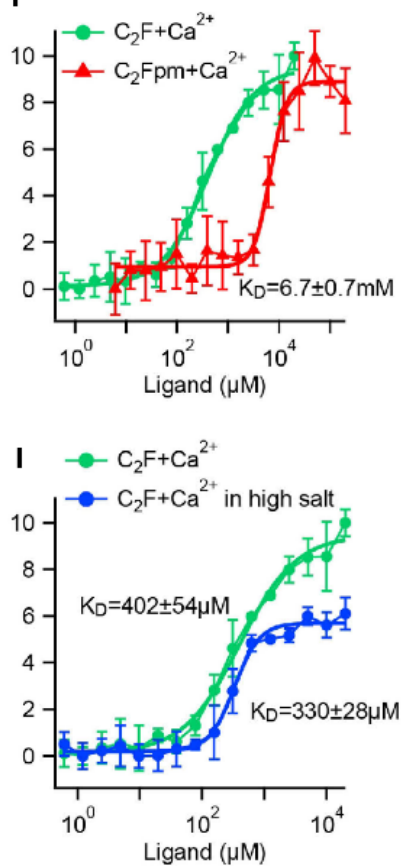

FIGURE 9 | Microscale thermophoresis (MST) assays reveal that phosphorylation increases the $\mathrm{Ca}^{2+}$ affinity of the $\mathrm{C}_{2} \mathrm{C}$ domain and reduces the Ca ${ }^{2+}$ affinity of the $\mathrm{C}_{2} \mathrm{~F}$ domain. (A-I) Fluorescence changes after infrared laser mediated heating of the sample indicate binding of a ligand. Data points are mean values $\pm \mathrm{SD}$ for $n=3$ technical replicates each. $K_{D} S$ were acquired by Hill fitting (solid lines) in IGOR (Wavemetrics). (A) A minor change in fluorescence for the $\mathrm{C}_{2} \mathrm{C}$ domain or a fragment containing the $\mathrm{C}_{2} \mathrm{ABC}$ domains did not differ from the negative controls with EDTA, suggesting no $\mathrm{Ca}^{2+}$ binding. (B) In contrast, the phosphomimetic (pm) $\mathrm{C}_{2} \mathrm{C}$ domain (T449D) showed binding to $\mathrm{Ca}^{2+}$, but with rather low affinity. (C) Direct comparison for the wild-type and the phosphomimetic $\mathrm{C}_{2} \mathrm{C}$ domain illustrates that phosphorylation increases the $\mathrm{Ca}^{2+}$ affinity. (D) The wild-type $\mathrm{C}_{2} \mathrm{~F}$ domain binds to $\mathrm{Ca}^{2+}$; but not when EDTA was present. (E) In comparison, the binding of Mg ${ }^{2+}$ to the $\mathrm{C}_{2} \mathrm{~F}$ domain is much weaker than the binding to $\mathrm{Ca}^{2+}$. (F) Compared to the non-phosphorylated $\mathrm{C}_{2} \mathrm{~F}$ domain, the three phosphomimetic mutations lower $\mathrm{Ca}^{2+}$ affinity by one order of magnitude; $(\mathbf{G})$ no binding occurred in the presence of EDTA. (H) $\mathrm{Mg}^{2+}$ affinity is lower than $\mathrm{Ca}^{2+}$ affinity for the phosphomimetic $\mathrm{C}_{2} \mathrm{~F}$ domain. (I) Measuring in a high salt buffer $(300 \mathrm{mM} \mathrm{NaCl})$ slightly lowers the $\mathrm{K}_{\mathrm{D}}$ for $\mathrm{Ca}^{2+}$.

The $\mathrm{Ca}^{2+}$ concentrations within $\mathrm{Ca}^{2+}$ hotspots at IHC ribbon synapses are estimated to range from $>10 \mu \mathrm{M}$ to $>100 \mu \mathrm{M}$ (Roberts, 1994; Beutner et al., 2001; Wong et al., 2014). Therefore, a $\mathrm{K}_{\mathrm{D}}$ of a few $\mathrm{mM}$ for the $\mathrm{Ca}^{2+}$ binding of the $\mathrm{C}_{2} \mathrm{C}$ phosphomimetic mutant, indicates that even the phosphorylated $\mathrm{C}_{2} \mathrm{C}$ domain likely does not bind $\mathrm{Ca}^{2+}$ in vivo. Also, a dissociation constant of a few hundred $\mu \mathrm{M}$ for the $\mathrm{Ca}^{2+}$ binding of the $\mathrm{C}_{2} \mathrm{~F}$ domain might seem high, yet similar values were observed in vitro for the binding of $\mathrm{Ca}^{2+}$ to the recombinant synaptotagmin-1 $\mathrm{C}_{2} \mathrm{~B}$ domain $\left(\mathrm{K}_{\mathrm{D}} \sim 200 \mu \mathrm{M}\right.$ ) (Fernandez et al., 2001; Radhakrishnan et al., 2009; van den Bogaart et al., 2012).
As the presence of negatively charged phospholipids is known to increase the $\mathrm{Ca}^{2+}$ affinity of the $\mathrm{C}_{2}$ domains of synaptotagmin and protein kinase C (Brose et al., 1992; Guerrero-Valero et al., 2009; van den Bogaart et al., 2012), we speculate that the affinity of the otoferlin $\mathrm{C}_{2}$ domains for $\mathrm{Ca}^{2+}$ also increases in the presence of phospholipid membranes. Furthermore, other posttranslational modifications or protein-protein interactions might affect the $\mathrm{Ca}^{2+}$ affinity. For example, other kinases than those of the CaMKII family might be able to phosphorylate otoferlin. In summary, we hypothesize that $\mathrm{Ca}^{2+}$ likely binds to the nonphosphorylated otoferlin $\mathrm{C}_{2} \mathrm{~F}$ domain in vivo. 
A
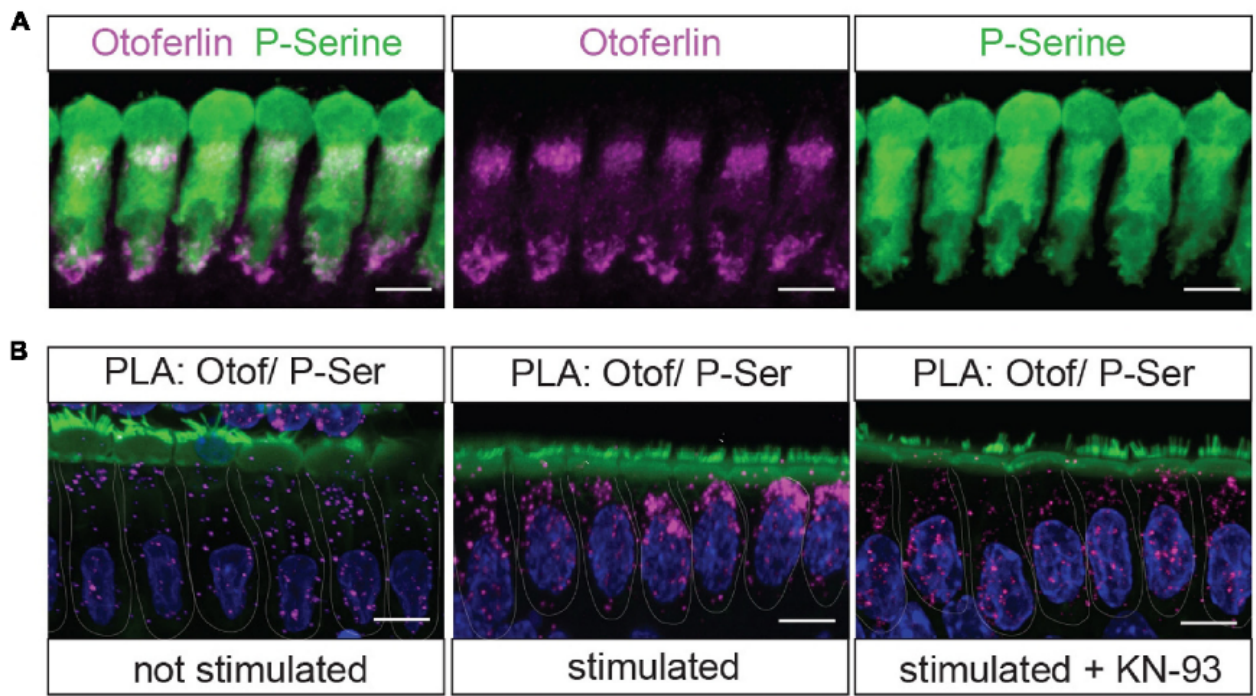

C
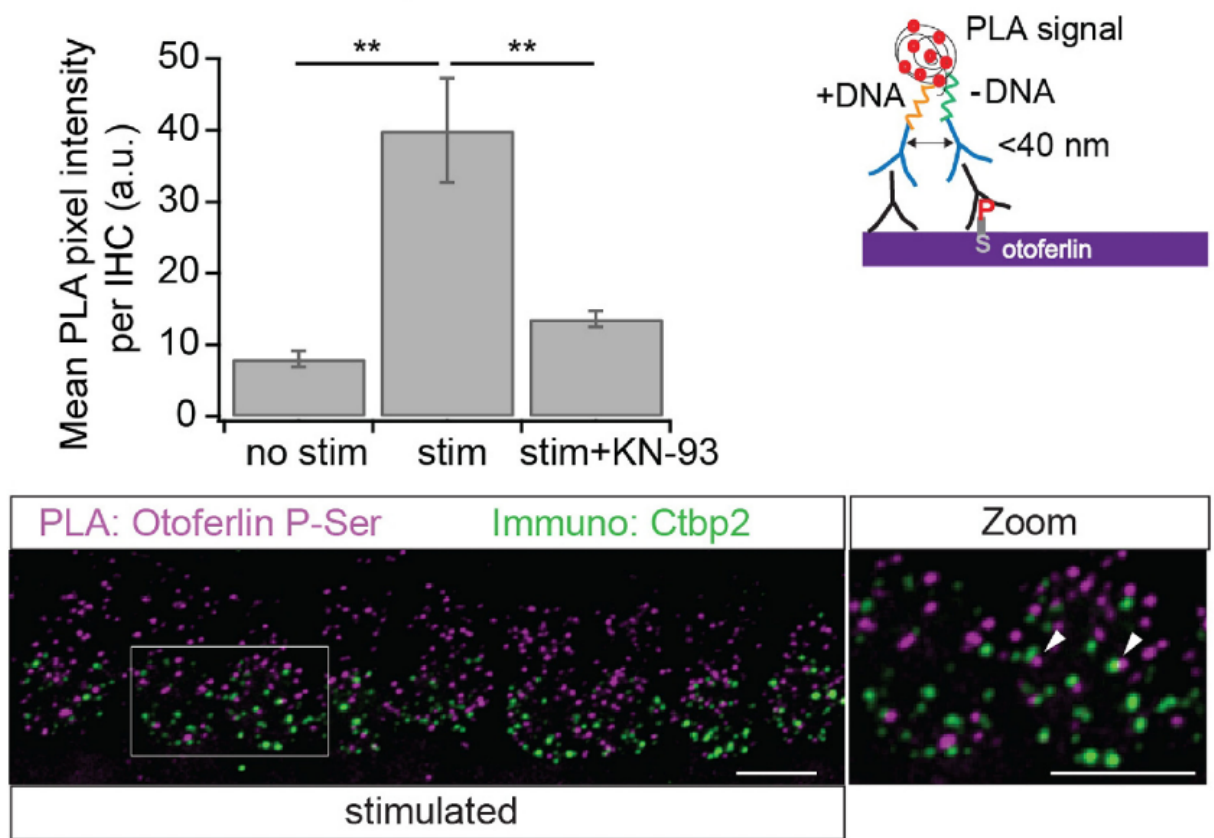

FIGURE 10 | Immunolocalization of phosphoserine residues close to otoferlin in IHCs. (A) Projection of a confocal z-stack of IHCs from a whole-mount explant of a rat P10 OC immunolabeled for otoferlin (magenta) and phosphoserine (green). (B) Primary antibodies from (A) were used to detect phosphoserine residues in $<40 \mathrm{~nm}$ distance from otoferlin in situ by PLA. Nuclei staining in blue (DAPI) and hair cell stereocilia in green (Phalloidin). Magenta PLA punctae indicate phosphoserine residues on otoferlin and/or on a direct interaction partner after 15 min at $37^{\circ} \mathrm{C}$ in low extracellular $\mathrm{KCl}$ solution (left panel). Hair cell stimulation with $40 \mathrm{mM} \mathrm{KCl}$ for $15 \mathrm{~min}$ at $37^{\circ} \mathrm{C}$ (middle panel) appears to increase PLA signals compared to not stimulated hair cells. Incubation with the CaMKII inhibitor KN-93 (right panel) blocks this effect. (C) Quantification of the PLA signal intensities. Compared with resting conditions, hair cell stimulation significantly increases PLA fuorescence intensity (two-tailed $i$-test, $p=0.0017$; three independent experiments, with $30 \mathrm{IHCs}$ analyzed for each condition) and pharmacological inhibition of CaMKII with KN-93 blocks most of this effect (two-tailed $t$-test, $\rho=0.0034 ; N=3$ experiments with 30 IHCs for each condition). Bars show mean $=\mathrm{SD}$; two-tailed t-test; ** $p=0.001-0.01$. (D) Projection of a confocal z-stack of IHCs from a whole-mount explant of rat P9 organs of Corti after PLA assay with primary antibodies to otoferlin and phosphoserine (magenta) and a parallel immunostaining against the ribbon marker Ctbp2 (green) indicates close proximity but no co-localization of phosphorylated otoferlin protein complexes and synaptic ribbons. Scale bars: $10 \mu \mathrm{m}$.

Phosphorylation of the $\mathrm{C}_{2} \mathrm{~F}$ domain, mimicked here by replacing phosphorylated serine/threonine residues by aspartates, resulted in a more than 10 -fold reduction in $\mathrm{Ca}^{2+}$ affinity. Even in the presence of phospholipid membranes, we assume that the phosphomimetic $\mathrm{C}_{2} \mathrm{~F}$ domain is not capable of binding $\mathrm{Ca}^{2+}$ in IHCs. 
Although phosphomimetic mutations might differ from actually phosphorylated serine/threonine residues, our data suggest that phosphorylation by CaMKII $\delta$ renders the $\mathrm{C}_{2} \mathrm{~F}$ domain grossly $\mathrm{Ca}^{2+}$-insensitive in IHCs, providing a molecular mechanism for the suggested regulation of otoferlin activity by CaMKII 8 .

The PLA displaying phosphoserine residues in close proximity $(<40 \mathrm{~nm})$ to otoferlin showed an increase signal upon stimulation of the IHCs (Figure 10). This experimental setting cannot distinguish between phosphorylated serines within otoferlin and those on proteins interacting with otoferlin. However, since we demonstrate that otoferlin and CaMKII $\delta$ interact and a $5 \mathrm{~min}$ co-incubation of both proteins in vitro is sufficient to trigger the phosphorylation of otoferlin in ten residues (five of which are serines), we presume that at least part of the PLA signal indicates the phosphorylation of otoferlin itself. Nevertheless, even considering that the assay is detecting phosphoserines on proteins interacting with otoferlin, this points toward a CaMKII-dependent regulation of the otoferlin interactome, probably resulting in the regulation of the IHC synaptic activity.

\section{CONCLUSION}

Upon hair cell stimulation, $\mathrm{Ca}^{2+}$ entering the IHCs activates CaMKII $\delta$ which phosphorylates otoferlin. We hypothesize that this phosphorylation renders the $\mathrm{C}_{2} \mathrm{~F}$ domain of otoferlin $\mathrm{Ca}^{2+}$ insensitive under physiological conditions, which might regulate the kinetics of exocytosis, vesicle replenishment and/or endocytosis.

\section{REFERENCES}

Abiria, S. A., and Colbran, R. J. (2010). CaMKII associates with CaV1.2 L-type calcium channels via selected beta subunits to enhance regulatory phosphorylation. J. Neurochem. 112, 150-161. doi: 10.1111/j.1471-4159.2009 06436.x

Beutner, D., Voets, T., Neher, E., and Moser, T. (2001). Calcium dependence of exocytosis and endocytosis at the cochlear inner hair cell afferent synapse. Neuron 29, 681-690. doi: 10.1016/S0896-6273(01)00243-4

Brose, N., Petrenko, A. G., Südhof, T. C., and Jahn, R. (1992). Synaptotagmin: a calcium sensor on the synaptic vesicle surface. Science 256, 1021-1025. doi: $10.1126 /$ science. 1589771

Castellano-Muñoz, M., Schnee, M. E., and Ricci, A. J. (2016). Calciuminduced calcium release supports recruitment of synaptic vesicles in auditory hair cells. J. Neurophysiol. 115, 226-239. doi: 10.1152/jn.00 559.2015

Duncker, S. V., Franz, C., Kuhn, S., Schulte, U., Campanelli, D., Brandt, N., et al. (2013). Otoferlin couples to clathrin-mediated endocytosis in mature cochlear inner hair cells. J. Neurosci. 33, 9508-9519. doi: 10.1523/JNEUROSCI.5689-12. 2013

Fernandez, I., Arac, D., Ubach, I., Gerber, S. H., Shin, O., Gao, Y., et al (2001). Three-dimensional structure of the synaptotagmin $1 \mathrm{C} 2 \mathrm{~B}-$ domain synaptotagmin 1 as a phospholipid binding machine. Neuron 32, 1057-1069. doi: 10.1016/S0896-6273(01)00548-7

\section{AUTHOR CONTRIBUTIONS}

MH, ER, SH, RF, and AR designed study. MH, SM, AC, FG, CA, and ER performed experiments and analyzed data. MH, ER, SM, and $\mathrm{AC}$ wrote manuscript and prepared figures. $\mathrm{MH}, \mathrm{ER}, \mathrm{RF}$, $\mathrm{SH}$, and $\mathrm{AR}$ acquired funding.

\section{FUNDING}

This work was supported by a research stipend of the German research foundation (Deutsche Forschungsgemeinschaft, DFG) to $\mathrm{MH}$ and by collaborative research center 889 , project $\mathrm{A} 4$, of the Deutsche Forschungsgemeinschaft to ER and RF. Further financial support was provided by the National Institute of Health, NIH RO1 DC004563 to SH and RO1 DC009913 to AR. Mass spectrometry was supported by Award Number S10RR027425 from the NCRR.

\section{ACKNOWLEDGMENTS}

We thank Dr. Christine Petit, Institute Pasteur, Paris, France for providing us with the mouse cDNA of full-length otoferlin. We thank Heide Marie Resch for help with MST analysis and Tobias Moser for helpful discussion. Parts of the data are subject to the Ph.D. thesis of SM, archived by the University of Göttingen.

\section{SUPPLEMENTARY MATERIAL}

The Supplementary Material for this article can be found online at: http://journal.frontiersin.org/article/10.3389/fnsyn. 2017.00013/full\#supplementary-material

Frank, T., Khimich, D., Neef, A., and Moser, T. (2009). Mechanisms contributing to synaptic $\mathrm{Ca}^{2+}$ signals and their heterogeneity in hair cells. Proc. Natl. Acad. Sci. U.S.A. 106, 4483-4488. doi: 10.1073 /pnas. 0813213106

Fukunaga, K., Muller, D., and Miyamoto, E. (1995). Increased phosphorylation of $\mathrm{Ca}^{2+} /$ calmodulin-dependent protein kinase II and its endogenous substrates in the induction of long-term potentiation. J. Biol. Chem. 270, 6119-6124. doi: $10.1074 / \mathrm{jbc} .270 .11 .6119$

Glowatzki, E., and Fuchs, P. A. (2002). Transmitter release at the hair cell ribbon synapse. Nat. Neurosci. 5, 147-154. doi: 10.1038/nn796

Goodyear, R. J., Gates, R., Lukashkin, A. N., and Richardson, G. P. (1999). Haircell numbers continue to increase in the utricular macula of the early posthatch chick. J. Neurocytol. 28, 851-861. doi: 10.1023/A:1007070121751

Goodyear, R. J., Legan, P. K., Christiansen, J. R., Xia, B., Korchagina, J., Gale, J. E., et al. (2010). Identification of the hair cell soma- 1 antigen, HCS-1, as otoferlin. J. Assoc. Res. Otolaryngol. 11, 573-586. doi: 10.1007/s10162-010-0231-6

Goodyear, R. J., and Richardson, G. P. (2003). A novel antigen sensitive to calcium chelation that is associated with the tip links and kinocilial links of sensory hair bundles. J. Neurosci. 23, 4878-4887.

Guerrero-Valero, M., Ferrer-Orta, C., Querol-Audí, J., Marin-Vicente, C., Fita, I., Gómez-Fernández, J. C., et al. (2009). Structural and mechanistic insights into the association of PKCalpha-C2 domain to PtdIns $(4,5) \mathbf{P}_{2}$. Proc. Natl. Acad. Sci. U.S.A. 106, 6603-6607. doi: 10.1073/pnas.0813099106

Han, R., and Campbell, K. P. (2007). Dysferlin and muscle membrane repair. Curr. Opin. Cell Biol. 19, 409-416. doi: 10.1016/j.ceb.2007.07.001 
Appendix | 163

Heidrych, P., Zimmermann, U., Breß, A., Pusch, C. M., Ruth, P., Pfister, M., et al. (2008). Rab8b GT Pase, a protein transport regulator, is an interacting partner of otoferlin, defective in a human autosomal recessive deafness form. Hum. Mol. Genet. 17, 3814-3821. doi: 10.1093/hmg/ddn279

Heidrych, P., Zimmermann, U., Kuhn, S., Franz, C., Engel, J., Duncker, S. V., et al. (2009). Otoferlin interacts with myosin VI: implications for maintenance of the basolateral synaptic structure of the inner hair cell. Hum. Mol. Genet. 18, 2779-2790. doi: $10.1093 / \mathrm{hmg} / \mathrm{ddp} 213$

Heller, S., Bell, A. M., Denis, C. S., Choe, Y., and Hudspeth, A. J. (2002). Parvalbumin 3 is an abundant $\mathrm{Ca} 2+$ buffer in hair cells. J. Assoc. Res. Otolaryngol. 3, 488-498. doi: $10.1007 / \mathrm{s} 10162-002-2050-\mathrm{x}$

Herget, M., Scheibinger, M., Guo, Z., Jan, T. A., Adams, C. M., Cheng, A. G., et al. (2013). A simple method for purification of vestibular hair cells and non-sensory cells, and application for proteomic analysis. PLOS ONE 8:e66026. doi: 10.1371 /journal.pone.0066026

Herring, B. E., and Nicoll, R. A. (2016). Long-term potentiation: from CaMKII to AMPA receptor trafficking. Annu. Rev. Physiol. 78, 351-365. doi: 10.1146/ annurev-physiol-021014-071753

Hickman, T. T., Liberman, M. C., and Jacob, M. H. (2015). Adenomatous polyposis coli protein deletion in efferent olivocochlear neurons perturbs afferent synaptic maturation and reduces the dynamic range of hearing. J. Neurosci. 35, $9236-$ 9245. doi: 10.1523/JNEUROSCI.4384-14.2015

Hilfiker, S., Pieribone, V. A., Nordstedt, C., Greengard, P., and Czernik, A. J. (1999). Regulation of synaptotagmin I phosphorylation by multiple protein kinases. J. Neurochem. 73, 921-932. doi: 10.1046/j.1471-4159.1999. 0730921.x

Jenkins, M. A., Christel, C. J., Jiao, Y., Abiria, S., Kim, K. Y., Usachev, Y. M., et al. (2010). $\mathrm{Ca}^{2+}$-dependent facilitation of $\mathrm{Cav}^{1.3} \mathrm{Ca}^{2+}$ channels by densin and $\mathrm{Ca}^{2+} /$ calmodulin-dependent protein kinase II. J. Neurosci. 30, 5125-5135. doi: 10.1523/JNEUROSCI.4367-09.2010

Jiménez, J. L., and Bashir, R. (2007). In silico functional and structura characterisation of ferlin proteins by mapping disease-causing mutations and evolutionary information onto three-dimensional models of their $\mathrm{C} 2$ domains. J. Neurol. Sci. 260, 114-123. doi: 10.1016/j.jns.2007.04.016

Johnson, C. P., and Chapman, E. R. (2010). Otoferlin is a calcium sensor that directly regulates SNARE-mediated membrane fusion. J. Cell Biol. 191, 187-197. doi: $10.1083 / j \mathrm{cb} .201002089$

Jung, S., Maritzen, T., Wichmann, C., Jing, Z., Neef, A., Revelo, N. H., et al. (2015). Disruption of adaptor protein $2 \mu$ (AP- $2 \mu$ ) in cochlear hair cells impairs vesicle reloading of synaptic release sites and hearing. EMBO J. 34, 2686-2702. doi: $10.15252 /$ embj.201591885

Kantardzhieva, A. V., Peppi, M., Lane, W. S., and Sewell, W. F. (2011). Protein composition of immunoprecipitated synaptic ribbons. J. Proteome Res. 11 1163-1174. doi: $10.1021 / \mathrm{pr} 2008972$

Kazmierczak, P., Sakaguchi, H., Tokita, J., Wilson-Kubalek, E. M., Milligan, R. A Müller, U., et al. (2007). Cadherin 23 and protocadherin 15 interact to form tiplink filaments in sensory hair cells. Nature 449, 87-91. doi: 10.1038/nature06091

Kerr, A. M., Reisinger, E., and Jonas, P. (2008). Differential dependence of phasic transmitter release on synaptotagmin 1 at GABAergic and glutamatergic hippocampal synapses. Proc. Natl. Acad. Sci. U.S.A. 105, 15581-15586. doi: 10.1073/pnas.0800621105

Kim, S.-M., Kwon, M. S., Park, C. S., Choi, K.-R., Chun, J.-S., Ahn, J., et al. (2004). Modulation of Thr phosphorylation of integrin betal during muscle differentiation. J. Biol. Chem. 279, 7082-7090. doi: 10.1074/jbc.M3115 81200

Kohansal-Nodehi, M., Chua, J. J., Urlaub, H., Jahn, R., and Czernik, D. (2016) Analysis of protein phosphorylation in nerve terminal reveals extensive changes in active zone proteins upon exocytosis. elife 5:e14530. doi: 10.7554/eLife. 14530

Koos, B., Andersson, L., Clausson, C.-M., Grannas, K., Klaesson, A., Cane, G., et al. (2014). Analysis of protein interactions in situ by proximity ligation assays. Curr. Top. Microbiol. Immunol. 377, 111-126. doi: 10.1007/82_2013_334

Kujawa, S. G., and Liberman, M. C. (2009). Adding insult to injury: cochlear nerve degeneration after "temporary" noise-induced hearing loss. J. Neurosci. 29, 14077-14085. doi: 10.1523/JNEUROSCI.2845-09.2009

Lisman, J., Schulman, H., and Cline, H. (2002). The molecular basis of CaMKI function in synaptic and behavioural memory. Nat. Rev. Neurosci. 3, 175-190. doi: $10.1038 / \mathrm{nrn} 753$
Liu, X., Heidelberger, R., and Janz, R. (2014). Phosphorylation of syntaxin 3B by CaMKII regulates the formation of t-SNARE complexes. Mol. Cell. Neurosci. 60, 53-62. doi: 10.1016/j.mcn.2014.03.002

Liu, X.-B., and Murray, K. D. (2012). Neuronal excitability and calcium/calmodulin-dependent protein kinase type II: location, location, location. Epilepsia 53(Suppl. 1), 45-52. doi: 10.1111/j.1528-1167.2012. 03474.x

Llinás, R., Gruner, J. A., Sugimori, M., McGuinness, T. L., and Greengard, P. (1991) Regulation by synapsin $\mathrm{I}$ and $\mathrm{Ca}(2+)$-calmodulin-dependent protein kinase II of the transmitter release in squid giant synapse. J. Physiol. 436, 257-282. doi: 10.1113/jphysiol.1991.sp018549

Meese, S. (2015). Biochemical Studies of the Synaptic Protein Otoferlin. Ph.D. thesis University of Göttingen, Göttingen.

Merchan-Perez, A., and Liberman, M. C. (1996). Ultrastructural differences among afferent synapses on cochlear hair cells: correlations with spontaneous discharge rate. I. Comp. Neurol. 371, 208-221. doi: 10.1002/(SICI) 1096-9861(19960722) 371:2<208::AID-CNE2>3.0.CO;2-6

Meyer, A. C., Frank, T., Khimich, D., Hoch, G., Riedel, D., Chapochnikov, N. M. et al. (2009). Tuning of synapse number, structure and function in the cochlea. Nat. Neurosci. 12, 444-453. doi: 10.1038/nn.2293

Neef, J., Jung, S., Wong, A. B., Reuter, K., Pangršič, T., Chakrabarti, R., et al. (2014). Modes and regulation of endocytic membrane retrieval in mouse auditory hai cells. J. Neurosci. 34, 705-716. doi: 10.1523/JNEUROSCI.3313-13.2014

Nouvian, R., Neef, I., Bulankina, A. V., Reisinger, E., Pangršič, T., Frank, T., et al. (2011). Exocytosis at the hair cell ribbon synapse apparently operates without neuronal SNARE proteins. Nat. Neurosci. 14, 411-413. doi: 10.1038/nn.2774

Ohn, T.-L., Rutherford, M. A., Jing, Z., Jung, S., Duque-Afonso, C. J., Hoch, G., et al. (2016). Hair cells use active zones with different voltage dependence of $\mathrm{Ca}^{2+}$ influx to decompose sounds into complementary neural codes. Proc. Natl. Acad. Sci. U.S.A. 113, E4716-E4725. doi: 10.1073/pnas.1605737113

Ohyama, A., Hosaka, K., Komiya, Y., Akagawa, K., Yamauchi, E., Taniguchi, H., et al. (2002). Regulation of exocytosis through $\mathrm{Ca}^{2+} / \mathrm{ATP}$-dependent binding of autophosphorylated $\mathrm{Ca}^{2+} /$ calmodulin-activated protein kinase II to syntaxin 1A. J. Neurosci. 22, 3342-3351.

Padmanarayana, M., Hams, N., Speight, L. C., Petersson, E. J., Mehl, R. A., and Johnson, C. P. (2014). Characterization of the lipid binding properties of Otoferlin reveals specific interactions between $\mathrm{PI}(4,5) \mathrm{P} 2$ and the $\mathrm{C} 2 \mathrm{C}$ and $\mathrm{C} 2 \mathrm{~F}$ domains. Biochemistry 53, 5023-5033. doi: $10.1021 /$ bi5004469

Palmer, A. R., and Russell, I. J. (1986). Phase-locking in the cochlear nerve of the guinea-pig and its relation to the receptor potential of inner hair-cells. Hear. Res. 24, 1-15. doi: 10.1016/0378-5955(86)90002-X

Pangršič, T., Lasarow, L., Reuter, K., Takago, H., Schwander, M., Riedel, D., et al. (2010). Hearing requires otoferlin-dependent efficient replenishment of synaptic vesicles in hair cells. Nat. Neurosci. 13, 869-876. doi: 10.1038/nn.2578

Pangršič, T., Reisinger, E., and Moser, T. (2012). Otoferlin: a multi- $\mathrm{C}_{2}$ domain protein essential for hearing. Trends Neurosci. 35, 671-680. doi: 10.1016/j.tins. 2012.08.002

Pepio, A. M., and Sossin, W. S. (2001). Membrane translocation of novel protein kinase Cs is regulated by phosphorylation of the C2 domain. J. Biol. Chem. 276 3846-3855. doi: 10.1074/jbc.M006339200

Radhakrishnan, A., Stein, A., Jahn, R., and Fasshauer, D. (2009). The $\mathrm{Ca}^{2+}$ affinity of synaptotagmin 1 is markedly increased by a specific interaction of its C2B domain with phosphatidylinositol 4,5-bisphosphate. J. Biol. Chem. 284 25749-25760. doi: 10.1074/jbc.M109.042499

Ramakrishnan, N. A., Drescher, M. J., and Drescher, D. G. (2009). Direct interaction of otoferlin with syntaxin $1 \mathrm{~A}, \mathrm{SNAP}-25$, and the L-type voltagegated calcium channel Cav1.3. J. Biol. Chem. 284, 1364-1372. doi: 10.1074/jbc M803605200

Ramakrishnan, N. A., Drescher, M. J., Morley, B. J., Kelley, P. M., and Drescher D. G. (2014). Calcium regulates molecular interactions of otoferlin with SNARE proteins required for hair cell exocytosis. J. Biol. Chem. 289, 8750-8766. doi: $10.1074 /$ jbc.M113.480533

Reijntjes, D. O. J., and Pyott, S. J. (2016). The afferent signaling complex: regulation of type I spiral ganglion neuron responses in the auditory periphery. Hear. Res. 336, 1-16. doi: 10.1016/j.heares.2016.03.011

Reisinger, E., Bresee, C., Neef, J., Nair, R., Reuter, K., Bulankina, A., et al. (2011). Probing the functional equivalence of otoferlin and synaptotagmin 1 in exocytosis. J. Neurosci. 31, 4886-4895. doi: 10.1523/JNEUROSCI.5122-10.2011 
Revelo, N. H., Kamin, D., Truckenbrodt, S., Wong, A. B., Reuter-Jessen, K., Reisinger, E., et al. (2014). A new probe for super-resolution imaging of membranes elucidates trafficking pathways. J. Cell Biol. 205, 591-606. doi: 10 $1083 / \mathrm{jcb} .201402066$

Roberts, W. M. (1994). Localization of calcium signals by a mobile calcium buffer in frog saccular hair cells. J. Neurosci. 14, 3246-3262.

Romani, A., and Scarpa, A. (1992). Regulation of cell magnesium. Arch. Biochem Biophys. 298, 1-12. doi: 10.1016/0003-9861(92)90086-C

Roux, I., Hosie, S., Johnson, S. L., Bahloul, A., Cayet, N., Nouaille, S., et al. (2009). Myosin VI is required for the proper maturation and function of inner hair cell ribbon synapses. Hum. Mol. Genet. 18, 4615-4628. doi: 10.1093/hmg/ ddp 429

Roux, I., Safieddine, S., Nouvian, R., Grati, M., Simmler, M.-C., Bahloul, A., et al (2006). Otoferlin, defective in a human deafness form, is essential for exocytosis at the auditory ribbon synapse. Cell 127, 277-289. doi: 10.1016/j.cell.2006. 08.040

Rutherford, M. A., Chapochnikov, N. M., and Moser, T. (2012). Spike encoding of neurotransmitter release timing by spiral ganglion neurons of the cochlea. J. Neurosci. 32, 4773-4789. doi: 10.1523/JNEUROSCI.4511 11.2012

Sadanandappa, M. K., Blanco Redondo, B., Michels, B., Rodrigues, V., Gerber, B. VijayRaghavan, K., et al. (2013). Synapsin function in GABA-ergic interneurons is required for short-term olfactory habituation. J. Neurosci. 33, 16576-16585. doi: 10.1523/JNEUROSCI.3142-13.2013

Sahdev, S., Khattar, S. K., and Saini, K. S. (2008). Production of active eukaryotic proteins through bacterial expression systems: a review of the existing biotechnology strategies. Mol. Cell. Biochem. 307, 249-264. doi: 10.1007/ s11010-007-9603-6

Shao, X., Fernandez, I., Südhof, T. C., and Rizo, J. (1998). Solution structures of the $\mathrm{Ca}^{2+}$-free and $\mathrm{Ca}^{2+}$-bound $\mathrm{C}_{2} \mathrm{~A}$ domain of synaptotagmin I: does $\mathrm{Ca}^{2+}$ induce a conformational change? Biochemistry 37, 16106-16115.

Shevchenko, A., Tomas, H., Havlis, J., Olsen, J. V., and Mann, M. (2006). In-ge digestion for mass spectrometric characterization of proteins and proteomes. Nat. Protoc. 1, 2856-2860. doi: 10.1038/nprot.2006.468

Stokoe, D., Caudwell, B., Cohen, P. T., and Cohen, P. (1993). The substrate specificity and structure of mitogen-activated protein (MAP) kinaseactivated protein kinase-2. Biochem. J. 296(Pt 3), 843-849. doi: 10.1042/bj29 60843

Strenzke, N., Chakrabarti, R., Al-Moyed, H., Müller, A., Hoch, G., Pangršič, T., et al (2016). Hair cell synaptic dysfunction, auditory fatigue and thermal sensitivity in otoferlin Ile515Thr mutants. EMBO J. 35, 2519-2535. doi: 10.15252/embj 201694564

Studier, F. W. (2005). Protein production by auto-induction in high density shaking cultures. Protein Expr. Purif. 41, 207-234. doi: 10.1016/j.pep.2005. 01.016

Sumi, M., Kiuchi, K., Ishikawa, T., Ishii, A., Hagiwara, M., Nagatsu, T. et al. (1991). The newly synthesized selective $\mathrm{Ca}^{2+} /$ calmodulin dependent protein kinase II inhibitor $\mathrm{KN}-93$ reduces dopamine contents in $\mathrm{PC} 12 \mathrm{~h}$ cells.
Biochem. Biophys. Res. Commun. 181, 968-975. doi: 10.1016/0006-291X(91) 92031-E

Taberner, A. M., and Liberman, M. C. (2005). Response properties of single auditory nerve fibers in the mouse. J. Neurophysiol. 93, 557-569. doi: 10.1152/ jn.00574.2004

Ubach, J., Zhang, X., Shao, X., Südhof, T. C., and Rizo, J. (1998). Ca ${ }^{2+}$ binding to synaptotagmin: how many $\mathrm{Ca}^{2+}$ ions bind to the tip of a $\mathrm{C}_{2}$-domain? EMBO J 17, 3921-3930. doi: 10.1093/emboj/17.14.3921

Uthaiah, R. C., and Hudspeth, A. J. (2010). Molecular anatomy of the hair cell's ribbon synapse. J. Neurosci. 30, 12387-12399. doi: 10.1523/JNEUROSCI.101410.2010

van den Bogaart, G., Meyenberg, K., Diederichsen, U., and Jahn, R. (2012). Phosphatidylinositol 4,5-bisphosphate increases the $\mathrm{Ca}^{2+}$ affinity of synaptotagmin-1 40-fold. J. Biol. Chem. 287, 16447-16453. doi: $10.1074 / j b c . M 112.343418$

Washington, N. L., and Ward, S. (2006). FER-1 regulates $\mathrm{Ca}^{2+}$-mediated membrane fusion during C. elegans spermatogenesis. J. Cell. Sci. 119, 25522562. doi: $10.1242 /$ jcs. 02980

White, R. R., Kwon, Y. G., Taing, M., Lawrence, D. S., and Edelman, A. M. (1998). Definition of optimal substrate recognition motifs of $\mathrm{Ca}^{2+}$-calmodulindependent protein kinases IV and II reveals shared and distinctive features. J. Biol. Chem. 273, 3166-3172. doi: 10.1074/jbc.273.6.3166

Wong, A. B., Rutherford, M. A., Gabrielaitis, M., Pangršič, T., Göttfert, F., Frank, T., et al. (2014). Developmental refinement of hair cell synapses tightens the coupling of $\mathrm{Ca}^{2+}$ influx to exocytosis. EMBO J. 33, 247-264. doi: $10.1002 /$ embj. 201387110

Yasunaga, S., Grati, M., Cohen-Salmon, M., El-Amraouii, A., Mustapha, M., Salem, N., et al. (1999). A mutation in OTOF, encoding otoferlin, a FER-1like protein, causes DFNB9, a nonsyndromic form of deafness. Nat. Genet. 21 , 363-369. doi: $10.1038 / 7693$

Yu, H., Pan, B., Weyer, A., Wu, H.-E., Meng, J., Fischer, G., et al. (2015). CaMKII controls whether touch is painful. J. Neurosci. 35, 14086-14102. doi: 10.1523/ JNEUROSCI.1969-15.2015

Zak, M., Breß, A., Brandt, N., Franz, C., Ruth, P., Pfister, M., et al. (2012). Ergic2, a brain specific interacting partner of otoferlin. Cell. Physiol. Biochem. 29 941-948. doi: 10.1159/000188338

Conflict of Interest Statement: The authors declare that the research was conducted in the absence of any commercial or financial relationships that could be construed as a potential conflict of interest.

Copyright (c) 2017 Meese, Cepeda, Gahlen, Adams, Ficner, Ricci, Heller, Reisinge and Herget. This is an open-access article distributed under the terms of the Creative Commons Attribution License (CC BY). The use, distribution or reproduction in other forums is permitted, provided the original author(s) or licensor are credited and that the original publication in this journal is cited, in accordance with accepted academic practice. No use, distribution or reproduction is permitted which does not comply with these terms. 


\section{Statistical analysis}

Appendix Supplementary Table 1. Mean averages, sample size and statistical analysis.

Data information: s.e.m., standard error of the mean; N, number of animals; n, number of cells.

Figure 3.9

\begin{tabular}{|l|c|c|c|c|}
\hline \multirow{2}{*}{ Genotype/Condition } & \multicolumn{2}{|c|}{ Mean \pm s.e.m. } & \multirow{2}{*}{ N } & \multirow{2}{*}{} \\
\cline { 2 - 4 } & Parvalbumin & Otoferlin & 197 & 8 \\
\hline WT & $100 \pm 1 \%$ & $100 \pm 1 \%$ & 61 & 2 \\
\hline Otof $^{\text {I515T/I515T }}$ & $79 \pm 2 \%$ & $48 \pm 3 \%$ & 127 & 3 \\
\hline Otof $^{+-}$ & $68 \pm 2 \%$ & $53 \pm 1 \%$ & 74 & 4 \\
\hline Otof $^{-/-}$ & $54 \pm 4 \%$ & $8 \pm 1 \%$ & & 4 \\
\hline
\end{tabular}

\begin{tabular}{|c|c|c|c|}
\hline Compared group & $\begin{array}{c}\text { Statistical } \\
\text { significance }\end{array}$ & $P$-value & Statistical test \\
\hline \multicolumn{4}{|l|}{ Parvalbumin levels: } \\
\hline WT vs. Otof ${ }^{1515 T / 1515 T}$ & $* * *$ & $<0.0001$ & \multirow{6}{*}{$\begin{array}{l}\text { Kruskal-Wallis test followed by } \\
\text { Dunn's multiple comparison test }\end{array}$} \\
\hline WT vs. Otof ${ }^{+/}$ & $* * *$ & $<0.0001$ & \\
\hline WT vs. Otof ${ }^{-/-}$ & $* * *$ & $<0.0001$ & \\
\hline Otof ${ }^{1515 T / 1515 T}$ vs. Otof ${ }^{+/-}$ & ns & 0.3296 & \\
\hline Otof ${ }^{I 515 T / 1515 T}$ vs. Otof ${ }^{-/-}$ & ** & 0.0015 & \\
\hline Otof $^{+/-}$vs. Otof ${ }^{-/-}$ & ns & 0.3481 & \\
\hline \multicolumn{4}{|l|}{ Otoferlin levels: } \\
\hline WT vs. Otof ${ }^{1515 T / 1515 T}$ & $* * *$ & $<0.0001$ & \multirow{6}{*}{$\begin{array}{l}\text { Kruskal-Wallis test followed by } \\
\text { Dunn's multiple comparison test }\end{array}$} \\
\hline WT vs. Otof ${ }^{+/-}$ & $* * *$ & $<0.0001$ & \\
\hline WT vs. Otof ${ }^{-/-}$ & $* * *$ & $<0.0001$ & \\
\hline Otof ${ }^{I 515 T / 1515 T}$ vs. Otof ${ }^{-/-}$ & $\mathrm{ns}$ & $>0.9999$ & \\
\hline Otof ${ }^{\text {I515T/I515T }}$ vs. Otof ${ }^{-/-}$ & $* * *$ & 0.0001 & \\
\hline Otof $^{+/-}$vs. Otof ${ }^{-/-}$ & $* * *$ & $<0.0001$ & \\
\hline
\end{tabular}

Figure 3.10

\begin{tabular}{|l|c|c|c|c|}
\hline \multirow{2}{*}{ Genotype/Condition } & \multicolumn{2}{|c|}{ Mean \pm s.e.m. } & \multirow{2}{*}{ N } & \multirow{2}{*}{ N } \\
\cline { 2 - 3 } & Calretinin & Otoferlin & & \\
\hline WT & $100 \pm 1 \%$ & $100 \pm 1 \%$ & 98 & 4 \\
\hline Otof IIITTIIIIST $^{\text {Otof }}{ }^{-/}$ & $97 \pm 4 \%$ & $48 \pm 1 \%$ & 74 & 2 \\
\hline
\end{tabular}

\begin{tabular}{|c|c|c|c|}
\hline Compared group & $\begin{array}{l}\text { Statistical } \\
\text { significance }\end{array}$ & $P$-value & Statistical test \\
\hline \multicolumn{4}{|l|}{ Calretinin levels: } \\
\hline WT vs. Otof f515T/III5T $^{2}$ & ns & $>0.9999$ & \multirow{3}{*}{$\begin{array}{l}\text { Kruskal-Wallis test followed by } \\
\text { Dunn's multiple comparison test }\end{array}$} \\
\hline WT vs. Otof $f^{-/-}$ & $* * *$ & $<0.0001$ & \\
\hline Otof ${ }^{\text {I515T/I515T vs. Otof }}{ }^{-/-}$ & $* * *$ & $<0.0001$ & \\
\hline \multicolumn{4}{|l|}{ Otoferlin levels: } \\
\hline WT vs. Otof ${ }^{\text {I515T/I515T }}$ & $* * *$ & $<0.0001$ & \multirow{3}{*}{$\begin{array}{l}\text { Kruskal-Wallis test followed by } \\
\text { Dunn's multiple comparison test }\end{array}$} \\
\hline WT vs. Otof $f^{-/-}$ & *** & $<0.0001$ & \\
\hline Otof ${ }^{1515 T / 1515 T}$ vs. Otof ${ }^{-/-}$ & ** & 0.0030 & \\
\hline
\end{tabular}




\section{Acknowledgments}

First and foremost, I thank PD Dr. Ellen Reisinger for her supervision, guidance, and for giving me the opportunity to work in her team on this interesting topic.

I would especially like to thank Prof. Dr. Silvio Rizzoli and Prof. Dr. Manuela Schmidt for being members of my Ph.D. thesis committee, for the fruitful discussions and ideas.

I would also like to thank the University Medical Center Göttingen, and the Deutsche Forschungsgemeinschaft (DFG) through the SFB 889 Collaborative Research Center "Cellular Mechanisms of Sensory Processing” and the Göttingen Graduate Center for Neurosciences, Biophysics, and Molecular Biosciences (GGNB), for the financial support.

I want to thank my colleagues at the InnerEarLab for their help and support throughout my studies. Special thanks go to Nina-Katrin Dankenbrink-Werder for daily assistance in the lab and considerable genotyping work; Gerhard Hoch for developing the Matlab/Imaris analysis routine used in this work; Dennis Spormann and Dominik Riemenschneider for the IT support.

I am very grateful to all my collaborators on projects contributing to this thesis as well as to projects not included within this thesis. I thank Dr. Meike Herget and Dr. Sandra Meese for the pioneer work on the CaMKII project. Dr. Christof Lenz and Lisa Neuenroth for the excellent mass spectrometry work and contribution to the PKC project. To Prof. Dr. Reinhard Jahn and Prof. Dr. Henning Urlaub for fruitful discussions and guidance.

I wish to extend my most sincere gratitude to Dr. Momchil Ninov for his dedication in helping me establish new experimental routines, for the exciting scientific discussions, for the constant support, for always being able to lift up my spirit, and for his friendship.

I would like to thank Dr. John Murdoch for the countless proofreading sessions and English corrections for the thesis and publications, and for his friendship.

I am deeply grateful to my colleague Dr. Hanan Al-Moyed for her contribution to the PKC project, her continuous support throughout my thesis in many respects, and especially for her friendship. "When the snows fall and the white winds blow, the lone wolf dies, but the pack survives.".

Last but not least, I thank my family and friends for their constant support and encouragement throughout my Ph.D. studies. Aos meus pais e irmã, pelo apoio incondicional, força e incentivo ao longo destes anos. Pelas inúmeras encomendas do "fiel amigo", azeite, vinho do Porto, chouriços, queijos, etc. que me permitiram manter um cantinho do nosso Portugal sempre comigo. Um obrigada especial à Rita e à Filipa, que apesar da distância sempre estiveram presentes. 
